\title{
Simulations of magnetoconvection in cool main-sequence stars
}

\author{
Dissertation \\ zur Erlangung des mathematisch-naturwissenschaftlichen Doktorgrades \\ "Doctor rerum naturalium" \\ der Georg-August-Universität Göttingen \\ im Promotionsprogramm PROPHYS \\ der Georg-August University School of Science (GAUSS)
}

\author{
vorgelegt von \\ Benjamin Beeck
}

aus Eutin

Göttingen, 2014 
Betreuungsausschuss

Prof. Dr. Manfred Schüssler

Max-Planck-Institut für Sonnensystemforschung, Göttingen, Germany

Prof. Dr. Ansgar Reiners

Institut für Astrophysik, Georg-August-Universität Göttingen, Germany

Prof. Dr. Stefan Dreizler

Institut für Astrophysik, Georg-August-Universität Göttingen, Germany

Mitglieder der Prüfungskommision

Referent: Prof. Dr. Ansgar Reiners

Institut für Astrophysik, Georg-August-Universität Göttingen, Germany

Korreferent: Prof. Dr. Manfred Schüssler

Max-Planck-Institut für Sonnensystemforschung, Göttingen, Germany

2. Korreferent: Prof. Dr. Robert F. Stein

Physics and Astronomy Department, Michigan State University, East Lansing, MI, USA

Weitere Mitglieder der Prüfungskommission:

Prof. Dr. Stefan Dreizler

Institut für Astrophysik, Georg-August-Universität Göttingen, Germany

Prof. Dr. Hardi Peter

Max-Planck-Institut für Sonnensystemforschung, Göttingen, Germany

Prof. Dr. Wolfgang Glatzel

Institut für Astrophysik, Georg-August-Universität Göttingen, Germany

Prof. Dr. Wolfram Kollatschny

Institut für Astrophysik, Georg-August-Universität Göttingen, Germany

Tag der mündlichen Prüfung: 14. Februar 2014 


\section{Bibliografische Information der Deutschen Nationalbibliothek}

Die Deutsche Nationalbibliothek verzeichnet diese Publikation in der Deutschen Nationalbibliografie; detaillierte bibliografische Daten sind im Internet über http: //dnb . d-nb . de abrufbar.

ISBN 978-3-944072-00-5

uni-edition $\mathrm{GmbH} 2014$

http://www.uni-edition.de

(C) Benjamin Beeck

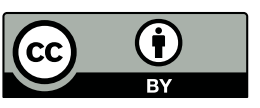

This work is distributed under a

Creative Commons Attribution 3.0 License

Printed in Germany 
Cover figure: Illustration of some physical quantities in the simulation box of the M2V star with an average vertical magnetic field of $B_{0}=500 \mathrm{G}$. The silvery surface reperesents the optical surface, where $\tau_{\mathrm{R}}=1$; the red tubes indicate magnetic field lines; the bottom face of the box shows the vertical velocity near the bottom of the box (blue $=$ upflows, red = downflows); the side faces show the temperature fluctuations (blue = cooler than horizontal mean; red $=$ hotter than horizontal mean). The physical dimensions of the simulaton box are $(X, Y, Z)=(1.56 \mathrm{Mm}, 1.56 \mathrm{Mm}, 0.8 \mathrm{Mm})$ resolved in $512 \times 512 \times$ 250 cells. 


\section{Contents}

$\begin{array}{ll}\text { Summary } & 7\end{array}$

1 Introduction 9

2 Numerical codes and simulation setup $\quad 13$

2.1 The MURaM code . . . . . . . . . . . . . . . . . . . . 13

2.1.1 Compressible magnetohydrodynamics . . . . . . . . . . . 13

2.1.2 The numerical scheme . . . . . . . . . . . . . . . 14

2.1.3 Treatment of radiation . . . . . . . . . . . . . 15

2.2 The SPINOR code . . . . . . . . . . . . . . . . . . . . . . . . . 17

2.3 Simulation setup . . . . . . . . . . . . . . . 17

2.3.1 Stellar parameters . . . . . . . . . . . . . . 17

2.3.2 Setup of the non-magnetic simulations . . . . . . . . . . 18

2.3.3 Setup of the magnetic simulations . . . . . . . . . . . 19

3 Overall structure 23

3.1 Overall structure of the non-magnetic simulations . . . . . . . . . . 23

3.1.1 General morphology of near-surface convection . . . . . . . . . 23

3.1 .2 Velocity field ......................... 24

3.1.3 Temperature, pressure, and density ............ 30

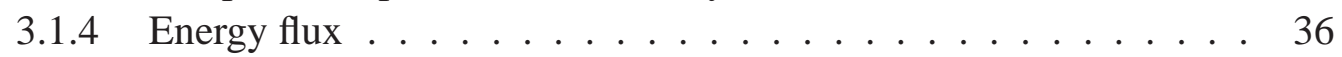

3.1.5 Comparison to 1D models . . . . . . . . . . . . . 37

3.2 Structure of the magnetic field . . . . . . . . . . . . . . . 40

3.3 Effects of the magnetic field on the atmospheric structure . . . . . . . . 52

3.3.1 Optical depth structure . . . . . . . . . . . . . . 52

3.3 .2 Velocity field . . . . . . . . . . . . . . . . 57

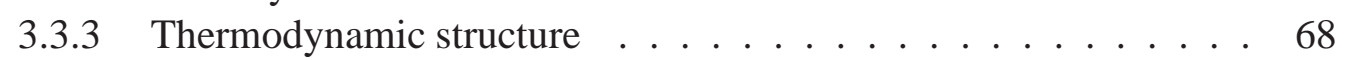

3.3 .4 Energy flux ...................... 78

4 Granulation $\quad \mathbf{8 1}$

4.1 Granulation segmentation . . . . . . . . . . . . . . . . . . 81

4.2 Granulation in non-magnetic runs . . . . . . . . . . . . . . . 84

4.2.1 General appearance of the granulation pattern . . . . . . . . . . 84

4.2 .2 Vortex motions . . . . . . . . . . . . . . . 86

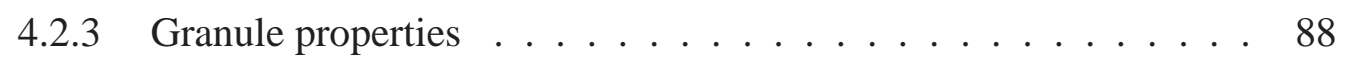

4.2 .4 Granule lifetime . . . . . . . . . . . . . . . . . . . 94 
4.3 Effects of the magnetic field on the granulation . . . . . . . . . . 97

4.3.1 Vertically emerging intensity . . . . . . . . . . . . . 97

4.3.2 Granule properties . . . . . . . . . . . . . 100

4.3.3 Temporal evolution of individual magnetic features . . . . . . . 102

$5 \quad$ Limb darkening 105

5.1 Centre-to-limb variation of the intensity without magnetic field . . . . . . 105

5.2 Effect of the magnetic field . . . . . . . . . . . . . . 108

6 Synthetic spectral lines $\mathbf{1 1 5}$

6.1 Line synthesis and stellar disc integration . . . . . . . . . . . . . 115

6.2 Spectral lines without magnetic fields . . . . . . . . . . . . 117

6.2.1 Spatially resolved line profiles . . . . . . . . . . . . . 117

6.2.2 Centre-to-limb variation of line profiles . . . . . . . . . . . . 117

6.2 .3 Disc-integrated line profiles . . . . . . . . . . . . . 121

6.2.4 Comparison to observational data . . . . . . . . . . 123

6.3 Effects of the magnetic fields . . . . . . . . . . . . . . . . . 124

6.3 .1 Spatially resolved spectra . . . . . . . . . . . 126

6.3.2 Centre-to-limb variation of spectral line profiles . . . . . . . . . . 132

6.3 .3 Disc-integrated spectra . . . . . . . . . . . . . . 137

6.3.4 Zeeman effect vs. thermodynamic effects . . . . . . . . . . . . . 142

6.4 Test case: two-component measurement of the magnetic field . . . . . . 144

7 Conclusion 149

7.1 Brief summary of the results . . . . . . . . . . . . . . . . . . . 149

7.2 Relevance for stellar physics . . . . . . . . . . . . . . . 150

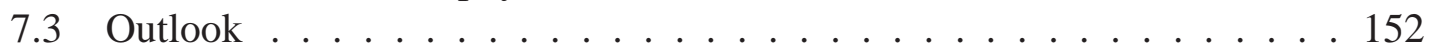

$\begin{array}{ll}\text { Bibliography } & 155\end{array}$

$\begin{array}{ll}\text { A Horizontal averages } & 165\end{array}$

$\begin{array}{llr}\text { B Additional figures } & 169\end{array}$

$\begin{array}{ll}\text { Publications } & 197\end{array}$

$\begin{array}{ll}\text { Acknowledgements } & 199\end{array}$

$\begin{array}{ll}\text { Lebenslauf } & 201\end{array}$ 


\section{Summary}

In this thesis, the magnetoconvective processes in the near-surface layers of cool mainsequence stars were studied in 24 three-dimensional local "box-in-a-star" simulations. For each of six sets of stellar parameters corresponding to spectral types F3V-M2V including the solar case $(\mathrm{G} 2 \mathrm{~V})$, one non-magnetic and three magnetic simulations with an initially vertical and homogeneous field of 20,100, and $500 \mathrm{G}$ were performed.

Analogous to the solar example, hot upwellings (granules) and a network of cool downflows evolve. In the magnetic case, the magnetic flux is advected by the granular outflows and concentrated in the intergranular lane network, where it causes local depressions in the optical surface and impedes the convective flows. This leads to the formation of small bright and larger dark structures. In the upper photospheres, the magnetic field considerably modifies the non-radiative heating processes in all simulated stars.

A granule segmentation and tracking algorithm was developed and the granulation was analysed for the non-magnetic and some magnetic runs. The sizes and lifetimes of granules agree with observations in the solar case. A marginal influence of the magnetic field on the statistical properties of granules was found.

For a few wavelength passbands, the centre-to-limb variation of the intensity and its rms contrast were calculated on the basis of snapshots from the simulations. The limb darkening in the non-magnetic case agrees relatively well with the limb darkening derived from 1D atmospheres. The deviations of the different magnetic cases from the nonmagnetic case are large due to a brightening of the limb by magnetic flux concentrations appearing as "faculae".

Synthetic spectral line profiles were calculated for the simulated stars. A disc-integration including differential rotation was carried out in order to study the effects of the three-dimensional atmospheric structure on spectral line profiles. A dependence of the line bisector shapes on differential rotation parameter and inclination was found. The impact of the magnetic field on the Stokes I component of some Zeeman-sensitive lines was analysed. While in M-type stars the Zeeman effect substantially broadens the lines for an average surface field strength of $500 \mathrm{G}$, in hotter stars the modified atmospheric structure (due to the field) has a strong additional impact on the line profiles, e.g. line weakening due to ionisation. This can considerably impair magnetic field measurements as it usually leads to narrower line profiles. Moreover, the correlation between magnetic field and velocity field leads to a net Doppler shift of spectral lines.

Although the simulations presented in this thesis only cover parts of the phenomena of stellar surface magnetism (excluding, e. g., starspots and bipolar active regions), they are an essential step towards a physically comprehensive description of magnetoconvective processes in stars, which is needed, e. g. for the improvement of inversion methods and the correct interpretation of spectroscopic observations. 



\section{Introduction}

Stellar magnetic fields are the driver of a number of phenomena, acting on different time and length scales and in different phases of stellar evolution. For example, magnetic instabilities play a role in the formation processes of stars and their planetary systems (Hennebelle and Teyssier 2008, Johansen 2009). During the early phase of the mainsequence evolution, the magnetic field is responsible for a significant loss of angular momentum (Reiners and Mohanty 2012). Evolved stellar objects such as white dwarfs can have strong surface magnetism (e.g. Kuelebi et al. 2010) and it has been suggested that, in cases of extreme magnetism, the structure of these objects is crucially changed, so that the Chandrasekhar mass limit maybe violated (cf. Das and Mukhopadhyay 2012, Chamel et al. 2013).

In the Sun, the magnetic field causes dark spots, bright faculae, and chromospheric and coronal UV and X-ray emission (see Schrijver and Zwaan 2000, for a review). These and various other phenomena are referred to as stellar/solar (magnetic) activity. The level of the solar activity varies in an 11-year cycle (for a review see Hathaway 2010). The solar activity cycle influences not only the solar surface but also has considerable impact on the entire heliosphere, including the Earth. For instance, the solar activity leads to coronal mass ejections and geomagnetic storms (Chen 2011), which can cause considerable problems for human high-tech activities (Schwenn 2006, Pulkkinen 2007). Moreover, the total and spectral irradiance of the Sun vary with the solar cycle (Ball et al. 2012, Thuillier et al. 2013). Consequently, the solar activity probably plays some albeit small role in global climate variations (cf. Solanki et al. 2013).

The very dynamic magnetic field at the surface of the Sun is shaped by its interaction with convective flows. Cool main-sequence stars such as the Sun have thick convective envelopes (for stellar masses $M \gtrsim 0.3 M_{\odot}$ ) or are fully convective (if $M \lesssim 0.3 M_{\odot}$ ). The magnetic field is amplified, restructured, and transported by convective motions (for reviews, see Schrijver and Zwaan 2000, Fan 2009, Stein 2012, Schüssler 2013). In the Sun, there is evidence for a global flux-transport dynamo, which is mainly responsible for the emergence of magnetic flux in active regions and thus shapes the 11-year activity cycle (e.g. Rempel 2006). In addition, small-scale dynamo action has been proposed for the near-surface layers (see Pietarila Graham et al. 2010, and references therein).

Magnetic field measurements of spatially resolved magnetic features in the solar photosphere exploit the polarisation, broadening, and splitting of (some) spectral lines in the presence of a magnetic field owing to the Zeeman effect. For resolved magnetic structures, the circularly polarised light (Stokes V) is a very sensitive measure of the (line-of-sight component of the) magnetic field. The measurements reveal a very inhomogeneous magnetic field on the Sun's surface: in sunspot umbrae, the field strength at the optical surface can locally reach $2-3 \mathrm{kG}$, while most of the visible surface is nearly field-free (e.g. Schri- 
jver and Zwaan 2000). Complementary measurements using the Hanle effect indicate a volume-filling magnetic field of about $130 \mathrm{G}$ in the photosphere with polarities mixed on unresolved scales; consequently, its Zeeman-effect signal in the circular polarisation is canceled (Trujillo Bueno et al. 2004).

For the Sun, there exist strong temporal correlations between different phenomena caused by the magnetic field such as sunspot number, X-ray flux, chromospheric H- $\alpha$ emission, etc. (see, e. g. Bachmann et al. 2004, Orlando et al. 2004). For stars other than the Sun, it is much more difficult to use the Zeeman and Hanle effects to measure their surface magnetic field, which is invariably not fully resolved. Consequently, information about the magnetic activity of stars is usually obtained by measurements of activity indicators such as fluxes from chromospheric emission lines ( $\mathrm{H} \alpha, \mathrm{Ca}$ II, etc.) or X-rays. According to these activity indicators, especially young stars and very-low-mass stars, are far more magnetically active than the Sun, while the solar activity level is typical for solar-like stars of an age of several billion years (Güdel 2007, Reiners 2012). The high photometric and spectroscopic variability of very active stars has a negative impact on, e. g., the detectability of planetary companions (cf. Jeffers et al. 2013, Cegla et al. 2013). Moreover, due to the high UV fluxes and variability linked to stellar activity, it may also play an important role in the habitability of planets.

For some stars, especially cool stars of spectral types K and M, there have been measurements of the magnetic field using the Zeeman and Hanle effects. Some active M stars were found to have global average magnetic fields of up to several $\mathrm{kG}$ (Reiners and Basri 2007). With Zeeman-Doppler imaging (Semel 1989, ZDI), maps of the magnetic field could be obtained for some active stars from time-resolved spectropolarimetric data. This method attempts to invert the combined Doppler and Zeeman effects on the Stokes I and $\mathrm{V}$ parameters ${ }^{1}$ of spectral lines. The resulting maps often show large polar "spots" with strong azimuthal fields (Donati et al. 2003, Petit et al. 2004).

The magnetic field measurement of stars are often made neglecting the unknown effects of the magnetic field on the atmospheric structure. If the magnetic field is very inhomogeneously distributed and has a strong effect on the convection, it can be expected that the atmospheric structure is not independent of the field strength but that temperature, velocities, gas pressure, etc. statistically depend the local direction and strength of the magnetic field. Consequently, the magnetic component of the stellar surface (e.g. starspots) has very different properties from the non-magnetic component. If these differences are neglected, the measurement of the magnetic field could involve large systematic errors (cf. Rosén and Kochukhov 2012). A detailed knowledge of the impact of the magnetic field on the local atmospheric structure of stars of different parameters is therefore essential to verify or improve the results obtained by stellar magnetic field measurements.

The knowledge and understanding of the physical background of magnetic phenomena in the solar photosphere have seen much progress in recent years. On the one hand, new observational data from space (e. g. Hinode, SDO) and balloon-borne missions (Sunrise) have produced a huge amount of observational data with unprecedented quality. On the other hand, the theory of the solar near-surface magnetoconvection has flourished owing to the growing computational power, which facilitates comprehensive 3D radiative MHD simulations. The first fully compressible simulation of the solar surface magneto-

\footnotetext{
${ }^{1}$ Ideally, ZDI also uses the Stokes U and Q components; the SNR of these are, however, generally much too low.
} 
convection have been run by Nordlund and Stein (1990) illustrating the modified convective flows in a small surface region (few granules) with $500 \mathrm{G}$ average field. More recent simulations of the solar surface magneto-convection cover many different phenomena and scales comprising the small-scale dynamo in intergranular lanes (Pietarila Graham et al. 2010), umbral convection (Schüssler and Vögler 2006, Bharti et al. 2010), solar pores (Cameron et al. 2007), sunspots (Rempel et al. 2009), and whole active regions (Cheung et al. 2010, Stein et al. 2011). Reviews on the results of solar surface magnetoconvection are given by, e.g., Stein (2012) and Schüssler (2013). Most relevant in the context of this thesis are simulations of unipolar weak to intermediate strength magnetised regions (plage regions) as previously investigated by, e. g., Nordlund and Stein (1990), Stein et al. (2002), Vögler (2003), Vögler et al. (2005), and Moll et al. (2012).

The comparison of the results obtained from such comprehensive simulations with spatially well-resolved observations show an excellent agreement, most of the deviations investigated thus for being caused by instrumental effects in the observations rather than by systematic errors in the simulations (e. g. Keller et al. 2004, Cheung et al. 2008, Bello González et al. 2009, Beeck et al. 2013b). Beeck et al. (2012) carried out a comparison of three different numerical codes to cross-validate the results obtained with them.

For stars other than the Sun, a number of comprehensive hydrodynamic simulations (without magnetic field) have been conducted in recent years to address various scientific questions. Following the pioneering work of Nordlund and Dravins (1990a), who simulated four stars with parameters roughly resembling those of Procyon, $\alpha$ Cen A, $\alpha$ Cen $B$, and $\beta H y i$, the simulations were extended to less solar-like objects such as white dwarfs (Ludwig et al. 1994), M-type main-sequence and pre-main-sequence stars (Ludwig et al. 2002, 2006), red giants (Collet et al. 2007), and metal-poor stars (Collet et al. 2011). Recently, grids of hydrodynamical models have been developed for the purpose of fitting spectroscopic observations with synthetic spectra generated from the simulation results and thus determining the stellar parameters(Trampedach et al. 2013, Magic et al. 2013). Although the range of parameters spanned by the different stellar types is large, the general picture of asymmetric convection with slowly expanding upflows and a network of entropy deficient, dense downflows is qualitatively similar in all these cases. Quantitatively, the flow characteristics vary strongly with spectral type, which promotes the necessity of 3D simulations to correctly interpret stellar spectra.

Simulations and observations of the Sun indicate that the magnetic field plays a key role in the physical interpretation of many observable phenomena in the solar atmosphere. Therefore, it is an important task to include the magnetic field in simulations for other stars, which generally have similar or much stronger surface magnetism compared to the Sun. A detailed understanding of the physical processes in stellar atmospheres with a magnetic field is also an essential step to improve magnetic field measurements in stars on the basis of spectroscopic and spectropolarimetric data. The first radiative MHD simulations of stellar atmospheric layers were presented by Beeck et al. (2011). Wedemeyer et al. (2013) recently published MHD simulations of the chromospheres of M-type dwarfs.

In this thesis, radiative (M)HD simulations of main-sequence stars of six different sets of stellar parameters are analysed. For each of the six simulated stars there is one nonmagnetic and three magnetic simulations with different field strengths (signed average vertical field strength of 20,100, and $500 \mathrm{G}$, respectively). The sections describing the non-magnetic simulations (Sects. 3.1, 4.1, 4.2, 5.1, 6.1, 6.2) have been published in Beeck 
et al. (2013a) and Beeck et al. (2013b). ${ }^{2}$

The thesis is structured as follows: Sect. 2 briefly introduces the applied numerical codes and explains the setup of the simulations. Sect. 3 describes the overall properties of the simulation results. In Sect. 4 the granulation pattern and the vertically emerging intensity are analysed. Sect. 5 details the centre-to-limb variation of the intensity and its rms contrast, while in Sect. 6 synthetic spectral line profiles are analysed. A concluding section, Sect. 7, with a brief summary of the results, implications for stellar physics, and an outlook closes the thesis.

\footnotetext{
${ }^{2} \mathrm{~A}$ footnote to the title of each already published section gives the reference to the corresponding paper.
} 


\section{Numerical codes and simulation setup}

The results presented in Sect. 3 - 6 are based on two numerical codes: the magnetohydrodynamics (MHD) code MURaM and the spectral line synthesis code SPINOR. In this section, the relevant physical background and the numerical methods of both codes are briefly delineated and the simulation setup is given. For a more detailed description of the codes themselves, see Vögler (2003) and Vögler et al. (2005) for the MURaM code and Frutiger (2000) and Frutiger et al. (2000) for the SPINOR code.

\subsection{The MURaM code}

\subsubsection{Compressible magnetohydrodynamics}

The dynamics of plasmas (and electrically conducting fluids in general) are much more complicated than the dynamics of uncharged isolating fluids because of the long-range interaction between particles through electromagnetic fields. Magnetohydrodynamics (MHD) is a powerful way to describe the physics in a well-conducting fluid under certain conditions. The key prerequisites of MHD are that no charge separation takes place on resolved scales and that all phase velocities are much smaller than the speed of light. Consequently, the displacement current can be dropped from the Maxwell equations. These assumptions hold when all time and length scales considered are large compared to the inherent scales of the plasma, such as the Debye length $\lambda_{D}$, the inverse of the plasma frequency, $\omega_{p}^{-1}$, and the gyro-radii of the charged particles. Fortunately, these scales are very small in stellar atmospheres ${ }^{1}$ while the spatial resolution of the simulations considered here are of the order of several $\mathrm{km}$ and the temporal resolution is $\sim 0.1 \mathrm{~s}$. This implies that MHD can be used to describe the relevant dynamics of stellar atmospheres.

In what follows, $\varrho=\varrho(x, y, z, t), \mathbf{v}=\mathbf{v}(x, y, z, t), p=p(x, y, z, t), \mathbf{B}=\mathbf{B}(x, y, z . t)$, and $e=e(x, y, z, t)$ are the fields of mass density, velocity, pressure, the magnetic field, and the internal energy (per volume), respectively, in three-dimensional space with time, $t$. In

\footnotetext{
${ }^{1}$ With realistic upper and lower limits for the electron density, $n_{e}$, the temperature, $T$, and the modulus of the magnetic field, $B$, the following upper limits for the three mentioned inherent scales follow: gyro-radius of a proton with $B>0.01 \mathrm{G}$ and $v<30 \mathrm{~km} \mathrm{~s}^{-1}: r_{p}<10^{-7} \mathrm{~cm}$; inverse of plasma frequency with with $n_{e}>10^{6} \mathrm{~cm}^{-3}: \omega_{p}^{-1}<2 \cdot 10^{-8} \mathrm{~s}$; Debye length with $n_{e}>10^{6} \mathrm{~cm}^{-3}$ and $T>2500 \mathrm{~K}: \lambda_{D}<0.25 \mathrm{~cm}$. The low ionisation degree and collisions with neutrals in very-cool-star atmospheres can, however, substantially increase the gyro-radius. Also see discussion in Sect. 7.3.
} 
cgs units, the equations of compressible MHD then are

$$
\begin{aligned}
\frac{\partial \varrho}{\partial t}+\nabla \cdot(\varrho \mathbf{v}) & =0, \\
\varrho\left(\frac{\partial \mathbf{v}}{\partial t}+(\mathbf{v} \cdot \nabla) \mathbf{v}\right) & =-\nabla p+\frac{1}{4 \pi}(\nabla \times \mathbf{B}) \times \mathbf{B}+\varrho \mathbf{g}, \\
\frac{\partial \mathbf{B}}{\partial t} & =\nabla \times(\mathbf{v} \times \mathbf{B})+\eta \nabla^{2} \mathbf{B}, \\
\nabla \cdot \mathbf{B} & =0, \\
\frac{\partial e}{\partial t}+\nabla \cdot\left(\left(e+p+\frac{B^{2}}{8 \pi}\right) \mathbf{v}-\frac{(\mathbf{v} \cdot \mathbf{B})}{4 \pi} \mathbf{B}\right) & =\frac{1}{4 \pi} \nabla \cdot(\mathbf{B} \times \eta \nabla \times \mathbf{B})+\varrho \mathbf{g} \cdot \mathbf{v}+Q_{\mathrm{rad}} .
\end{aligned}
$$

where $\mathrm{g}$ and $\eta$ are the gravitational acceleration and the magnetic diffusivity, respectively, $Q_{\text {rad }}$ is the radiative heating. For the results considered in this thesis, the magnetic diffusivity was not explicitely specified; the diffusive term $\eta \nabla^{2} \mathbf{B}$ is than dropped from equation and the diffusion of the magnetic field is described by artificial diffusivities (see Sect. 2.1.2).

The continuity equation, Eq. (2.1), and the divergence-free condition for the magnetic field, Eq. (2.4), are general. The derivation of the MHD approximations of the equation of motion, Eq. (2.2), the induction equation, Eq. (2.1.1), and the energy equation, Eq. (2.5) from the Navier-Stokes equation and the Maxwell equations makes use of the neglect of displacement currents (see, e. g. Biskamp 1997, Choudhuri 1998, for a formal derivation). On the right-hand side of the energy equation, Eq. (2.5), the source term for conductive heat transport does not appear because it is negligible in the photospheres and interiors of main-sequence stars (but plays a crucial role in the upper stellar atmospheric layers) and therefore is not considered in MURaM. Note that the MURaM approximations of the energy equation and the equation of motion do not include viscous dissipation. Analogous to the dissipation of the magnetic field, viscous dissipation is described by artificial diffusivities (see Sect. 2.1.2).

The system of equations, Eqs. (2.1) - (2.5), is closed by two equations of state,

$$
T=T(e, \varrho), \quad p=p(e, \varrho) .
$$

Owing to the ionisation of different species (most importantly of hydrogen), the ideal gas law does not apply for the plasma in the near-surface layers of stars. Therefore, Eq. (2.6) are complicated relations, which depend on the elemental composition of the star. In the MURaM code, they are approximated by interpolation between tabulated values from the OPAL equation-of-state (Rogers et al. 1996, Rogers 1994) for the solar composition published by Anders and Grevesse (1989).

\subsubsection{The numerical scheme}

The MURaM code solves numerical approximations to Eqs. (2.1) - (2.5) on a three-dimensional Cartesian grid. The spatial derivatives are discretised as fourth-order centred differences, and the time stepping is explicit (fourth-order Runge-Kutta solver). For more details, see Vögler (2003). 
As the resolved scales are several orders of magnitude larger than the scales on which the kinetic and magnetic energy is dissipated, the MURaM simulations are large-eddy simulations. For the solution of these it is necessary to introduce artificial diffusivities for all physical quantities calculated directly by the MHD scheme (i. e. for $\mathbf{v}, \mathbf{B}, e$, and $\varrho$ ), because the actual values of magnetic diffusivity and viscosity are much too small to affect these large scales. Without artificial diffusivities strong gradients in the velocity field and in advected quantities (e.g. in shock fronts) can cause numerical instabilities. The diffusivities depend on the resolution and are calculated in each time step for each coordinate direction and each quantity. The general procedure is described in Vögler (2003). Rempel et al. (2009) implemented a Minmod slope limiter for a later version of the MURaM code, which is also used for the simulations presented in this thesis.

\subsubsection{Treatment of radiation}

An important process in the photosphere is the interaction of the plasma with the radiation field. The radiative heating/cooling in the MURaM code is described by $Q_{\text {rad }}$ in the energy equation, Eq. (2.5). To calculate $Q_{\mathrm{rad}}$, the radiative heat transport within the simulation domain is solved after each time step of the MHD scheme.

The neglect of the displacement current has the consequence that MHD is not able to describe the interaction between electromagnetic waves and plasma motions. Radiation with frequencies $v$ fulfilling $2 \pi v \ll \omega_{p}$ with the plasma frequency $\omega_{p}=\left(4 \pi e^{2} n_{e} / m_{e}\right)^{1 / 2}$, can be treated by radiative MHD, because it only interacts with the plasma by emission and absorption and does not induce plasma oscillations. The typical value of $\omega_{p}$ in stellar atmospheres is of the order of $10^{11} \mathrm{~s}^{-1}$ and thus radiation with wavelengths $\lambda \ll 2 \mathrm{~cm}$ can be described without taking into account plasma oscillations. The bulk of the radiative flux in the stellar atmospheres simulated here is at wavelengths between $10^{-5}$ and $10^{-2} \mathrm{~cm}$.

The radiation field $I_{\lambda}$ in a medium is subject to absorption, scattering, and emission processes. The equation of radiative transfer along a given ray with coordinate $s$ then reads:

$$
\begin{aligned}
& \mathrm{d} I_{\lambda}=\left(-I_{\lambda}+S_{\lambda}\right) \kappa_{\lambda} \varrho \mathrm{d} s \text { or } \\
& \mathrm{d} I_{\lambda}=\left(-I_{\lambda}+S_{\lambda}\right) \mathrm{d} \tau_{\lambda} \text { with } \mathrm{d} \tau_{\lambda}=\kappa_{\lambda} \varrho \mathrm{d} s,
\end{aligned}
$$

where $S_{\lambda}$ is the source function, $\kappa_{\lambda}$ the opacity, $\varrho$ the density, and $\tau_{\lambda}$ the monochromatic optical depth; the index $\lambda$ signifies that these quantities depend on wavelength, $\lambda$. In MURaM, which assumes local thermodynamical equilibrium (LTE), the source function is given by the Planck function $B_{\lambda}(T)$ :

$$
S_{\lambda} \equiv B_{\lambda}(T)=\frac{2 h c}{\lambda^{5}} \frac{1}{\exp \left(\frac{h c}{\lambda k_{\mathrm{B}} T}\right)-1},
$$

where $h, k_{B}$, and $c$ are the Planck constant, the Boltzmann constant, and the (vacuum) speed of light, respectively. With this assumption, $S_{\lambda}$ becomes independent of the radiation field and is only a function of $T$ and $\lambda$, which facilitates the formal solution of the radiative transfer equation for single wavelengths and ray directions.

A fully wavelength- and angle-resolved treatment of radiation in every time step of a three-dimensional simulation is impossible with today's computational power. The MURaM 
code uses the short characteristics scheme (Kunasz and Auer 1988), which approximates the intensity field for a small number of directions by calculating the intensity reaching each grid point $P$ from the nearest intersection $Q$ with a cell boundary in the (upstream) direction of the ray. For this ray, the radiative transfer equation, Eq. (2.8) is integrated, giving

$$
I_{P}=I_{Q} \exp \left(-\tau_{Q}\right)+\int_{0}^{\tau_{Q}} B(\tau) \exp (-\tau) \mathrm{d} \tau
$$

where $\tau$ is the optical depth along the ray from $P$ to $Q$,

$$
\tau=\int_{P}^{R} \kappa(s) \varrho(s) \mathrm{d} s
$$

with a point $R$ on the ray between $P$ and $Q ; \tau_{Q}$ is this optical depth $\tau$ for $R=Q$. In the current implementation of MURaM, the A4 scheme of Carlson (1963), which uses three rays per octant, is used. The intensity field is calculated for these 24 directions by stepping through the grid downstream. Due to the necessary interpolation to obtain $I_{Q}, B(\tau), \kappa(s)$ and $\varrho(s)$, the procedure entails some numerical diffusion, but is much more efficient than a long-characteristics solution.

The wavelength dependence of radiation is approximated with an opacity binning method (Nordlund 1982); for a detailed description of the MURaM implementation see Vögler (2003), Vögler et al. (2004). For the simulations considered here, the opacity distribution functions from the ATLAS9 package (Kurucz 1993) were used as basis for the binning. Four opacity bins were calculated using the $\tau$-sorting procedure. In this method, wavelengths are binned together according to a reference optical depth level at which the vertical optical depth at this wavelength reaches unity. As reference optical depth, a hybrid optical depth, $\bar{\tau}$, was used: $\bar{\tau}$ converges to the Rosseland optical depth for large optical depth and to the Planck optical depth for small optical depth with a smooth transition centred at $\tau_{0}=0.35$ (cf. Ludwig 1992, Vögler 2003). The threshold levels for the bins are at $\log \bar{\tau}=0,-2$, and -4 . The reference atmosphere needed for the $\tau$-sorting was obtained as follows. For preliminary simulations for F3V, G2V, and K0V stars (for the parameters of these simulations, see Sect. 2.3.1), the solar opacity bins, which were available from earlier studies (e. g. Beeck et al. 2012), were used. The horizontally averaged simulation results were then used as new reference atmospheres to generate improved binned opacities, which were then used for the final simulations of the F3V, G2V, and K0V stars considered here. ${ }^{2}$ For the K5V, M0V, and M2V stars, the improved binned opacities of the K0V star were used for the preliminary simulations. The results of these simulations were then used as reference atmospheres to obtain the binned opacities for the final simulations.

\footnotetext{
${ }^{2}$ This procedure could be iterated. However, even after this one "iteration", the changes in the horizontally averaged temperature structure were small and no further iteration was made.
} 
Table 2.1: Effective temperature and gravitational acceleration of the 24 simulations

\begin{tabular}{lccccc}
\hline \hline SpT & $\log g\left[\mathrm{~cm} \mathrm{~s}^{-2}\right]$ & \multicolumn{4}{c}{$T_{\text {eff }}$} \\
& & hydro. & $B_{0}=20 \mathrm{G}$ & $B_{0}=100 \mathrm{G}$ & $B_{0}=500 \mathrm{G}$ \\
\hline F3V & 4.301 & $(6893 \pm 7) \mathrm{K}$ & $(6885 \pm 6) \mathrm{K}$ & $(6911 \pm 8) \mathrm{K}$ & $(7003 \pm 5) \mathrm{K}$ \\
G2V & 4.438 & $(5780 \pm 13) \mathrm{K}$ & $(5779 \pm 9) \mathrm{K}$ & $(5802 \pm 8) \mathrm{K}$ & $(5864 \pm 9) \mathrm{K}$ \\
K0V & 4.609 & $(4856 \pm 5) \mathrm{K}$ & $(4858 \pm 2) \mathrm{K}$ & $(4878 \pm 4) \mathrm{K}$ & $(4901 \pm 2) \mathrm{K}$ \\
$\mathrm{K} 5 \mathrm{~V}$ & 4.699 & $(4368 \pm 2) \mathrm{K}$ & $(4376 \pm 2) \mathrm{K}$ & $(4383 \pm 3) \mathrm{K}$ & $(4402 \pm 2) \mathrm{K}$ \\
M0V & 4.826 & $(3905 \pm 1) \mathrm{K}$ & $(3907 \pm 1) \mathrm{K}$ & $(3909 \pm 1) \mathrm{K}$ & $(3906 \pm 1) \mathrm{K}$ \\
M2V & 4.826 & $(3690 \pm 1) \mathrm{K}$ & $(3691 \pm 1) \mathrm{K}$ & $(3692 \pm 1) \mathrm{K}$ & $(3679 \pm 1) \mathrm{K}$
\end{tabular}

\subsection{The SPINOR code}

In Sect. 6 the effect of the three-dimensional atmospheric structure and of the magnetic field on the profiles of spectral lines was analysed. The SPINOR code was used to forwardsynthesise the Stokes vector,

$$
\mathbf{I}(\lambda)=(I(\lambda), Q(\lambda), U(\lambda), V(\lambda))^{T},
$$

from the simulated stellar atmospheres. The components of the Stokes vector correspond to the total monochromatic intensity, $I_{\lambda}$, the intensity of circularly polarised, $V_{\lambda}$, and of linearly polarised light (two directions, rotated by $45^{\circ}$ ), $Q_{\lambda}$ and $U_{\lambda}$.

For the results presented in Sect. 6 only the forward part, STOPRO, of SPINOR was used. It solves the radiative transfer equation for polarised radiation, which reads

$$
\frac{\mathrm{d} \mathbf{I}(\lambda)}{\mathrm{d} s}=-\underline{\underline{K}}(\lambda) \mathbf{I}(\lambda)+\mathbf{j}(\lambda),
$$

where $\underline{K}(\lambda)$ is the (opacity related) absorption matrix and $\mathbf{j}(\lambda)$ is the emission vector (containing a generalisation of the source function). The numerical solution of Eq. 2.13 is discussed in detail in Frutiger (2000); for the spectral line profiles presented in this thesis, the Diagonal Element Lambda Operator (DELO; Rees et al. 1989) method was chosen in STOPRO.

In this thesis, only the Stokes-I component is considered. The other Stokes components were simultaneously calculated for the same MURaM cubes and will be analysed in future work as discussed in Sects. 7.2 and 7.3.

\subsection{Simulation setup}

\subsubsection{Stellar parameters}

In this thesis, the results of 24 simulation runs are presented. Six of these are nonmagnetic (hydrodynamic) simulations, which also serve as reference for the analysis of the effects of the magnetic field and are used as initial condition for the simulation runs with magnetic field. The simulations have parameters corresponding to a set of mainsequence stars with solar metallicity. The only parameters varied were the gravitational acceleration, $g$, at the surface (assumed constant throughout the simulation box), and the 


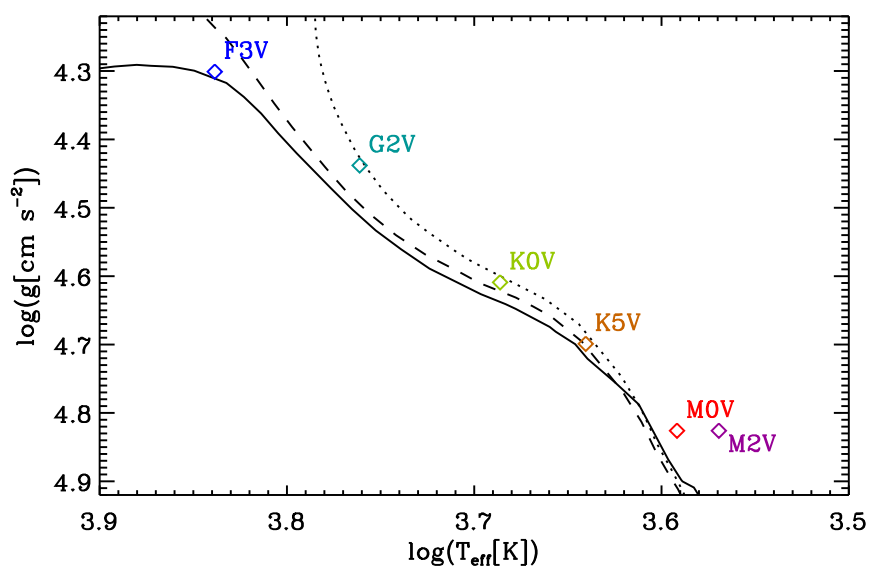

Figure 2.1: $\log T_{\text {eff- }} \log g$ diagram of the six hydrodynamic simulations along with three isochrones by Bressan et al. (2012): zero-age main sequence (solid), $1 \mathrm{Ga}$ (dashed), and 4.5 Ga (dotted).

effective temperature, $T_{\text {eff }}$, which was indirectly determined by adapting the entropy density of the inflowing plasma (also see Sect. 2.3.2). The values of $T_{\text {eff }}$ and $\log g$ are given in Table 2.1. Figure 2.1 shows the position of the stellar parameters in a $\log T_{\text {eff }}-\log g$ diagram along with three isochrones by Bressan et al. (2012).

For each of the six hydrodynamic simulations, there exist three magnetic (magnetohydrodynamic) simulations (see Sect. 2.3.3) with different average field strengths, but almost the same stellar parameters ( $T_{\text {eff }}$ slightly differs between cases of different magnetic field, see Table 2.1 and Fig. 2.2).

\subsubsection{Setup of the non-magnetic simulations}

The simulation domain of MURaM corresponds to a rectangular box containing a small horizontal section of the topmost layers of the convective envelope of the star and the lower part of the stellar atmosphere ( $\approx$ the photosphere). In order to facilitate the comparison between simulations of different stars, the dimensions of the box were chosen such that the expected number of granules at a given time in the simulation box as well as the number of pressure scale heights below and above the optical surface are approximately equal in all cases. The horizontal resolution was $512 \times 512$ cells in all simulations. The vertical resolution varies between simulations for different spectral type (but not between simulations runs for the same spectral type) because it was chosen sufficiently high to resolve the steep photospheric temperature gradient and the pressure stratification (requiring $\Delta z<H_{p} / 5$, everywhere). The box sizes and resolutions are summarised for all simulations in Table 2.2.

The effective temperature of the simulation boxes is controlled by the entropy density of the inflows at the lower boundary of the simulation domain. The automatic temperature control implemented in MURaM (see Vögler 2003) was switched off for all simulation runs presented in this thesis. Instead, the entropy density of the inflows was manually varied 
Table 2.2: Box sizes and grid resolutions. ${ }^{3}$

\begin{tabular}{lcccccc}
\hline \hline Simulation & F3V & G2V & K0V & K5V & M0V & M2V \\
\hline Box height $[\mathrm{Mm}]$ & 9 & 3 & 1.8 & 1.5 & 0.9 & 0.8 \\
above $z_{0}{ }^{a}$ & 1.57 & 0.95 & 0.48 & 0.41 & 0.25 & 0.21 \\
below $z_{0}$ & 7.43 & 2.05 & 1.32 & 1.09 & 0.65 & 0.59 \\
\hline \# of pressure scale heights & 13.1 & 14.0 & 13.2 & 15.3 & 14.8 & 14.5 \\
above $z_{0}$ & 6.9 & 8.6 & 7.1 & 9.1 & 8.4 & 7.8 \\
below $z_{0}$ & 6.2 & 5.4 & 6.2 & 6.4 & 6.4 & 6.8 \\
\hline$H_{p}$ at $z_{0}[\mathrm{~km}]$ & 500 & 200 & 90 & 65 & 38 & 35 \\
\hline$\Delta z^{\mathrm{b}}[\mathrm{km}]$ & 11.25 & 10 & 6 & 5 & 4 & 3.2 \\
$\min \left(H_{p}\right) / \Delta z$ & 18.1 & 10.0 & 9.57 & 7.87 & 6.53 & 7.19 \\
\hline Horizontal box size $[\mathrm{Mm}]$ & 30 & 9 & 6 & 4 & 2.5 & 1.56 \\
\hline$\Delta x, \Delta y{ }^{\mathrm{c}}[\mathrm{km}]$ & 58.6 & 17.6 & 11.7 & 7.81 & 4.88 & 3.05 \\
$\Delta x / \Delta z$ & 5.21 & 1.76 & 1.95 & 1.56 & 1.22 & 0.953 \\
\hline \multirow{2}{*}{$z_{0}=\left\langle z\left(\tau_{\mathrm{R}}=1\right)\right\rangle$} & \multicolumn{7}{c}{} & & \\
b $\Delta z$ is the vertical grid resolution \\
c $\Delta x$ and $\Delta y$ are the horizontal grid resolution; in all simulations considered here, \\
$\Delta x=\Delta y$ was chosen
\end{tabular}

until the desired effective temperature was reached. After that, the simulations were run for several hours of simulated time (comparable to the Kelvin-Helmholtz times of the boxes), before six snapshots of each simulation were selected for the analysis presented in this thesis. One of these snapshots for each spectral type was also used as an initial condition for all magnetic runs of the same spectral type.

\subsubsection{Setup of the magnetic simulations}

For the analysis of the magnetoconvection in the different simulated stars, snapshots of the simulations described in Sect. 2.3.2 were used as initial conditions for $\varrho(x, y, z), e(x, y, z)$, and $\mathbf{v}(x, y, z)$. As initial condition for the field,

$$
B_{x}(x, y, z) \equiv 0, \quad B_{y}(x, y, z) \equiv 0, \quad B_{z}(x, y, z) \equiv B_{0}
$$

was applied. For each star, simulations with $B_{0}=20 \mathrm{G}, B_{0}=100 \mathrm{G}$, and $B_{0}=500 \mathrm{G}$ were run. Owing to the interaction with the flows, the field configuration evolves from the arbitrary initial condition of Eq. (2.14) to a statistically stationary configuration within a time of the order of the convective turnover time (see Sect. 3.2).

The sudden "injection" of a magnetic field in the existing self-consistently structured atmosphere can lead to short-lived transient phenomena (e.g. additional pulsations), which die away after some time. Moreover, the presence of the field changes the atmospheric structure (see Sect. 3.3), which needs some time to reach a statistically stationary

\footnotetext{
${ }^{3}$ This table has been published as Table 2 in Beeck et al. (2013a)
} 

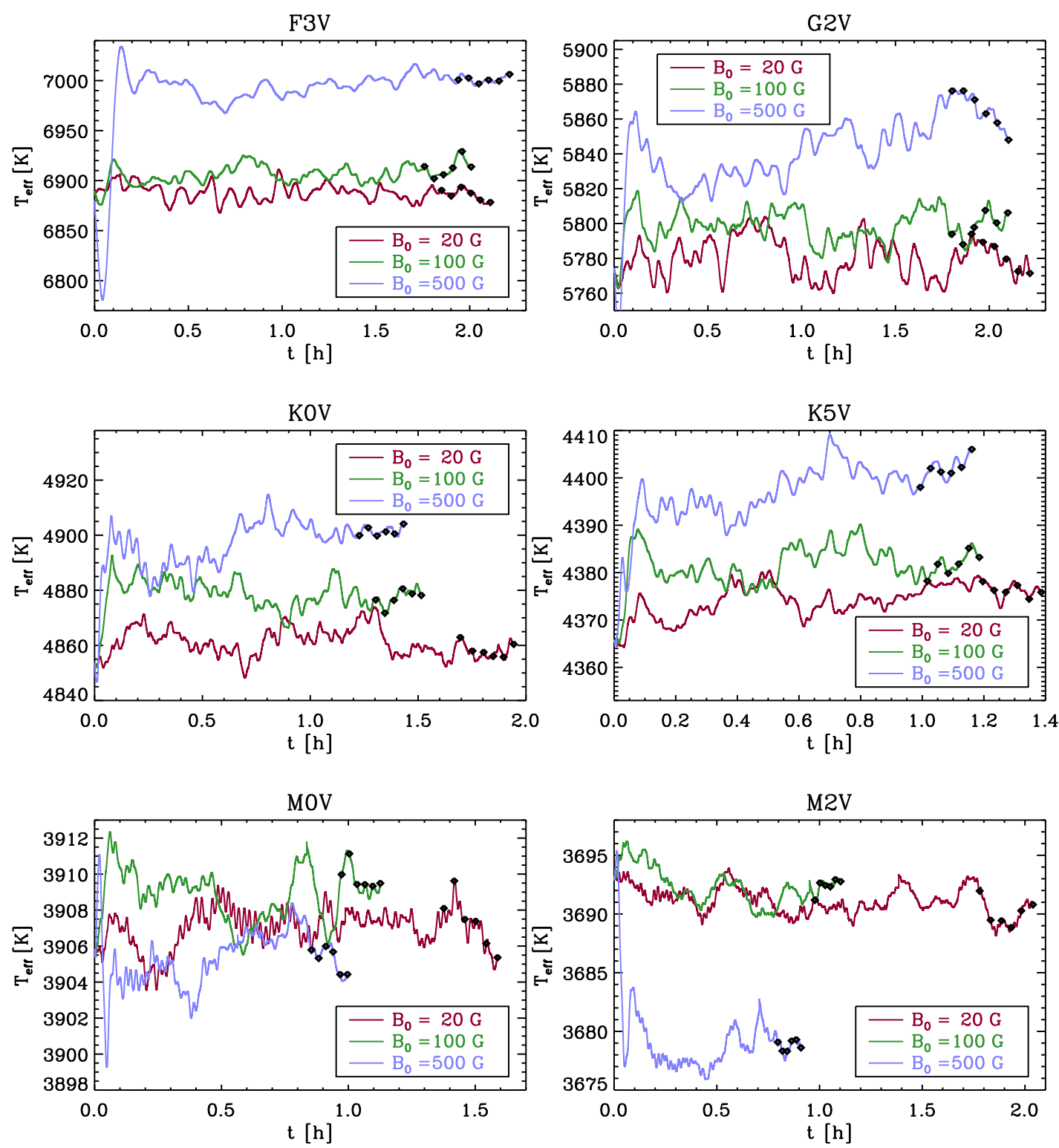

Figure 2.2: Temporal evolution of the effective temperature of the magnetic simulation runs after the injection of the magnetic field (at $t=0$ ). The small black diamonds mark the six snapshots of each simulation run which are the basis for the analysis in this thesis.

state. Therefore, the snapshots of the magnetic runs analysed in the later sections of this thesis were taken roughly one to two hours of simulated stellar time after the injection of the field. Various quantities were inspected to verify that the simulations have stabilised at this point in time.

Figure 2.2 shows the temporal evolution of the effective temperatures for all magnetic runs. Especially the $500 \mathrm{G}$ runs show a significant change of the effective temperature after the field injection. After only a few minutes the temperature quasi-stabilises (with the usual temporal fluctuations). Owing to the formation of flux concentrations which appear as bright or dark structures (cf. Sect. 3.3), the effective temperature of the quasi-stable 

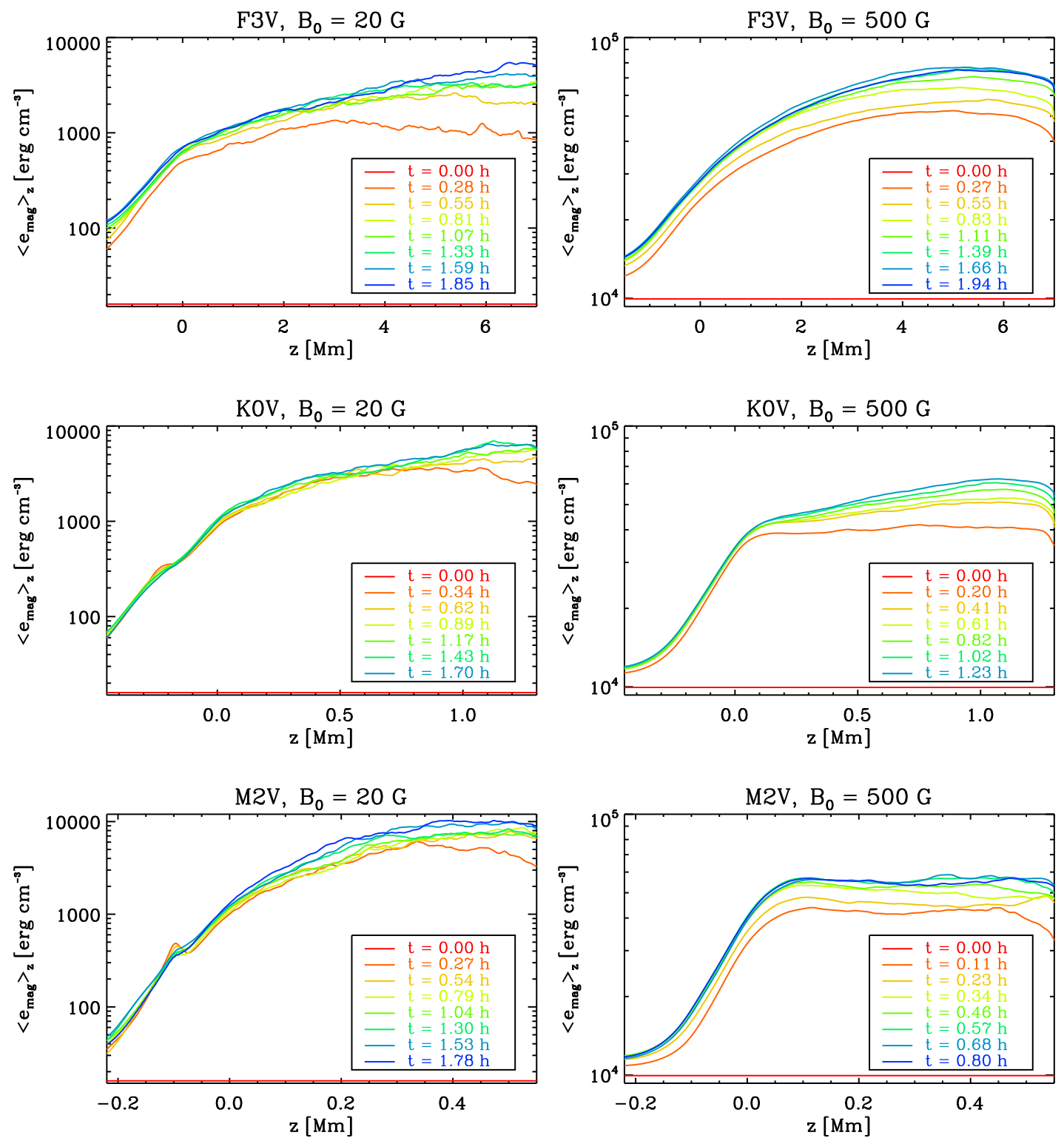

Figure 2.3: Temporal evolution of the depth profile of the magnetic energy density $\left\langle e_{\text {mag }}\right\rangle_{z}$, averaged over horizontal planes, in some magnetic runs after the field injection (at $t=0$ ). The last time given in each plot corresponds to the time step of the first of the six snapshots for the respective simulation run which are the basis for the analysis in this thesis.

convection is not the same for different values of $B_{0}$. The changes in $T_{\text {eff }}$ of the order of up to 1-2\% correspond to a change in the energy flux through the top of the box of a few percent, which has to be compensated by a change in the energy flux through the bottom. However, the finite heat capacity in the box adds some thermal inertia: the energy balance is adapting to the new atmospheric structure on time scales up to the Kelvin-Helmholtz timescale $\tau_{\mathrm{KH}}$. For our simulation boxes, $\tau_{\mathrm{KH}}$ is between 9 hours $(\mathrm{F} 3 \mathrm{~V})$ and 14 days $(\mathrm{M} 2 \mathrm{~V})$. This implies that the simulation boxes show a transient thermodynamical structure: only after several Kelvin-Helmholtz times of the box the energy balance between 
the convective input (at the bottom boundary) and radiative output (at the top boundary) is fully restored. As real surface magnetism on the Sun (and probably also on stars) entails surface structure variations on time scales much shorter than $\tau_{\mathrm{KH}}$ of the convective envelope, this equilibrium on $\tau_{\mathrm{KH}}$ is, however, probably irrelevant for the phenomena analysed in this thesis. The small black diamonds in the figure correspond to the times (and effective temperatures) of the six snapshots, on which the analysis in later sections of this thesis is based. There temporal separation is on the order of several minutes and much shorter than $\tau_{\mathrm{KH}}$, so there are no obvoius trends in effective temperature and in the thermodynamics atmosphere structure.

Figure 2.3 shows the evolution of the profile of the horizontally averaged magnetic energy density, $\left\langle e_{\mathrm{mag}}\right\rangle_{z}$, for a selection of different simulations. At the field injection the magnetic energy density is $B_{0}^{2} /(8 \pi)$ everywhere, following from the initial condition of $\mathbf{B}$, Eq. (2.14). The vertical magnetic flux is advected horizontally by the diverging convective outflows and becomes concentrated in the downflows (cf. Sect. 3.2). This concentration enhances the magnetic energy density, especially in the runs with low average field strength. After some time (roughly $1 \mathrm{~h}$ of stellar time) a quasi-stationary state is reached. As visible in Fig. 2.3, in some runs (e.g. the $500 \mathrm{G}$ run of the K0V star) there might still a weak growth of $e_{\text {mag }}$ in the deepest layers, while in most runs there is no apparent trend left. The last time step for which a curve is plotted in the figure corresponds to the time of the first snapshot analysed in later sections of this thesis. 


\section{Overall structure}

\subsection{Overall structure of the non-magnetic simulations ${ }^{1}$}

\subsubsection{General morphology of near-surface convection}

Figure 3.1 gives maps of the bolometric intensity emerging vertically from the simulated stellar surfaces for single snapshots of the time-dependent simulations. All simulations show intensity patterns reminiscent of solar granulation. The typical size of the granules varies from $\sim 5 \mathrm{Mm}$ for $\mathrm{F} 3 \mathrm{~V}$ to $\sim 0.3 \mathrm{Mm}$ for $\mathrm{M} 2 \mathrm{~V}$. The rms bolometric intensity contrast (denoted by $\sigma_{I}$ in Table 3.1) decreases from about 20\% for F3V to less than 3\% in the $\mathrm{M} 2 \mathrm{~V}$ simulation, reflecting decreasing temperature fluctuations on surfaces of constant optical depth (see Sect. 3.1.3).

There are qualitative changes in the visual appearance of the surface convection along the sequence of simulated stars. For instance, the granulation pattern of the F3V model appears "rough" and irregular owing to numerous shock waves at the optical surface. Shocks are rarer and weaker in the near-surface layers of the cooler stars since the typical convective velocities are lower (also in relation to the sound speed; cf. Fig. 3.5). At the cool end of our model sequence, the M-dwarf granules, which are sustained by the slowest convective flows, have more irregular shapes but less brightness substructure than their counterparts on the simulated G- and K-type stars. As we report quantitatively in Sect. 4.2.1, their dark intergranular lanes are thinner (with respect to the granule size) and vary more strongly in intensity and width than those of the other stars (see also Ludwig et al. 2002).

Ludwig et al. (2006) found "dark knots" associated with strong downflows and vortex motion in simulations of convection in M-type main- and pre-main-sequence objects. Our simulations show knots of high vorticity associated with strong downflows in all models (some examples in Fig. 3.1 are: $\mathrm{G} 2 \mathrm{~V},(x, y)=(8.7 \mathrm{Mm}, 4.4 \mathrm{Mm})$; K5V, $(x, y)=(0.36 \mathrm{Mm}, 0.52 \mathrm{Mm}) ; \mathrm{M} 0 \mathrm{~V},(x, y)=(0.45 \mathrm{Mm}, 0.6 \mathrm{Mm}))$. They become increasingly stable and prominent at lower effective temperatures. In our models, some of these vortices are evacuated strongly enough by the effect of the centrifugal force to become brighter than their surroundings (cf. vortices in solar simulations studied by Moll et al. 2011, 2012). Most frequently these bright vortex structures occur in our two K-type simulations.

A more detailed analysis of the granulation properties and their effects on spectral lines is given in Sects. 4 and 6.

\footnotetext{
${ }^{1}$ This section has been published as Section 3 of Beeck et al. (2013a)
} 
Table 3.1: Stellar parameters, bolometric intensities and rms velocities.

\begin{tabular}{|c|c|c|c|c|c|c|}
\hline \multirow[t]{2}{*}{ Simulation } & \multirow{2}{*}{$\log g^{\mathrm{a}}$} & \multirow[t]{2}{*}{$T_{\text {eff }}[\mathrm{K}]$} & \multirow{2}{*}{ 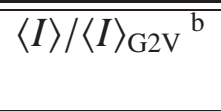 } & \multirow[t]{2}{*}{$\sigma_{I}^{\mathrm{c}}[\%]$} & \multicolumn{2}{|c|}{$\overline{v_{z, \mathrm{rms}}(z)\left[\mathrm{km} \mathrm{s}^{-1}\right]}$} \\
\hline & & & & & $z=z_{0}{ }^{\mathrm{d}}$ & $z=z_{2}{ }^{\mathrm{e}}$ \\
\hline F3V & 4.301 & $6893.2 \pm 6.4$ & 1.98 & 20.3 & 5.1 & 3.5 \\
\hline G2V & 4.438 & $5764.4 \pm 7.4$ & 1.00 & 15.7 & 2.0 & 1.2 \\
\hline K0V & 4.609 & $4855.6 \pm 5.5$ & 0.500 & 8.04 & 1.1 & 0.72 \\
\hline $\mathrm{K} 5 \mathrm{~V}$ & 4.699 & $4367.9 \pm 2.0$ & 0.322 & 6.90 & 0.99 & 0.57 \\
\hline MOV & 4.826 & $3904.8 \pm 0.8$ & 0.195 & 3.55 & 0.72 & 0.42 \\
\hline M2V & 4.826 & $3688.6 \pm 1.4$ & 0.153 & 2.17 & 0.59 & 0.57 \\
\hline \multicolumn{7}{|c|}{ a $g$ is the gravitational acceleration at the stellar surface in cgs units. } \\
\hline \multicolumn{7}{|c|}{ temporal and spatial mean of the bolometric intensity normalised to the solar value } \\
\hline \multicolumn{7}{|c|}{ c bolometric intensity contrast (temporal mean) } \\
\hline \multicolumn{7}{|c|}{${ }^{\mathrm{d}} z_{0}:=\left\langle z\left(\tau_{\mathrm{R}}=1\right)\right\rangle$} \\
\hline$z_{2} \mathrm{i}$ & & & & & & \\
\hline
\end{tabular}

\subsubsection{Velocity field}

As the visible granulation pattern is created by convective flows, it is strongly correlated to the vertical velocities at the optical surface, $v_{z}(z=0)$. Figure 3.2 shows $v_{z}(z=0)$ for four of the six simulations. The snapshots are taken at the same time as in Figure 3.1. The colour scale of the images saturate at $2 v_{z, \text { rms }}\left(z_{0}\right)$ with $z_{0}:=\langle z\rangle_{\tau_{\mathrm{R}}=1}$, values of which are given in Table 3.1. The granules visible in Figure 3.1 correspond to upflows, while the dark intergranular lanes correspond to downdrafts. In the G-, K-, and M-type simulations, an anti-correlation between size and mean upflow velocity of the granules is indicated: while most of the small convection cells appear (almost) saturated in Figure 3.2, meaning their velocity reaches $2 v_{z, \text { rms }}\left(z_{0}\right)$, the larger granules appear paler, meaning their upflow speed is lower. In the F3V simulation, this effect is not visible, due to a strong largescale modulation of the vertical velocity at the optical surface. This large scale pattern might hint to a strong mesogranulation in this spectral type. Unfortunately, the length scale of this modulation is the horizontal box size, which raises the question whether this effect is produced, enhanced, or modified by the periodic boundary condition. A test simulation with a box twice as large has shown similar but weaker large-scale modulation but probably is still strongly influenced by the periodic boundary condition.

Figure 3.3 shows maps of the vertical velocity at a depth of 4.6 pressure scale heights below the optical surface, where the average pressure is 100 times the average pressure at the optical surface, $p_{0}:=\langle p\rangle_{\tau_{\mathrm{R}=1}}$. The typical size of the convection cells is significantly larger at this depth than at the surface. A rough estimate based on mass conservation and stationarity predicts a proportionality between the horizontal scale of the vertical velocity pattern at a given depth, $D_{\text {hor }}$, and the local density scale height, $H_{\varrho}$ (Nordlund et al. 2009),

$$
D_{\mathrm{hor}}=4 H_{\varrho}\left(\frac{v_{\mathrm{hor}}}{v_{\mathrm{ver}}}\right)
$$



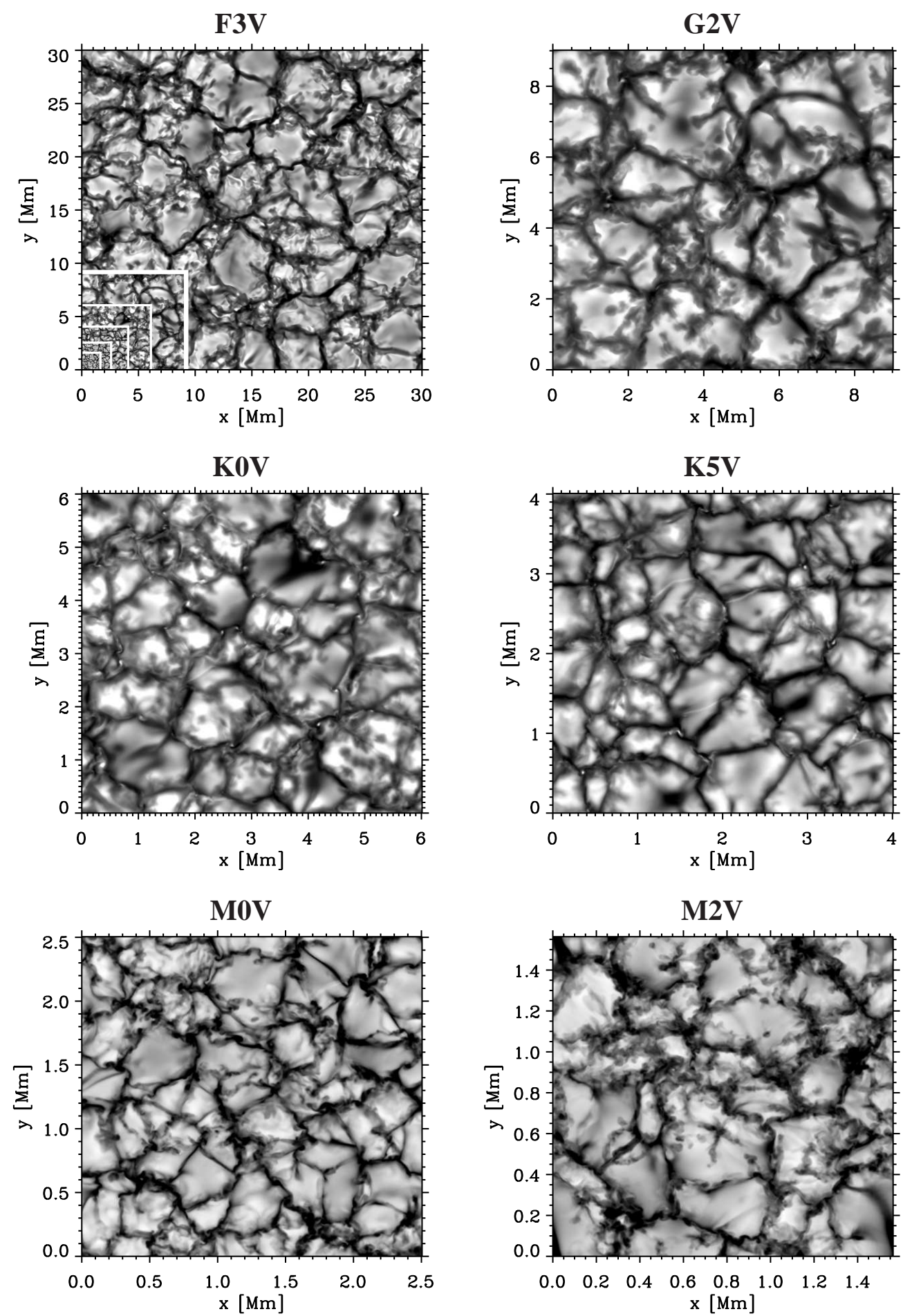

Figure 3.1: Maps of bolometric intensity emerging vertically from single snapshots of the six simulations. The grey scale of each image is saturated at $\pm 2 \sigma_{I}$, where $\sigma_{I}$ is the rms contrast (cf. Table 3.1). The significant difference in the length scales of the images is illustrated by the inset in the upper left panel, which shows all other images on the same scale as the one from the F3V simulation. 
F3V

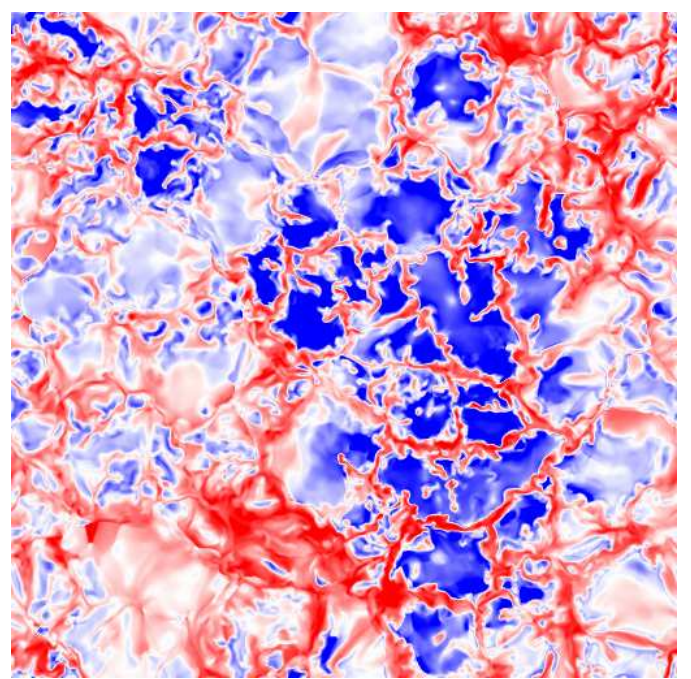

KOV

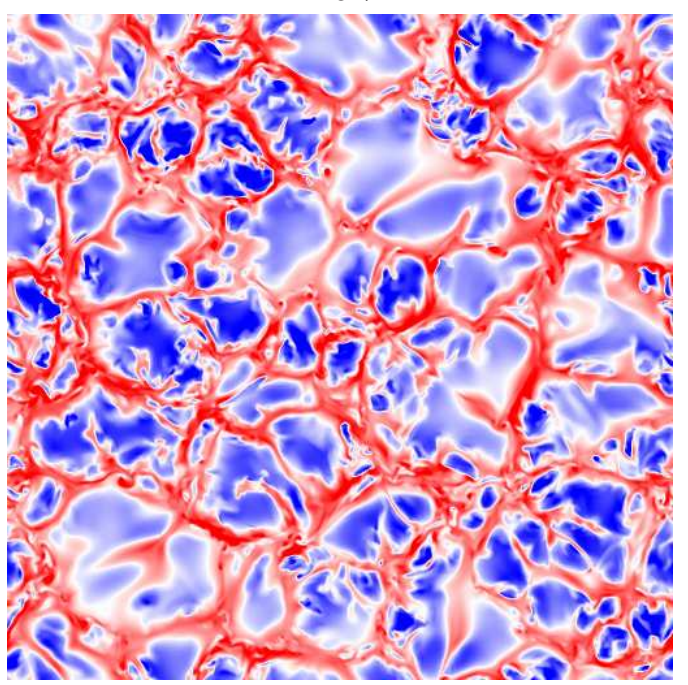

G2V

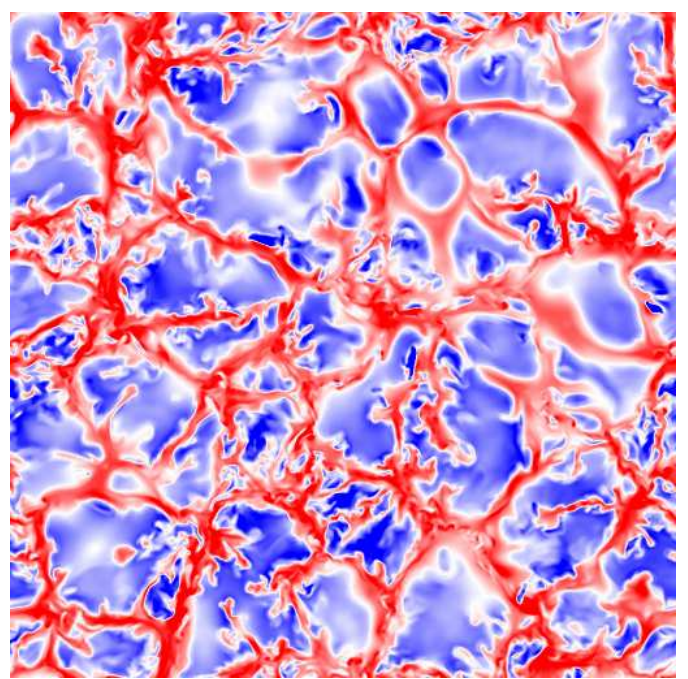

MOV

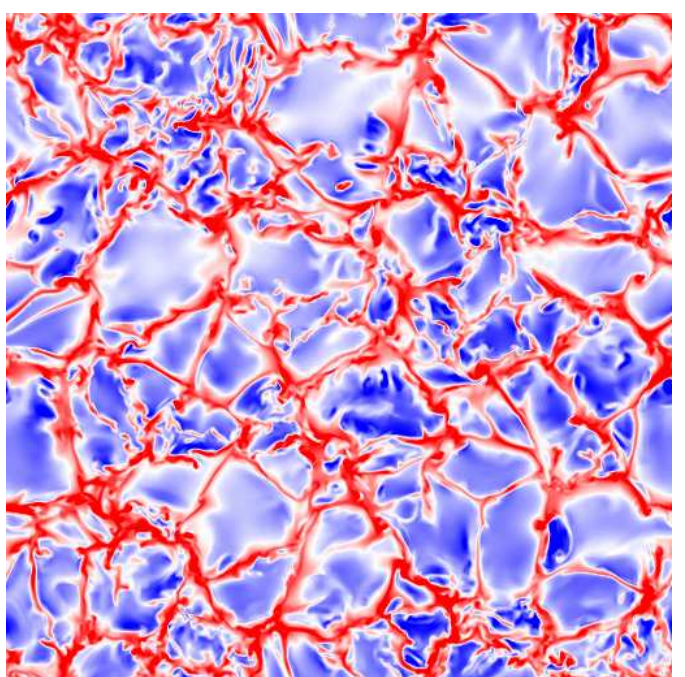

Figure 3.2: Maps of the vertical velocity $v_{z}$ at constant geometrical depth $z_{0}=\left\langle z\left(\tau_{\mathrm{R}}=1\right)\right\rangle$ (average level of the optical surface) for four of the six models. Upward motions are blue, downward motions are red, colour scales saturate at $\pm 2 \cdot v_{z, \text { rms }}\left(z_{0}\right)$ (for values, see Table 3.1). Note that the horizontal scales are different (cf. Fig. 3.1).

where $v_{\text {hor }}$ and $v_{\text {ver }}$ are the horizontal and vertical convection velocities, respectively. They can be approximated by the horizontally averaged (height-dependent) rms values of the vertical and horizontal components of the fluid velocity,

$$
v_{\mathrm{ver}} \approx v_{z, \mathrm{rms}}:=\sqrt{\left\langle v_{z}^{2}\right\rangle_{z}}
$$

and

$$
v_{\text {hor }} \approx v_{x, y, \text { rms }}:=\sqrt{\left\langle v_{x}^{2}+v_{y}^{2}\right\rangle_{z}},
$$

respectively (for the definition and discussion of the horizontal average $\langle\cdot\rangle_{z}$, see Appendix A). In the left panel of Figure 3.4 the ratio of $v_{x, y, r m s}$ and $v_{z, \text { rms }}$ is plotted as a 
F3V

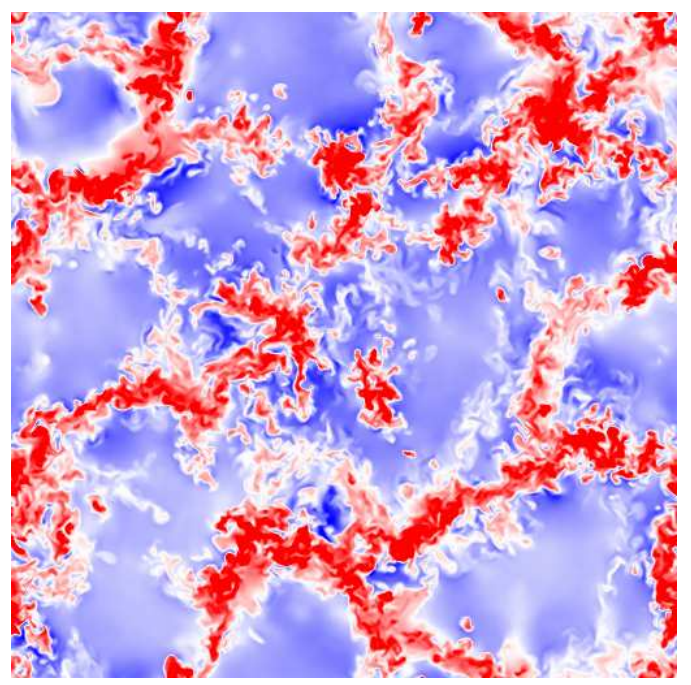

KOV

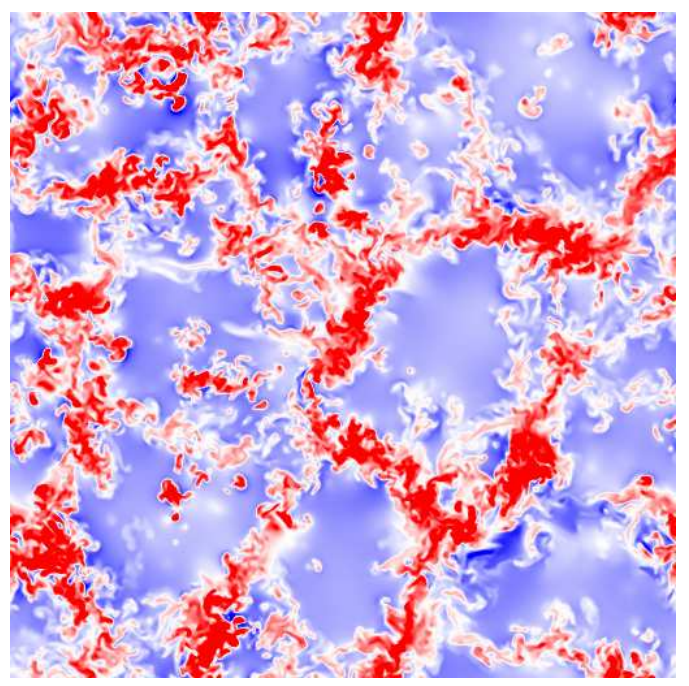

G2V

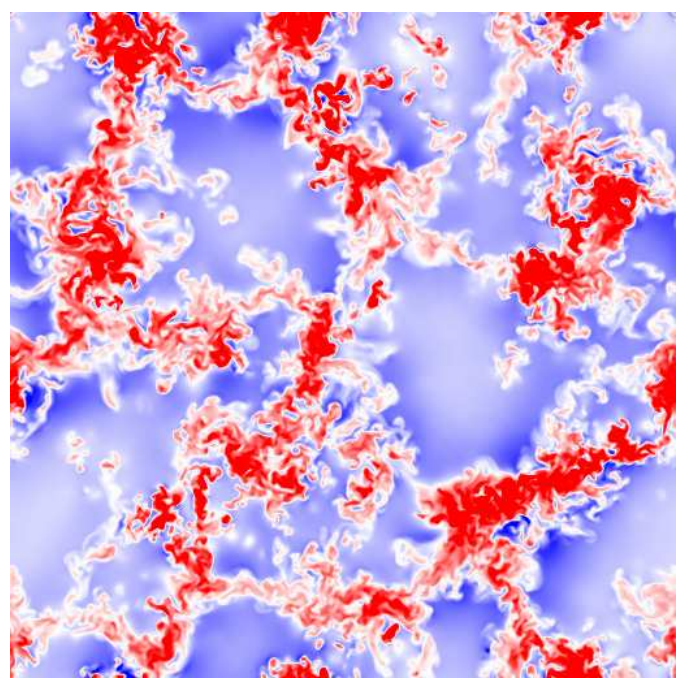

MOV

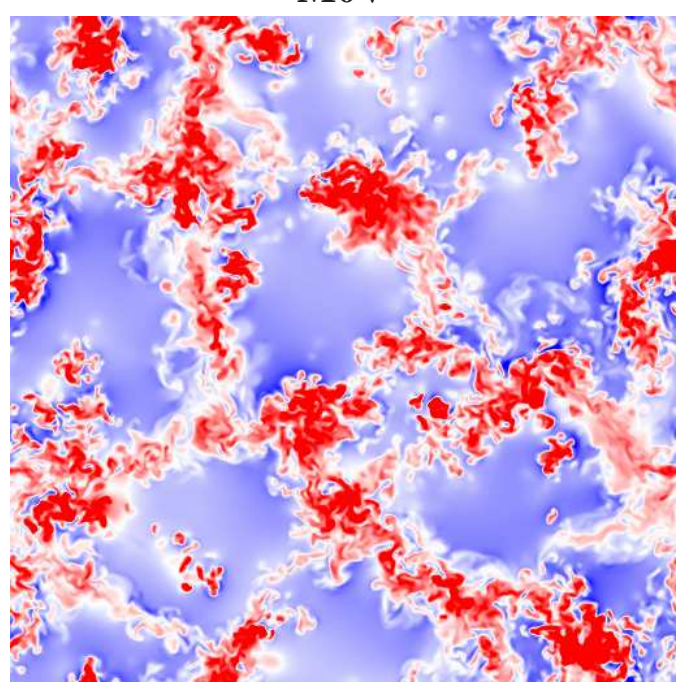

Figure 3.3: Same as Figure 3.2, but for geometrical depth $z_{2}$ with $\left\langle p\left(z_{2}\right)\right\rangle=100\left\langle p\left(z_{0}\right)\right\rangle$, corresponding to 4.6 pressure scale heights below the optical surface.

function of normalised gas pressure $\langle p\rangle_{z} / p_{0}$. In subsurface layers, we find $v_{\text {hor }} / v_{\text {ver }} \approx 1$, hence Eq. (3.1) predicts that the horizontal scale of the flow pattern roughly follows the trend of the inwardly increasing density scale height.

The right panel of Figure 3.4 shows the profile of $D_{\text {hor }}$ as derived from Eq. (3.1) in units of the horizontal box size $X_{\text {tot }}$ of the respective simulation. The density scale height obtained from the simulations was smoothed (convolution with a Gaussian kernel $\sigma=$ $10 \Delta z$ ) to avoid a sharp maximum of $D_{\text {hor }}$ at the optical surface of the two hottest models (cf. Fig. 3.8). The predicted horizontal scale of $12-20 \%$ of the horizontal box size at the optical surface and $25-40 \%$ of the horizontal box size at $p=100 \cdot p_{0}$ matches the sizes of the patterns visible in Figures 3.2 and 3.3.

The left panel of Figure 3.5 shows the depth dependence of $v_{z, \text { rms }}$, which is a measure of the typical convective velocity. The profiles of $v_{z, \text { rms }}$ all peak near the optical surface, 

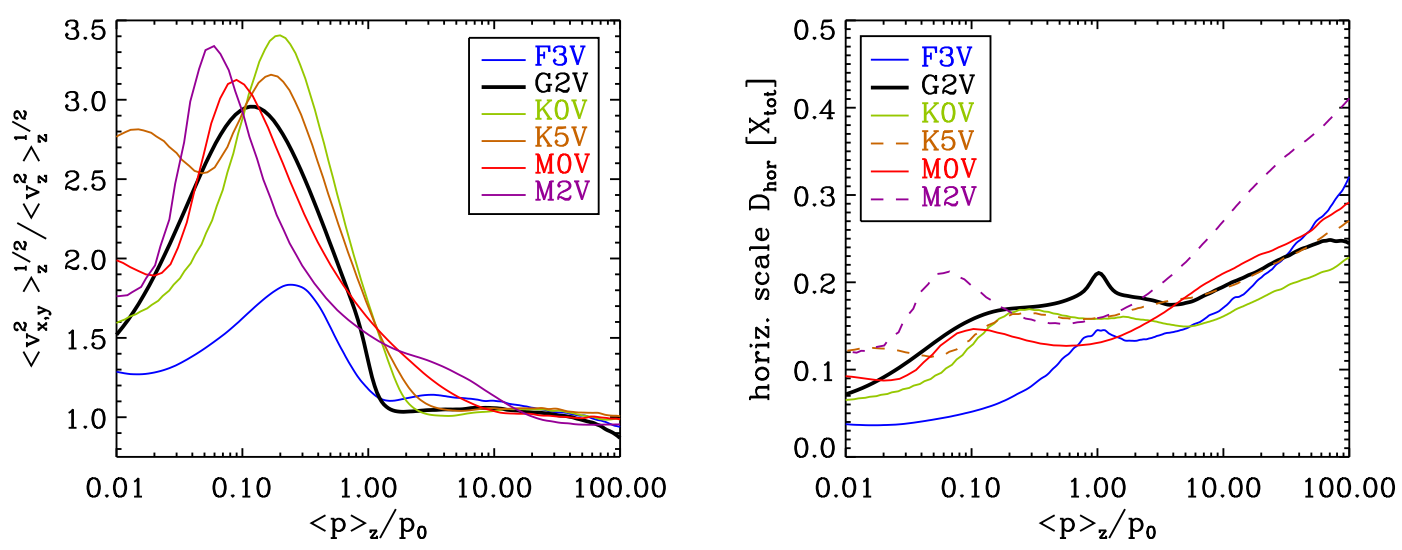

Figure 3.4: Properties of the convective flows. Left: ratio of rms values of horizontal and vertical flow velocity on surfaces of constant geometrical depth. Right: estimated horizontal scale of the vertical velocity patterns as derived from Eq. (3.1) on surfaces of constant geometrical depth. The horizontal scale is given in units of the horizontal box size, $X_{\text {tot }}$, for an easier comparison with Figs. 3.2 and 3.3. The solid curves refer to the four simulations shown in these figures, the dashed curves to the remaining two simulations.
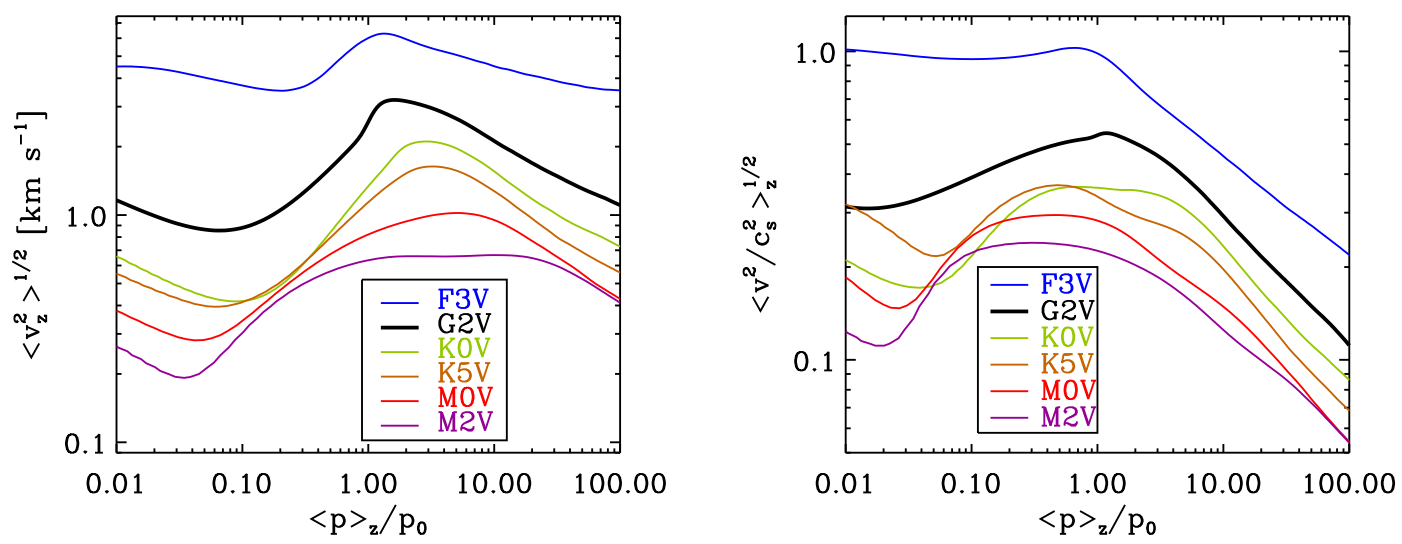

Figure 3.5: Flow velocity rms. Left: rms of the vertical component of the flow velocity on surfaces of constant geometrical depth. Right: rms of the modulus of the flow velocity in units of the local sound speed, $c_{s}$, (Mach number) on surfaces of constant geometrical depth.

where radiative energy transport starts to become important (cf. Fig. 3.12). The peak rms velocity decreases with decreasing effective temperature. The position of the maximum of $v_{z, \text { rms }}$ shifts along the model sequence: in the F- and G-star simulations this maximum is almost directly at the optical surface while in the cooler models it is about one to two pressure scale heights below it. In the deeper layers, the convection velocity decreases monotonically with increasing depth in all simulations. In the optically thin upper layers, 

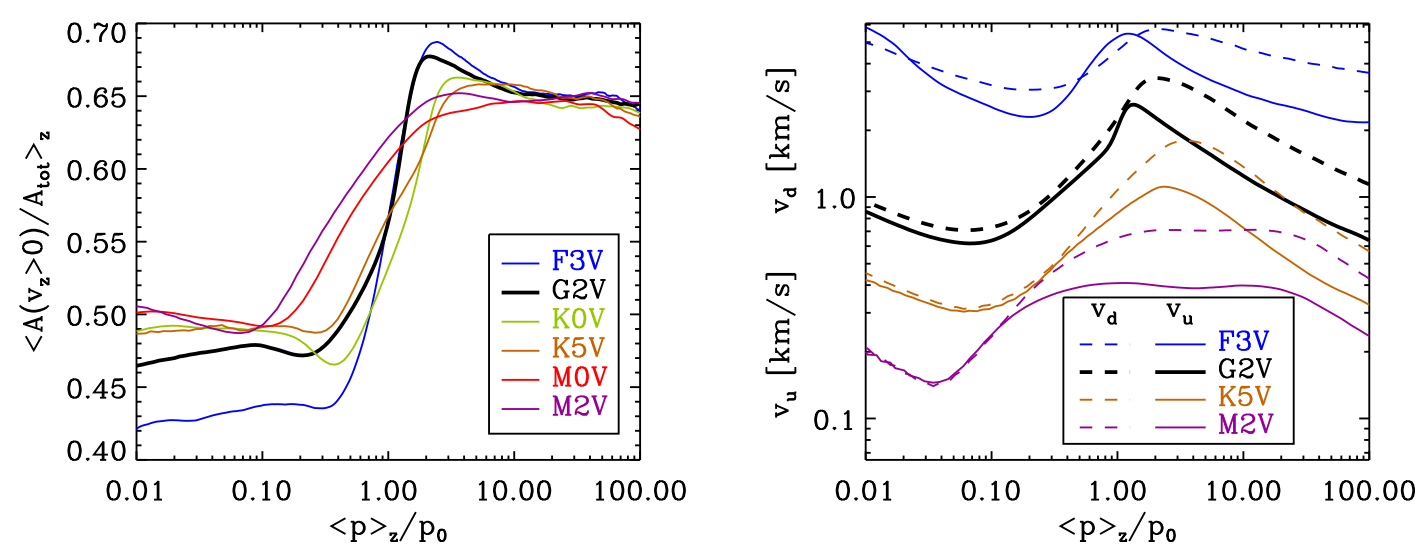

Figure 3.6: Properties of up- and downflows. Left: relative area covered by upflows $\left(v_{z}>0\right)$ on surfaces of constant geometrical depth as functions of normalised averaged pressure. Right: average speed of the upflows, $v_{u}:=\left\langle\left. v_{z}\right|_{v_{z}>0}\right\rangle_{z}$, (solid) and of the downflows, $v_{d}:=\left\langle\left. v_{z}\right|_{v_{z}<0}\right\rangle_{z}$, (dashed) as functions of normalised average pressure for four of the six simulations.

the overshooting large-scale convective motions slow down with increasing height above the optical surface where the stratification is stable against convection. However, the $v_{z, \text { rms }}$ drop only for about one to three scale heights, before they rise again, as shocks become more important.

The right panel of Figure 3.5 shows profiles of the mean Mach number. Although the typical velocities in all simulations reach a substantial fraction of the local sound speed $c_{s}$, only in the atmosphere of the F3V simulation is an average Mach number of order unity reached. Surface convection is largely subsonic in our simulations of M, K, and G stars.

The left panel of Figure 3.6 shows the depth dependence of the relative area of the upflows plotted as functions of normalised average pressure. Below the surface layers, the upflow area is very similar in all six simulations and almost constant at about 63 to $65 \%$ of the total area, which reflects the asymmetry between fast, dense downflows and slower upflows. The value for the upflow area of approximately $2 / 3$ of the total area is in good agreement with the results of Trampedach and Stein (2011) who used another code and different stellar parameters. Near the optical surface, the area fraction of the upflows drops to about $50 \%$ as the strong correlation between vertical velocity and density weakens and the asymmetry between up- and downflows decreases. The low value of the relative upflow area in the upper layers of the F3V simulation of about 42 to $44 \%$ can be interpreted as an effect called reversed granulation in the subadiabatic atmospheric layers (Cheung et al. 2007, and references therein), which inverts the correlation between $v_{z}$ and $\varrho$. This effect is amplified by shock fronts in the F3V simulation: the material trailing the shocks which move upwards is over-dense compared to the average stratification.

The right panel of Figure 3.6 shows the mean speed of the upflows and the downflows. Although both speed profiles peak slightly below the optical surface in all simulated stars, the asymmetry between up- and downflows in the convectively unstable layers leads to 
mean downflow speeds reaching 1.6 to 1.8 times the mean upflow speed in the lower part of the box. In the atmospheres, averaged up- and downflow speeds are almost equal for the four cooler stars. In the F3V simulation, the presence of shocks leads to a steep rise of the average upflow speed in the optically thin layers, whereas the gradient of the averaged downflow speed is flatter (indicating an asymmetry reversed to the one observed below the surface).

\subsubsection{Temperature, pressure, and density}

The upper two panels of Figure 3.7 show the temperature (averaged over iso- $\tau_{R}$ surfaces; for a discussion of the different averages $\langle\cdot\rangle_{\tau},\langle\cdot\rangle_{z}$, and $\langle\cdot\rangle_{p}$, see Appendix A) as function of $\log \tau_{\mathrm{R}}$, both in absolute units and normalised by $T_{\text {eff. }}$. The simulations from F3V to $\mathrm{K} 5 \mathrm{~V}$ show a steep temperature gradient just beneath the optical surface, whereas, in the M dwarfs, the steepest temperature gradient occurs well below this layer (see also Figs 3.9 and 3.10 and Sect. 3.1.4). In the normalised representation, all simulations have a similar profile in the atmosphere $\left(\log \tau_{\mathrm{R}}<0\right)$, while their temperature curves diverge in the subphotospheric layers.

The bottom panel of Figure 3.7 gives the pressure (averaged on iso- $\tau_{\mathrm{R}}$ surfaces) plotted as function of $\log \tau_{\mathrm{R}}$. In the atmosphere, where opacity and temperature are only mildly height-dependent, $\log p$ essentially depends linearly on $\log \tau_{\mathrm{R}}$ as the structure is governed by hydrostatic and radiative equilibrium and is also almost iso-thermal. In the layers just below the photosphere, the curves for the different simulations diverge. The diverging profiles of the subphotospheric temperature (middle panel) and pressure (bottom panel) reflect that the pressure and temperature structures are determined by convection below the photosphere and converge to different adiabat (depending on the stellar parameters) in the deep convective envelopes.

Figure 3.8 shows the depth dependences of the pressure and density scale heights as functions of normalised pressure. We define horizontally averaged local scale heights as

$$
\left\langle H_{p}\right\rangle:=\left(\mathrm{d} \log \langle p\rangle_{z} / \mathrm{d} z\right)^{-1} \text { and }\left\langle H_{\varrho}\right\rangle:=\left(\mathrm{d} \log \langle\varrho\rangle_{z} / \mathrm{d} z\right)^{-1} \text {. }
$$

As the gravitational acceleration increases and the photospheric temperature decreases monotonically from F3V to M2V the, local pressure scale height around $\tau_{\mathrm{R}}=1$ decreases from $\sim 500 \mathrm{~km}$ in $\mathrm{F} 3 \mathrm{~V}$ to $\sim 35 \mathrm{~km}$ in $\mathrm{M} 2 \mathrm{~V}$. In the atmosphere, where the temperature is mildly height-dependent, the local pressure scale height becomes roughly constant. In the convective layers, the strong temperature gradient entails also a strong increase of the pressure scale height towards deeper layers. At $p=100 p_{0}$, near the bottom of the simulation boxes, the local pressure scale height of the F3V simulation is already $\sim 2000 \mathrm{~km}$, which poses a problem for the current implementation of the MURaM code with its fixed vertical cell size (high computational costs).

The peak of the density scale height near the optical surface in some simulations coincides with the strong photospheric temperature gradient of these simulations. Locally, the density scale height often becomes negative at the optical surface in the F3V and G2V simulations (density inversion). In the subsurface layers with high temperature gradient, the density scale heights are somewhat (M2V-G2V:15-30\%, F3V: up to 45\%) larger then the pressure scale heights whereas, in the almost isothermal atmospheres, the scale heights of pressure and density are almost equal. 

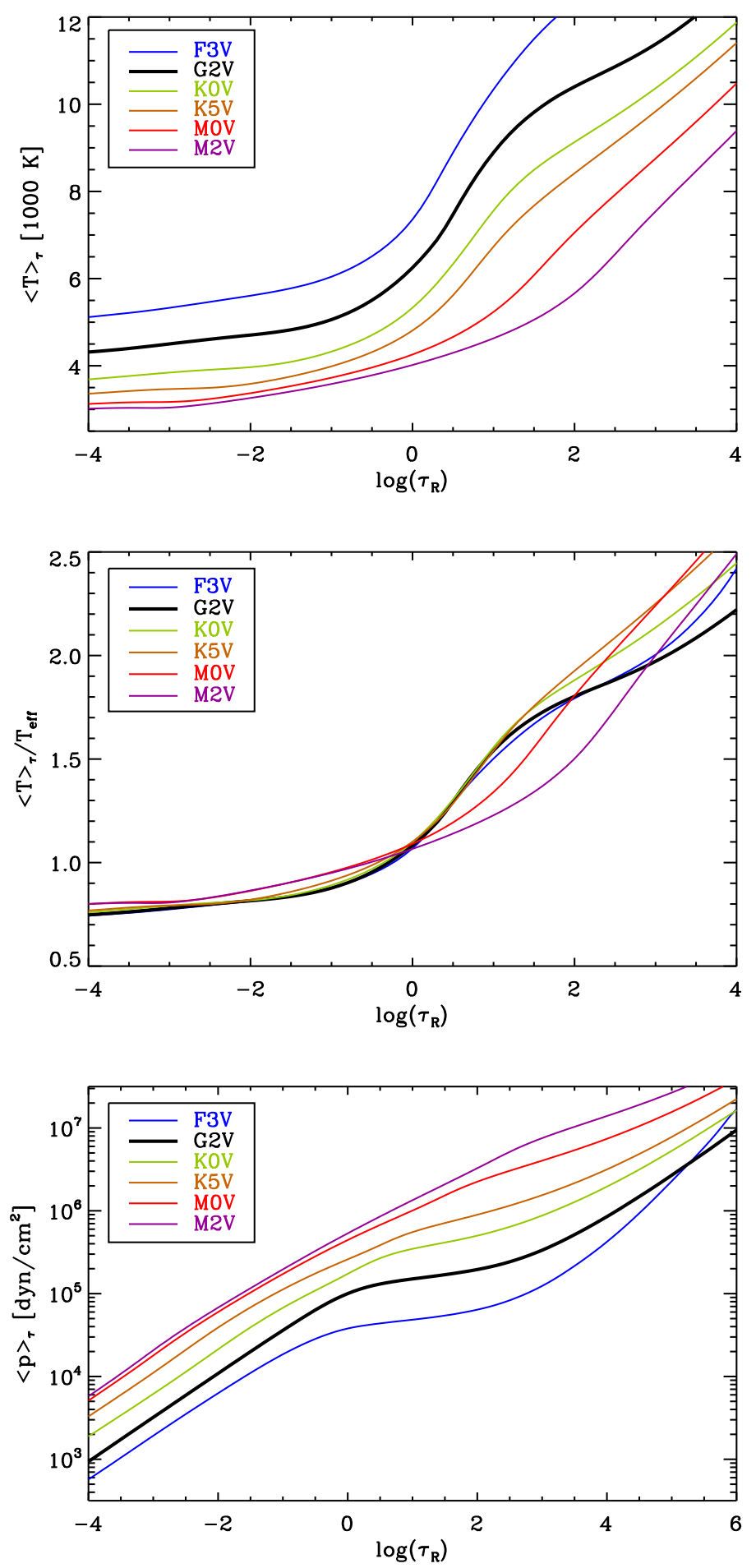

Figure 3.7: Temperature and pressure stratifications on surfaces of constant optical depth. Top panel: temperature averaged over surfaces of constant optical depth. Middle panel: same as top panel, but normalised by the effective temperature of the respective model. Bottom panel: pressure averaged on surfaces of constant optical depth. 

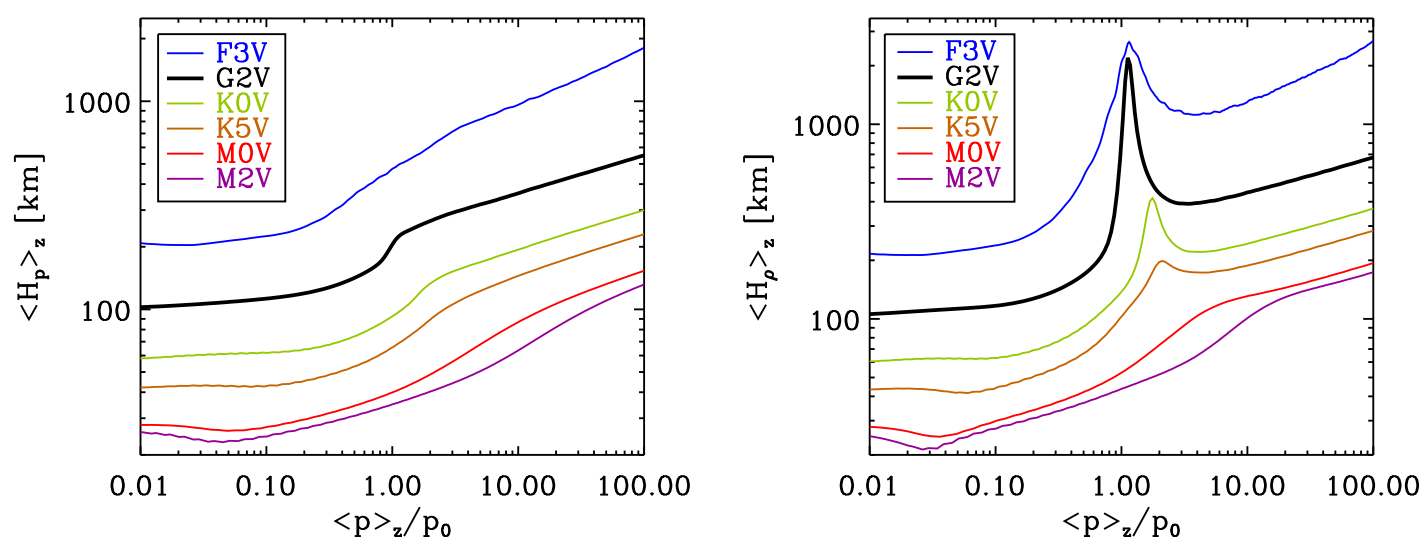

Figure 3.8: Horizontally averaged local scale heights as functions of normalised pressure. Left: pressure scale height. Right: density scale height.
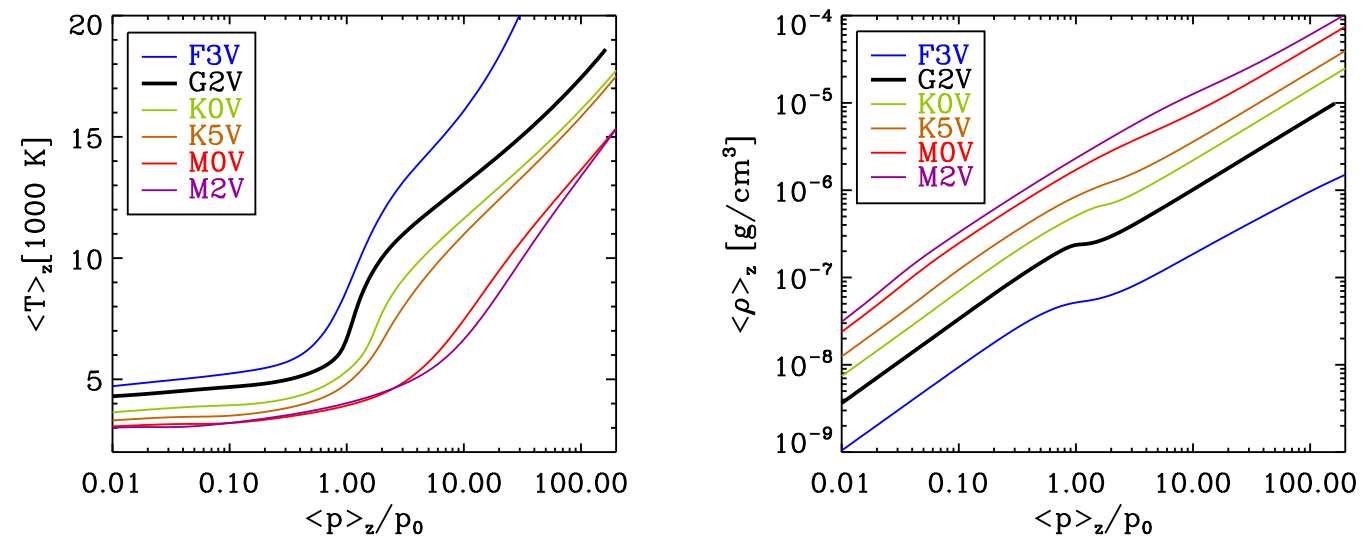

Figure 3.9: Profiles of mean temperature $\langle T\rangle_{z}$ (left panel) and mean density $\langle\varrho\rangle_{z}$ (right panel) as functions of normalised pressure.

Figure 3.9 shows the profiles of temperature and density averaged on iso- $z$ surfaces as functions of the normalised pressure. As already seen in Fig. 3.7 on the $\tau_{R}$ scale, the coolest models lack the strong photospheric temperature gradient of the warmer models. This is shown quantitatively in the left panel of Figure 3.10, which gives the mean profiles of the logarithmic temperature gradient, $\langle\nabla\rangle_{\tau}=\mathrm{d} \log \langle T\rangle_{\tau} / \mathrm{d} \log \langle p\rangle_{\tau}$. Here, the iso- $\tau$ average was chosen because $\nabla$ changes considerably near the optical surface. Since this represents a transition from a (highly) superadiabatic to a subadiabatic regime, averaging over iso-z planes would smear out the sharp photospheric feature in the temperature gradient and thus obscure the relevant physics in this layer.

In the right panel of Figure 3.10, the profile of the superadiabaticity $\langle\nabla\rangle_{\tau}-\left\langle\nabla_{\mathrm{ad}}\right\rangle_{\tau}$ is given. The superadiabaticity in the lowest part of the simulation domain is small $\left(\sim 10^{-3}\right)$ for most of the models, with the exception of the F3V simulation, where the stratifica- 

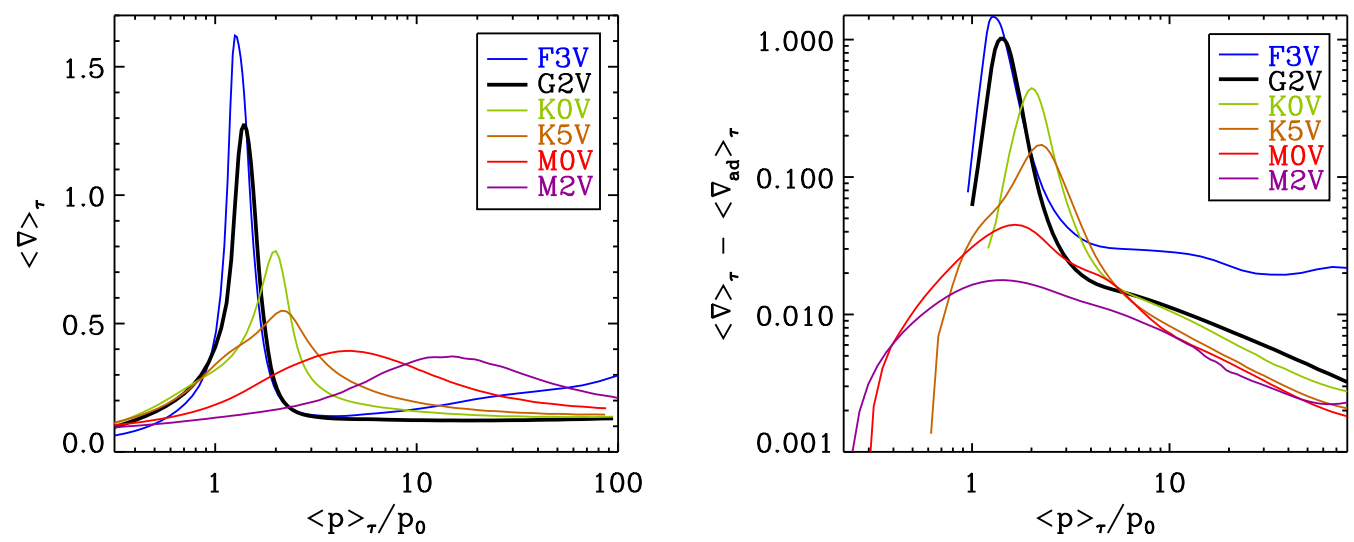

Figure 3.10: Profiles of the logarithmic temperature gradient (left panel) and of superadiabaticity (right panel) averaged on iso- $\tau_{\mathrm{R}}$ surfaces as function of pressure.

tion remains substantially superadiabatic even 5 pressure scale heights below the optical surface. For the M dwarfs, the superadiabaticity is low compared to the hotter models, even in the layers directly beneath the optical surface. This is a consequence of the high densities (i.e. high heat capacity per volume) and low energy fluxes and consequently low horizontal temperature fluctuations.

The top panel of Figure 3.11 shows the relative rms fluctuations of temperature on surfaces of constant optical depth. The relative rms fluctuations of temperature show a monotonic decrease from the hotter to the cooler stars at all depths. This is consistent with the trend in the bolometric intensity contrast (cf. Table 3.1). The subphotospheric peak in the depth profile of temperature fluctuations is at lower optical depth in the Fand G-star simulations compared to the cooler simulations. This has already been pointed out by Nordlund and Dravins (1990a), who coined the expression "hidden" or "veiled" granulation for stars cooler than the Sun, as the maximum temperature contrast occurs far below the optical surface. In the case of the K0V star, the relative temperature contrast at the optical surface is only about $34 \%$ of its peak value at $\log \tau_{\mathrm{R}} \approx 1$ (compared to $42 \%$ in the solar simulation and $64 \%$ in the F3V simulation). The reason for this effect is the lower temperature-sensitivity of opacity near the optical surfaces of the cooler $\mathrm{K}$ and $\mathrm{M}$ stars. This leads to the transition from convective to radiative energy transport occuring at somewhat larger optical depth or normalised pressure (particularly in the K-star simulations) and over a larger optical depth range or normalised pressure range (particularly in the M-star simulations; see Sect. 3.1.4).

The middle panel of Figure 3.11 shows the rms fluctuations of gas pressure on surfaces of constant optical depth. They also diminish with decreasing effective temperature of the simulations, with the notable exception of the G-type star, where the rms fluctuations of pressure on surfaces of constant optical depth are very low in the upper atmosphere compared to the much cooler $\mathrm{K}$ stars. This can be explained as an opacity effect: while the temperature dependence of the Rosseland opacity $\kappa_{\mathrm{R}}(p, T)$ is usually much more important than the pressure dependence, in the temperature range between 4000 and $5000 \mathrm{~K}$ and at pressures between $10^{2}$ and $10^{5} \mathrm{dyn} \mathrm{cm}^{-2}, \kappa_{\mathrm{R}}$ is nearly independent of temperature. 

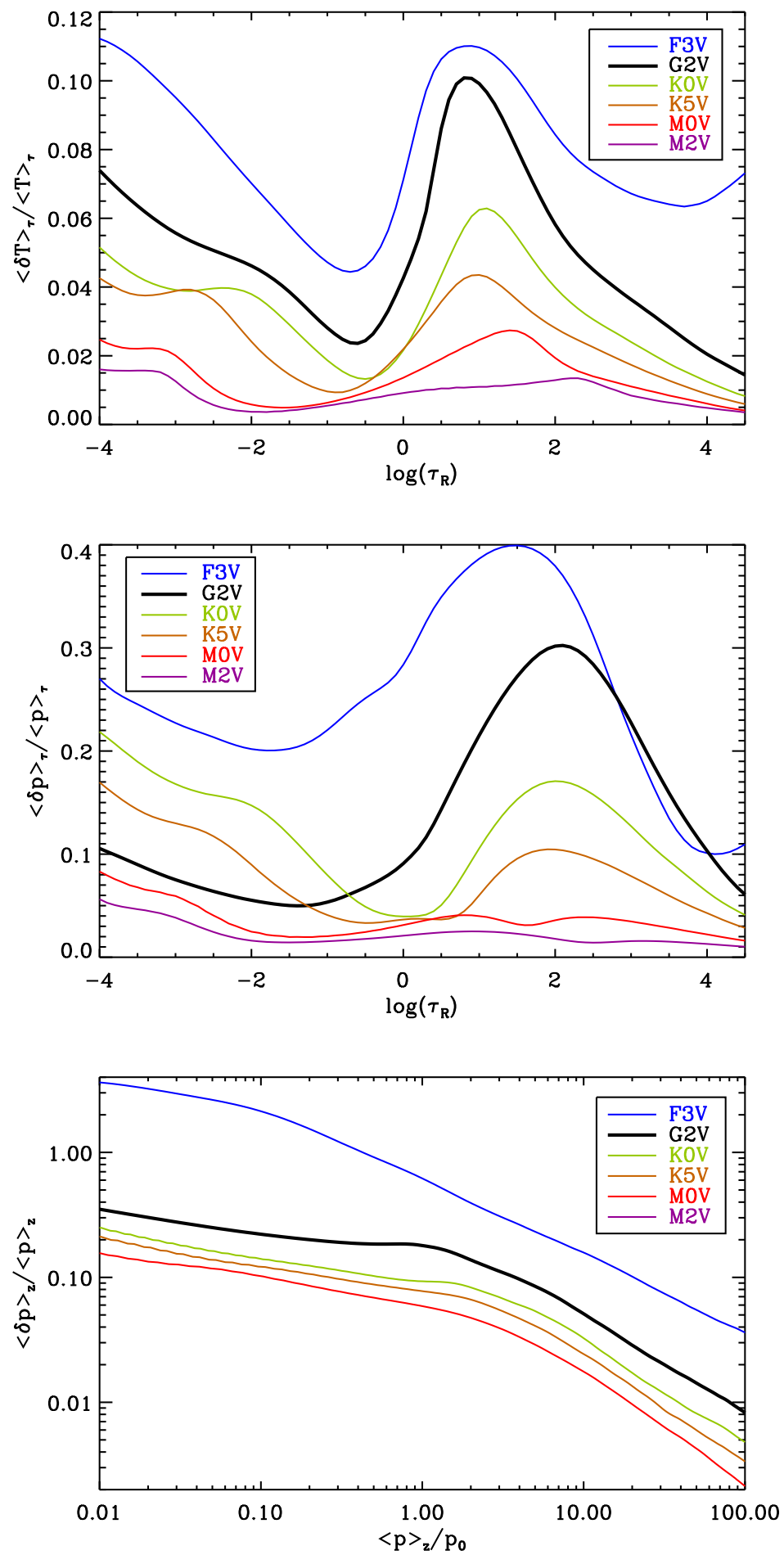

Figure 3.11: Profiles of relative rms fluctuations. Top: temperature fluctuations on surfaces of constant optical depth. Middle: gas pressure fluctuations on surfaces of constant optical depth. Bottom: pressure fluctuations on surfaces of constant geometrical depth. 
Moreover, the temperature fluctuations in the atmosphere are in general relatively small, so that density depends mainly on pressure. Therefore, the increment of the optical depth $\mathrm{d} \tau_{\mathrm{R}}=\left(\kappa_{\mathrm{R}} \varrho\right) \mathrm{d} z$ becomes (almost) independent of temperature, too, so that iso- $\tau_{\mathrm{R}}$ and iso- $p$ surfaces of an atmosphere in this temperature regime are almost identical, i. e. the fluctuations of pressure on surfaces of constant optical depth become small. In our simulation sequence, the G2V star is the only star where the temperature and pressure of the atmospheric layers at $-4 \leq \log \tau_{\mathrm{R}} \leq-1$ fall in the regime of nearly temperature-independent $\kappa_{\mathrm{R}}$.

The bottom panel of Figure 3.11 shows the rms fluctuations of pressure on planes of constant geometrical depth, which are mainly monotonically decreasing with increasing depth and with decreasing effective temperature. This illustrates that in the deeper layers, where velocities and horizontal temperature fluctuations are small, the deviations from hydrostatic equilibrium are small, too. Horizontal temperature fluctuations entail horizontally varying local pressure scale heights: the pressure fluctuations on a horizontal plane in the atmosphere can be regarded as the integrated effect of the temperature fluctuations below this plane.

Before the energy balance of the simulated stellar surface layers is analysed in Sect. 3.1.4, we want to point out that the trends observed in convective velocities and temperature fluctuations can be consistently explained as an effect of the stellar parameters: the lower temperature and higher gravitational acceleration in the atmospheres of cooler stars result in much higher densities. The resulting higher heat capacity per unit volume, $c_{p} / \varrho$, and the much lower net energy flux $\left(F \propto T_{\text {eff }}^{4}\right)$ then have the consequence that the convective motions in the coolest models are less vigorous. This is reflected in the rms velocities of about $0.5 \mathrm{~km} \mathrm{~s}^{-1}$ for those simulations compared to more than $5 \mathrm{~km} \mathrm{~s}^{-1}$ for the F3V-star simulation (see Fig. 3.5). A rough estimate of the convective heat flux $F_{\text {conv }}$ (enthalpy flux) in the spirit of mixing-length theory gives

$$
F_{\mathrm{conv}} \approx \Delta T \cdot v_{\mathrm{conv}} \cdot c_{p} \cdot \varrho,
$$

where $\Delta T$ is the temperature contrast between up and downflows, $v_{\text {conv }}$ is the convective velocity, and $\varrho$ is the density, both of which are assumed to be equal in up- and downflows for this rough estimate. In the convective subsurface layers of cool stars radiative energy transport can be neglected, so that

$$
F_{\mathrm{conv}} \approx F_{\mathrm{tot}}=\sigma T_{\mathrm{eff}}^{4}
$$

If we approximate the quantities $v_{\text {conv }}$ and $\Delta T$ by $\left\langle v_{z, \text { rms }}\right\rangle$ and $\langle\delta T\rangle$, respectively $(\langle\cdot\rangle$ denotes a horizontal and temporal average), and if we further ignore the variation of $c_{p}$ with temperature due to ionisation, we obtain:

$$
T_{\mathrm{eff}}^{4}\langle\varrho\rangle^{-1} \sim\langle\delta T\rangle \cdot\left\langle v_{z, \mathrm{rms}}\right\rangle .
$$

Directly beneath the optical surface, the product on the left-hand side decreases by a factor of about 500 from F3V to M2V, which entails a strong variation of $\langle\delta T\rangle \cdot\left\langle v_{z, \text { rms }}\left(z_{0}\right)\right\rangle$. In fact, the simulations show that both $\langle\delta T\rangle$ and $\left\langle v_{z, \text { rms }}\left(z_{0}\right)\right\rangle$ decrease monotonically through the model sequence by about an order of magnitude each (see Figs 3.4 and 3.11). 

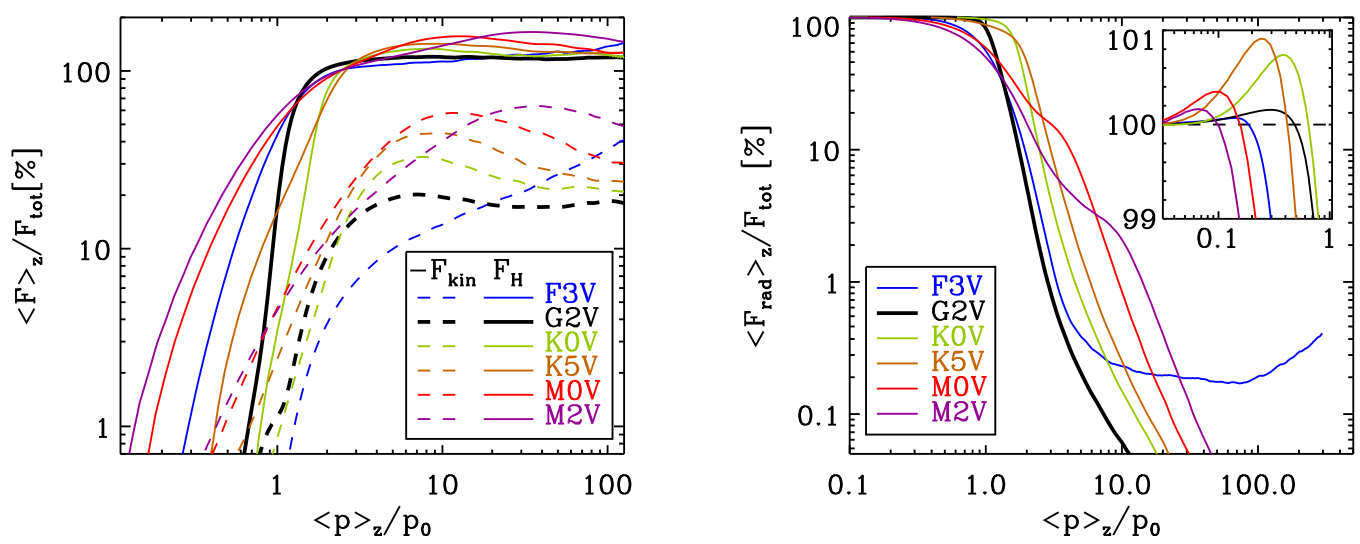

Figure 3.12: Profiles of average energy flux, normalised to total flux. Left: convective energy flux split into net enthalpy flux and net kinetic energy flux. Right: radiative flux.

\subsubsection{Energy flux}

The total energy flux leaving the stellar atmosphere in the form of radiation is supplied by energy flux from below. In the absence of nuclear energy sources, the (temporally averaged) total luminosity is constant throughout the upper layers of a star. In planeparallel geometry, this means that the energy flux is independent of depth.

In the convection zone, the energy flux is almost entirely provided by convective energy transport and is mainly composed of two opposing fluxes: the net enthalpy flux which is directed towards the surface, and the net kinetic energy flux, which is directed inwards. This is a consequence of the asymmetry in temperature and velocity. The fast, cool, and dense downdrafts cary less enthalpy but more kinetic energy (per unit area) than the slow, hot upflows. The left panel of Figure 3.12 shows the profiles of these two fluxes for the six simulated stars, all normalised to the respective total flux. Most models show profiles of the kinetic energy flux levelling off below the optical surface at values between $\sim 20$ to $\sim 60 \%$ of the total flux, larger values corresponding to cooler stars. The F3V model, however, shows a monotonic increase of the kinetic energy flux for increasing depth. The right panel of Figure 3.12 shows the radiative flux in the simulations. The transition from purely convective $\left(F_{\text {rad }} / F_{\text {tot }} \lesssim 0.1\right)$ to mainly radiative energy transport $\left(F_{\text {rad }} / F_{\text {tot }} \gtrsim 0.9\right)$ is quite sharp (within one pressure scale height), except for the M-star simulations, for which this transition takes place over a more extended pressure range. For the K- and M-star simulations, the contribution of the radiative flux to the total flux is larger within the first few pressure scale heights below the surface. One pressure scale height below the surface (i.e. at $p / p_{0}=2.72$ ), in the M0V model, radiation carries already $17 \%$ of the total flux compared to $1.2 \%$ in the $\mathrm{G} 2 \mathrm{~V}$ simulation. Half a pressure scale height below the optical surface $\left(p / p_{0}=1.65\right)$, radiation caries 60 to $70 \%$ of the flux in the K-star simulations, about 25 to $30 \%$ in the M-star models, but only $13.4 \%$ in the simulation of the G2V star. This means that the convective flux directly below the optical surface drops to a relatively small fraction of the total flux for the cooler simulations (especially the $\mathrm{K}$-star simulations). As the convective flux is linked to vertical velocities and temperature 

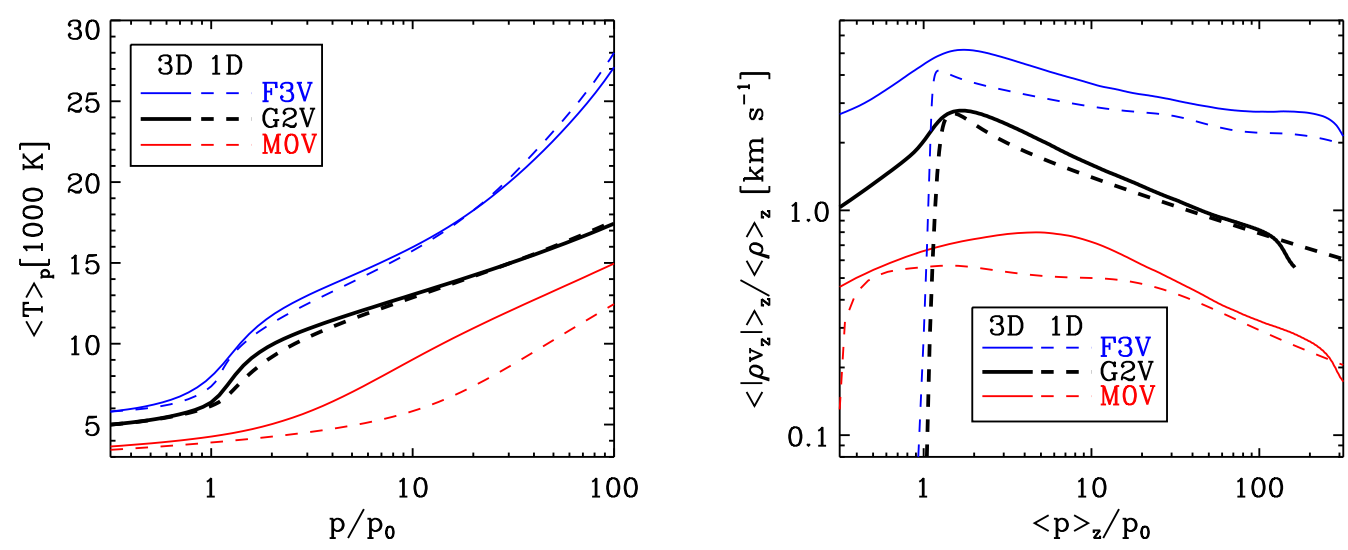

Figure 3.13: Comparison between the 3D MURaM simulations with 1D MLT models. The dashed curves represent results from a 1D MLT model atmosphere and the solid curves represent the averaged simulation results. Left: Profile of temperature (3D: averaged on iso- $p$ surfaces). Right: average vertical velocity, weighted by density (3D: averaged on iso- $z$ surfaces; $1 \mathrm{D}$ : convective velocity). In the $1 \mathrm{D}$ models, the mixing-length parameter $\alpha$ was set to $1.5,1.7$ and 2.0 for F3V, G2V, and M0V, respectively.

contrast between up- and downflows, this is also reflected in the depth-dependence of the rms of the vertical flow velocity (see Fig. 3.5) and the depth profile of the relative rms fluctuations of temperature (see top panel of Fig. 3.11). The peak of the vertical velocity rms is almost exactly at $\tau_{\mathrm{R}}=1$ in the F- and G-star simulations but somewhat deeper in the simulations of cooler stars. Similarly, the peak of the relative temperature fluctuations shifts to higher optical depth from hotter to cooler models. Near the bottom of the simulation box, the contribution of the radiative flux eventually becomes negligible in all simulations except for F3V, where even at this depth radiation still carries about $0.5 \%$ of the energy flux. The much lower density and higher temperature in the subsurface layers of this star enable a somewhat more efficient radiative heat transport.

The inset in the right panel of Figure 3.12 indicates the negative convective energy flux (which follows directly from $\left\langle F_{\text {rad }}\right\rangle_{z}>F_{\text {tot }}$ ) in the convective overshoot region, which is most prominent in the $\mathrm{K}$-dwarf models.

\subsubsection{Comparison to 1D models}

The horizontal averages of our 3D models can be compared to 1D mixing-length models. We used a model grid by Ludwig (priv. comm.) of 1D mixing-length calculations. Analogous to the definition of $\langle z\rangle_{\tau_{\mathrm{R}}=1} \equiv 0$ and $\langle p\rangle_{\tau_{\mathrm{R}}=1} \equiv p_{0}$ as reference points for the $z$ - and $p$-scales in the averaged 3D stratifications, we chose $z\left(\tau_{\mathrm{R}}=1\right) \equiv 0$ and $p\left(\tau_{\mathrm{R}}=1\right) \equiv p_{0}$ as reference points for the 1D models.

Figure 3.13 shows depth profiles of temperature and rms of the vertical velocity as functions of pressure for three of our simulations (F3V, G2V, and M0V) and 1D models with the same $g$ and $T_{\text {eff }}$. The mixing-length parameter was set to $\alpha=1.5,1.7$, and 2.0 for $\mathrm{F} 3 \mathrm{~V}, \mathrm{G} 2 \mathrm{~V}$, and $\mathrm{M} 0 \mathrm{~V}$, respectively. Although the temperature profiles in the almost 
adiabatic sub-surface layers are influenced by the choice of $\alpha$, they could not be brought into exact agreement with the profiles of the 3D results (which, moreover, depend on the averaging method). We therefore chose $\alpha$ close to the values by Trampedach and Stein (2011) for the mass mixing length, which are consistent with the literature values for the MLT- $\alpha$ cited there.

The left panel of Figure 3.13 shows the run of temperature. The $3 \mathrm{D}$ results were averaged on surfaces of constant pressure (as a compromise between the iso- $z$ and iso$\tau_{\mathrm{R}}$ averages, see Appendix A). The general shape of the curves does not differ strongly between averaged 3D and 1D results. However, the temperatures in the 1D models are in general somewhat lower than in the averaged 3D simulations, which can be partly explained by the way in which the $3 \mathrm{D}$ results were horizontally averaged but can also be an effect of the opacity in the optically thin layers, which differs between 1D and our 3D models (the 1D models were calculated with ATLAS6 opacities, see Kurucz 1979). The position and steepness of the strong photospheric gradient does not match the result of the more realistic 3D simulations. This has two reasons: First, this feature is very sensitive to the horizontal averaging method (see Appendix A) because of the strongly corrugated optical surfaces. Second, in this layer overshoot and the transition from convective to radiative energy transport play a major role. The physics behind these effects is essentially three-dimensional and has to be parameterised in a 1D model where only a very crude description of these effects is possible. In the lower part of the depth range considered, there is also a mismatch between the temperature gradients of the averaged 3D and the $1 \mathrm{D}$ results. A disparity in the superadiabaticity (particularly for F3V) and differences in the equation of state between the 3D and 1D models are responsible for this deviation.

In the right panel of Figure 3.13, we show the run of the vertical velocity. For the 3D results, iso- $z$ averages of the density-weighted vertical flow speed are shown. While the gradient of the subsurface velocity and the position of the velocity peak (at least for F3V and $\mathrm{G} 2 \mathrm{~V}$ ) are similar between 1D and 3D models, the 1D models have lower velocities than the 3D simulations (by 10-30\%) in the nearly adiabatic interior. The 1D models obviously lack any velocities in the convectively stable layers, where the simulations display overshooting flows.

In Figure 3.14 the run of the superadiabaticity is shown. The 3D results are shown as iso- $z$ and iso- $\tau_{R}$ horizontal averages. As discussed in Appendix A, near the photospheric transition, the iso- $\tau$ average is closer to the 1D description and therefore more useful for the comparison between 1D and averaged 3D models although the plain horizontal average is the physically more decisive quantity. Despite the necessary parameterisation of important physics in the 1D models, there is a qualitative similarity between 1D and $3 \mathrm{D}$ results. Below its peak, the superadiabaticity of the 1D models is higher by a factor of about 1.5 to 3 compared to the 3D models. For the F3V star, this deviation is the main cause for the difference of the temperature profiles, while for the simulations of the cooler stars, the differences in $\nabla_{\text {ad }}$ related to the equation of state between 1D and 3D models are larger than the small deviation in $\nabla-\nabla_{\mathrm{ad}}$. Around the superadiabatic peak, the profiles of $1 \mathrm{D}$ and 3D results differ more strongly. In this regime, which is more extended in terms of pressure scale heights for the M0V star than for the other two stars, the superadiabaticity in the 3D models is higher than predicted by the 1D models. The superadiabaticity in the 

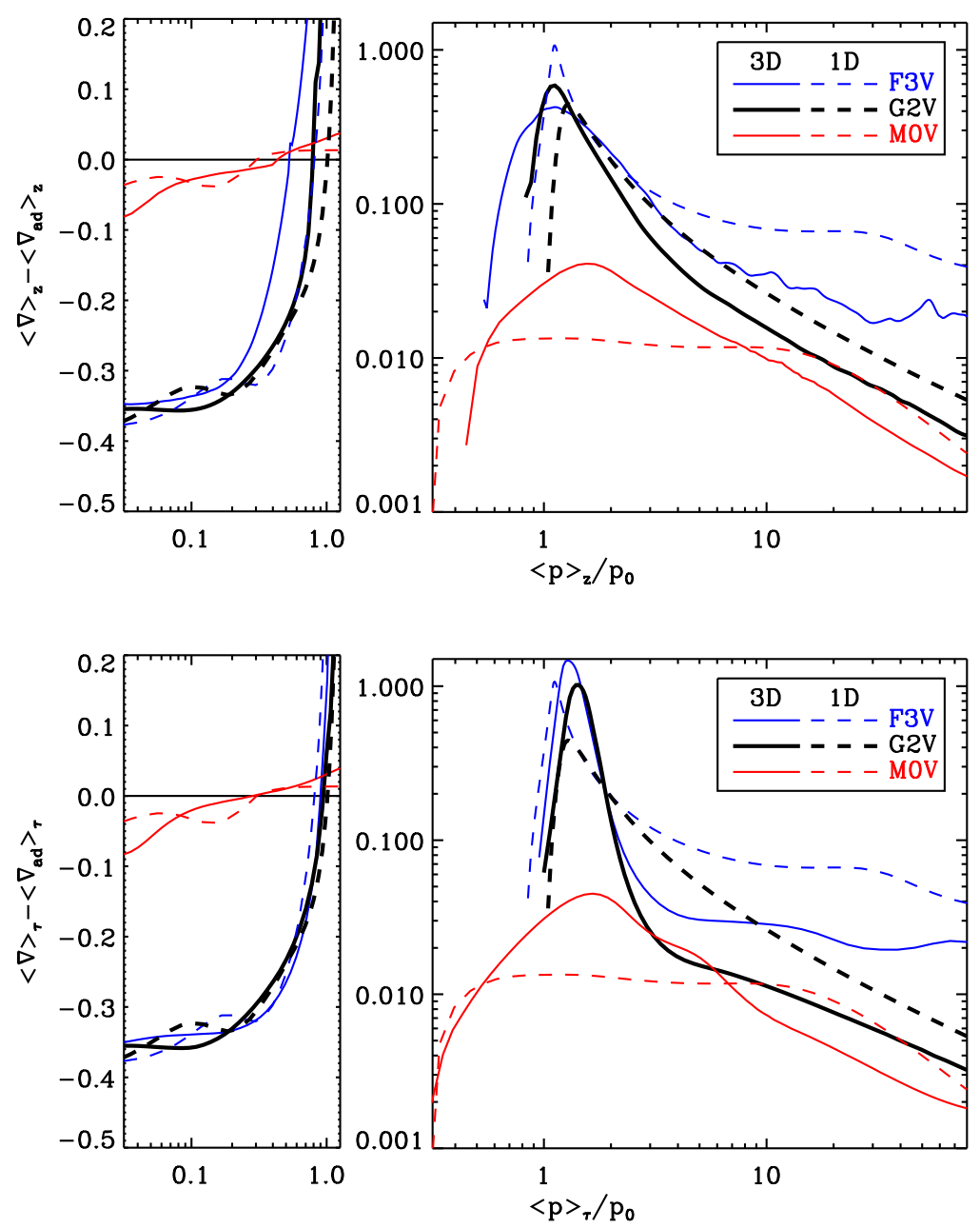

Figure 3.14: Comparison of the superadiabaticity of the 3D MURaM simulations with 1D MLT models. Top: 3D results averaged on planes of constant geometrical depth, $z$. Bottom: $3 \mathrm{D}$ results averaged on iso- $\tau_{\mathrm{R}}$ surfaces. The left sub-plots show the superadiabaticity of the atmospheric layers on a linear scale, the right sub-plots show the superadiabatic regime on a logarithmic scale. In the 1D models, the mixing-length parameter $\alpha$ was set to $1.5,1.7$ and 2.0 for $\mathrm{F} 3 \mathrm{~V}, \mathrm{G} 2 \mathrm{~V}$, and $\mathrm{M} 0 \mathrm{~V}$, respectively.

atmospheric layers is qualitatively in agreement between $1 \mathrm{D}$ and $3 \mathrm{D}$ results. ${ }^{2}$

Although there were small deviations in chemical abundances, equation of state, and opacities between 1D and 3D calculations, most of the differences in the upper part of the depth range shown can be attributed to the necessarily very crude treatment of convection and - most importantly - radiation in the $1 \mathrm{D}$ models versus the comprehensive $3 \mathrm{D}$ simulations.

\footnotetext{
${ }^{2}$ According to R. F. Stein, priv. comm., an analogous comparison of the simulations presented in Trampedach et al. (2013) with 1D MLT models yielded a significantly different result: the superadiabatic peaks are narrower and taller in their 3D simulations compared to the 1D models. This is probably due to the differences in the MLT models used rather than the differences in the 3D simulations.
} 


\subsection{Structure of the magnetic field}

If the magnetic field in a plasma is weak $\left(e_{\text {mag }}=B^{2} /(8 \pi) \ll e_{\text {kin }}\right)$ the Lorentz force acting on the flows does not overcome the inertial forces and the magnetic field has to adjust to the flow patterns. In all simulation runs considered here, the initial magnetic field (homogeneous and vertical) is weak in that sense in the optically thick subsurface layers. Therefore, the convective motions restructure the magnetic field from the arbitrary initial configuration: following the diverging upflows and converging downflows, the magnetic field is compressed in the network of intergranular lanes, while it is diluted in the granules. After this transient phase, a statistically stationary state is reached. As described in Sect. 2.3.3, the analysis in this and later sections is based on six snapshots within this phase for each of the 18 magnetic simulation runs.

Figure 3.15 shows maps of the bolometric intensity in one snapshot each for twelve of the 18 magnetic simulation runs (the remaining six magnetic simulations are shown in Fig. B.1 in Appendix B). ${ }^{3}$ There are significant differences in the visual appearance of the granulation in comparison to the non-magnetic convection (see Fig. 3.1). In all simulations there appear bright structures, which are the stellar analogues of magnetic bright points observed on the Sun (e. g. Keller 1992, Jafarzadeh et al. 2013) and described in MHD simulations of the solar surface convection (e.g. Vögler et al. 2005). In the F3V simulation runs, these brightenings are located in the downflow lanes as well as in their periphery, and sometimes even in larger downflow structures that resemble overbright granules, e.g. in the snapshot of the $500 \mathrm{G}$ run at $(x, y)=(15 \mathrm{Mm}, 2.5 \mathrm{Mm})$. In the cooler models brightenings only occur in the downflow lanes and in the periphery of dark micropores (described below). As displayed in Fig. 3.15 with grey scales saturated at \pm 2.5 standard deviations from the mean, these brightenings become more prominent again from $\mathrm{F} 3 \mathrm{~V}$ to $\mathrm{K} 0 \mathrm{~V}$ and then less prominent from $\mathrm{K} 0 \mathrm{~V}$ to $\mathrm{M} 2 \mathrm{~V}$. This is analysed quantitatively in Sect. 4.3.1.

At high values of $B_{0}$, there also appear dark structures. These are stellar analogues of (micro)pores, which are known from solar observations (e. g. Cho et al. 2010) and have also been studied with MHD simulations of the solar surface convection (e. g. Vögler et al. 2005). For the Sun, there is a critical size of a few hundred kilometers above which magnetic structures become micropores. The sequence of simulations with varying spectral types and $B_{0}$ suggests that this critical size is much smaller in terms of granule sizes for stars cooler than the Sun. Consequently, the value for $B_{0}$ above which micropores are likely to form decreases for cooler stars as less magnetic flux is needed for their formation. While bright structures are still dominant at $500 \mathrm{G}$ in F3V and G2V, dark and bright structures are both prominent in $\mathrm{K} 0 \mathrm{~V}$ and $\mathrm{K} 5 \mathrm{~V}$. In the M-star simulations, dark structures dominate at $500 \mathrm{G}$, in the $\mathrm{M} 2 \mathrm{~V}$ simulation even at $100 \mathrm{G}$. This renders the magnetoconvection in M stars considerably different from the solar example (Beeck et al. 2011). The reasons and consequences of these differences are analysed in more detail in subsequent sections of this thesis. One should, however, take into account that the formation of larger

\footnotetext{
${ }^{3}$ All maps shown in later figures in this section and Sect. 3.3 are taken at the same time step of the simulation runs in order to facilitate the comparison of different quantities at various depth levels.
} 

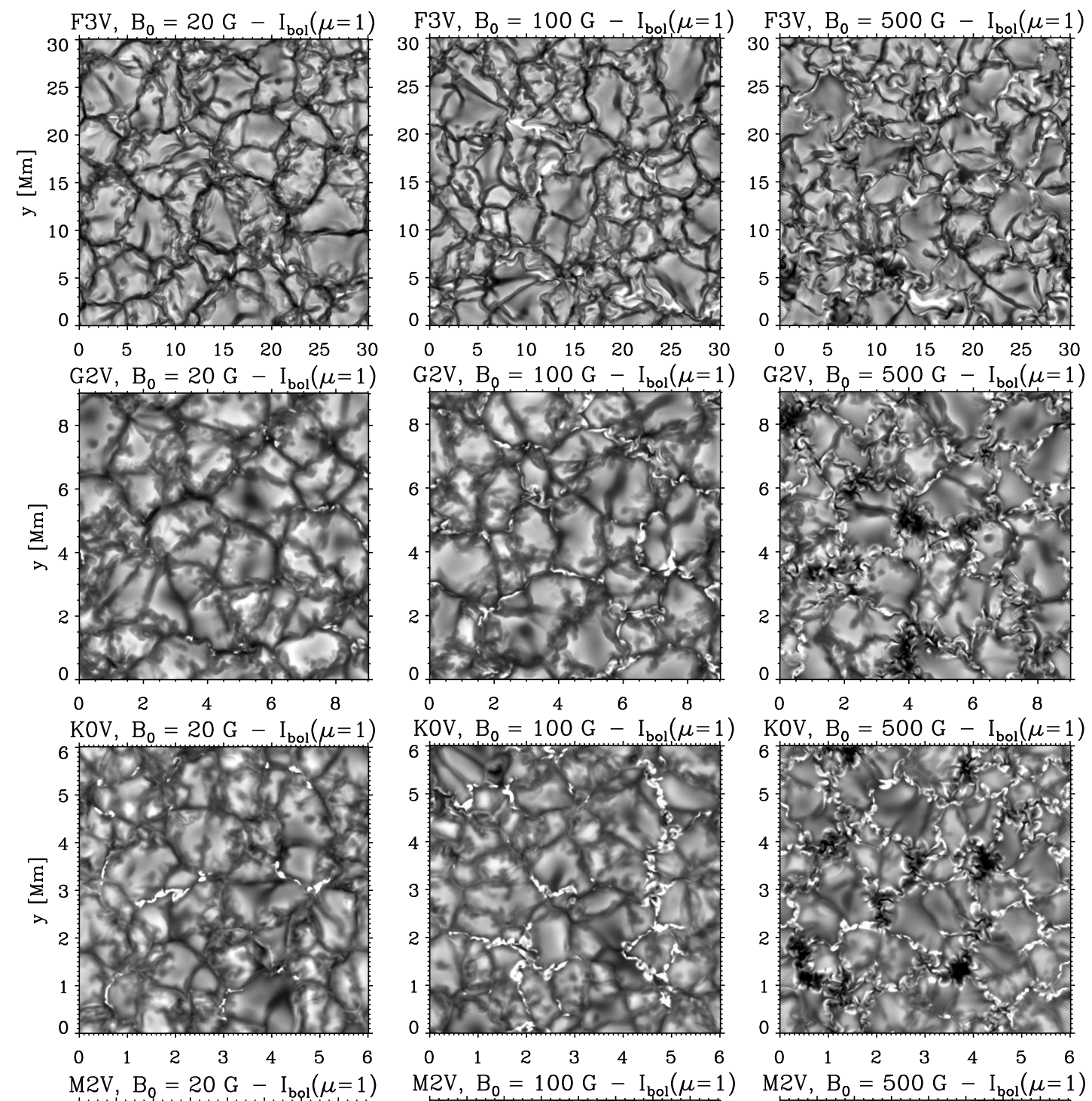

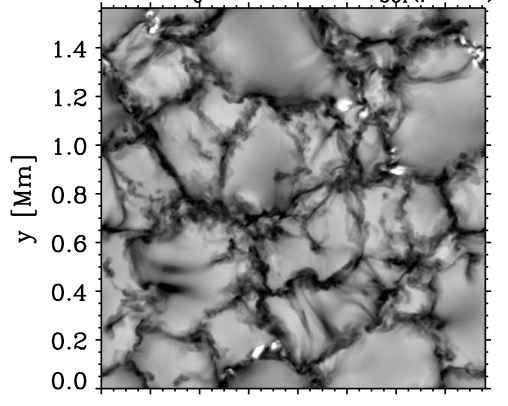

$\begin{array}{llllllll}0.0 & 0.2 & 0.4 & 0.6 & 0.8 & 1.0 & 1.2 & 1.4\end{array}$ $\mathrm{x}[\mathrm{Mm}]$

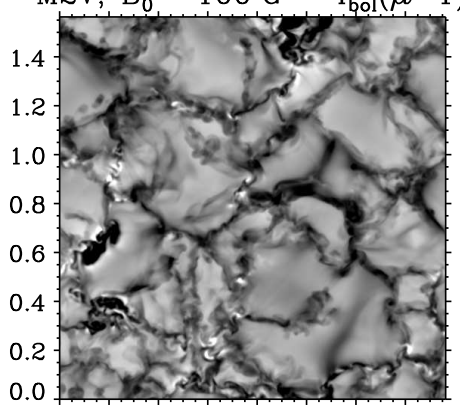

$\begin{array}{lllllllll}0.0 & 0.2 & 0.4 & 0.6 & 0.8 & 1.0 & 1.2 & 1.4\end{array}$ $\mathrm{x}[\mathrm{Mm}]$

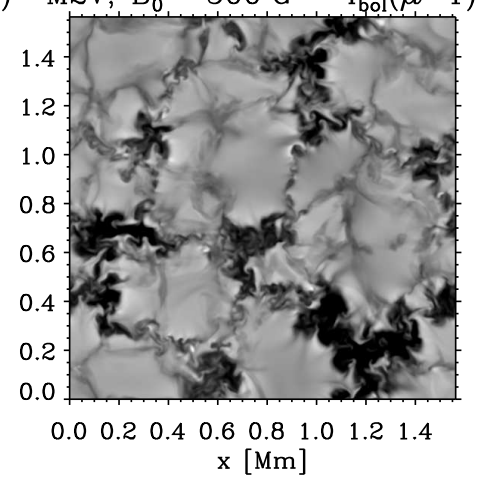

Figure 3.15: Maps of the vertical bolometric intensity for twelve of the 18 magnetic simulation runs (for snapshots of the magnetic K5V and M0V simulations, see Fig. B.1). The grey scale saturates at 2.5 standard deviations below and above the mean intensity. 

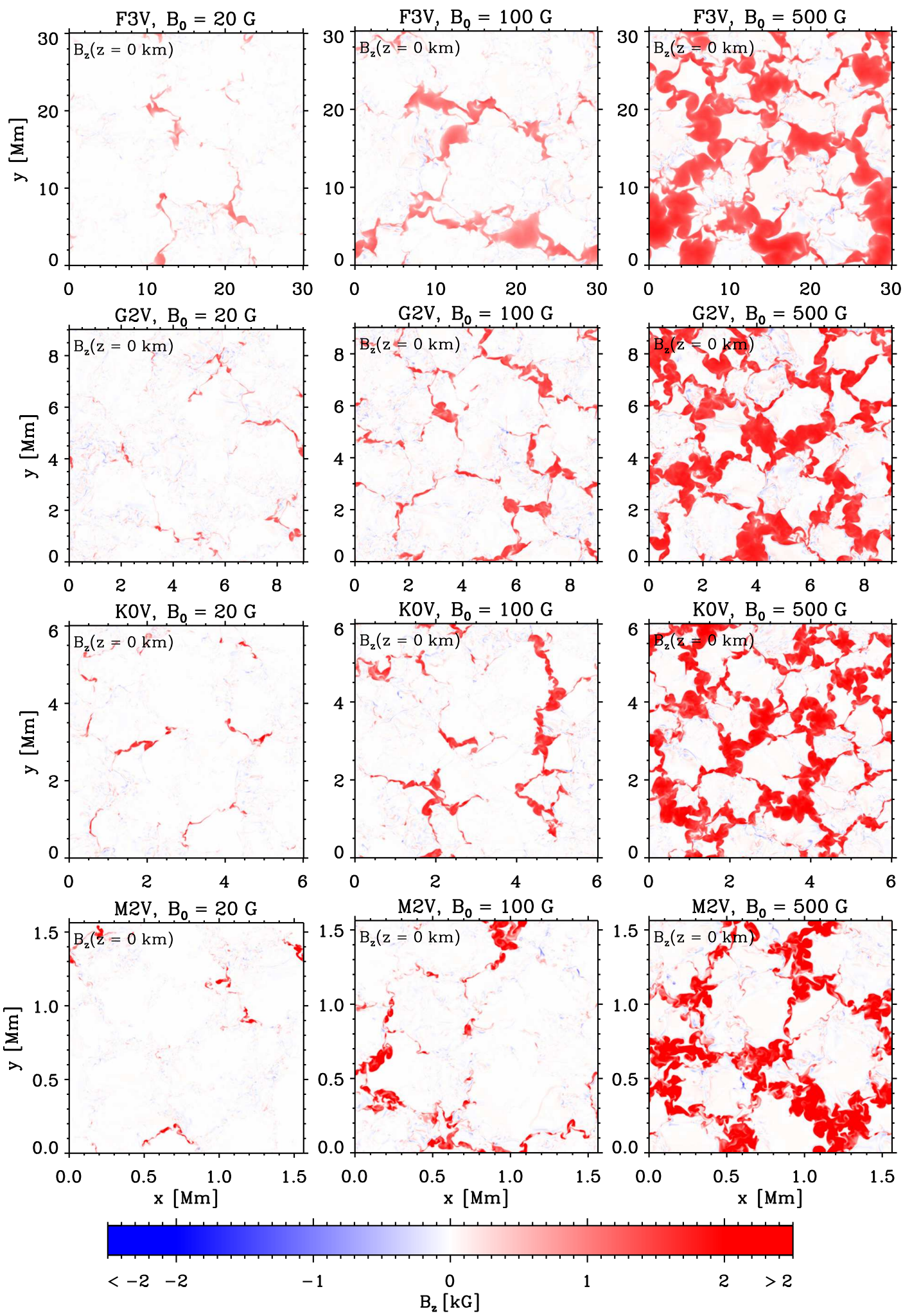

Figure 3.16: Maps of the vertical component $B_{z}$ of the magnetic field strength at the average geometrical depth of the optical surface, i. e. at $z=0$ (same simulation runs and at the same time step as Fig. 3.15; plots for the magnetic K5V and M0V simulations are given in Fig. B.2). 
magnetic structures (such as pores) also depends on the size of the simulation box. In solar simulations with deep and horizontally extended boxes, the formation of micropores and pores occurs also at an averaged field strength of $100 \mathrm{G}$ (Schüssler 2013). To minimise their influence on the results, the simulation boxes considered here were chosen to have similar sizes in terms of pressure scale heights and granule sizes. However, the influence of the limited depth of the simulation boxes needs to be evaluated in future work.

Figure 3.16 shows maps of the vertical component of the magnetic field at the mean level of the optical surface at the same time steps as shown in Fig. 3.15. It is commonly observed in numerical simulations of solar magnetoconvection that the downflow lanes with magnetic flux concentrations form a mesoscale structure (Stein and Nordlund 2000). This is also visible in our stellar simulations: several dark lanes appear in Fig. 3.15, which show a very low magnetic field strength but are evidently downflows (cf. Fig. 3.30). With increasing $B_{0}$, the mesoscale becomes less obvious since more downflow lanes become filled with magnetic flux. This mesoscale is closely related to the deep sub-surface velocity patterns as will be shown in Sect. 3.3.2.

Table 3.2 lists some characteristic values of the magnetic field at the optical surface $\left(\tau_{\mathrm{R}}=1\right)$ for all 18 magnetic simulation runs. The maximum field strength is higher for higher values of $B_{0}$, but the dependence is rather weak, in particular for the models cooler than the Sun. The rms value of the magnetic field at the optical surface depends less than linearly on $B_{0}$ and is almost identical for different stellar types at the same $B_{0}$. The quantities $B_{\text {strong }}, B_{\text {weak }}, B_{\text {eq,kin }}$, and $B_{\text {eq, } p}$ are defined and discussed below.

Figures 3.17 and 3.18 show histograms of the modulus of the magnetic field strength at the optical surface, $\tau_{\mathrm{R}}=1$, and at its average geometrical depth, $z=0$, respectively. The deviation between the distributions based on the two different surfaces is smaller for cooler stars, because they have less corrugated optical surfaces (see Sect. 3.3.1). With logarithmically equidistant bins, the distributions show two peaks, one at a low field strength,

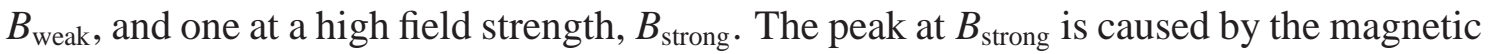
flux concentrations, which show a typical value of the magnetic field (owing to pressure balance as discussed below). As some magnetic field diffuses out of the flux concentrations or is advected back into the upflows by turbulent motions, the magnetic field outside the flux concentrations does not drop to zero but fluctuates around a finite value $B_{\text {weak }}$. $B_{\text {strong }}$ and $B_{\text {weak }}$ both only slightly depend on the stellar type and on $B_{0}$. Values for both peaks of the distributions of $B\left(\tau_{\mathrm{R}}=1\right)$ shown in Fig. 3.17 are given in Table 3.2. The corresponding values for the $B(z=0)$ distribution are very similar for $B_{\text {weak }}$ and somewhat smaller for $B_{\text {strong }}$ (about 150 and $250 \mathrm{G}$ lower in the M- and K-star simulations, respectively, $300 \mathrm{G}$ lower in $\mathrm{G} 2 \mathrm{~V}$ and $500 \mathrm{G}$ lower in $\mathrm{F} 3 \mathrm{~V}$ ). As a consequence of the little variation of $B_{\text {strong }}$ with $B_{0}$ and stellar type, the height of the corresponding peak changes considerably from $B_{0}=100 \mathrm{G}$ to $B_{0}=500 \mathrm{G}$. For $B_{0}=20 \mathrm{G}$ there only is an extended tail of the distribution, but no strong-field peak.

The fact that $B_{\text {strong }}$ is almost independent of $B_{0}$ and of the star is consistent with the rms values of $B$, which change less than linearly with $B_{0}$ and are almost identical for different stars. If small-scale dynamo action is not relevant (because the resolution of the simulations is not sufficient), the main mechanism to enhance the rms of $B$ is the compression of the field in (some) intergranular lanes. In a simple two-component model, one can approximate the magnetic field distribution assuming $B \equiv B_{\text {strong }}$ for the flux concentrations with an area fraction of $f$ and $B \equiv B_{\text {weak }}$ for the remaining area (area 
Table 3.2: Properties of the magnetic field at the $\tau_{\mathrm{R}}=1$ surface (all values in G; errors give temporal 1- $\sigma$ scatter)

\begin{tabular}{lrlccccc}
\hline \hline SpT $^{\mathrm{a}}$ & $\left\langle B_{z}\right\rangle$ & $\max \left(B\left(\tau_{\mathrm{R}}=1\right)\right)$ & $\sqrt{\left\langle B^{2}\right\rangle}$ & $B_{\text {strong }}{ }^{\mathrm{b}}$ & $B_{\text {weak }}{ }^{\mathrm{b}}$ & $B_{\text {eq,kin }}$ & $B_{\text {eq }, p}$ \\
\hline F3V & 20 & $2040 \pm 160$ & $150 \pm 4$ & - & $20 \pm 7$ & $560 \pm 7$ & $989 \pm 6$ \\
F3V & 100 & $4250 \pm 550$ & $445 \pm 4$ & $1418 \pm 67$ & $39 \pm 7$ & $556 \pm 6$ & $994 \pm 4$ \\
F3V & 500 & $5180 \pm 510$ & $1160 \pm 10$ & $1886 \pm 59$ & $77 \pm 9$ & $494 \pm 7$ & $1074 \pm 3$ \\
G2V & 20 & $2310 \pm 110$ & $178 \pm 6$ & - & $47 \pm 3$ & $505 \pm 4$ & $1590 \pm 3$ \\
G2V & 100 & $3250 \pm 410$ & $412 \pm 10$ & $1702 \pm 74$ & $62 \pm 4$ & $492 \pm 6$ & $1598 \pm 7$ \\
G2V & 500 & $3550 \pm 150$ & $1022 \pm 11$ & $1990 \pm 18$ & $79 \pm 10$ & $444 \pm 6$ & $1681 \pm 9$ \\
K0V & 20 & $2890 \pm 160$ & $175 \pm 7$ & - & $27 \pm 4$ & $471 \pm 3$ & $2088 \pm 3$ \\
K0V & 100 & $2930 \pm 130$ & $425 \pm 3$ & $1824 \pm 24$ & $37 \pm 5$ & $450 \pm 6$ & $2094 \pm 2$ \\
K0V & 500 & $3390 \pm 140$ & $1031 \pm 6$ & $2056 \pm 11$ & $44 \pm 3$ & $381 \pm 6$ & $2158 \pm 2$ \\
K5V & 20 & $2640 \pm 170$ & $176 \pm 4$ & - & $26 \pm 5$ & $509 \pm 10$ & $2548 \pm 2$ \\
K5V & 100 & $2980 \pm 80$ & $418 \pm 6$ & $1823 \pm 48$ & $14 \pm 2$ & $463 \pm 9$ & $2560 \pm 3$ \\
K5V & 500 & $3550 \pm 140$ & $1043 \pm 3$ & $2099 \pm 13$ & $52 \pm 3$ & $404 \pm 5$ & $2614 \pm 4$ \\
M0V & 20 & $3400 \pm 240$ & $185 \pm 5$ & - & $27 \pm 3$ & $494 \pm 13$ & $3341 \pm 3$ \\
M0V & 100 & $3900 \pm 200$ & $436 \pm 4$ & $1917 \pm 41$ & $23 \pm 5$ & $465 \pm 13$ & $3351 \pm 2$ \\
M0V & 500 & $4200 \pm 200$ & $1075 \pm 3$ & $2326 \pm 15$ & $52 \pm 3$ & $375 \pm 3$ & $3433 \pm 2$ \\
M2V & 20 & $3500 \pm 580$ & $182 \pm 3$ & - & $34 \pm 6$ & $426 \pm 5$ & $3666 \pm 3$ \\
M2V & 100 & $4090 \pm 370$ & $440 \pm 8$ & $1994 \pm 85$ & $34 \pm 8$ & $406 \pm 7$ & $3675 \pm 2$ \\
M2V & 500 & $4450 \pm 320$ & $1067 \pm 7$ & $2352 \pm 57$ & $40 \pm 4$ & $322 \pm 2$ & $3758 \pm 2$
\end{tabular}

a spectral type of the simulation

${ }^{b}$ peaks of the histograms of Fig. 3.17 

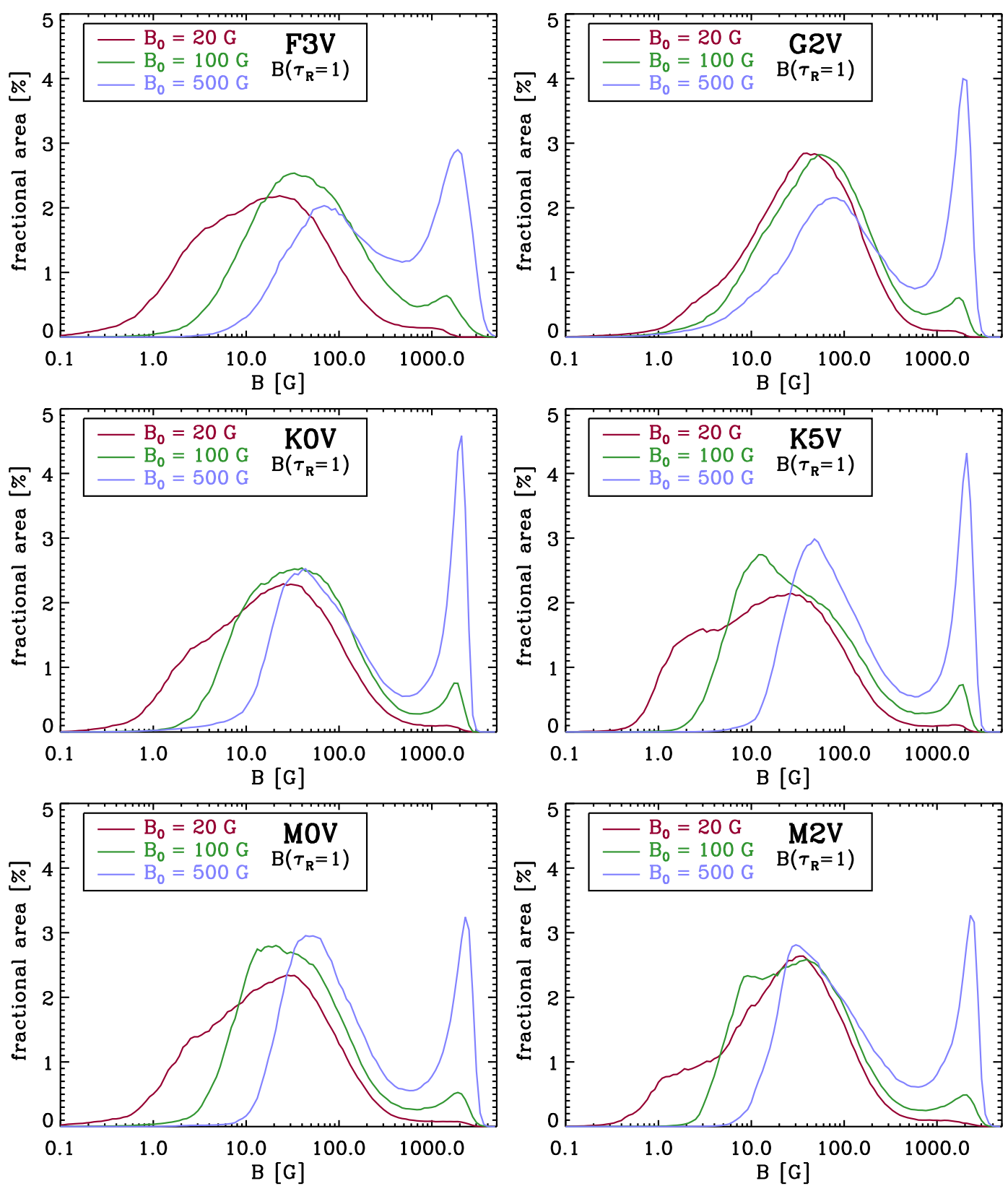

Figure 3.17: Histograms of the modulus $B$ of the magnetic field strength at the optical surface, $\tau_{\mathrm{R}}=1$.

fraction of $1-f)$. With the further simplifying assumption $B_{\text {weak }}=0$, this yields $f=$ $B_{0} / B_{\text {strong }}$ and the rms is then $\left\langle B^{2}\right\rangle^{1 / 2}=B_{\text {strong }} f^{1 / 2}=\left(B_{\text {strong }} B_{0}\right)^{1 / 2}$. With $B_{\text {strong }}=2000 \mathrm{G}$, this simple model predicts rms values of the magnetic field of 200,447 , and $1000 \mathrm{G}$ for $B_{0}=20,100$, and $500 \mathrm{G}$, respectively, which are very close to the actual values given in Table 3.2 .

An interesting fact is, that the area with $B\left(\tau_{\mathrm{R}}\right)<0.1 \mathrm{G}$ is a negligible part of the optical surface. Even at $B_{0}=20 \mathrm{G}$, there is an ubiquitous magnetic field of a several $\mathrm{G}$, which is consistent with solar observations employing the Hanle effect (Trujillo Bueno 

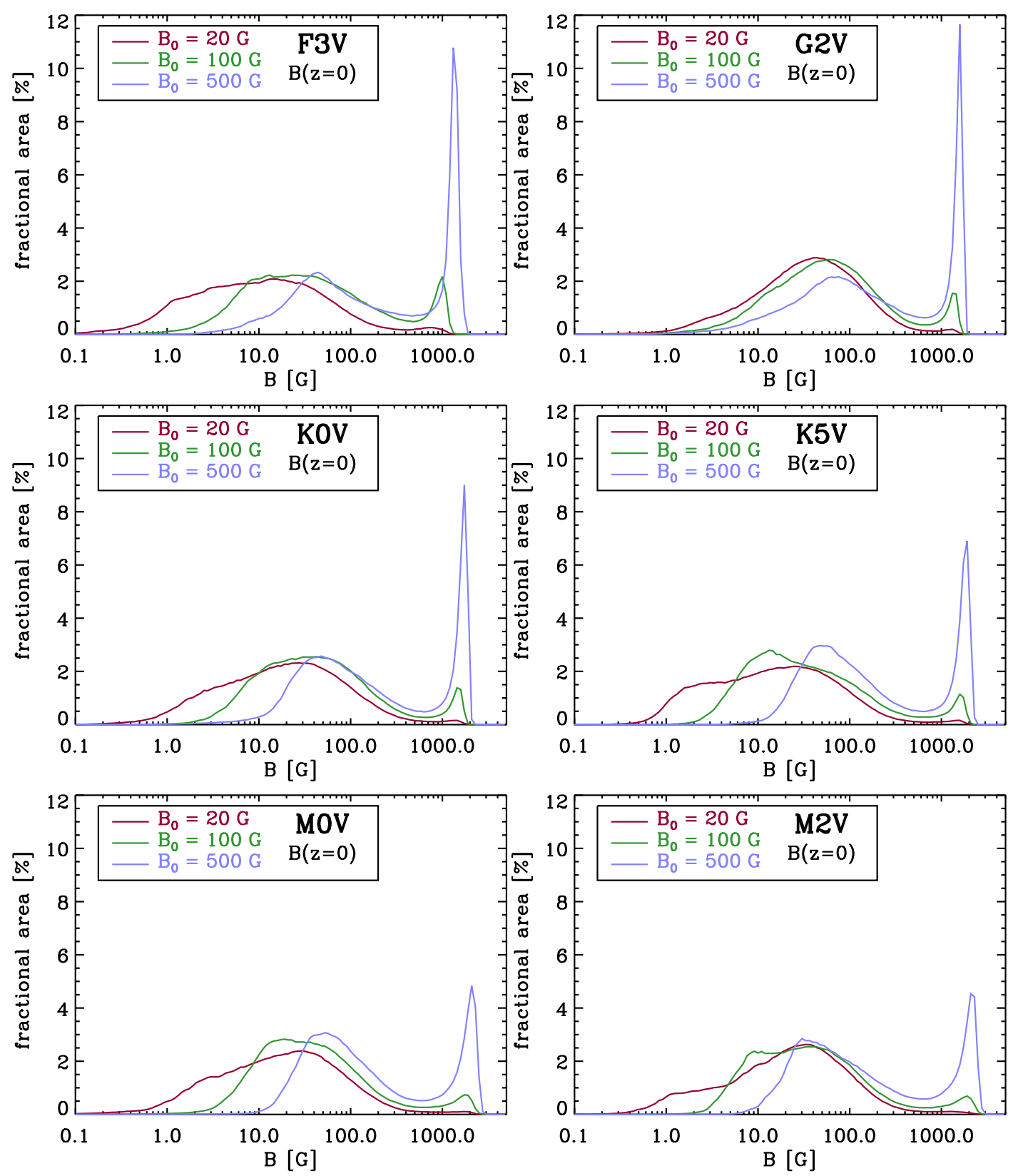

Figure 3.18: Histograms of the modulus $B$ of the magnetic field strength at the geometrical depth $z=0$, i.e. at the mean level of the optical surface.

et al. 2004, Stenflo et al. 2002). Most Hanle-effect measurements, however, indicate a somewhat higher magnetic field strength of $\sim 100 \mathrm{G}$. This apparent discrepancy is probably due to the finite spatial resolution of the simulations: the field found by the Hanle-effect measurements is probably very turbulent and fluctuates on length scales far below $10 \mathrm{~km}$, which are not resolved by the MHD simulations.

Figure 3.19 shows joint histograms of the modulus of the magnetic field and its angle with respect to the surface in the near-surface layers for several simulations. In all cases, a weak-field and a strong-field component can be distinguished. While weak fields occur at 

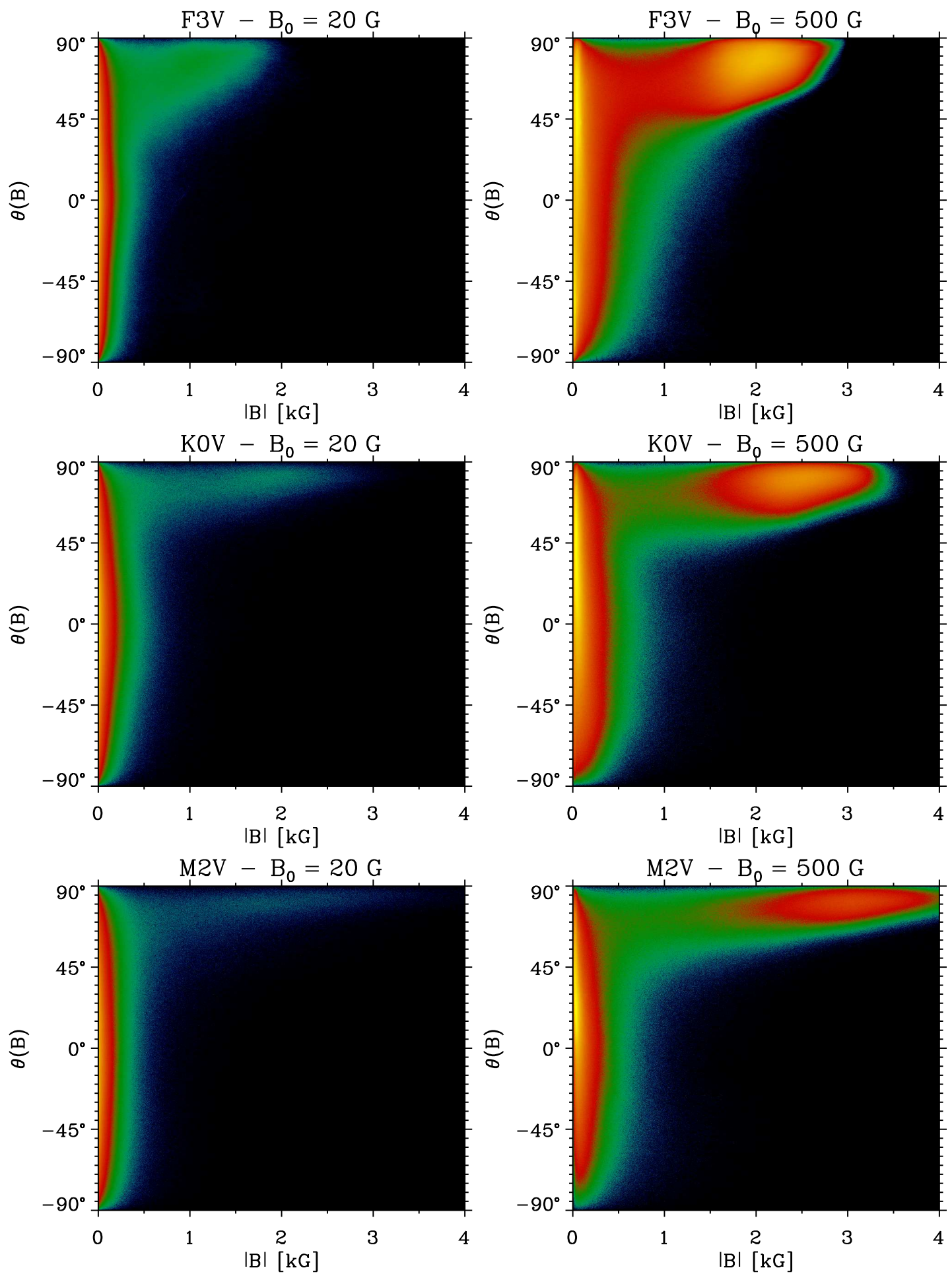

Figure 3.19: Joint histograms of the modulus of the magnetic field and its angle $\theta(\mathbf{B})$ with respect to the surface in a layer between $\left\langle z\left(p=p_{0} \exp (-0.5)\right)\right\rangle$ and $\left\langle z\left(p=p_{0} \exp (+0.5)\right)\right\rangle$, where $p_{0}=\left\langle p\left(\tau_{\mathrm{R}}=1\right)\right\rangle$ is the mean pressure at the optical surface. Red and yellow indicate high point density; black, blue and green regions indicate low point density. 
nearly arbitrary inclination, the stronger fields show a strong preference for near vertical directions. This is not restricted to simulations with high values of $B_{0}$ : even at $B_{0}=$ $20 \mathrm{G}$ all points with a field of more than about $1 \mathrm{kG}$ have an inclination of the field of $\theta(B) \gtrsim 45^{\circ}$. The only apparent differences between the stars of different spectral types are that the field modulus of the strong field regime becomes larger and the distribution of angles around vertical orientation becomes narrower at lower effective temperature. The distribution of magnetic field directions explains why Stokes-V measurements do not detect the few-G volume filling field in the Sun: positive and negative inclinations cancel each other within one resolution element of the measurements (Stenflo et al. 2002).

As the magnetic field is locally enhanced by the interaction with the flows, its pressure can locally dominate the turbulent pressure and the gas pressure. In these regions, the flows are strongly influenced by the magnetic field. The Lorentz force overcomes the inertial forces if the magnetic energy density surpasses the kinetic energy density, which is the case if the field strength exceeds the equipartition field strength

$$
B_{\text {eq,kin }}:=\sqrt{4 \pi \rho v^{2}} .
$$

In Table 3.2 values for this equipartition field strength are given on the basis the average kinetic energy density at the optical surface. In all cases, the magnetic flux concentrations are far above equipartition. If equipartition with the kinetic energy density at the optical surface is exceeded, the outflows from the granules cannot penetrate the flux concentrations anymore. As the surface layers of cool stars are convectively unstable, the plasma in the flux concentration sinks down, which causes the structure to horizontally contract. As the field is amplified by this contraction the magnetic pressure inside the flux concentrations rises until the sum of gas and magnetic pressure inside the structure are in equilibrium with the external gas pressure. This mechanisms can produce flux concentrations with magnetic fields far above the equipartition field strength $B_{\text {eq,kin. }}$.

As it is important for the dynamics and structure of the magnetic field one often distinguishes between the cases of low and high plasma- $\beta$,

$$
\beta:=\frac{8 \pi p}{B^{2}},
$$

where $p$ is the gas pressure. Low- $\beta$ regions are dominated by the magnetic pressure and the Lorentz force cannot be balanced by gas pressure gradients (i.e. the field has to adjust to a force-free configuration), while high- $\beta$ regions are dominated by the gas pressure, which allows the existence of pressure-balanced flux tubes. The corresponding equipartition field strength (at which $\beta=1$ ) is

$$
B_{\text {eq, } p}:=\sqrt{8 \pi p}
$$

Within our model sequence, the surface pressure increases from F3V to M2V. Thus, the average $B_{\text {eq, } p}$ increases from about $1 \mathrm{kG}$ at the optical surface of the $\mathrm{F} 3 \mathrm{~V}$ star to $3.7 \mathrm{kG}$ at the optical surface of the M2V simulation (values based on the average gas pressure at the optical surface, see Table 3.2). As the optical surfaces (especially of the F- and Gstar simulations) are strongly corrugated, the more relevant quantity is the field strength at which the gas pressure outside a flux concentration is equal to the magnetic pressure inside at the same geometrical depth (e.g. at the depth where the optical surface in the 

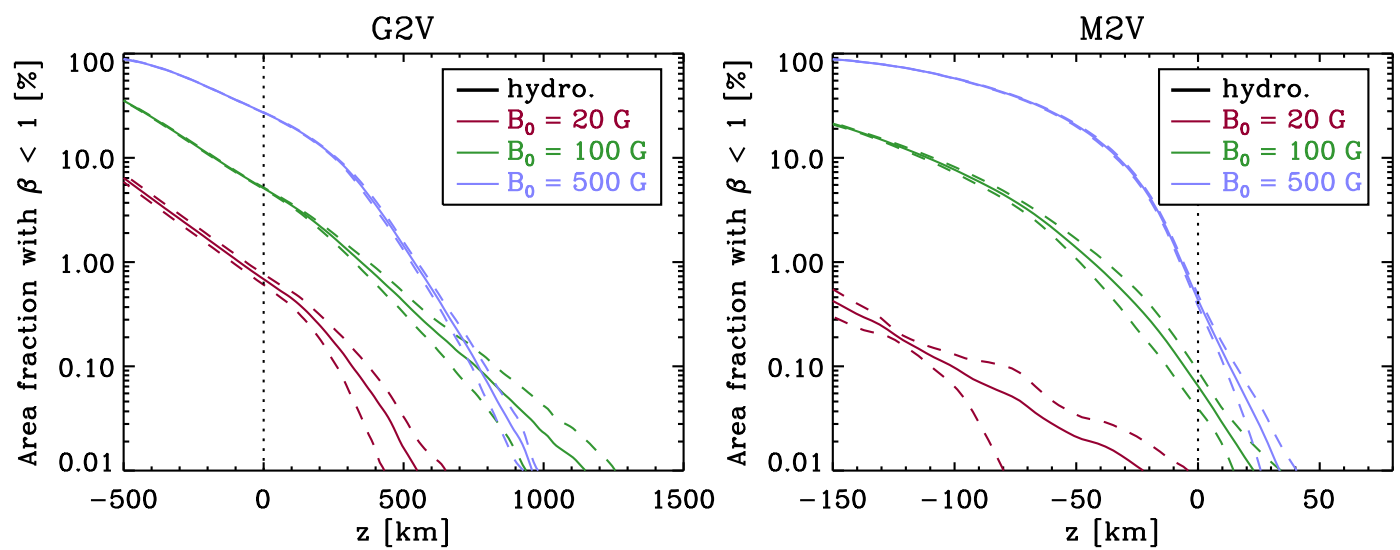

Figure 3.20: Area fraction of low- $\beta$ regions $(\beta<1)$, i. e. regions dominated by magnetic pressure, in the G2V (left) and $\mathrm{M} 2 \mathrm{~V}$ (right) simulations.

magnetic flux concentration is located). This value is considerably higher because the optical surface is locally depressed in the flux concentrations. As is explained in more detail in Sect. 3.3.1, this depression is caused by the strong reduction of the gas pressure inside the flux concentrations. The field strength $B_{\text {strong }}$ is close to equipartition with the external pressure in F- and G-type stars at the optical surface of the flux concentrations. This means, that the flux tubes are mostly evacuated and a higher compression of the field is no longer possible. In cooler stars, the equipartition with the external gas pressure is not reached. This might be a result of radial radiative heating of the magnetic flux concentrations. The radiative cooling time scales at the optical surface of stars cooler than the Sun are larger because the temperature is lower and the density (and thus the heat capacity per volume) is higher. As shown in Sect. 3.1.4, radiative energy transport is more important in the layers directly below the surface in $\mathrm{K}$ and $\mathrm{M}$ stars than it is in $\mathrm{F}$ and G stars because the absorption coefficient is less sensitive to temperature around 4000$5000 \mathrm{~K}$ than it is at higher temperatures. The flux concentrations can thus be radially heated in the layers directly below the surface on a time scale similar to the radiative cooling time scale of the structure. A more detailed analysis of the convective collapse of the tubes is beyond the scope of this work.

Figure 3.20 shows the height-dependence of the area fraction of low- $\beta$ regions (i.e. regions with $\beta<1$ ) for the $\mathrm{G} 2 \mathrm{~V}$ and $\mathrm{M} 2 \mathrm{~V}$ simulations. The low- $\beta$ regions have a much lower area fraction at the optical surface of the M2V star for all values of $B_{0}$. With increasing height, the gas pressure drops roughly exponentially, while the horizontally averaged magnetic pressure has a lower limit of $\left\langle B_{z}\right\rangle^{2} /(8 \pi)=B_{0} /(8 \pi)$ within the simulated unipolar regions. Therefore, the upper photospheric layers are mostly dominated by the magnetic field (i.e. $\beta<1$ ) in our simulations, especially at $B_{0}=500 \mathrm{G}$.

Figure 3.21 shows maps of the vertical component of the magnetic field at a height corresponding to 4.6 pressure scale heights above the mean level of the optical surface. At this height, the magnetic pressure is on average far higher than the gas pressure. Consequently, the magnetic field is much less structured by the flows and is less concentrated in the downflows. Even at $B_{0}=500 \mathrm{G}$, there are, however, some small almost field-free regions at this height, which are situated above the centres of mesogranules. Although 

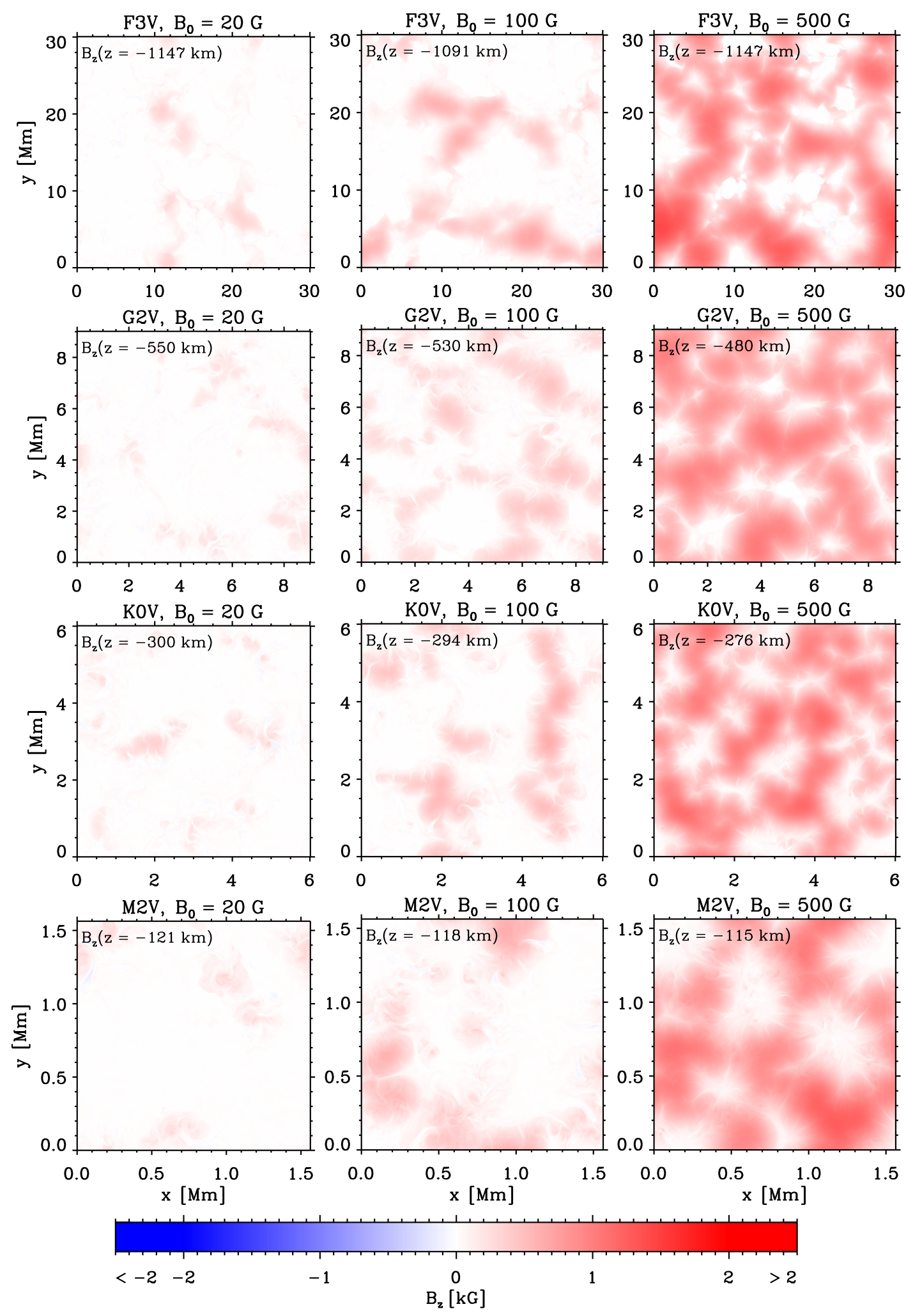

Figure 3.21: Maps of the vertical magnetic field component at a height corresponding to 4.6 pressure scale heights above the mean level of the optical surface. The geometrical depth $z$ of this level is specified in each panel. Plots for the K5V and M0V simulations are given in Fig. B.3. 

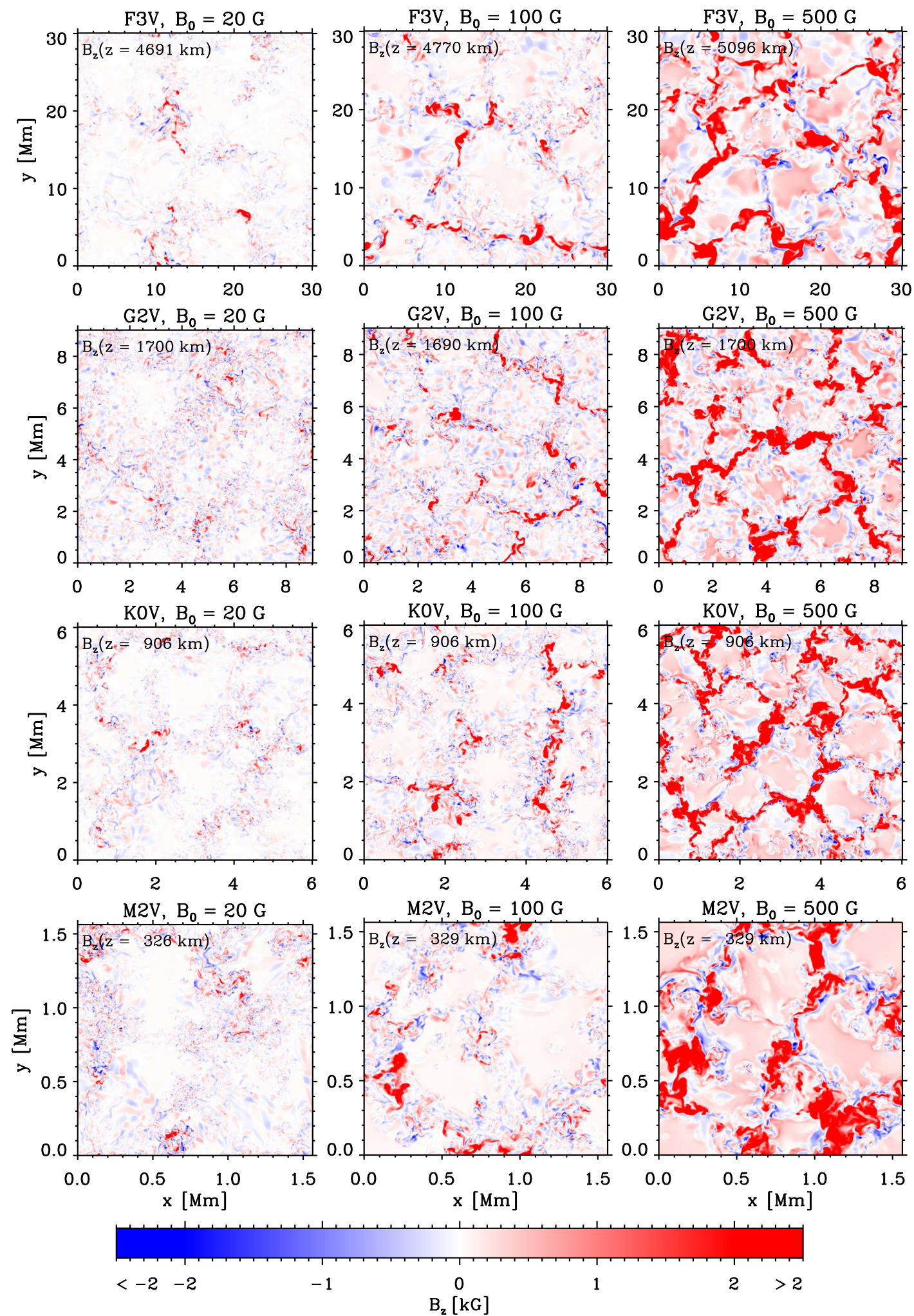

Figure 3.22: Same as Fig. 3.21, but 4.6 pressure scale heights below the mean level of the optical surface. Plots for the K5V and M0V simulations are given in Fig. B.4. 
the magnetic field seems rather smooth in this layer, it is capable of imposing a complex structure on the flows as will be analysed in Sect. 3.3.2.

The higher layers, where the magnetic field determines the dynamics, do not play a significant role for the convective energy transport of the star as they are optically thin and convectively stable. However, these layers are relevant for the formation of spectral lines, which are used to infer observational information on the magnetic field of stars.

Figure 3.22 shows the vertical component of the magnetic field 4.6 pressure scale heights below the average level of the optical surface. The pattern in this depth looks roughly similar to the pattern at the optical surface shown in Fig. 3.16. In addition, there are patches of inversed polarity in the shear layers between up- and downflows where the fluid turns over. In these layers, some overturning flows penetrate the flux concentrations somewhat and twist the magnetic field lines downwards, which causes inversed polarity patches around the flux concentrations. In the downflows, the magnetic field strength is locally somewhat higher than at the surface (up to $9.2 \mathrm{kG}$ in the K0V simulation) due to the strong compression in the converging downflows. Here, the magnetic field is of the same order of the local value of $B_{\text {eq,kin }}$, but small compared to $B_{\text {eq, } p}$.

\subsection{Effects of the magnetic field on the atmospheric struc- ture}

\subsubsection{Optical depth structure}

The opacity, $\kappa$, in a solar-metallicity star at temperatures between $\sim 4000 \mathrm{~K}$ and $\sim 7000 \mathrm{~K}$ can be mainly attributed to bound-free transitions of the $\mathrm{H}^{-}$ion (see, e. g., Gray 2005, and references therein). In this temperature regime, the abundance of $\mathrm{H}^{-}$is roughly proportional to the electron pressure, which, in turn, is roughly proportional to $p$ at constant $T$. The density, $\varrho$, is also proportional to $p$. Therefore, a local gas pressure reduction strongly affects the absorption coefficient, $\kappa \varrho$ (roughly $\kappa \varrho \propto p^{2}$ ). If a magnetic flux concentration with a radius $a \ll H_{p}$ is approximately in magnetohydrostatic equilibrium, the sum of the gas and magnetic pressures inside the structure equal the gas pressure at the same height outside (Spruit 1976). At low values of the plasma- $\beta$, the magnetic pressure makes up a significant fraction (namely $(1+\beta)^{-1}$ ) of the total pressure, entailing a strongly reduced gas pressure. Consequently, field concentrations make the gas locally more transparent and thus cause depressions in the optical surface, called "Wilson depressions" in analogy to the same phenomenon in sunspots (Bray and Loughhead 1964). This is a slightly oversimplified picture, as the local transparency changes entail changes in the local heat flux, which modify the temperature structure and have thus a considerable positive effect on the local value of $\kappa$, which counteracts the effect of $p$ on $\kappa \varrho$. Nonetheless, local depressions of the optical surface caused by magnetic flux concentrations have been invariably found in simulations of solar magneto-convection (e.g. Vögler et al. 2005, Cameron et al. 2007).

Figure 3.23 shows maps of $z\left(\tau_{\mathrm{R}}=1\right)$, i.e. maps of the geometrical depth of the optical surface, for four non-magnetic simulations and the respective $100 \mathrm{G}$ and $500 \mathrm{G}$ runs. The Wilson depressions show up very clearly as marked local minima in $z\left(\tau_{\mathrm{R}}=1\right)$ and coincide with the locations of strong flux concentrations (cf. Fig. 3.16).

Figure 3.24 illustrates the depth-dependence of the effect of the magnetic field on the 

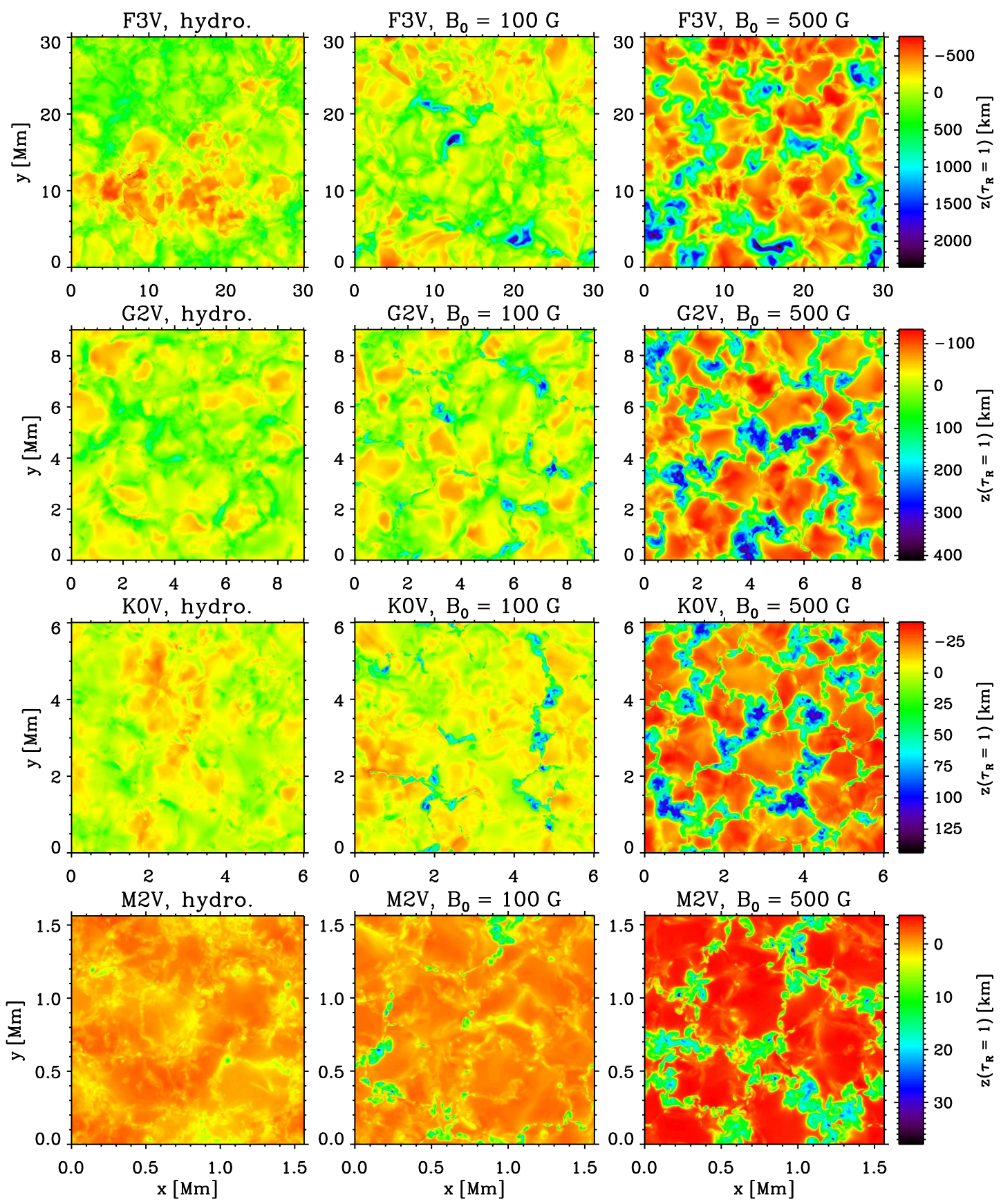

Figure 3.23: Maps of the geometrical depth of the optical surface for different spectral types and three different field strengths. Plots for K5V and M0V are given in Fig. B.5. 

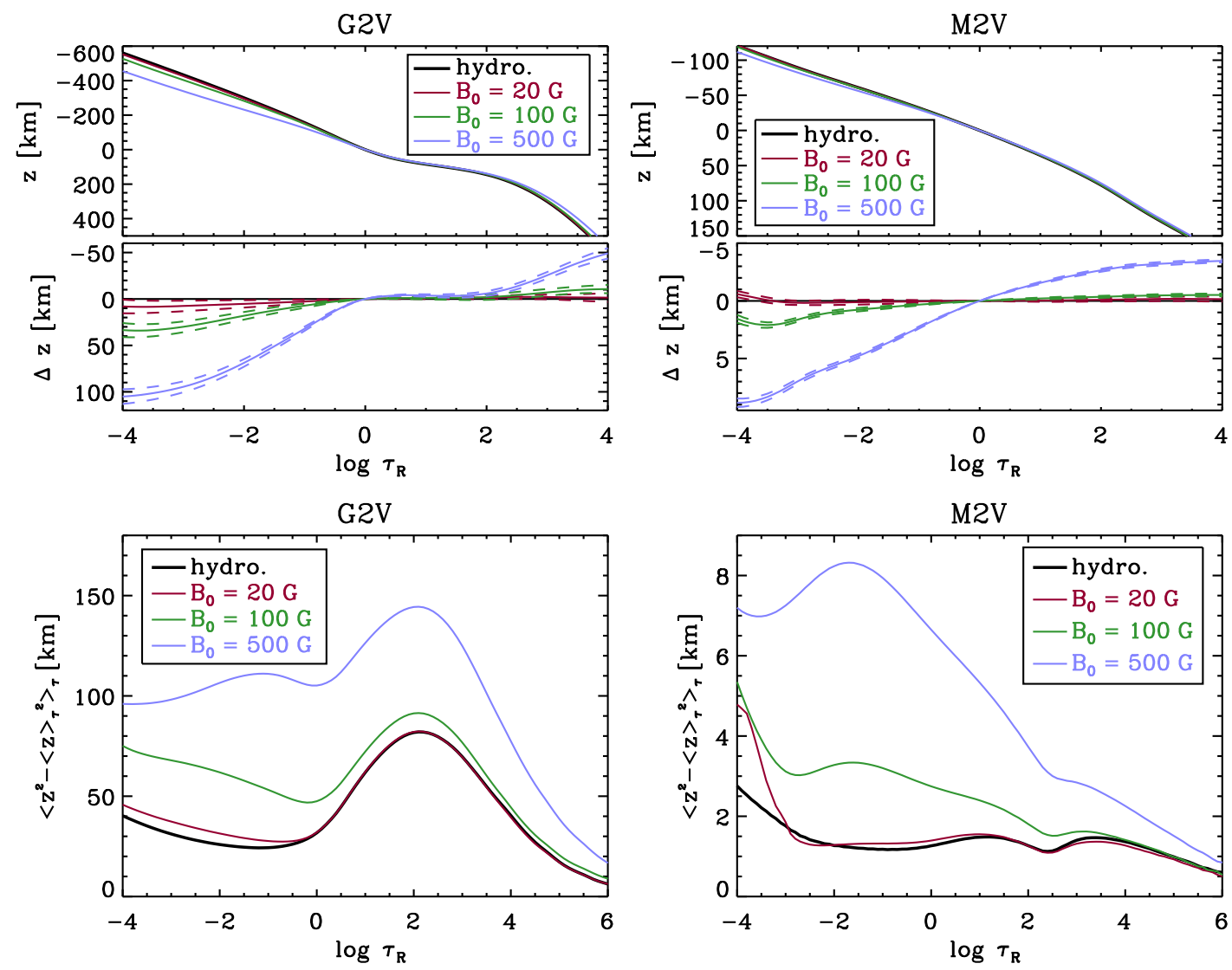

Figure 3.24: Top panels: average geometrical depth of iso- $\tau_{\mathrm{R}}$ surfaces in the G2V (left) and $\mathrm{M} 2 \mathrm{~V}$ (right) simulations. The black solid curve corresponds to the non-magnetic (hydro.) run; the lower subpanels show the differences between the magnetic runs and the non-magnetic run with $1 \sigma$ temporal scatter indicated by dashed lines. Bottom panels: rms fluctuations of the geometrical depth of iso- $\tau_{\mathrm{R}}$ surfaces in the G2V (left) and M2V (right) simulations. The black solid curve corresponds to the non-magnetic (hydro.) run.

iso- $\tau_{\mathrm{R}}$ surfaces for the $\mathrm{G} 2 \mathrm{~V}$ and $\mathrm{M} 2 \mathrm{~V}$ simulations. The upper panels show the average geometrical depth, $\left\langle z\left(\tau_{\mathrm{R}}\right)\right\rangle$, of these surfaces as function of $\log \tau_{\mathrm{R}}$. With increasing $B_{0}$, the iso- $\tau_{\mathrm{R}}$ surfaces move closer together, i. e. $\left|\left\langle z\left(\tau_{\mathrm{R}}\right)\right\rangle\right|$ is reduced somewhat by the presence of the magnetic field. For instance, in the $\mathrm{G} 2 \mathrm{~V}$ simulation with $B_{0}=500 \mathrm{G}$, the surface where $\log \tau_{\mathrm{R}}=-4$ is on average only $450 \mathrm{~km}$ above the optical surface compared to $550 \mathrm{~km}$ in the non-magnetic run, i. e. a relative difference of about $20 \%$. This can be regarded as an effect of the increasing dominance of the magnetic pressure with height (higher area fraction of regions with $\beta<1$ ): the magnetic flux concentrations fan out above the optical surface and the filling factor of the local depressions increases with height, reducing the average height of a given iso- $\tau_{\mathrm{R}}$ surface (see Fig. 3.27). As the photospheric gas pressure increases along the model sequence from $\mathrm{F} 3 \mathrm{~V}$ to $\mathrm{M} 2 \mathrm{~V}$, one expects this effect to decrease monotonically from F3V to M2V. In fact, the strongest effect is visible in the F3V star $\left(\sim 25 \%\right.$ reduction of $\left\langle z\left(\log \tau_{\mathrm{R}}=-4\right)\right\rangle$ at $\left.B_{0}=500 \mathrm{G}\right)$, however, it is weakest in the $\mathrm{K} 5 \mathrm{~V}$ simulation ( $\sim 4 \%$ reduction) and becomes somewhat stronger 

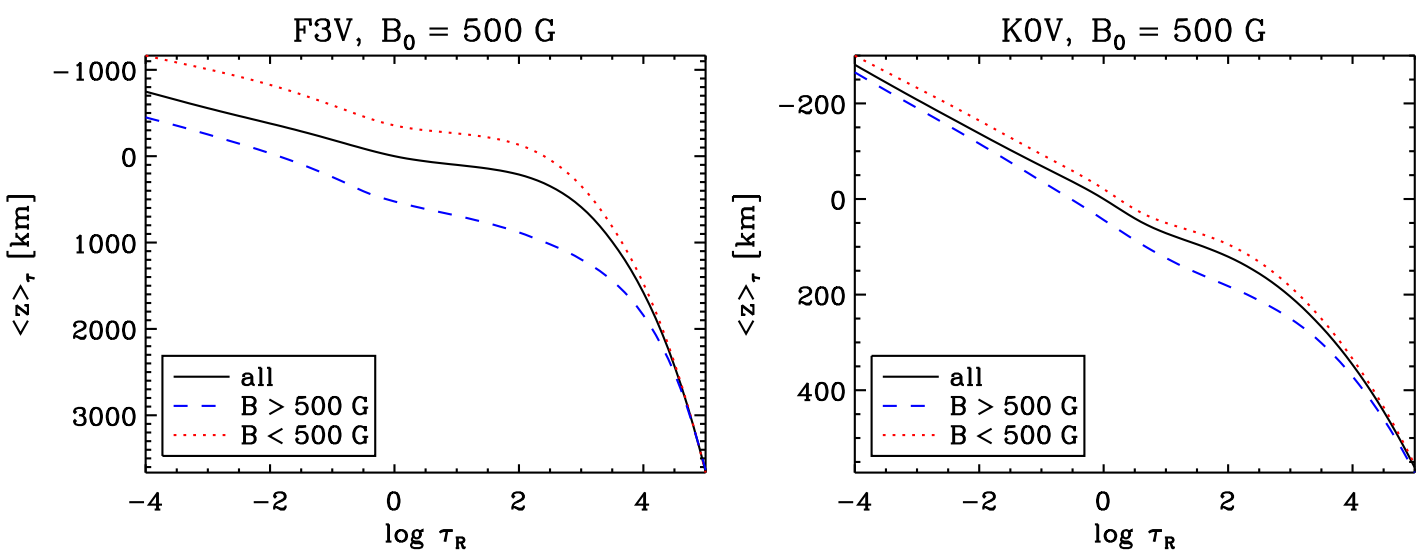

Figure 3.25: Average geometrical depth of iso- $\tau_{\mathrm{R}}$ surfaces in regions with $B>500 G$ (blue dashed curve), in regions with $B<500 \mathrm{G}$ (red dotted curve), and in the whole simulation box (black solid curve) for the $500 \mathrm{G}$ runs of the F3V (left) and K0V (right) simulations.

again in the M stars (for M2V: 8\% reduction). This is probably related to the effect of the magnetic field on the temperature (see Sect. 3.3.3.1): in the M-star simulations the horizontally averaged temperature in the photosphere is mostly lower in the $500 \mathrm{G}$ runs than in the non-magnetic runs, whereas, in the other stars, the horizontally averaged photospheric temperature increases with $B_{0}$. A higher temperature entails a higher pressure scale height, reducing the effect discussed above, while the lower temperature enhances the effect in the M stars.

The lower panels of Fig. 3.24 illustrate the effect of the magnetic field on the corrugation of the iso- $\tau_{\mathrm{R}}$ surfaces, i. e. the standard deviation, $\sigma\left(z\left(\tau_{\mathrm{R}}\right)\right):=\sqrt{\left\langle z\left(\tau_{\mathrm{R}}\right)^{2}\right\rangle-\left\langle z\left(\tau_{\mathrm{R}}\right)\right\rangle^{2}}$, of the geometrical depth of an iso- $\tau_{\mathrm{R}}$ surface. $\sigma\left(z\left(\tau_{\mathrm{R}}\right)\right)$ is higher by a factor of 2 to 5 (for $\log \tau_{\mathrm{R}} \lesssim 2$ ) in the $500 \mathrm{G}$ runs than in the non-magnetic runs for all spectral types. For the special case of $\tau_{\mathrm{R}}=1$, i. e. for the optical surface, this is already evident by the increasing amplitude of $z\left(\tau_{\mathrm{R}}=1\right)$ shown in Fig. 3.23. The strongest relative effect on the roughness of the optical surface is visible in the $\mathrm{M} 2 \mathrm{~V}$ star, where the optical surface is rather flat without magnetic field, namely $\sigma\left(z\left(\tau_{\mathrm{R}}=1\right)\right)=1.25 \mathrm{~km}$, and is substantially roughened by the magnetic field reaching $\sigma\left(z\left(\tau_{\mathrm{R}}=1\right)\right)=6.65 \mathrm{~km}$ in the $500 \mathrm{G}$ run. This is, however, still small compared to the local pressure scale height in that model (which is of the order of $25 \mathrm{~km}$ ). In the F3V star, where the optical surface is already quite strongly corrugated without magnetic field $\left(\sigma\left(z\left(\tau_{\mathrm{R}}=1\right)\right)=220 \mathrm{~km}\right)$, the roughness in the $500 \mathrm{G}$ run is $\sigma\left(z\left(\tau_{\mathrm{R}}=1\right)\right)=550 \mathrm{~km}$, exceeding the local pressure scale height (of the order of $350 \mathrm{~km})$.

The increased corrugation of the iso- $\tau_{\mathrm{R}}$ surfaces for higher values of $B_{0}$ is mainly a result of the Wilson depression of the magnetic flux concentrations. This is illustrated in Figure 3.25. The solid black curves of this figure indicate the depth dependence of $\left\langle z\left(\tau_{\mathrm{R}}\right)\right\rangle$ for the $500 \mathrm{G}$ runs of the F3V and K0V stars. The blue dashed curves and red dotted curves show the same quantity, but only averaged over regions where $B>500 \mathrm{G}$ 

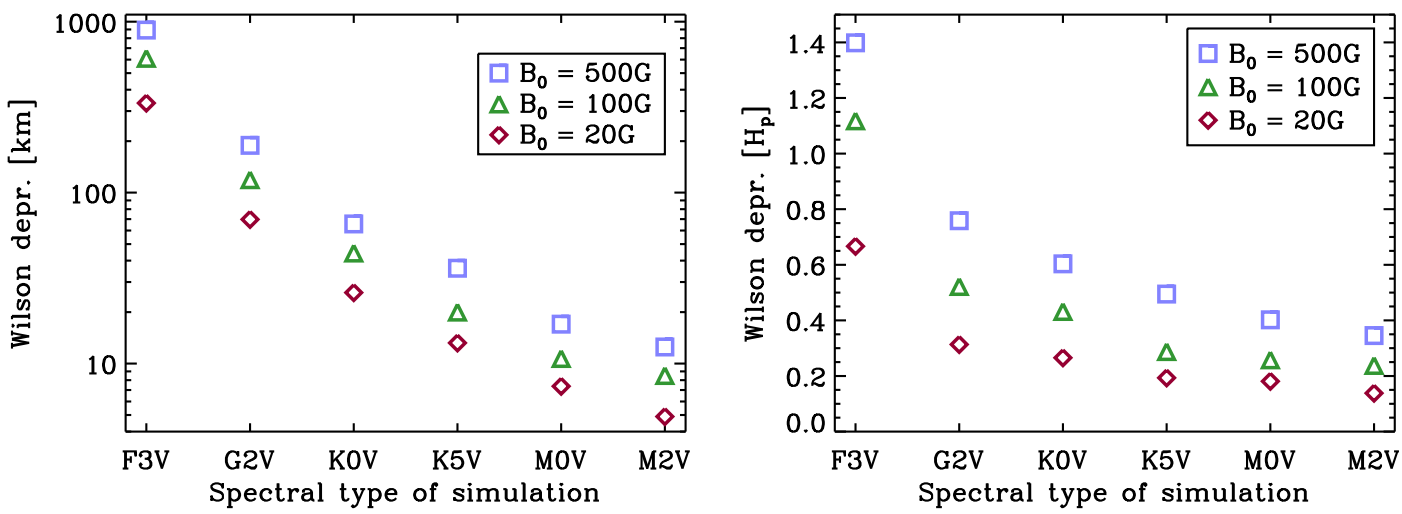

Figure 3.26: Wilson depressions. Left panel: Difference in average geometrical depth of the $\tau_{\mathrm{R}}=1$ surface between magnetic structures $\left(B\left(\tau_{\mathrm{R}}=1\right)>500 \mathrm{G}\right)$ and non-magnetic area $\left(B\left(\tau_{\mathrm{R}}=1\right)<500 \mathrm{G}\right)$ as measure for the depth of the Wilson depressions. Right panel: Downward shift of the optical surface within the magnetic flux concentrations in terms of pressure scale heights (measured outside the flux concentrations).

and $B<500 \mathrm{G}$, respectively. ${ }^{4}$ The blue dashed curves thus approximately represent the run of $\left\langle z\left(\tau_{R}\right)\right\rangle$ within the magnetic flux concentrations, while the red dotted curves show the same quantity in the less strongly magnetised parts of the simulation box. The difference between these curves gives an approximate measure of the typical depth of the local depressions of the iso- $\tau_{\mathrm{R}}$ surfaces due to magnetic field concentrations. For $\tau_{\mathrm{R}}=1$, this corresponds to the Wilson depressions (cf. Fig. 3.23). The left panel of Figure 3.26 shows this depth for all magnetic simulations. Along the model sequence, the depth of the Wilson depressions decreases by almost two orders of magnitude from $880 \mathrm{~km}$ in the F3V simulation to $12 \mathrm{~km}$ in the M2V simulation (both with $B_{0}=500 \mathrm{G}$ ). The right panel of Fig. 3.26 shows the depth of the Wilson depressions in terms of pressure scale heights outside the flux concentrations. As the pressure scale heights and the granule size differ only by roughly one order of magnitude between these two models, the depth of the Wilson depressions is also considerably larger in terms of pressure scale height or granule size in the F3V star compared to the M2V star. Figure 3.26 suggests that the average depth of the Wilson depressions depends on $B_{0}$. This is probably due to the fact that the average (horizontal) size of the magnetic flux concentrations becomes larger with larger $B_{0}$ and that the depth of a Wilson depression of a flux concentration depends somewhat on this size (see Fig. 3.23). This is partly caused by the fact that the remaining gas in the dark structures (which are larger than the bright structures) has a lower opacity owing to their lower temperature. Dark structures have consequently a more strongly depressed optical surface than bright structures for the same star. Partly, this effect might also be due to a selection bias: as all points on the optical surface with $B>500 \mathrm{G}$ where selected as belonging to a flux concentration, some selected points are located on the inclined side

\footnotetext{
${ }^{4}$ The value of $500 \mathrm{G}$ was chosen because it roughly corresponds to the minimum of the magnetic field distribution at the mean level of the optical surface (see Fig. 3.17), i.e. $500 \mathrm{G}$ approximately mark the transition from weak-field to strong-field regime.
} 
walls of the structures. Smaller structures have a higher fraction of these side-wall points.

Figure 3.27 shows vertical cuts through one magnetic flux concentration for each of the $100 \mathrm{G}$ simulations. The grey scale indicates density (normalised to the horizontally averaged density of the whole simulation box). The relative density perturbations become smaller from $\mathrm{F} 3 \mathrm{~V}$ to $\mathrm{M} 2 \mathrm{~V}$, as the average plasma- $\beta$ in the magnetic structures becomes larger. Consequently, the optical surface is less strongly depressed in the cooler models. The red dash-dotted contours show the surfaces of $T / T_{\text {eff }}=0.9,1.0$, and 1.1. In all flux concentrations, the $T=0.9 T_{\text {eff }}$ surface reaches higher up above the flux concentrations, indicating a heating of the upper part of the structure. Heating processes in these layers include adiabatic heating of the downflowing material, radiative heating from the inclined sidewalls of the depressed optical surface, viscous heating produced by vortex like motions or magneto-hydrodynamic waves, and ohmic heating in the current layer surrounding the flux concentration (cf. Figs. 3.34 and 3.35). The $T=1.1 T_{\text {eff }}$ surface, is approximately at the same height as the optical surface outside the flux concentrations. Within the flux concentrations it is above the optical surface in the three hottest simulations, but clearly below it in the M-star simulations. At this height, the radiative heating from the side walls of the depression is the most efficient heat source since the convective energy transport from below is supressed. The side-wall heating is more efficient, if the radius-to-depth ratio of the Wilson depression is small and if the sub-surface temperature gradient is large. The very shallow depressions of the magnetic flux concentrations in the M-star simulations consequently have a very limited side wall heating effect. The flux concentrations appear mainly as dark structures even at the lower values of $B_{0}$ in these simulations (see Figs. 3.15 and B.1). The various aspects of the heating in the magnetic flux concentrations is further discussed in Sects. 3.3.2 and 3.3.3.

\subsubsection{Velocity field}

The magnetic field has a strong effect on the velocity field. The Mach number in the simulations is mostly between 0.1 and 1 (see Fig. 3.5), which implies $B_{\text {eq, } p} \geq B_{\text {eq,kin }}$. Therefore, in regions with $\beta<1$ the field strength is generally above equipartition with the thermal and kinetic energy densities. $\beta$ decreases with increasing height in the simulated stars (see Fig. 3.20). Therefore, it is expected that the flows in the high atmospheric layers are more strongly affected by the magnetic forces than the flows in the deeper, optically thick parts of the simulation boxes. At and below the optical surface, the very inhomogeneous distribution of the magnetic field (cf. Figs. 3.16 and 3.22) suggests that downflows are more strongly affected by the field than upflows in the deeper layers.

Figure 3.28 shows the rms of the vertical and horizontal flow velocity components for the $\mathrm{G} 2 \mathrm{~V}$ and $\mathrm{M} 2 \mathrm{~V}$ simulations on horizontal planes as functions of normalised pressure as depth coordinate. The results for the other stars are qualitatively similar. The rms of the vertical flow speed, $v_{z, \text { rms }}$, is reduced in the magnetic runs below the optical surface. For the $500 \mathrm{G}$ runs, this reduction reaches up to $\sim 20 \%$ in F3V and G2V and $\sim 25 \%$ in the Kand M- star simulations. In contrast, about three to five pressure scale heights above the optical surface, $v_{z, \text { rms }}$ is increased in most magnetic runs compared to the the non-magnetic runs. Here, even at $B_{0}=20 \mathrm{G}$, the rms of the vertical speed is increased by $10-20 \%$ in the $\mathrm{G} 2 \mathrm{~V}$ and M2V simulations. For the runs with $B_{0}=100 \mathrm{G}$, the strongest increase of $v_{z \text {,rms }}$ in the upper photosphere is visible in most simulations: $v_{z, \text { rms }}$ is increased by $\sim 60 \%$ in 

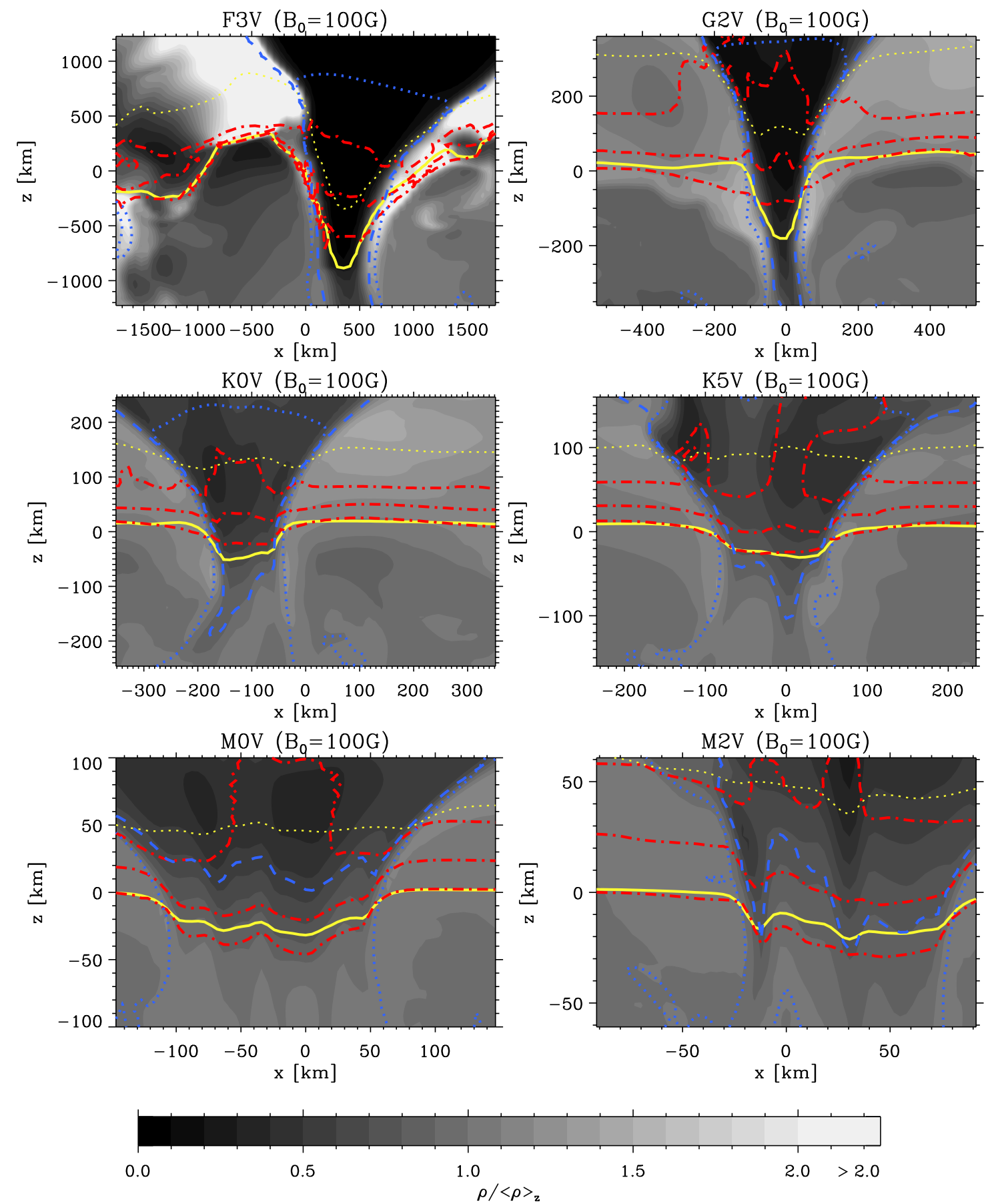

Figure 3.27: Cuts through typical magnetic flux concentrations in the six simulations with $B_{0}=100 \mathrm{G}$. The grey scale indicates the density $\varrho /\langle\varrho\rangle_{z}$ relative to the horizontal mean density; the solid (dotted) yellow curve is the $\tau_{\mathrm{R}}=1\left(\tau_{\mathrm{R}}=0.01\right)$ surface; the dashed blue contour shows the $\beta=1$ surface; the dotted blue curve is the $500 \mathrm{G}$ iso- $B$ surface; the red dash-dotted curves indicate the $T / T_{\text {eff }}=0.9,1.0$, and 1.1 surfaces. $x$ and $z$ coordinates are to scale for each panel but differ more than one order of magnitude for the different models. 

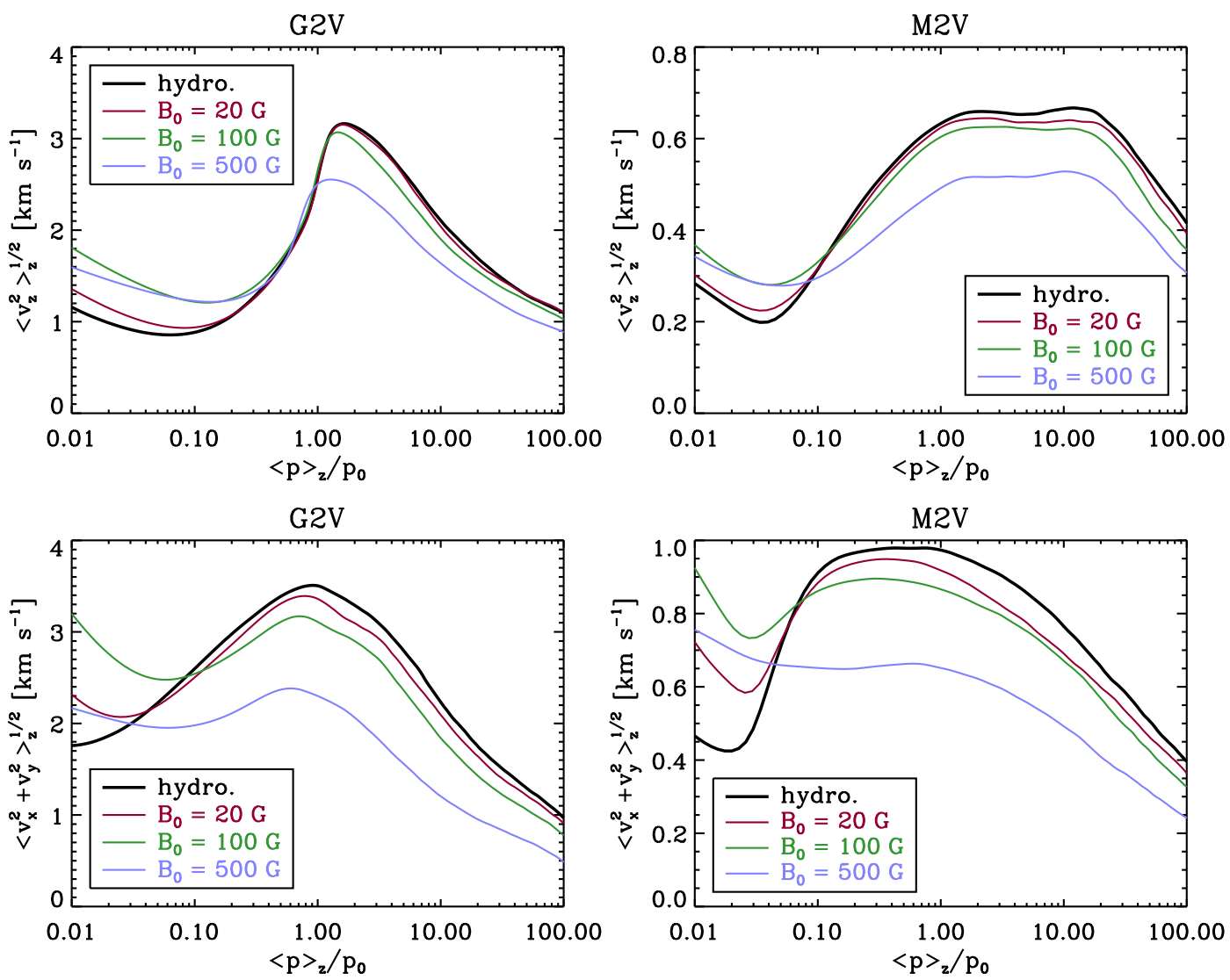

Figure 3.28: Run of the rms of the vertical (upper panels) and horizontal (lower panels) components of the flow velocity for the G2V (left panels) and $\mathrm{M} 2 \mathrm{~V}$ (right panels) simulations.

the G2V simulation (25\% in the K0V simulation, $\sim 15 \%$ in the K5V simulation, and $30-$ $40 \%$ in the M-star simulations). Only in the F3V simulation the rms of the vertical flow speed is up to $20 \%$ smaller for all three magnetic simulations in the upper photosphere $\left(p \lesssim 0.1 p_{0}\right)$.

In general, $v_{\text {hor,rms }}:=\sqrt{v_{x}^{2}+v_{y}^{2}}$ shows a similar depth dependence as $v_{z, \text { rms }}$ (see lower panels of Fig. 3.28). Its reduction below the surface in the magnetic runs is somewhat stronger (for $B_{0}=500 \mathrm{G}$ : $\sim 35 \%$ in the $\mathrm{F} 3 \mathrm{~V}$ and the $\mathrm{G} 2 \mathrm{~V}$ simulation, $\sim 40 \%$ in the $\mathrm{K}$ and M-star simulations) and is already noticable at $B_{0}=20 \mathrm{G}$. The increase about three to five pressure scale heights above the optical surface is also stronger. Again, the $100 \mathrm{G}$ runs show the strongest effect in these layers and the rms of the horizontal velocities is increased by 60 to $100 \%$ in all simulations with the exception of $\mathrm{F} 3 \mathrm{~V}$ and $\mathrm{K} 5 \mathrm{~V}$, where this increase is relatively small $(\sim 10 \%)$.

In the optically thick lower layers of the simulation boxes, the main effect of the magnetic field is a reduction of the velocity in the downflows, while the upflows are much less affected as expected from the preference of the magnetic field for the downflows. This is illustrated in Figure 3.29, where the depth-dependence of the average upflow and downflow speed is shown for the G2V and M2V simulations. The upflow speed below 

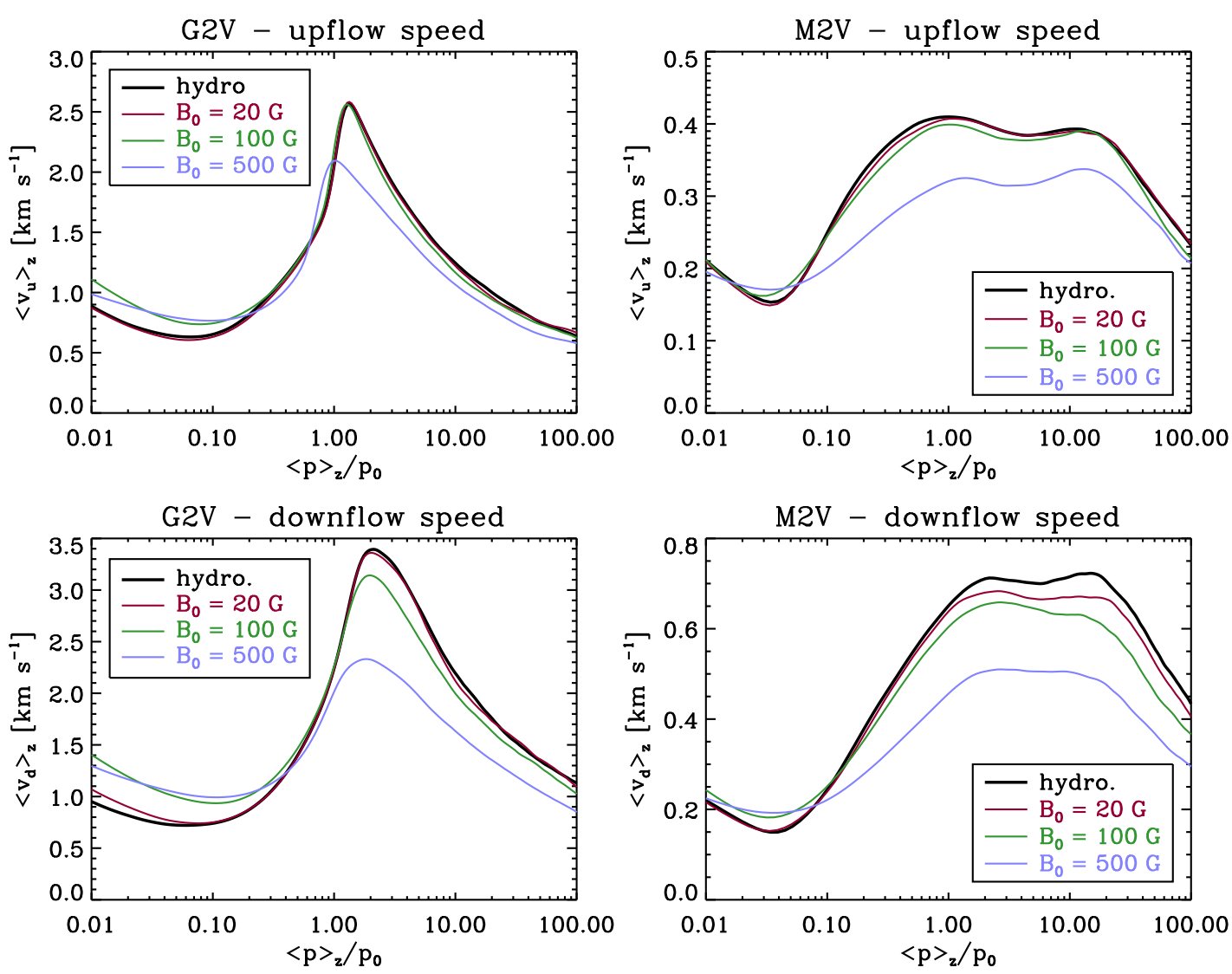

Figure 3.29: Horizontal averages of the upflow (upper panels) and downflow (lower panels) speeds in the G2V (left panels) and M2V (right panels) simulations.

the optical surface is reduced by $10-20 \%$ at $B_{0}=500 \mathrm{G}$ in these two stars. For the simulatons of other spectral types, the effect is similar. The downflow speed is reduced somewhat more strongly: all simulations show a reduction of about $30 \%$ of the downflow speed in the first few pressure scale heights below the optical surface at $B_{0}=500 \mathrm{G}$ compared to the respective non-magnetic run. Figure B.21 in Appendix B shows the upand downflow speeds averaged on surfaces of constant optical depth.

As the downflow speed is more strongly reduced by the presence of the magnetic field than the upflow speed and the density in the downflows is also reduced, it is expected that the area fraction of upflows, which is about $65-70 \%$ in the non-magnetic runs (cf. Fig. 3.6), should be lower in the $500 \mathrm{G}$ runs. This is actually the case: in all simulations with $B_{0}=500 \mathrm{G}$, the area fraction of upflows is reduced to values around $60 \%$ below the optical surface.

Figure 3.30 shows maps of the vertical component $v_{z}(z=0)$ of the flow velocity at the mean level of the optical surface for the non-magnetic and two magnetic $\left(B_{0}=\right.$ $100 \mathrm{G}, 500 \mathrm{G}$ ) runs for the F3V, G2V, K0V, and M2V simulations (maps for the K5V and M0V simulations are given in Fig. B.6 in Appendix B). For the magnetic simulation runs, the time step shown is the same as in Figs. 3.15, 3.16, 3.21, and 3.22. On the one hand, the presence of the magnetic field appears to reduce the amount of small-scale structure 

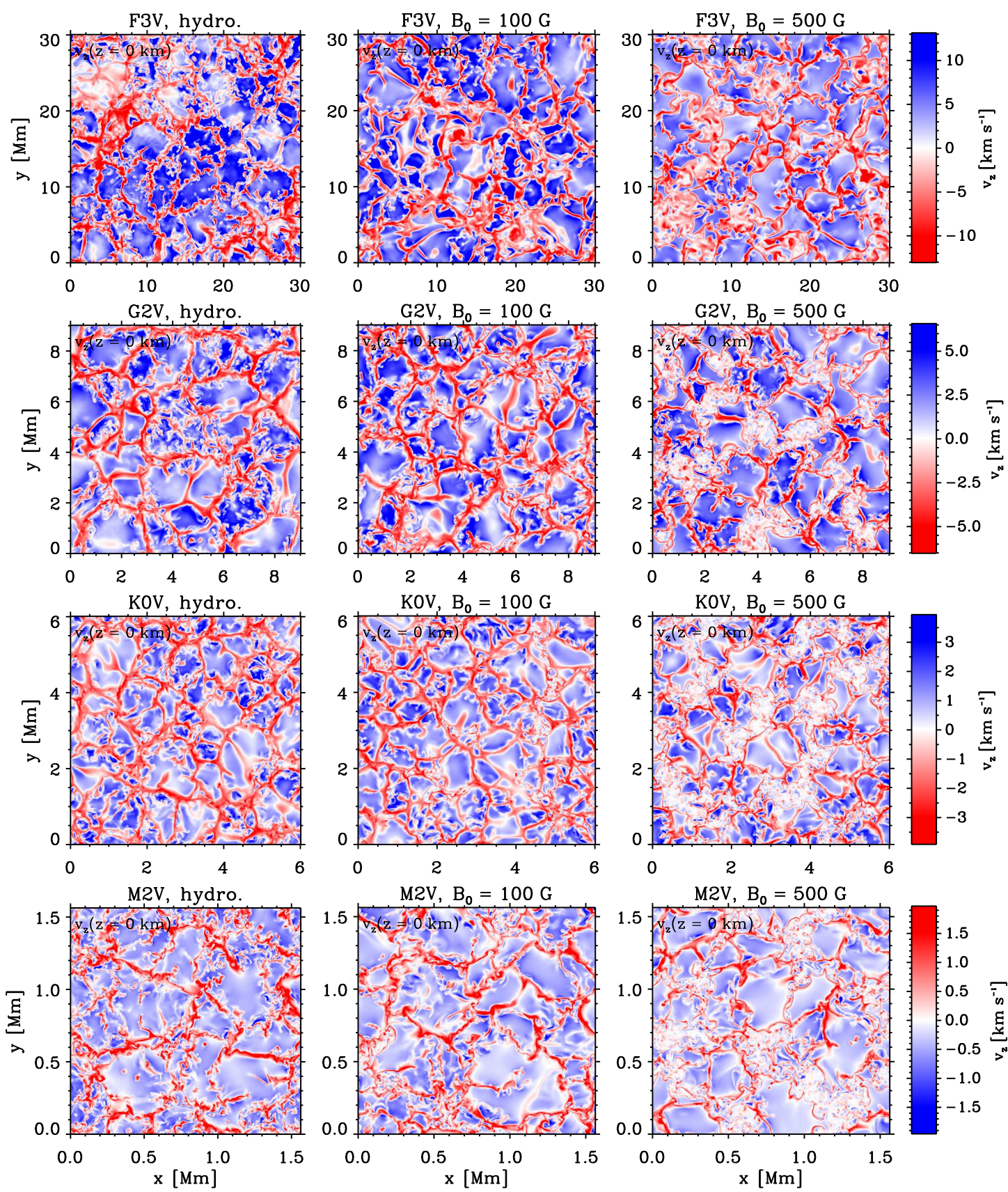

Figure 3.30: Vertical component, $v_{z}$, of the flow velocity for four different spectral types (from top to bottom: $\mathrm{F} 3 \mathrm{~V}, \mathrm{G} 2 \mathrm{~V}, \mathrm{~K} 0 \mathrm{~V}$, and $\mathrm{M} 2 \mathrm{~V}$; for $\mathrm{K} 5 \mathrm{~V}$ and M0V, see Fig. B.6) for three different runs per spectral type (from left to right: non-magnetic (hydro.), $B_{0}=100 \mathrm{G}$, and $\left.B_{0}=500 \mathrm{G}\right)$ at the average level of the optical surface $(z=0)$.

(especially well visible in the $100 \mathrm{G}$ run of the F3V star, which looks much smoother than its non-magnetic counterpart). On the other hand, strong concentrations of magnetic field appear as regions with detailed small-scale structure in $v_{z}$, albeit with reduced $v_{z}$ amplitude. Small amounts of magnetic flux apparently help to stabilise downflows as the field is mainly vertical and thus Lorentz force mainly acts on horizontal flows. Larger 
flux concentrations start to impede even the vertical flows, as the mass supply at the top of the structures is blocked. After an initial formation phase during which the downflow evacuates the upper part of the structure, the velocity at the optical surface drops almost to zero. Moreover, if $\beta<1$, the complicated structure of the flows and magnetic field in deeper layers is partly imposed on the velocity structure at the surface.

Figure 3.31 shows the joint histogram of the vertical components of the magnetic field and the flow velocity for the same selection of simulations as in Fig. 3.19, taken from a layer of half a pressure scale height each above and below the mean level of the optical surface. The vertical component of the magnetic field has a similar bimodal distribution as its modulus $|B|$ (cf. Fig. 3.17) in all simulations. The correlation between $B_{z}$ and $v_{z}$ is qualitative different between the spectral types: in the F3V-star simulation, the vertical component of the flow velocity has a broad distribution in the weak- and in the strongfield parts of the simulation. Most of the upflows are associated with weak field. In the strong-field regime around $B_{z} \approx 2 \mathrm{kG}$, there occur downflows of up to $12 \mathrm{~km} \mathrm{~s}^{-1}$, but also a small percentage of substantial upflows. In the cooler $\mathrm{K} 0 \mathrm{~V}$-star simulation, there is a very clear relation between $B_{z}$ and $v_{z}$ : for $\left|B_{z}\right|<0.5 \mathrm{kG}$, there is a broad distribution of $v_{z}$, while for $0.5 \mathrm{kG}<B_{z}<2 \mathrm{kG}$ the vertical flow speed is mostly negative, its modulus decreasing with increasing $B_{z}$. For $B_{z}>2 \mathrm{kG}, v_{z}$ is mostly close to zero. For the coolest model of the sequence, the M2V-star simulation, the picture is similar, but the distribution of $v_{z}$ around 0 for high field strength is even narrower and extends down to approximately $B_{z}=1 \mathrm{kG}$.

These different joint histograms can be understood in terms of lifetimes of magnetic flux concentrations. As the magnetic field is concentrated in downflows, one would, on the one hand, expect the strongest fields to coincide with the strongest downdrafts. On the other hand, these downflows cannot be sustained owing to the suppression of their horizontal inflows, i. e. of their mass supply. Consequently, a downflow is only possible during the formation phase and not in a long-lived flux concentration. The downflows are thus decelerated by the presence of the field. Long-lived magnetic regions are almost at rest. As is shown in Sect. 4.3.3, the magnetic flux concentrations on the M-star simulations evolve on much longer timescales than the flux concentrations on hotter stars, which is consistent with the different appearance of the histograms in Fig. 3.31.

Figures 3.32 and 3.33 show vertical velocity maps for four depths below the optical surface for the non-magnetic and the 100 and $500 \mathrm{G}$ runs of the G2V and M2V simulations (for the other simulations see Figs. B.7 - B.10 in Appendix B). While in the non-magnetic simulations the network of intergranular lanes breaks apart into a number of strong downdrafts below the surface, the downflows seem to prefer a more networklike structure in the magnetic case down to 4.6 pressure scale heights below the optical surface. This phenomenon is known for solar magnetoconvection (Nordlund and Stein 1990). The strongest magnetic flux concentrations (cf. Fig. 3.16) all appear above extended downflow areas. The "mesogranular" structure indicated by the locations of magnetic flux concentrations is thus a reflection of the structure of the subsurface flow pattern. As already shown in Figs. 3.28 and 3.29, the average speeds decrease with increasing $B_{0}$ in these layers. As the magnetic field prevents most downdrafts to become as fast as in the non-magnetic case, the area fraction of downflows has to increase and a more networklike structure evolves. This might also be one of the causes for the slightly smoother appearance of the vertical flow structure at the optical surface (cf. Fig. 3.30). 

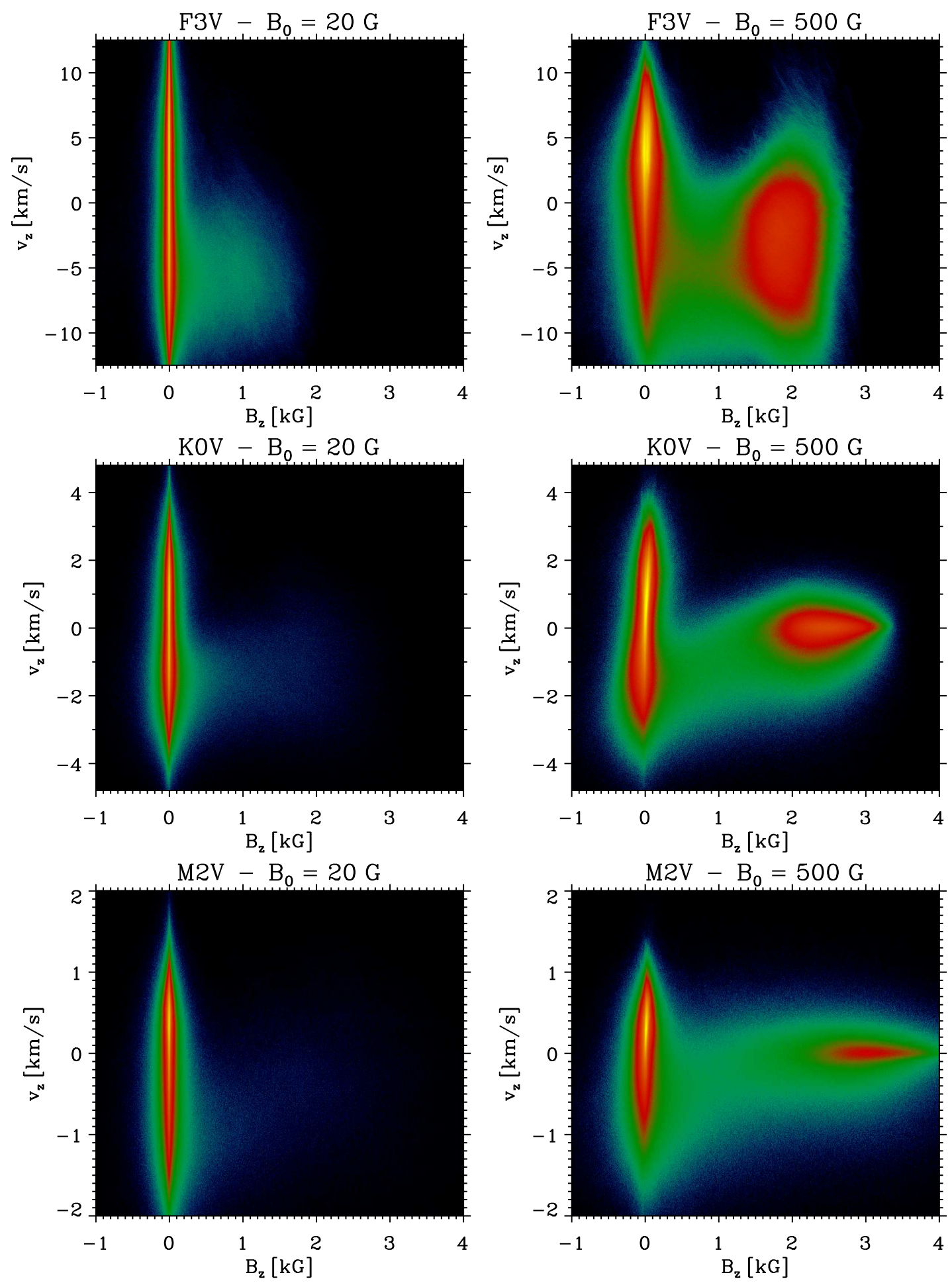

Figure 3.31: Joint histograms of the vertical components of the magnetic field and the flow velocity in a layer between $\left\langle z\left(p=p_{0} \exp (-0.5)\right)\right\rangle$ and $\left\langle z\left(p=p_{0} \exp (+0.5)\right)\right\rangle$, where $p_{0}=\left\langle p\left(\tau_{\mathrm{R}}=1\right)\right\rangle$ is the mean pressure at the optical surface. 

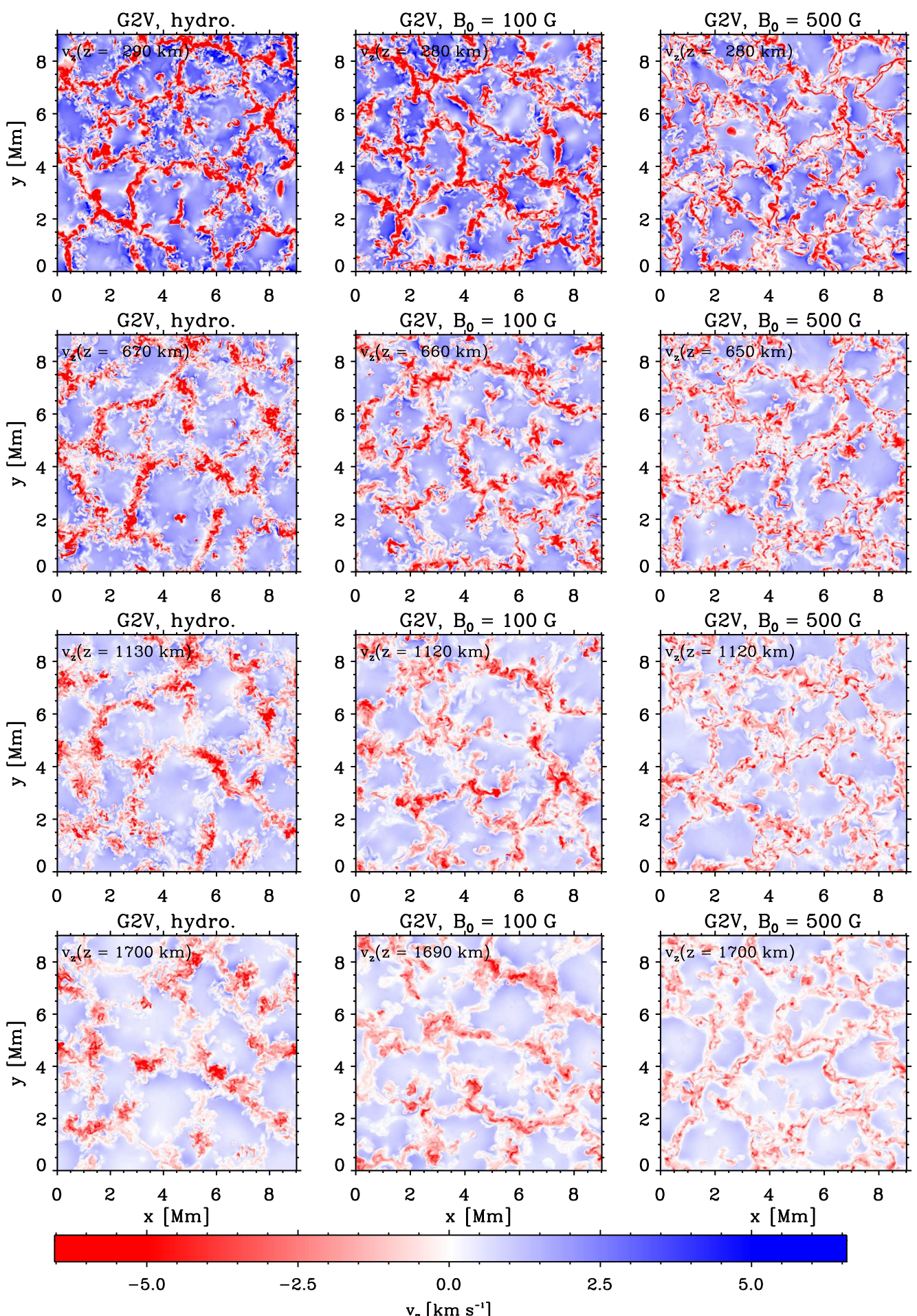

Figure 3.32: Vertical component, $v_{z}$, of the flow velocity at four distinct depth levels below the optical surface for the G2V simulation without magnetic field (left column), with $B_{0}=100 \mathrm{G}$ (middle column), and with $B_{0}=500 \mathrm{G}$ (right column). The four depth levels correspond to roughly $1.15,2.3,3.45$, and 4.6 pressure scale heights below the optical surface (from top to bottom); the geometrical depth is given in each panel; also see Figs. 3.33 and B.7 - B.10. 

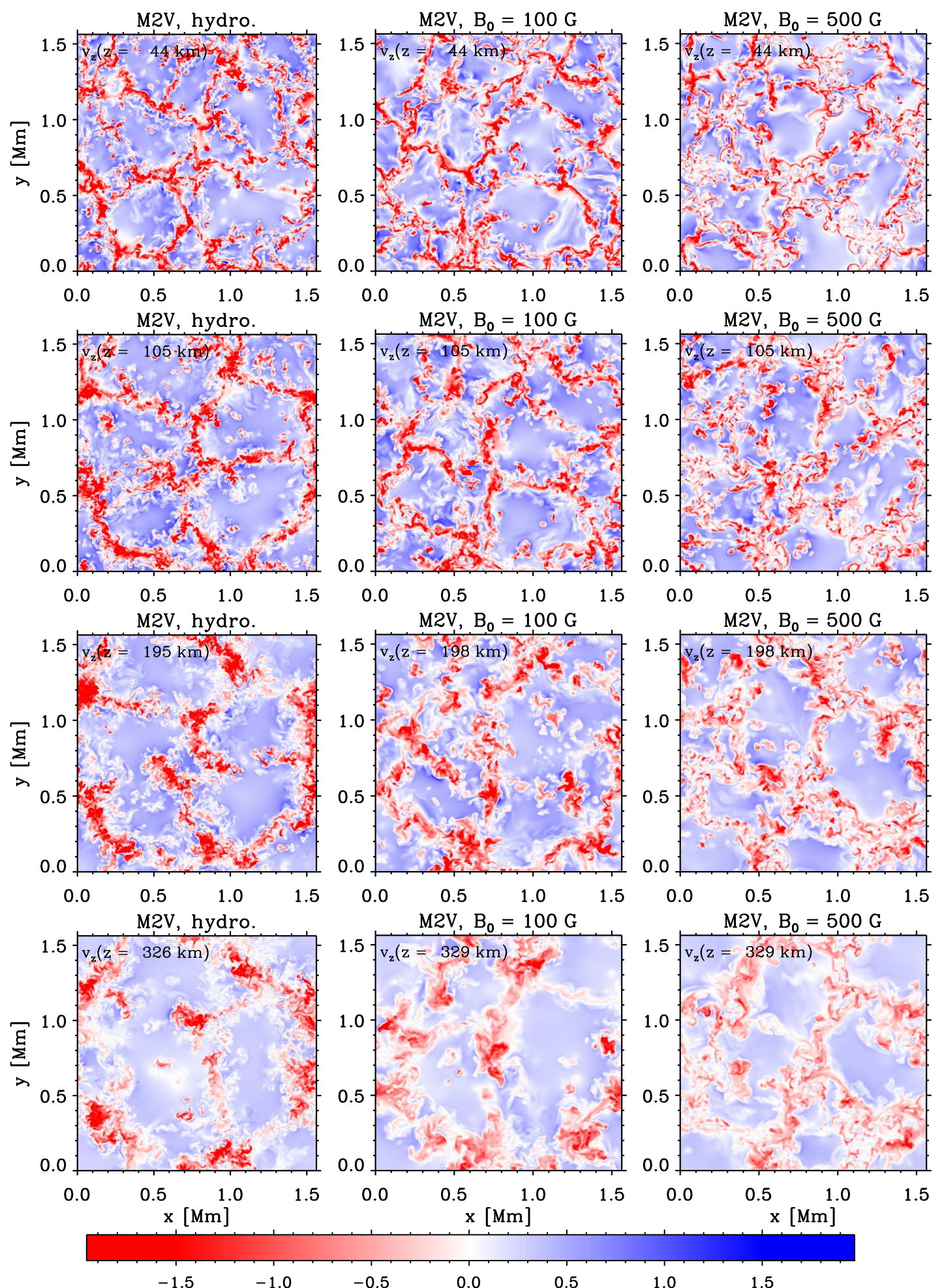

Figure 3.33: Same as Fig. 3.32, but for the M2V simulation. Plots for the F3V, K0V, K5V, and MOV simulations are given in Figs. B.7 - B.10. 

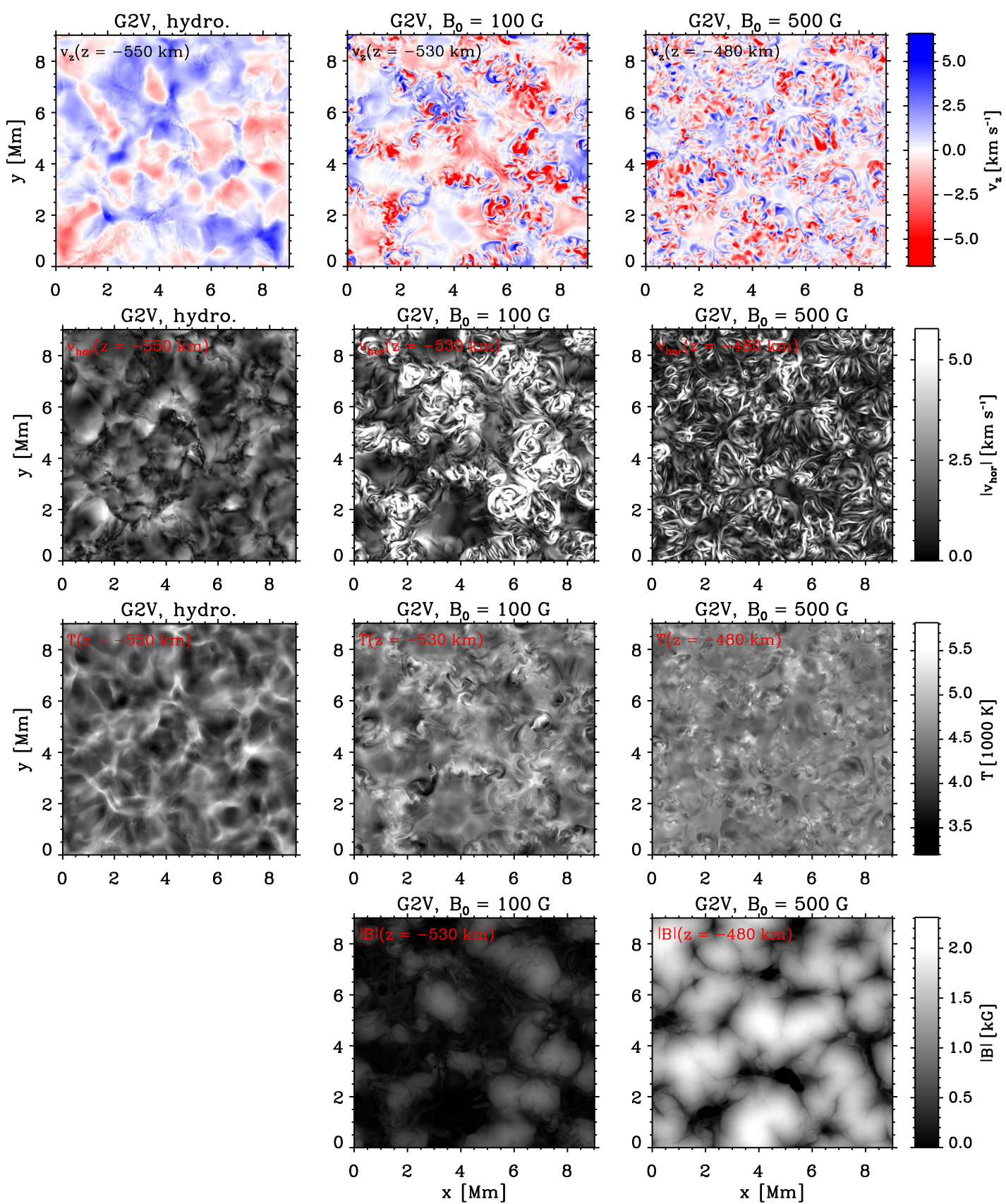

Figure 3.34: Horizontal cuts through three different runs of the G2V star simulation without magnetic field (left column) with $B_{0}=100 \mathrm{G}$ (middle column) and with $B_{0}=500 \mathrm{G}$. All cuts are situated such that $\langle p\rangle_{z}=0.01 p_{0}$, i. e. about 4.6 pressure scale heights above the optical surface; the geometrical depth of this level is given in each panel. From top to bottom: Vertical velocity, $v_{z}$, horizontal speed, $\left|v_{\text {hor }}\right|:=\left(v_{x}^{2}+v_{y}^{2}\right)^{1 / 2}$, Temperature, $T$, and magnetic field strength $|B|$ are given; also see Figs. 3.35 and B.11 - B.14. 

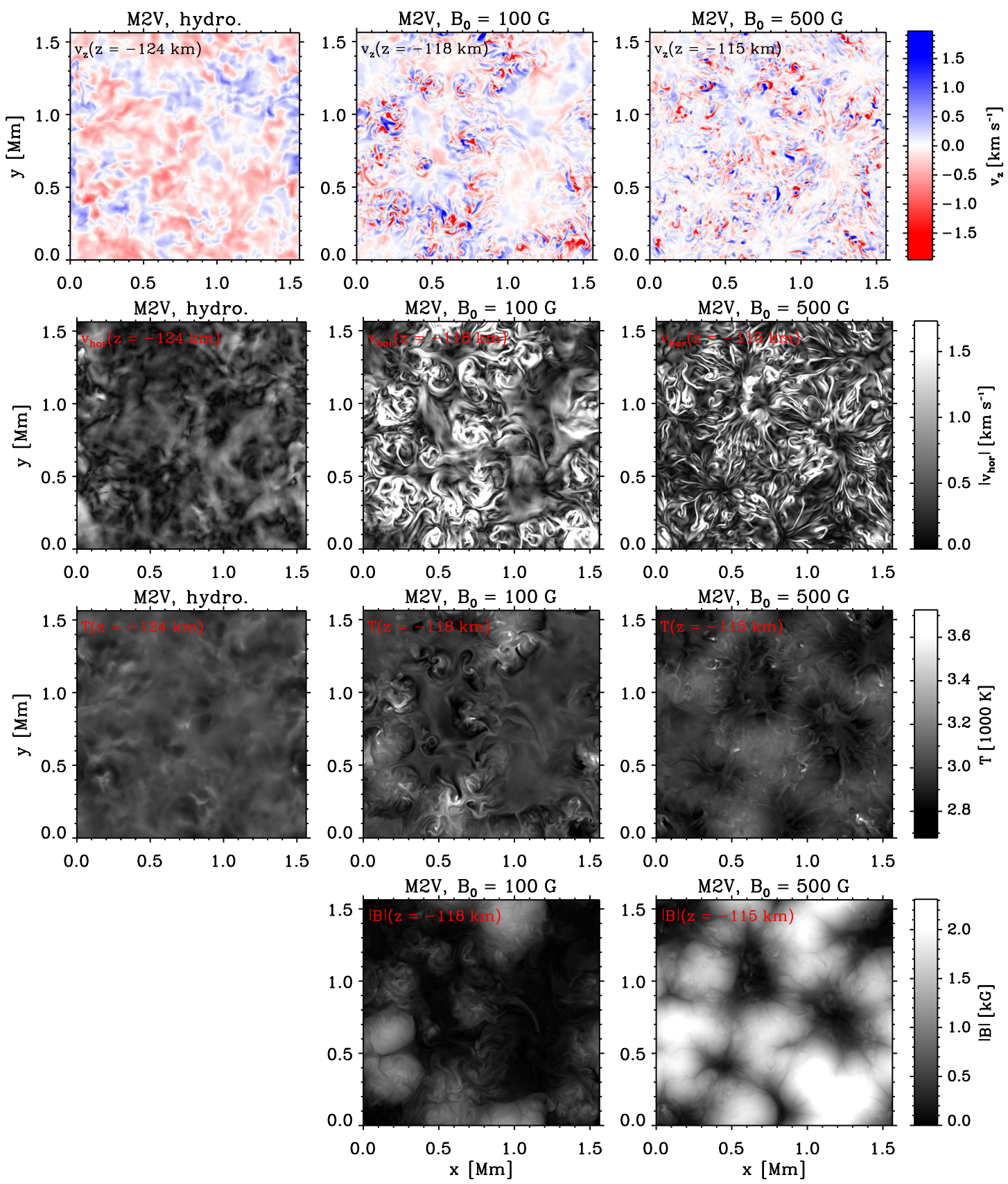

Figure 3.35: Same as Fig. 3.34, but for the M2V star; plots for the F3V, K0V, K5V, and MOV simulations are given in Figs. B.11 - B.14. 
Figures 3.34 and 3.35 illustrate the dynamics of the higher atmosphere, which change considerably from the non-magnetic to the magnetic case. For the same simulation runs as in Figs. 3.32 and 3.33, the figures show the vertical component, $v_{z}$, of the flow velocity, the modulus of the horizontal flow speed, $\left|v_{\text {hor }}\right|=\sqrt{v_{x}^{2}+v_{y}^{2}}$, the temperature, $T$, and the modulus of the magnetic field strength, $|B|$, in horizontal cuts situated 4.6 pressure scale heights above the optical surface (for the same figure for the other four stellar types, see Figs. B.11 - B.14 in Appendix B). In this high layer, the $\beta<1$ regions comprise a high area fraction and the magnetic field governs the dynamics of the plasma. Without magnetic field, the vertical velocity structure in this height is dominated by overshooting convection, which penetrates these layers from the strongest upflows. As the sound speed decreases with increasing height (decreasing temperature), some of the flows become supersonic and shocks evolve at the interface between up- and downflows in the $\mathrm{G} 2 \mathrm{~V}$ simulation. The viscous heating at the shock fronts is the main non-radiative heat source in this layer (also see Moll et al. 2011). If a magnetic field is present, the velocity structure of the upper atmosphere becomes much more complicated, as the magnetic field couples these layers to the lower layers: above magnetised downflows of the $100 \mathrm{G}$ run, strong downflows with vortex-like horizontal motions evolve. The sharp and straight shock fronts dissappear, but in some of the vortices there is substantial viscous heating (also see Moll et al. 2012). Moreover, the plasma motions in the deeper layers may excite various magnetohydrodynamic wave modes (Jafarzadeh et al. 2013, Shelyag et al. 2013). Consequently, the sites of this non-radiative heating (i. e. of enhanced $T$ ) roughly coincide with the locations of magnetic field concentrations. At $500 \mathrm{G}$, the velocity amplitudes and heating goes down, as strong magnetic field (especially the magnetic tension force) starts to suppress the formation of vortices and strong downflows near the optical surface. In the M2V star, the situation is rather similar. Here, the magnetic field seems to be even more important for the heating of the upper atmosphere, as there are no shocks in the non-magnetic case. The sites of enhanced temperature in the cut shown in Fig. 3.35 coincide very well with the sites where the magnetic field strength is particularly high for both magnetic runs shown.

\subsubsection{Thermodynamic structure}

In Sect. 3.3.1 it was shown that the magnetic field has a strong effect on the local density and temperature near the optical surface (cf. Fig. 3.27). At the end of Sect. 3.3.2, the influence of the magnetic field on the thermodynamical structure in the upper atmosphere was briefly described (cf. Figs. 3.34 and 3.35). In this section, the effect of the magnetic field on $T(x, y, z), p(x, y, z)$, and $\varrho(x, y, z)$ is discussed in more detail.

\subsubsection{Temperature}

The effective temperature of the simulations slightly changes due to the presence of the magnetic field (see Table 2.1 and Fig. 2.2). As described in Sect. 2.3.3, the bottom boundary condition for the entropy density in the simulations was left unchanged when a magnetic field was introduced. This is close to the real situation, if the boxes are deep enough and if the magnetised regions are thought to be relatively small and thus not to substantially influence the global structure of the star. However, one should be cautious 

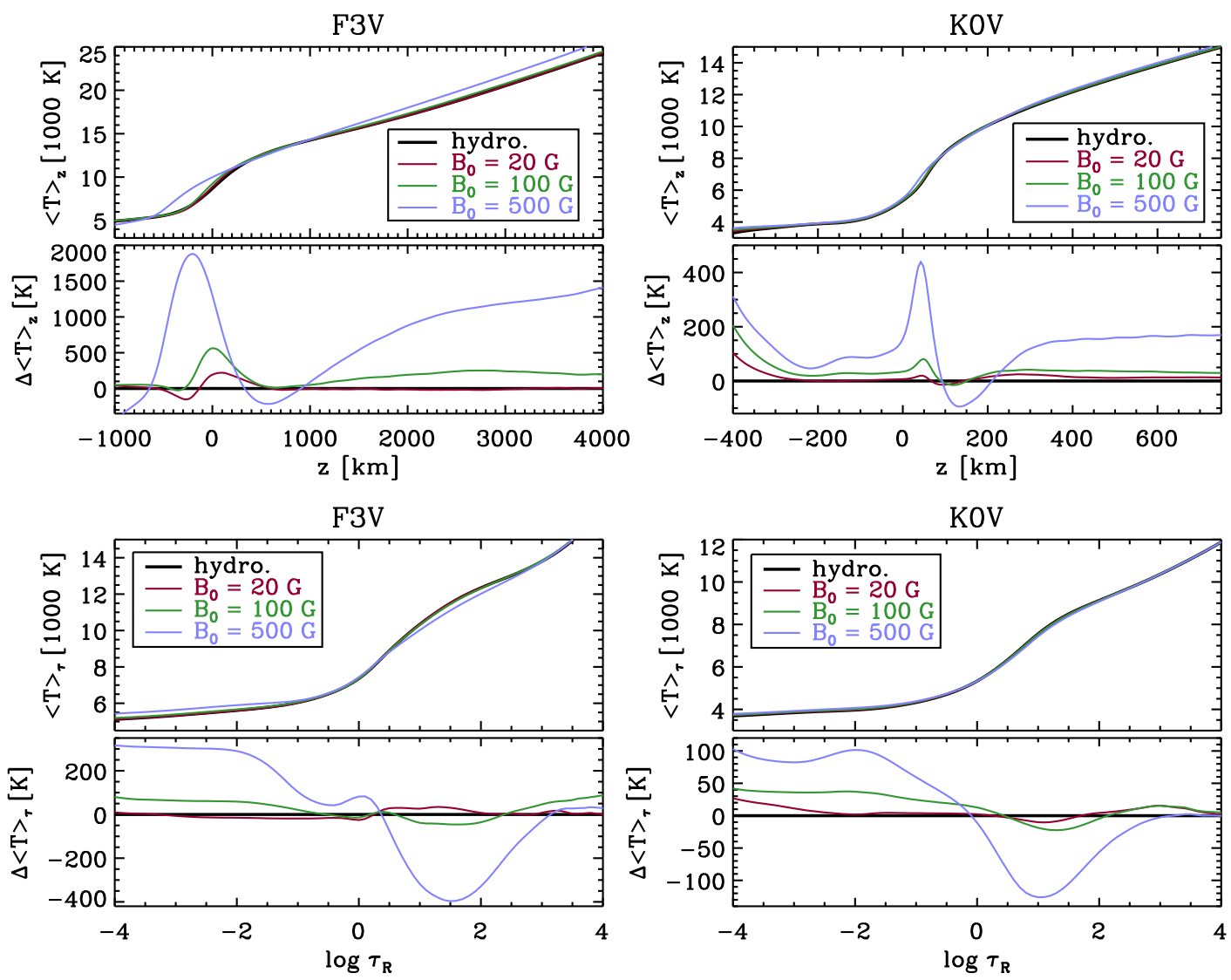

Figure 3.36: Run of the horizontally averaged temperature as function of $z$ (upper panels) and $\log \tau_{\mathrm{R}}$ (lower panels) for the $\mathrm{F} 3 \mathrm{~V}$ (left) and $\mathrm{KOV}$ (right) simulations. The lower subplot displays the differences between the magnetic runs and the non-magnetic (hydro.) run.

to interpret these differences in effective temperature to reflect the absolute radiative flux differences to be expected between active regions and the quiet unmagnetised star. The differences in effective temperature of the runs of different $B_{0}$ should be taken into account in the interpretation of the synthetic spectral line profiles.

Figure 3.36 illustrates the depth dependence of the temperature in the F3V and K0V simulations averaged over horizontal planes (upper panels) and over surfaces of constant optical depth (lower panels). In horizontal planes, the temperature is higher in the magnetic runs than in the non-magnetic runs at almost all depths. In the F3V star, the temperature in the $500 \mathrm{G}$ run is higher than in the non-magnetic run by more than $1000 \mathrm{~K}$ at depths $z \geq 3000 \mathrm{~km}$. In the lower atmosphere, there seems to be an increase of almost $2000 \mathrm{~K}$ from non-magnetic to $500 \mathrm{G}$ run in this star. However, one has to take into account the very corrugated optical surface: the deep Wilson depressions of the magnetic flux concentrations shift the average level of the optical surface (which defines $z=0$ ) outside the magnetic flux concentrations downwards. This mimics a strong increase of $T(z)$ as the steep photospheric temperature gradient of the weakly magnetised regions is moved to a shallower geometrical depth. The run of the temperature averaged on iso- $\tau_{\mathrm{R}}$ surfaces, i.e. $\langle T\rangle_{\tau}\left(\tau_{\mathrm{R}}\right)$, indicates a somewhat weaker, but still considerable, impact of 

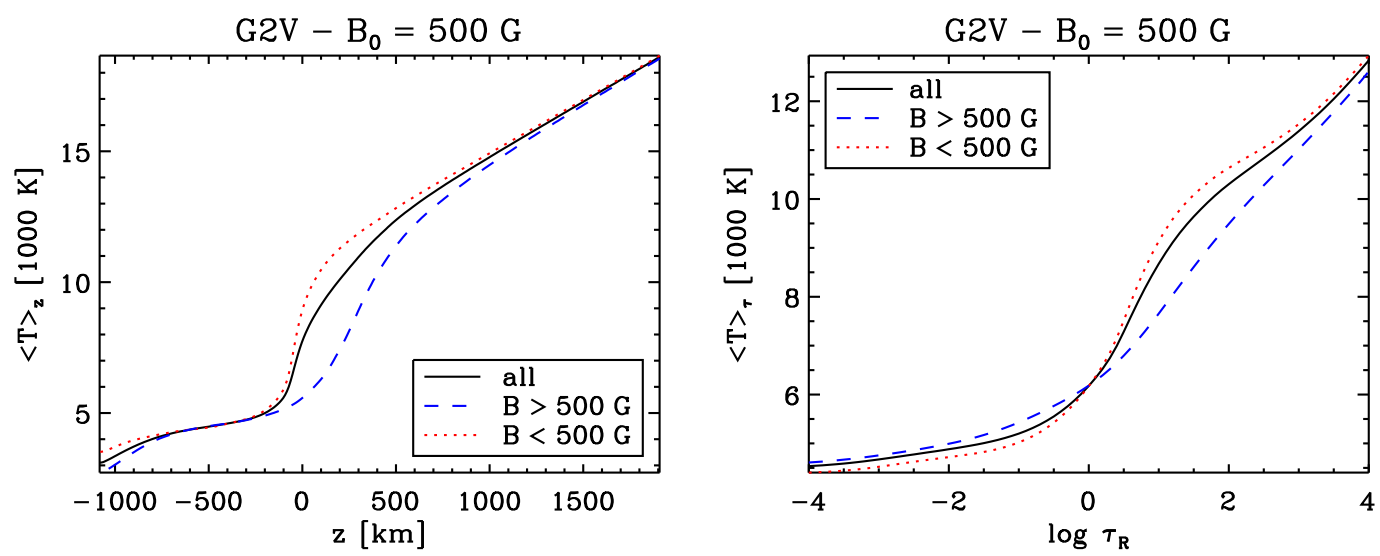

Figure 3.37: Temperature averaged on horizontal planes (left panel) and surfaces of constant optical depth (right panel) where $B>500 \mathrm{G}$ (blue dashed curve) and where $B<500 \mathrm{G}$ (red dotted curves) for the $500 \mathrm{G}$ run of the G2V simulation. The black solid curve gives the averages over the total area.

the magnetic field on the depth dependence of temperature. The difference in $\langle T\rangle_{\tau}\left(\tau_{\mathrm{R}}\right)$ between simulations with different $B_{0}$ becomes insignificant only in the deepest layers $\left(\log \tau_{\mathrm{R}} \gtrsim 3\right)$ for all spectral types. Slightly below the optical surface the magnetic runs are cooler (especially the $500 \mathrm{G}$ run), and in the higher atmospheric layers they are hotter than the non-magnetic runs. In the M-stars, the $500 \mathrm{G}$ runs are cooler (in iso- $\tau_{\mathrm{R}}$ averages between $\log \tau_{\mathrm{R}}=3$ and $\log \tau_{\mathrm{R}}=-2$.

Figure 3.37 shows the run of the temperature in regions with $B>500 \mathrm{G}$ and with $B<$ $500 \mathrm{G}$ for the $\mathrm{G} 2 \mathrm{~V}$ simulation with $B_{0}=500 \mathrm{G}$. The horizontally averaged temperature $\langle T\rangle_{z}$ is much lower within magnetic flux concentrations than in regions which are only weakly magnetised. This is again an effect of the depressions caused by the magnetic flux concentrations (cf. Figs. 3.25 and 3.27). The temperature averaged over iso- $\tau_{\mathrm{R}}$ surfaces (see right panel of Fig. 3.37) is higher within the flux concentrations in the atmosphere $\left(\tau_{\mathrm{R}} \lesssim 1\right)$, but lower in the convective envelope $\left(\tau_{\mathrm{R}} \gg 1\right)$. This is partly due to the fact that most of the magnetic flux is accumulated in downflows: downflows are hotter than upflows in the subadiabatically stratified atmospheres while they are cooler than upflows in the (superadiabatically stratified) convective envelopes. In order to isolate the effect of the magnetic field, one has to compare downflows and upflows separately between the $500 \mathrm{G}$ run and the non-magnetic runs. This comparison is shown in Fig. 3.38 for the $\mathrm{F} 3 \mathrm{~V}, \mathrm{G} 2 \mathrm{~V}, \mathrm{~K} 0 \mathrm{~V}$, and $\mathrm{M} 2 \mathrm{~V}$ simulations. Interestingly, the effect of the magnetic field on the temperature is qualitatively and quantitatively quite similar in up- and downflows and thus they both resemble approximately the overall effect shown in Fig. 3.37: the atmospheres are hotter in the magnetic simulations while the layers directly below the surface are cooler (if compared at the same optical depth). Generally, the effect on the downflows is somewhat stronger than the effect on the upflows. Only the M stars show a considerably different impact: in the magnetic simulations only a very small thin layer in the atmospheres (around $\log \tau_{\mathrm{R}}=-3$ ) is hotter than in the non-magnetic simulations while the lower (and very high) atmosphere is cooler in up- and downflows.

As pointed out in Sect. 3.2, not all downflows carry substantial magnetic flux. Fig- 

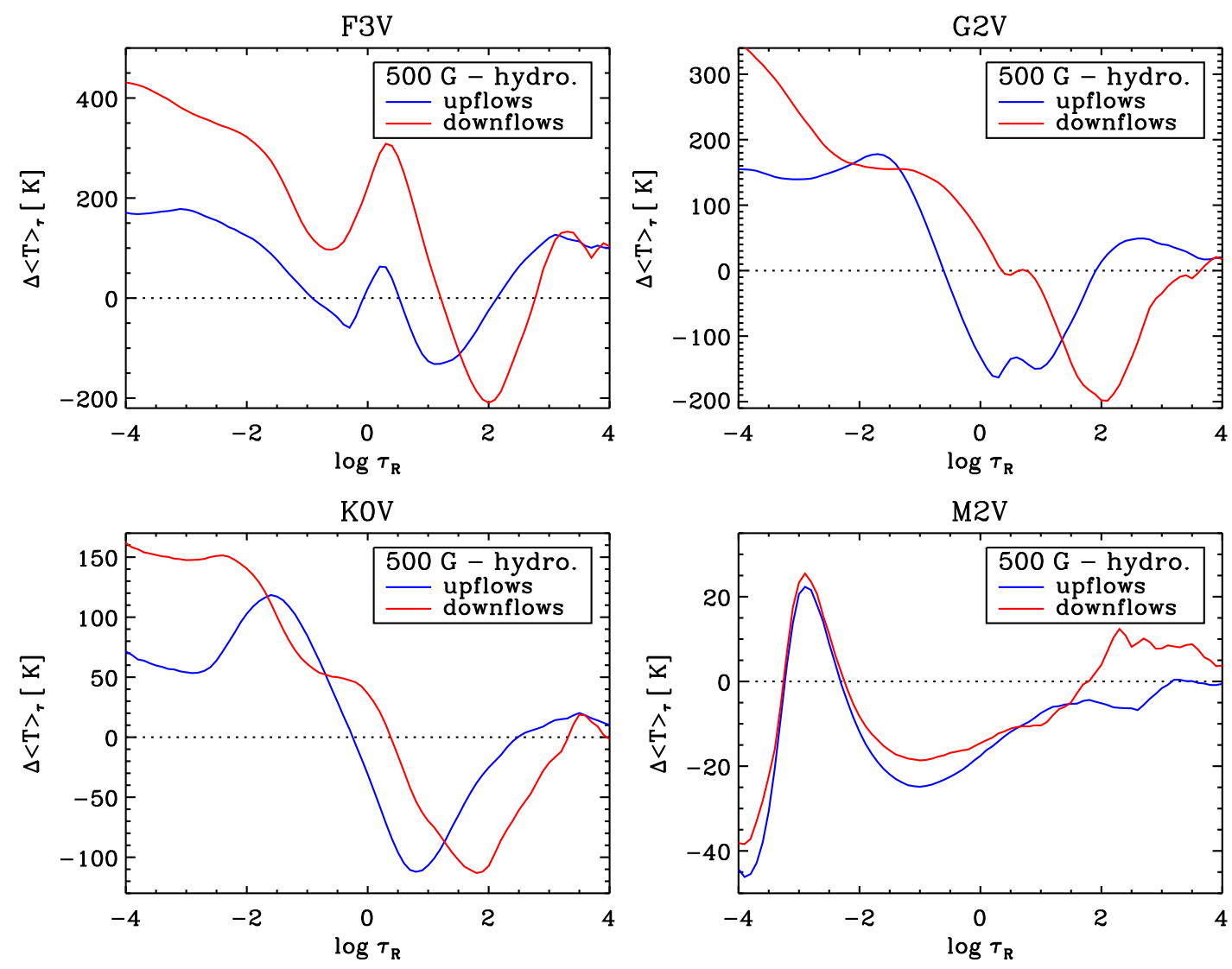

Figure 3.38: Average temperature difference between the $500 \mathrm{G}$ and non-magnetic runs in up- and downflows for the $\mathrm{F} 3 \mathrm{~V}, \mathrm{G} 2 \mathrm{~V}, \mathrm{~K} 0 \mathrm{~V}$, and $\mathrm{M} 2 \mathrm{~V}$ simulations.

ure 3.39 shows $\langle T\rangle_{\tau}\left(\tau_{\mathrm{R}}\right)$ separately for weakly magnetised downflows $(B<500 \mathrm{G})$ and for strongly magnetised downflows $(B>500 \mathrm{G})$ in the $500 \mathrm{G}$ run of the K0V star relative to the non-magnetic simulation of that star. The strongly magnetised downflows are further decomposed into dark structures $\left(I_{\mathrm{bol}}<\left\langle I_{\mathrm{bol}}\right\rangle-\sigma_{I}\right.$, where $\sigma_{I}$ denotes the standard deviation of $\left.I_{\mathrm{bol}}\right)$, bright structures $\left.\left(I_{\mathrm{bol}}\right\rangle\left\langle I_{\mathrm{bol}}\right\rangle+\sigma_{I}\right)$, and intermediately bright structures $\left(\left\langle I_{\mathrm{bol}}\right\rangle-\sigma_{I} \leq I_{\mathrm{bol}} \leq\left\langle I_{\mathrm{bol}}\right\rangle+\sigma_{I}\right)$. As expected, $\langle T\rangle_{\tau}\left(\tau_{\mathrm{R}}\right)$ differs strongly between differently bright structures. However, all three groups of strongly magnetised downflows have a lower temperature than the weakly magnetised downflows around $\log \tau_{\mathrm{R}}=2$ and a higher temperature above $\log \tau_{\mathrm{R}}=-2$. The reduced temperature in the magnetic structures below the surface is probably caused by the reduced mixing between up- and downflows due to the suppression of horizontal motions, while the enhanced temperature above the surface is caused by various heat sources, especially the radiative heating from the side walls of the depression.

Figure 3.40 illustrates the temperature structure of the $100 \mathrm{G}$ run of the K0V star and the $500 \mathrm{G}$ run of the M2V star as two examples. The figure shows maps of $T\left(x, y, \tau_{\mathrm{R}}\right)$ for a number of different optical depths for a fraction of the horizontal extent of the two simulation boxes. All temperature images are saturated at \pm 2 standard deviations from the mean temperature of the respective map. The values for the mean and the satura- 

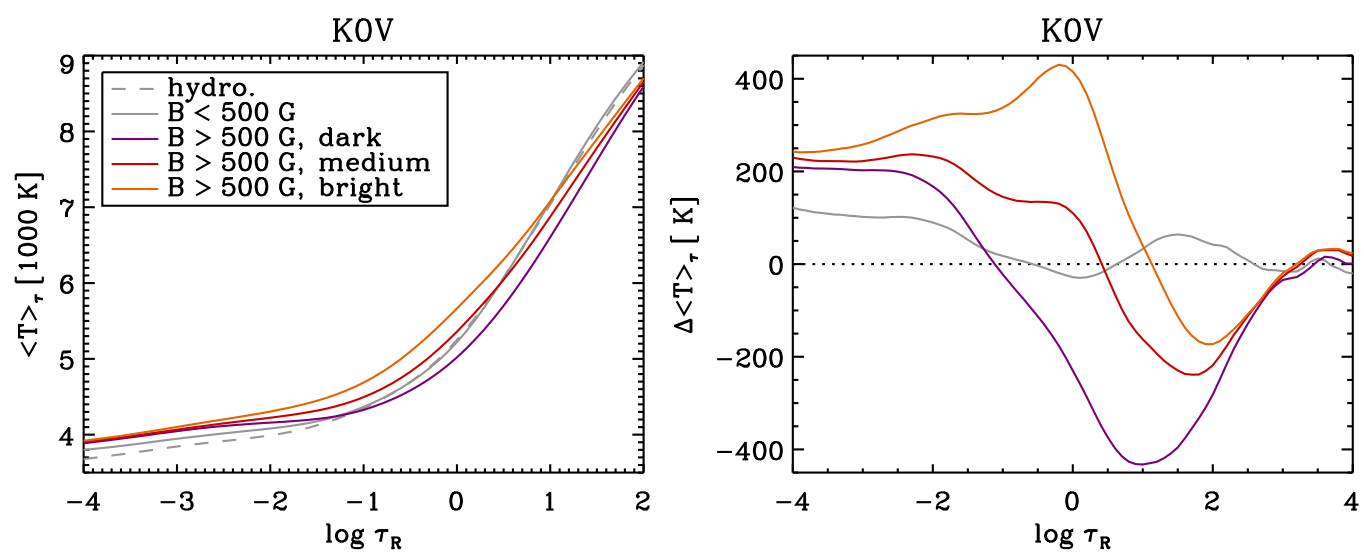

Figure 3.39: Left panel: Temperature averaged on surfaces of constant optical depth in the downflows of the $500 \mathrm{G}$ and non-magnetic runs for the K0V simulations. The downflows of the magnetic run are divided into different components: a weakly magnetised component $(B<500 \mathrm{G}$; grey curve), and three strongly magnetised components $(B>500 \mathrm{G})$ with different vertical bolometric intensity: low intensity ( dark, $\hat{I}<-1$, purple curve), medium intensity (medium, $-1 \leq \hat{I} \leq 1$, red curve), and high intensity (bright, $\hat{I}>1$, orange curve), using the definition of $\hat{I}$ given in Eq. (4.1). Right panel: Difference between the curves of the left panel and the averaged temperature of the non-magnetic run (grey dashed curve in left panel).

tion thresholds are indicated on the color bar next to each map. The snapshots are the same as shown in Figs. 3.15 and 3.16. The region shown for the K0V simulation contains several very bright magnetic features. These features are remarkably hot directly at the optical surface. Not much below the surface, at $\log \tau_{\mathrm{R}}=1.5$, these structures are, however, much cooler than the surrounding granules and even somewhat cooler than weakly magnetised downflows owing to the reduced mixing below up- and downflows. This is consistent with the average temperature profiles of magnetised downflows shown in Fig. 3.39. Above the optical surface, at $\log \tau_{R}=-1.5$ the temperature fluctuations of the non-magnetised area is reversed, as the (overshooting) upflows above granules are cooler than the downflows. The atmosphere above most of the bright magnetic structures is hotter than average from the optical surface up to $\log \tau_{\mathrm{R}}=-4.5$, again consistent with Fig. 3.39. In the higher layers, there appear some vortex-like structures (e.g. at $(x, y)=(3.2 \mathrm{Mm}, 4.9 \mathrm{Mm})$; cf. Fig. B.12). The region shown for the M2V simulation contains parts of the most prominent dark magnetic structure as well as a second, smaller and less dark one and the surrounding granules. At the optical surface, the very dark region is cooler by up to $200 \mathrm{~K}$ than the average temperature, while the smaller dark region is mostly only cooler by $50-100 \mathrm{~K}$. Both structures are surrounded by very small-scaled hot features. Below the surface, the dark structures are still cooler than the environment but not significantly cooler than less magnetised downflows. The dark structures remain also cooler somewhat above the optical surface (cf. Fig. 3.39), but at $\log \tau_{\mathrm{R}} \lesssim-3$, they appear as hot regions (especially the smaller one). At $\log \tau_{\mathrm{R}}=-3$ and $\log \tau_{\mathrm{R}}=-4.5$, the coolest regions are above the granules (reversed granulation).

In the F- G- and K-star simulations, the magnetic flux concentrations are mostly 
$\log \tau_{\mathrm{R}}=-4.5$
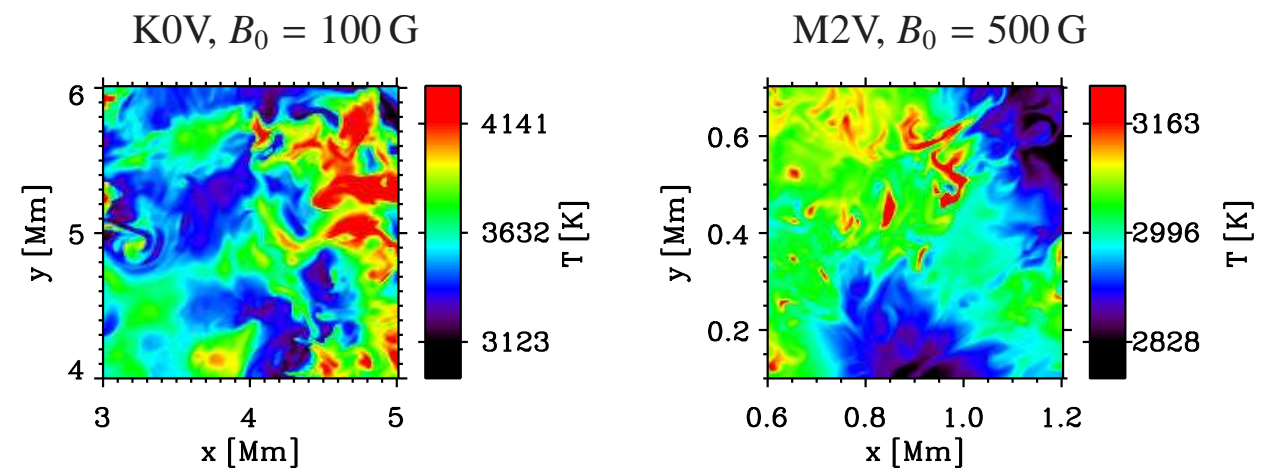

$\log \tau_{\mathrm{R}}=-3.0$
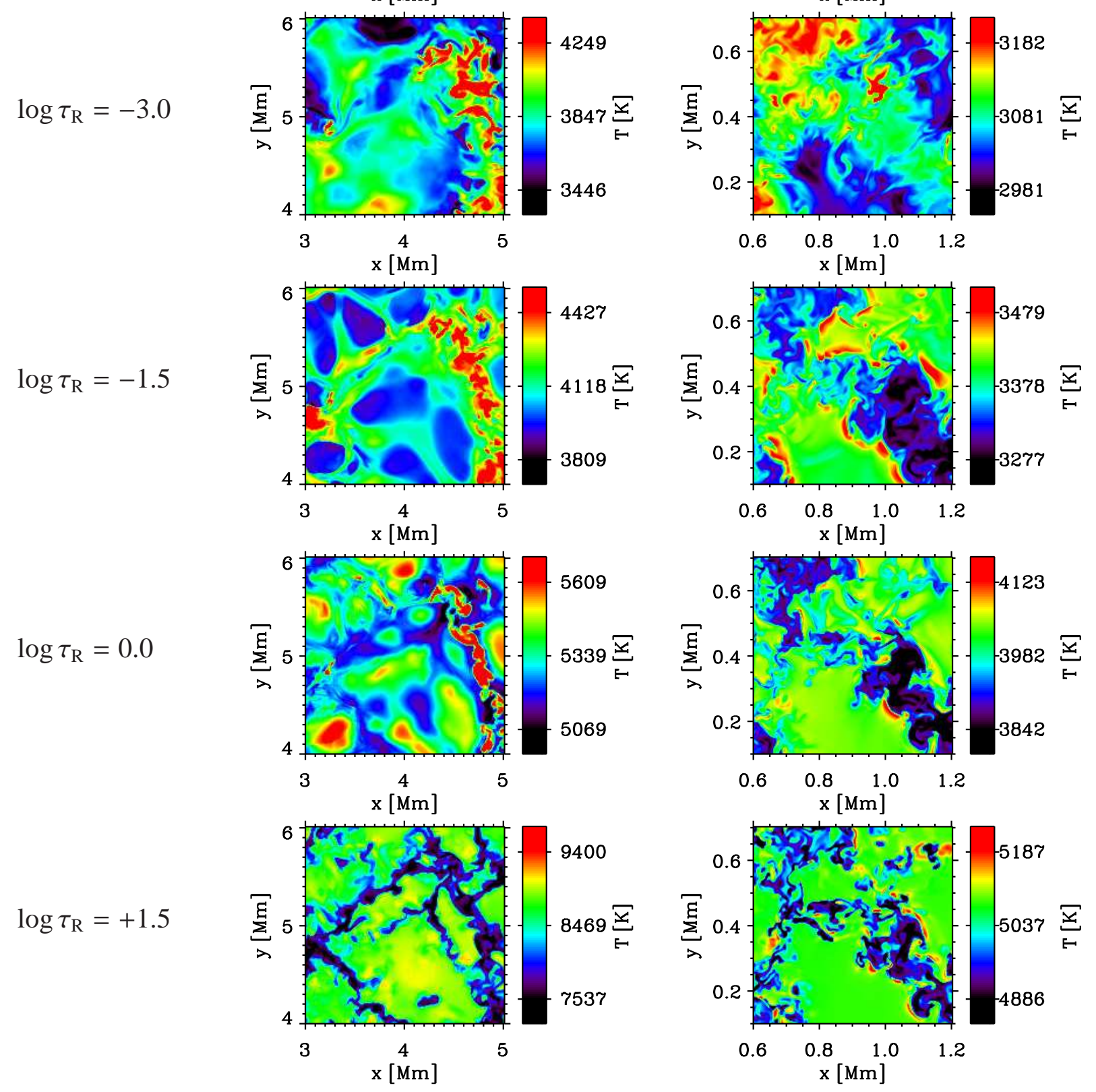

Figure 3.40: Maps of the temperature, $T$, on iso- $\tau_{\mathrm{R}}$ surfaces of a region from the K0V simulation with $B_{0}=100 \mathrm{G}$ (left column) and from the M2V simulation with $B_{0}=500 \mathrm{G}$ (right column). 

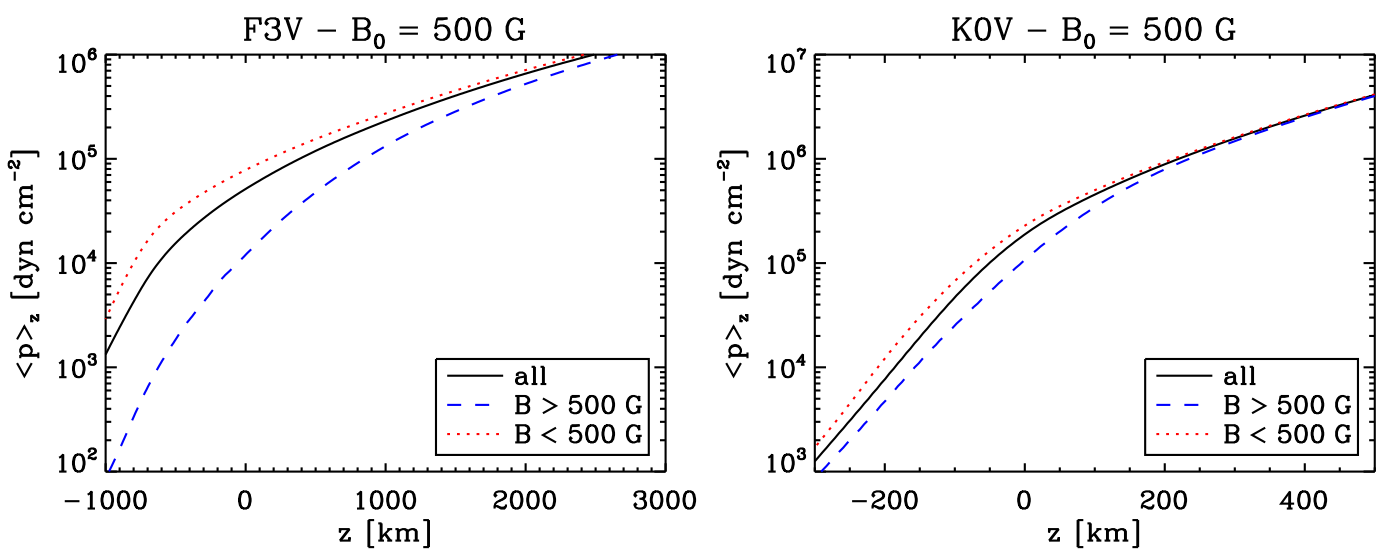

Figure 3.41: Gas pressure averaged over horizontal planes in the F3V (left panel) and K0V simulations (right panel) with $B_{0}=500 \mathrm{G}$. The blue dashed curves shows averages within magnetic flux concentrations $(B>500 \mathrm{G})$, the red dotted curve in the surroundings $(B<500 \mathrm{G})$, the black solid curve gives the averages over the total area.

heated radiatively through their side walls near the optical surface. Fig. 3.39 suggests that this even works to some extent in small dark magnetic structures (micropores), which are hotter than the non-magnetic downflows at $\log \tau_{\mathrm{R}}<-1.5$. This mechanism does not work efficiently in the shallow depressions caused by the magnetic field on $\mathrm{M}$ dwarfs (cf. Figs. 3.27). Consequently, micropores on $M$ stars are cool except for the small-scaled hot features at their edges. However, there is some heat generated by viscous and ohmic heating in the upper atmosphere $\left(\log \tau_{\mathrm{R}} \approx-3\right.$; cf. Fig. 3.35).

\subsubsection{Pressure and density}

Figure 3.41 shows the gas pressure averaged over horizontal planes within flux concentrations $(B>500 \mathrm{G})$ and in the surroundings $(B<500 \mathrm{G})$ for the $500 \mathrm{G}$ runs of the F3V and K0V simulations. Owing to the high magnetic pressure inside the flux concentrations, the gas pressure is strongly reduced. At constant geometrical depth, this effect is most strongly pronounced in the F3V simulation, where the gas pressure reduction is on average around $85 \%$ at the optical surface (locally it can surpass $90 \%$ ). The gas pressure reduction becomes smaller for the cooler stars because the field strength at the optical surface are similar while the photospheric gas pressure increases by about one order of magnitude (cf. Fig. 3.7). In the coolest model, the M2V star, it amounts to roughly $25 \%$ at $z=0$. This is consistent with a smaller reduction of the density in the cooler models, which entails much shallower Wilson depressions (cf. Fig. 3.27).

As the absorption coefficient is highly pressure-dependent in the photospheres (approximately $\kappa \varrho \propto p^{2}$, see discussion in Sect. 3.3.1), the effect of the magnetic field on $\langle p\rangle_{\tau}$ is quantitatively and qualitatively different from on $\langle p\rangle_{z}$. Figure 3.42 shows the difference in $\langle p\rangle_{\tau}$ between simulations with $B_{0}=500 \mathrm{G}$ and their non-magnetic counterparts separately for up- and downflows. As for the temperature, this difference is relatively similar in quality and quantity in up- and downflows, although almost the entire magnetic flux is concentrated in downflows. The average gas pressure on a given iso- $\tau_{R}$ surface 

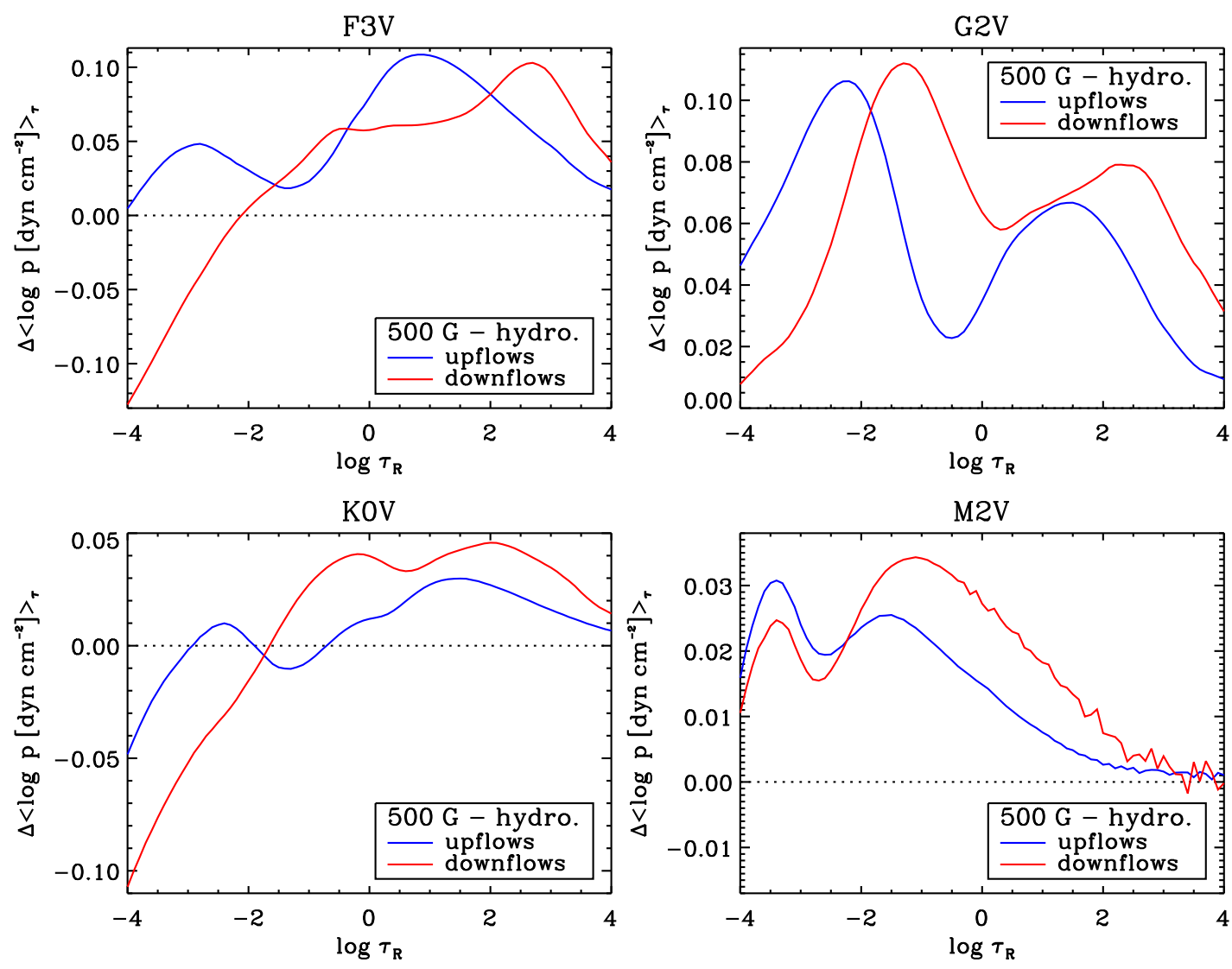

Figure 3.42: Differences in gas pressure averaged on iso- $\tau_{R}$ surfaces between the $500 \mathrm{G}$ runs and the non-magnetic runs separately for up- and downflows.

is higher in the magnetic runs over large parts of the given depth range. As the area fraction of the (partially evacuated) low- $\beta$ regions increases with height (cf. Fig. 3.20), the average gas pressure drops more quickly with height in the $500 \mathrm{G}$ runs than in the non-magnetic runs, entailing a steeper gradient of $\kappa \varrho$. Neglecting the effect of the temperature on $\kappa$, this would result in a somewhat larger horizontally averaged $\kappa \rho$ and thus larger average gas pressure at any given optical depth. The highest pressure is found in the periphery of magnetic structures (see Fig. B.15 in Appendix B), which is expected because these are viewed through a partially evacuated and consequently very transparent magnetic structure: the optical depth is already reduced at these points, while pressure and density are not (cf. Fig. 3.27). At $\log \tau_{\mathrm{R}}<-2$, however, the gas pressure often becomes smaller in the $500 \mathrm{G}$ runs than in the non-magnetic runs (especially in the downflows). This is related to the higher temperature in the magnetic downflow regions in the high atmosphere (cf. Fig. 3.40): a higher temperature entails a higher opacity (see Fig. B.17 in Appendix B), which moves the iso- $\tau_{R}$ surfaces upwards to lower pressures and densities. In fact, the lowest pressure values on iso- $\tau_{R}$ surfaces are observed in hot magnetic structures (cf. Fig.B.15 in Appendix B).

Figure 3.43 shows the relative differences of the gas pressure at the optical surface, $p_{0}:=\left\langle p\left(\tau_{\mathrm{R}}=1\right)\right\rangle$, between the magnetic simulations and their non-magnetic counterpart. 


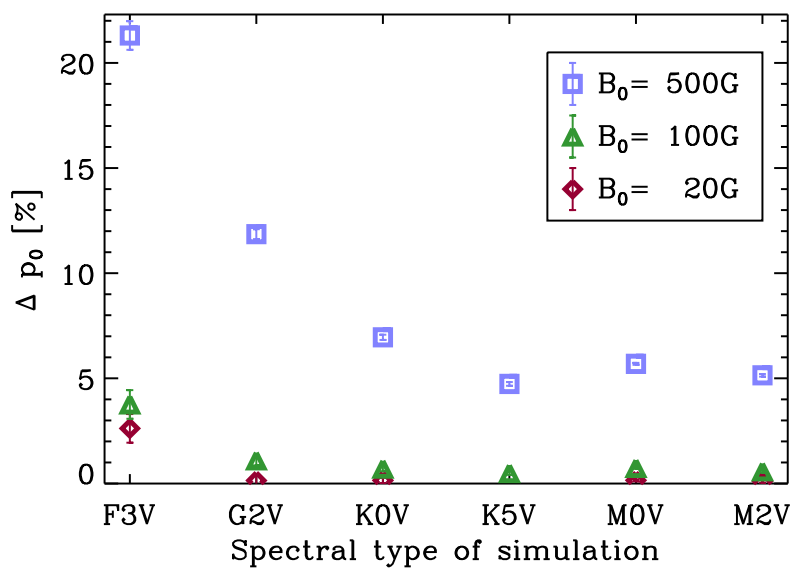

Figure 3.43: Relative increase of $p_{0}$, the pressure at the optical surface, in the magnetic runs in comparison to their non-magnetic counterparts.

Generally, the gas pressure at the optical surface is higher in the magnetic runs. The strongest effect is visible in the $500 \mathrm{G}$ runs $(\sim 5-7 \%$ difference to non-magnetic run in the $\mathrm{M}$ - and K-star simulations, $12 \%$ in the G2V simulation and $22 \%$ in the F3V simulation).

The density shows a behaviour very similar to that of the gas pressure (see Figs. B.16 and B.18 in Appendix B). This is because the relative temperature fluctuations on surfaces of constant optical depth are generally smaller than the relative pressure fluctuations. In the temperature range of interest $(3000-7000 \mathrm{~K})$ the mean molecular weight does not change significantly, because neither molecule formation nor hydrogen ionisation play an important role in this range. Therefore, the ratio of gas pressure and density only depends linearly on temperature and is thus not strongly varying within the photosphere.

In contrast to the temperature, the impact of the pressure (and density) on observationally detectable signals (i.e. on spectral lines) is relatively weak ("pressure broadening"). Although the magnetic field affects the pressure and density very strongly on horizontal planes and still considerably on surfaces of constant optical depth, the direct effect of this modification on spectral lines is most likely undetectable for the considered spectral types and values of $B_{0}$. However, the effect of the magnetic field on optical depth (see Sect. 3.3.1) and temperature (see Sect. 3.3.3.1) are mainly consequences of its effect on the gas pressure.

\subsubsection{Fluctuations of temperature and pressure}

Figure 3.44 shows the rms fluctuations of gas pressure and temperature for the F3V and $\mathrm{M} 2 \mathrm{~V}$ simulations on surfaces of constant optical depth. Both quantities change considerably from non-magnetic to magnetic simulations. The pressure fluctuations become much larger with magnetic field above the optical surface in all stars. Below the optical surface the effect is smaller, but in most cases there is still a considerable increase. The reason for this strong increase is the combination of higher pressure in the periphery of the magnetic flux concentrations(cf. Fig. B.15) and the considerably changed atmosphere structure within the flux concentrations. The high pressure in the periphery is a geometrical effect 

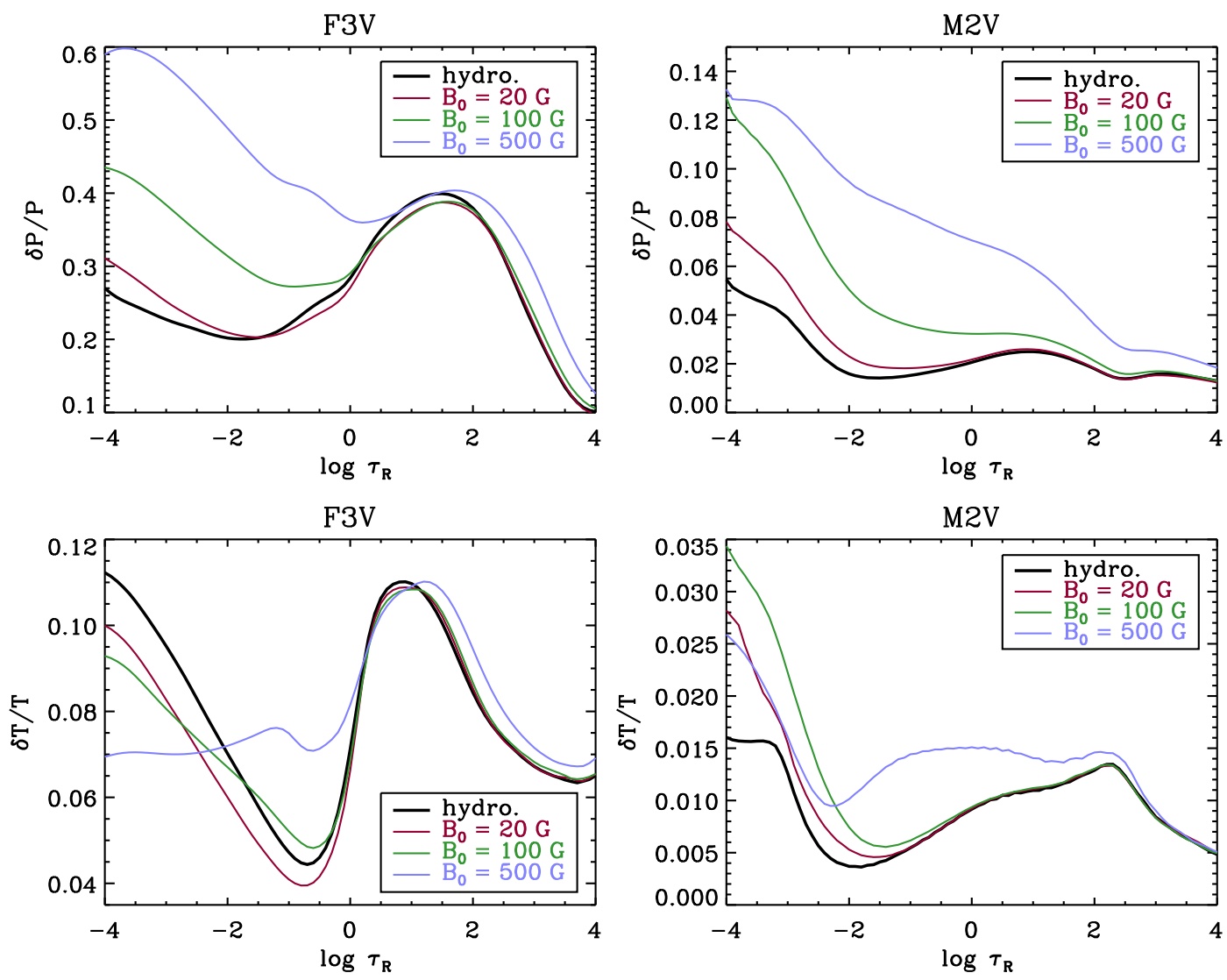

Figure 3.44: Profiles of the relative rms fluctuations of the gas pressure (upper panels) and the temperature (lower panels) on iso- $\tau_{\mathrm{R}}$ surfaces for the F3V (left) and $\mathrm{M} 2 \mathrm{~V}$ (right) simulations. The curves for the non-magnetic simulation runs (hydro.) correspond to the curves in Fig. 3.11.

of the inclined walls of the magnetic flux concentrations (cf. Fig. 3.27), which affect the optical depth structure and shift surfaces of constant optical depth downwards in the periphery of the magnetic flux concentrations. Within the flux concentrations, the opacity is either enhanced (hot structures) or reduced (cool structures) leading to a reduced or enhanced gas pressure, respectively.

The effect on the temperature fluctuations is weaker and mostly confined to $\log \tau_{\mathrm{R}} \lesssim$ 2. For the hotter stars, the temperature fluctuations even become smaller in the higher photosphere with magnetic field, while the M-star simulations show somewhat enhanced temperature fluctuations in the magnetic runs. On the one hand, the viscous heating in shocks, which only occur in the hotter stars, becomes less with magnetic field. On the other hand, Ohmic heating and viscous dissipation of vortices and magnetohydrodynamic waves are heat sources which lead to temperature inhomogeneities. While the former effect leads to a reduction of the temperature fluctuations caused by the magnetic field in the hotter stars, the latter leads to an increase of the temperature fluctuations in the cooler stars. 

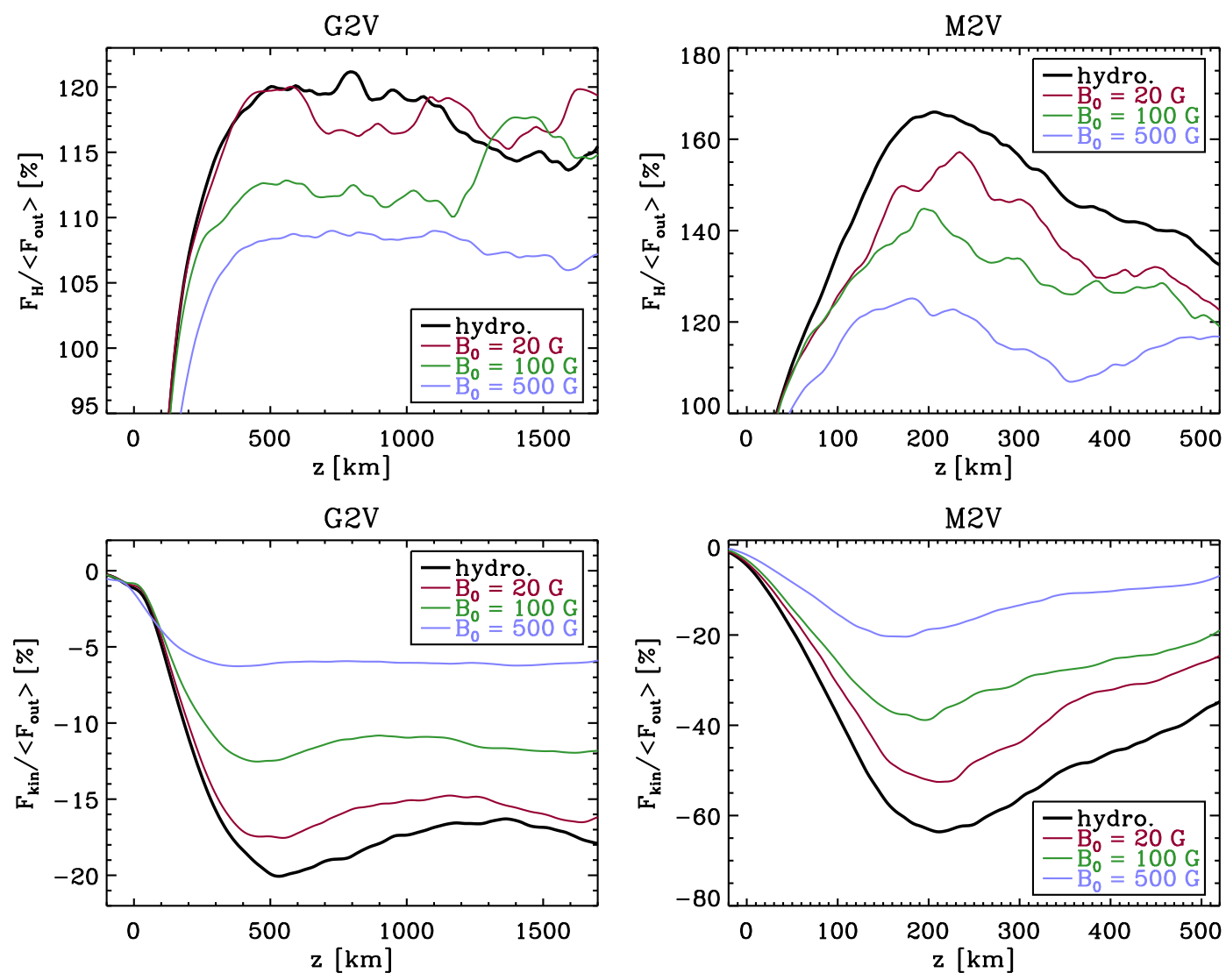

Figure 3.45: Enthalpy flux (top) and kinetic energy flux (bottom) in the G2V and M2V simulations. Both quantities are given in units of the total energy flux (cf. Fig 3.12).

\subsubsection{Energy flux}

As shown in Sect. 3.1.4, the energy flux in the convective envelope is composed of two fluxes of opposite sign. In the non-magnetic case, there is a considerable kinetic energy flux from the surface towards the stellar interior of -20 to $-60 \%$ percent of the total energy flux. The net enthalpy flux is directed upwards and carries more than $100 \%$ of the total flux to compensate the negative net kinetic energy flux. Figure 3.45 gives the depth profiles of the enthalpy flux and kinetic energy flux of the G2V and M2V stars with different $B_{0}$. The other spectral types show qualitatively similar results. The kinetic energy flux is reduced in all magnetic runs. In the $500 \mathrm{G}$ runs, it is less than a third of its value without magnetic field. This is mainly a consequence of the strong concentration of magnetic flux in downflows, which entails a lower downflow speed and (see Sect. 3.3.2) and lower density in downflows (see Sect. 3.3.3.2). The enthalpy flux is thus mostly smaller in the magnetic runs. The presence of the magnetic field reduces the enthalpy flux in upand downflows. However, the reduction in the upflows is somewhat stronger than in the downflows, causing the difference in the net enthalpy flux. In greater (geometical) depth, enthalpy flux and kinetic energy flux do not seem to add up to exactly $100 \%$ in the plots of Fig. 3.45. This is probably due to the low number (six) of snapshots used for this analysis and to the fact that the system has not reached a global equilibrium again, as the time 
since the injection of the magnetic field is short compared to the Kelvin-Helmholtz time. 



\section{Granulation}

\subsection{Granule segmentation ${ }^{1}$}

As a basis for the analysis of the granulation patterns, we used a time series of 500 synthetic (bolometric) intensity images for each of the six simulation runs (for details on the simulations considered, see Sect. 2.3.2). The images are separated by $20 \delta t$ where $\delta t$ is the simulation time step (depending on the star, $2.9 \mathrm{~s} \leq 20 \delta t \leq 4.3 \mathrm{~s}$ ), which is well below the typical lifetime of the stellar granules (of the order of several minutes).

We implemented a granule segmentation and tracking algorithm, which was designed to follow granules in intensity images. In order to scale to the different intensity values of the stars the algorithm uses the normalised (bolometric) intensity fluctuation,

$$
\hat{I}=(I-\langle I\rangle) / \sigma_{I}, \quad \text { with } \sigma_{I}=\sqrt{\left\langle I^{2}\right\rangle-\langle I\rangle^{2}},
$$

where $\langle\ldots\rangle$ denotes the temporal and spatial mean. This normalisation simplifies the comparison between stars with different surface temperatures and temperature contrasts.

In the first step, $S 1$, of the algorithm, the image is divided into sub-images of $2 \times 2$ pixels. For each sub-image $i$, the mean, $\langle\hat{I}\rangle_{i}$, and the standard deviation, $\sigma_{i}(\hat{I})$, of the normalised intensity contrast are calculated. The algorithm then utilises the fact that granules are extended bright features, while intergranular lanes are dark and rather sharp (i.e. the intensity gradient is large). Accordingly, depending on its values of $\langle\hat{I}\rangle_{i}$ and $\sigma_{i}(\hat{I})$, each sub-image is put into four categories, characterised by a number $a$ between 0 and 1 . A high value of $a$ means that the probability that the sub-image is part of a granule is high. The categories are defined as:

$$
a=\left\{\begin{array}{rl}
0, & \text { if }\langle\hat{I}\rangle_{i}<I_{\text {inter }} \text { or } \sigma_{i}(\hat{I})>\sigma_{h} \\
0.3, & \text { if } I_{\text {inter }} \leq\langle\hat{I}\rangle_{i} \leq I_{\text {gran }} \text { and } \sigma_{h}>\sigma_{i}(\hat{I}) \geq \sigma_{m} \\
0.7, & \text { if } I_{\text {inter }} \leq\langle\hat{I}\rangle_{i} \leq I_{\text {gran }} \text { and } \sigma_{m}>\sigma_{i}(\hat{I}) \geq \sigma_{l} \\
1, & \text { if }\langle\hat{I}\rangle_{i}>I_{\text {gran }} \text { or } \sigma_{i}(\hat{I})<\sigma_{l}
\end{array} .\right.
$$

The threshold values $I_{\text {gran }}, I_{\text {inter }}, \sigma_{l}, \sigma_{m}$, and $\sigma_{h}$ enter the segmentation as parameters.

The "map" resulting from this categorising (cf. upper right panel of Fig. 4.1) has half the resolution of the original image. In the next step, $S 2$, this map is smoothed by a Gaussian ( $\sigma=2$ pixels), interpolated back onto the original grid size, and clipped at a threshold value of typically 0.5 . The continuous regions obtained in this step already roughly correspond to granules in the original images but are usually somewhat smaller. In the last step, $S 3$, using a multiple-level tracking (MLT) method (cf. Bovelet and Wiehr

\footnotetext{
${ }^{1}$ This section has been published as Sect. 3.1 in Beeck et al. (2013b)
} 


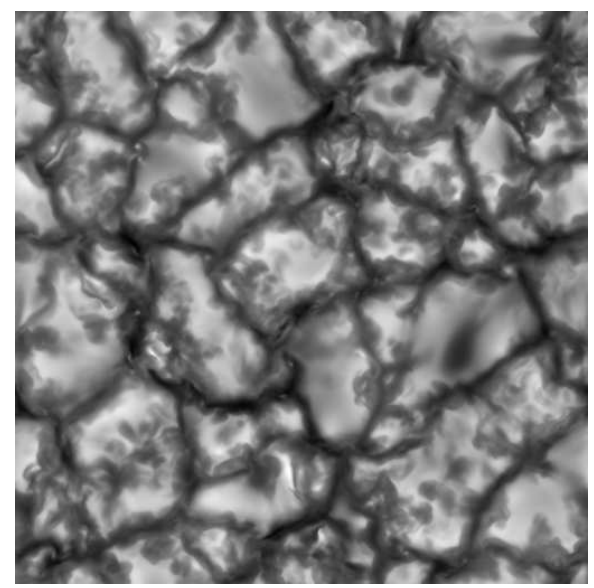

$(S 1)$

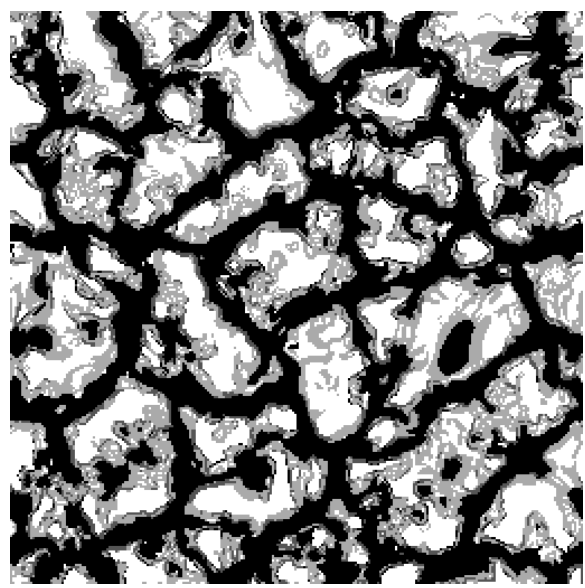

$(S 2)$

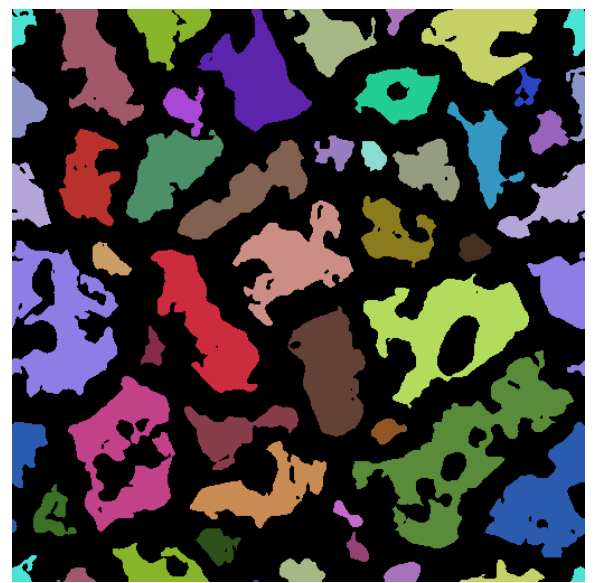

$(S 3)$

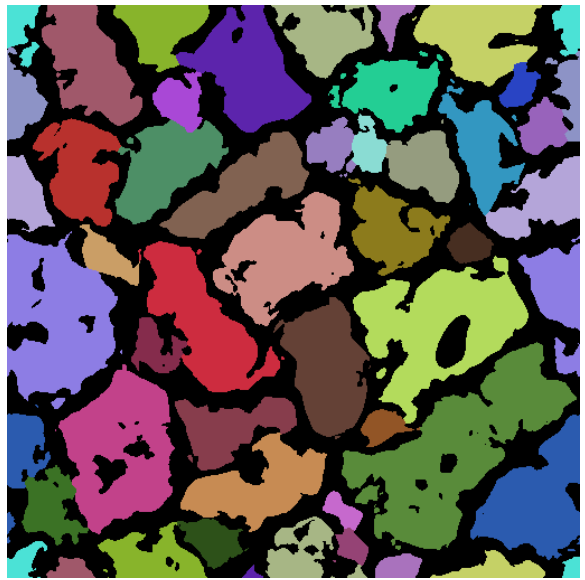

$(T 1)$
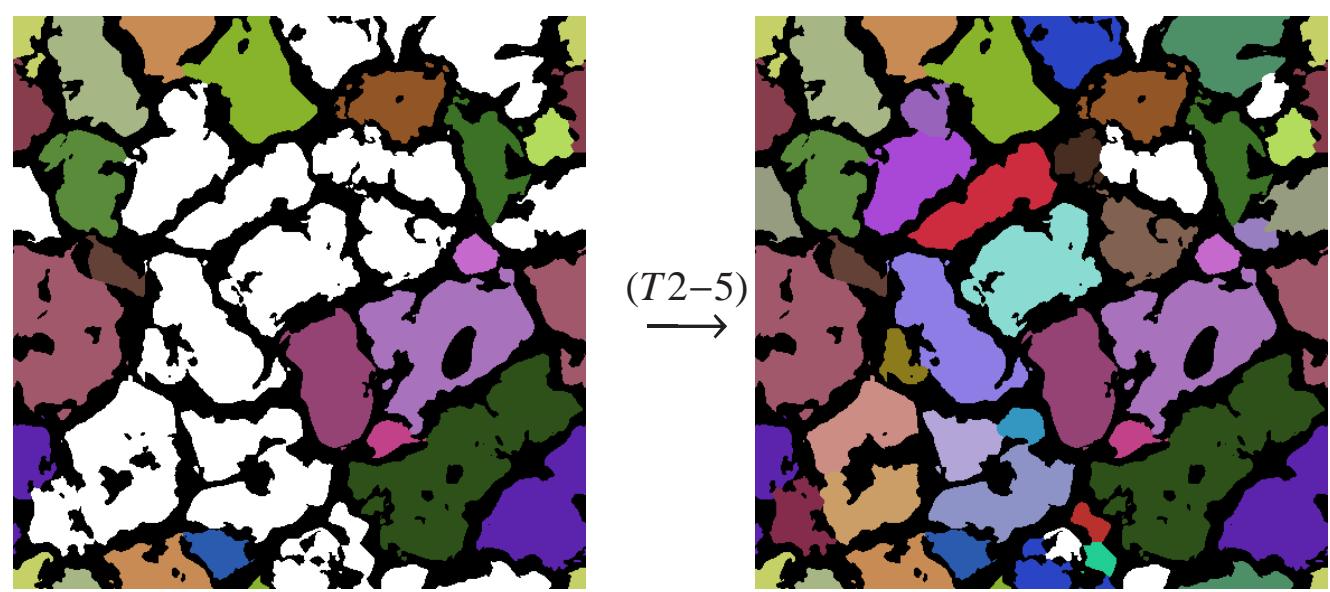

Figure 4.1: Illustration of the segmentation and tracking algorithm (snapshot of the G2V simulation). Top panels: raw intensity image (left); map of sub-image categories (the number $a$ is shown in grey scale; right). Middle panels: granule segmentation before the multiple-level tracking (MLT; coloured areas = granules; black = intergranular lanes; colours were chosen randomly and for clarity; left); segmented image after MLT (right). Bottom panels: trackable (coloured) and non-trackable (white) granules after the first segmentation/tracking run (left) and after the fifth run (right). 
2001), all pixels with $\hat{I}>I_{\text {inter }}$ which are not yet part of a detected granule are either assigned to one of the granules already detected or considered as small-scale intergranular structures, which were excluded with a minimum-size criterion. The first four images of the series shown in Figure 4.1 give an illustration of the segmentation algorithm.

For a statistical analysis of granule properties (such as size, mean intensity, etc.) in snapshots this algorithm provides a suitable basis. However, it is not sufficient for tracking granules in time since there are often cases where the distinction between a single granule with substructure and a group of two or more granules is not clear. The interpretation of such cases by the algorithm could fluctuate from snapshot to snapshot, which would give rise to numerous granules which are identified only in one snapshot. To overcome this problem and enable tracking granules, a modification of the scheme is necessary. First, the series of images were subdivided into packages of 10 sequential images. After running the segmentation algorithm described above for all 10 images, a simple comparison of overlapping granule area in subsequent images yields a first tracking step (T1). Structures which exist through the whole sub-series of images are considered as unambiguous cases of trackable ("stable") granules. Next, the segmentation is run again only considering the parts of the 10 images where no trackable granules have been detected in the previous step. A slight variation of the segmentation parameters enables the algorithm to find further granules in this run. After about five iterations $80-90 \%$ of the granules can be tracked through the series of 10 subsequent images. The remaining granules are probably in a phase of evolution where their definition is particularly difficult (splitting or merging). The last two images of the series shown in Figure 4.1 show the trackable granules after the first segmentation/tracking run and after the last (fifth) run, respectively. Most of the granules are detected by the algorithm. There are, however, a few untrackable granules: the largest white region appearing in the lower right panel of Figure 4.1, for instance, seems to belong to a larger granule-like, bright area. A closer inspection of the original image and the intermediate steps of the algorithm suggests, however, that this larger area is composed of three parts. While the middle and the right part do not appear clearly divided from each other by a dark lane, there is a lane-like structure between the left-hand side part of the area and the rest. The algorithm will therefore find this left-hand side part as one granule living through the short sub-series of 10 images for most parameter sets whereas the rest of this granule-like area will sometimes appear as two, sometimes as one detected granule and remain untrackable.

Finally, the packages are merged and granules which exist in subsequent packages are identified (search for overlapping area, similar in size). The described procedure efficiently finds long-lived granules, but it also limits the lifetime resolution, since, by definition in this algorithm, the granules can only have lifetimes which are integer multiples of 10 subsequent images. This corresponds to a minimum lifetime of roughly 29 to $43 \mathrm{~s}$, depending on the simulated star.

In order to use the same data set and algorithm for the statistical analysis (Sect. 4.2.3) of snapshots as for the evolution and lifetime analysis of the granules (Sect. 4.2.4), we limited ourselves to a rather short sequence of 500 images spanning roughly half an hour of stellar time. As a good compromise between statistical independence and statistical significance, we considered every $15^{\text {th }}$ image for the statistical part of the analysis, which corresponds to a time step of the order of one minute of stellar time. Since this is already smaller than the typical evolution time scale of the granules ( several minutes), a finer 
sampling would not improve the statistical significance. For the statistical analysis in Sect. 4.2.3, all granules (trackable and not trackable) were considered, whereas for the determination of the lifetime and time averaged properties, the untrackable granules were excluded from the sample.

\subsection{Properties of the granulation in the non-magnetic sim- ulations $^{2}$}

\subsubsection{General appearance of the granulation pattern}

All six simulations considered here show spatial patterns in the vertically emerging (bolometric) intensity reminiscent of solar granulation (see Fig. 3.1). The visual appearance indicates a qualitative change of the convection within the model sequence around spectral type $\mathrm{K}$, which will be analysed further in this section.

The top panel of Figure 4.2 shows histograms of the bolometric intensity, illustrating the difference of about a factor of ten between the average intensity of the hottest and the coolest model, as well as the decreasing intensity contrast (width of the distribution). In what follows, we use the normalised intensity fluctuation $\hat{I}$ as defined in Eq. (4.1). For this quantity, the differences in mean intensity and contrast are removed by the normalisation.

The middle and bottom panel of Figure 4.2 show histograms of the normalised intensity fluctuations, $\hat{I}$, (solid curves) calculated from a large number of statistically independent snapshots. In all cases, the distributions show signs of bimodality, i. e. there is a bright and a dark component. Without taking into account possible asymmetries of the two individual components, the balance between them varies along the model sequence: the brighter component gets relatively weaker from $\mathrm{F} 3 \mathrm{~V}$ to $\mathrm{K} 0 \mathrm{~V}$ and then stronger again from K0V to M2V.

The dashed and dotted curves in Figure 4.2 show histograms of separate distributions of $\hat{I}$ for intergranular lanes and granules, respectively, as defined by our segmentation algorithm. The fractional area covered by granules is almost invariant between simulations, which effectively couples the width and amplitudes of the granular and intergranular components of the intensity distributions. The intensity distribution of the granular component is broader and has a lower amplitude in the three hotter models than in the cooler ones. It becomes more asymmetric from F3V to K0V with its peak shifting towards lower intensities. From K0V to M2V, the granular component becomes more symmetrical again and somewhat narrower. In contrast, the intergranular component is relatively narrow and has a high amplitude in the hottest models and becomes broader in the K- and M-star simulations. Consequently, along the model sequence, the granular component becomes weaker in the combined distributions from $\mathrm{F} 3 \mathrm{~V}$ to $\mathrm{K} 0 \mathrm{~V}$ and than stronger from $\mathrm{K} 0 \mathrm{~V}$ to $\mathrm{M} 2 \mathrm{~V}$. In the M-star simulations, the intergranular component merges with the flank of bright component, which results in an apparently unimodal distribution with a peak at the typical granule intensity. The decreasing intensity contrast $\sigma_{I} /\langle I\rangle$ with decreasing effective temperature is a consequence of the lower energy flux $\left(\propto T_{\mathrm{eff}}^{4}\right)$ and higher density of the emitting layers. Higher density results in a higher heat capacity per volume which means

\footnotetext{
${ }^{2}$ This section has been published as Sect. $3.2-3.5$ in Beeck et al. (2013b).
} 

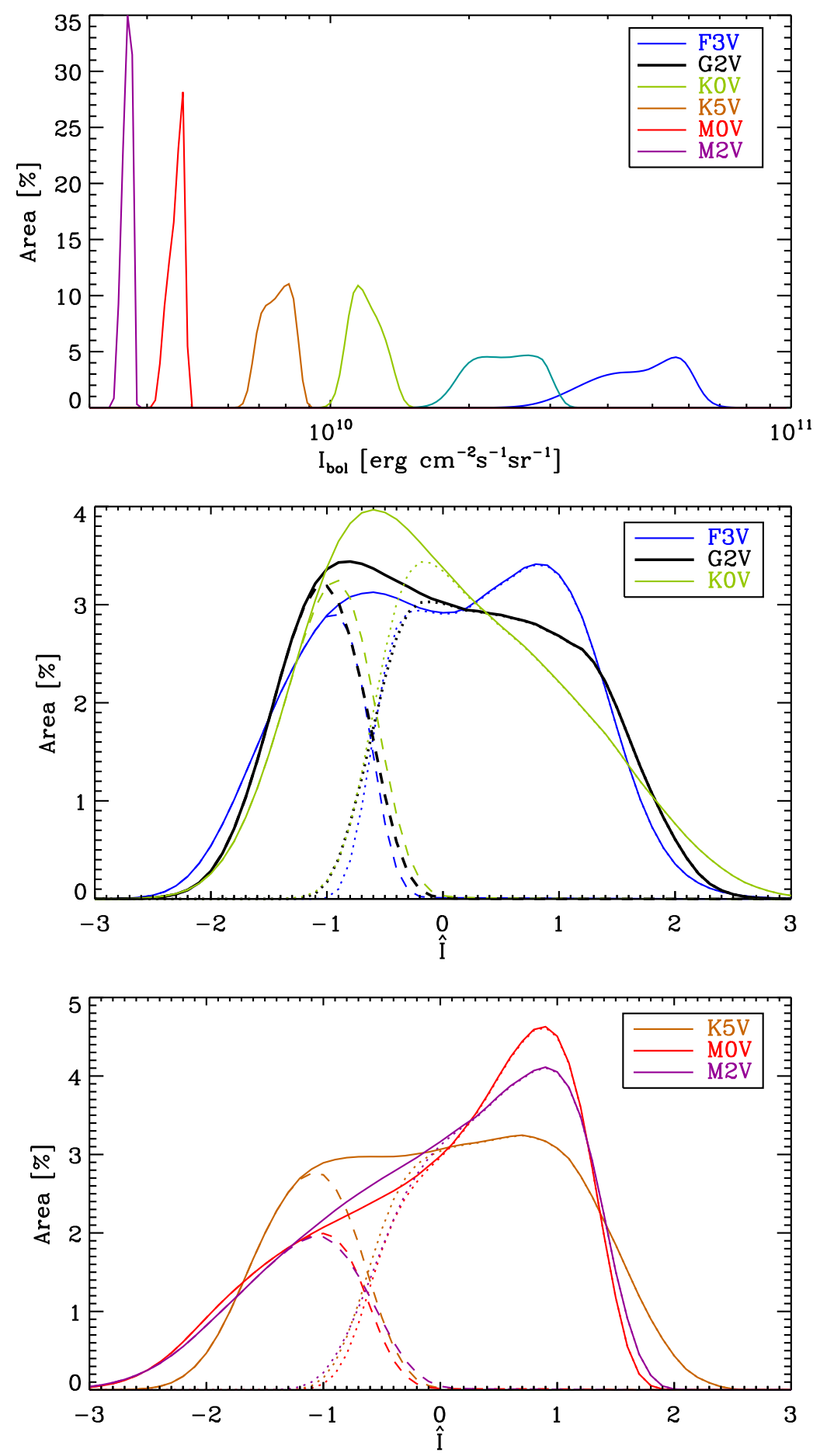

Figure 4.2: Intensity histograms. Top panel: histograms of the bolometric intensity. The bins are logarithmically equidistant with a bin size of $0.01 \mathrm{dex}$. Middle and bottom panels: histograms of the normalised intensity fluctuation $\hat{I}$ (see Eq. (4.1)). The bins are linearly equidistant with a bin size of 0.1. Solid curves show the histograms of the entire area, dotted and dashed curves in the middle and bottom panel show the histograms of granules and intergranular lanes, respectively, as detected by the algorithm described in Sect. 4.1. 
that the enthalpy flux can be sustained by a smaller temperature contrast between up- and downflows (see Sect. 3.1.3). The smooth appearance and lack of bright sub-structure in $\mathrm{K}$ - and M-star granules can be explained by the veiling of the granulation by an optically thick layer (cf. Nordlund and Dravins 1990a): for decreasing effective temperature, the transition from convective to radiative heat transport occurs over a greater range of pressure scale heights and sets in at greater optical depth. This leads to a thin subsurface layer in which diffusive radiative energy transport plays an important role. In most cases, the top part of this layer (the region around $\tau_{\mathrm{R}} \approx 1$ ) is stable against convection. The vertical motions which are present in this layer mainly represent overshoot from the unstable layers below so that the correlation between upflow velocity and temperature is less pronounced than in the convection zone. The granules of cooler stars are veiled by this optically thick convectively stable layer, which brakes convective flows and smears out the inhomogeneities in the temperature of the upflows by horizontal radiative diffusion.

Compared to the these "veiled" granules, granules of G- and F-type stars are "naked" in the sense that the bulk of the energy is carried by convection up to the height where the atmosphere becomes optically thin. The transition to radiative energy transport occurs very rapidly owing to the strong temperature dependence of the opacity in the temperature range in which the cooling sets in. The corresponding layer is an order of magnitude smaller than the typical granule diameter. The cool downflows, however, remain optically thin into deeper layers and over a larger depth range. This leads to a strong corrugation of the optical surface. Together with the high (sometimes super-sonic) flow velocities, which entail strong deviations from hydrostatic equilibrium, this makes the 3D structure of F- and G-type stars more complex than that of cooler stars.

Figure 4.3 illustrates the differences between the "naked" granulation of the F3V star with much brightness sub-structure, strong deviations from hydrostatic equilibrium, a complex velocity field, and a corrugated optical surface on the one hand and the "veiled" granulation of the K5V star with a lack of brightness sub-structure, small deviations from hydrostatic equilibrium, a less complex velocity field, and a rather flat optical surface on the other hand (see also Fig. A.1).

\subsubsection{Vortex motions}

Horizontal and vertical vortex flows of (sub-)granular scale have been detected in highresolution solar observations (e.g. Bonet et al. 2008, 2010, Steiner et al. 2010, Vargas Domínguez et al. 2011, Wedemeyer-Böhm et al. 2012) and also in solar simulations (e. g. Stein and Nordlund 1998, Moll et al. 2011). Ludwig et al. (2006) reported swirling downflows also in simulations of cool main-sequence stars of spectral type M.

We find vertical and horizontal vortex motions in all our simulations. Horizontal vortices are usually located along the edges of granules, where they are driven by the shear between the horizontal outflow from the granule centres and the more randomly oriented flows in the optically thin layers above. Vertical vortices occur in strong downdrafts as consequence of angular momentum conservation in the highly stratified medium. Strong vertical vortices are partially evacuated owing to the centrifugal force, which leads to a local depression of the optical surface. Analogous to small-scale bright magnetic features (magnetic bright points), for which the depressions of the optical surface are caused by the magnetic pressure, the vortex flows sometimes can appear as features of enhanced 
F3V
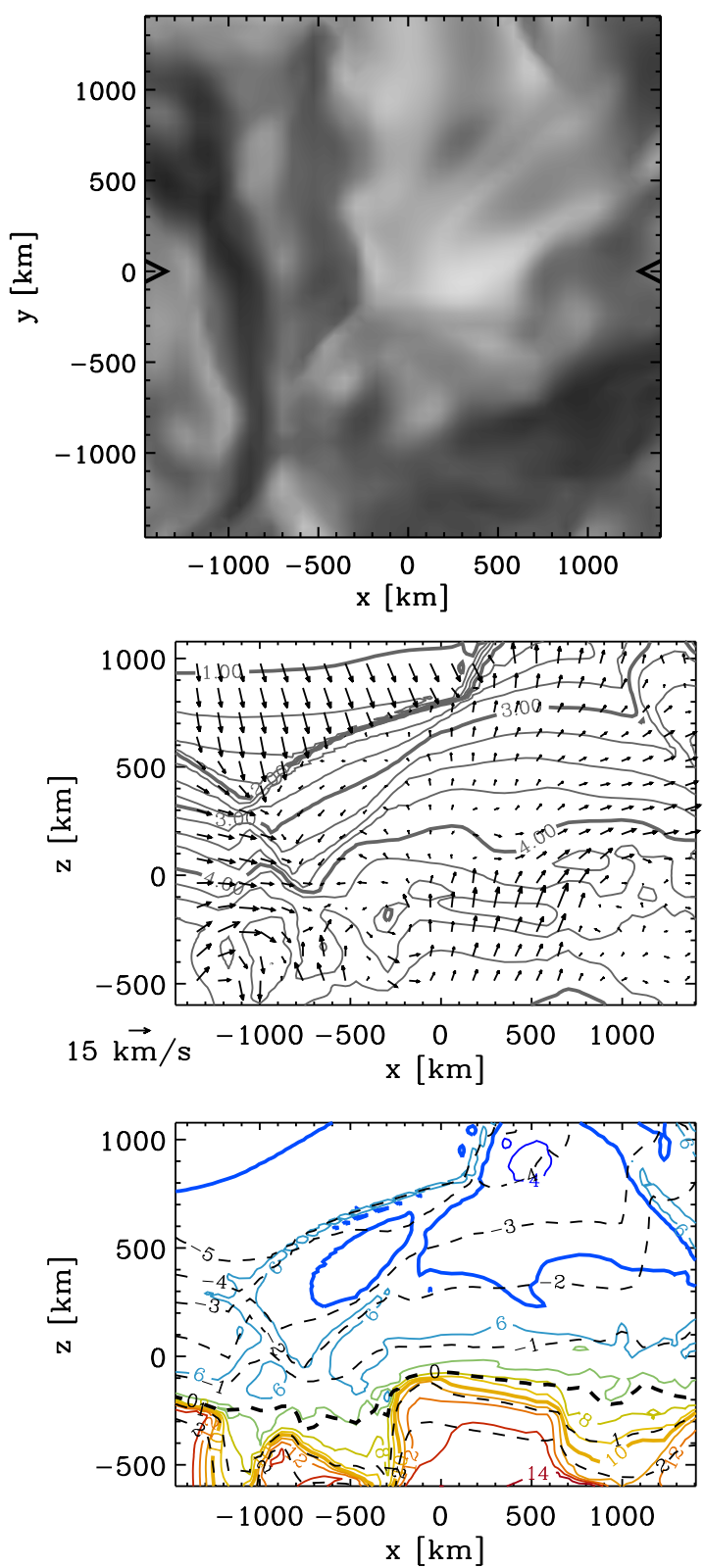

K5V
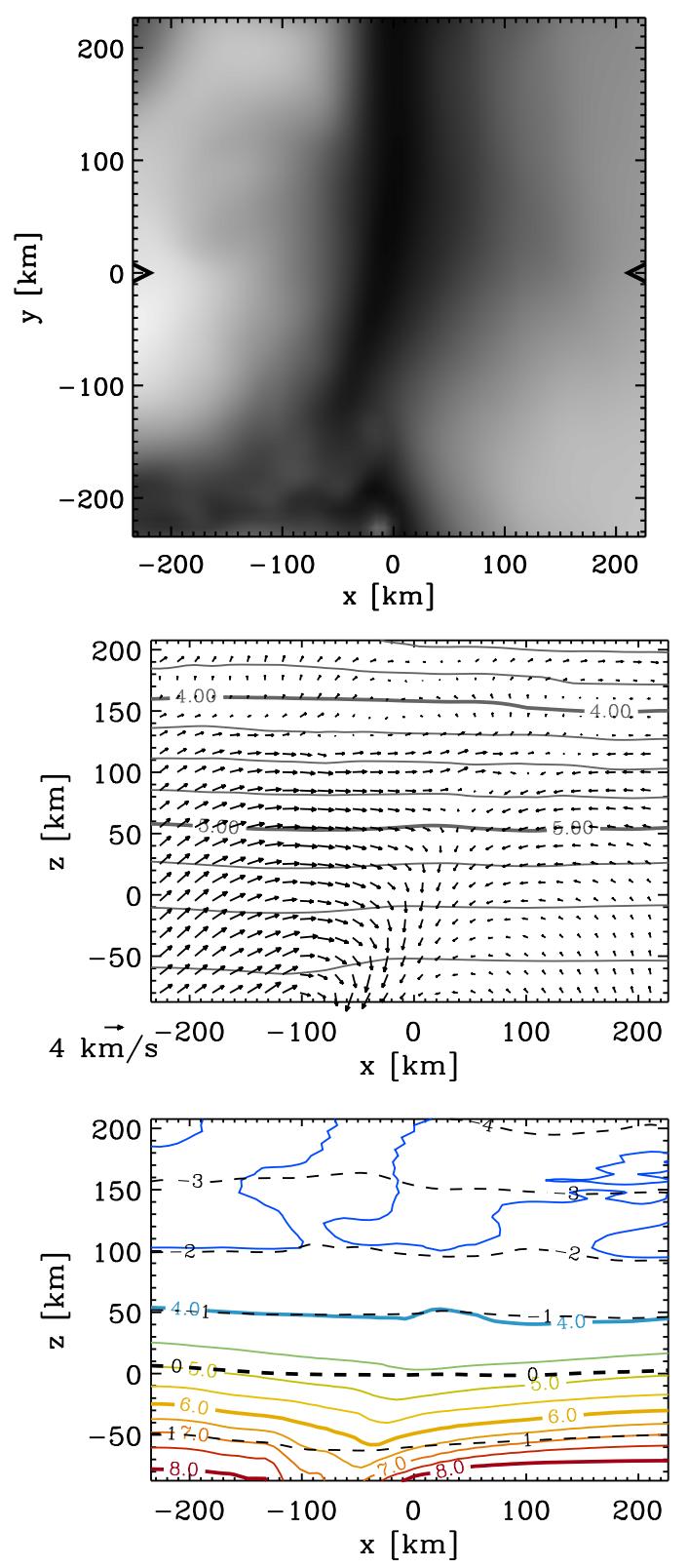

Figure 4.3: Comparison between F3V and K5V granulation. Top panels: Images of the bolometric intensity in small areas of simulation snapshots corresponding to about the size of one granule in the respective simulations. The images are 50 and 58 grid points across, for the $\mathrm{F} 3 \mathrm{~V}$ and $\mathrm{K} 5 \mathrm{~V}$ simulations, respectively. The black triangles mark the position $y=0$ of the vertical cuts shown in the lower panels. Middle panels: Vertical cuts through the near-surface layers; arrows indicate the velocity field, grey contours show the isobars (labels give the logarithm of the pressure in $\mathrm{dyn}_{\mathrm{cm}} \mathrm{cm}^{-2}$ ). Bottom panels: Same cuts as middle panels showing the temperature field (coloured solid contours, labels in $1000 \mathrm{~K}$ ) and levels of constant Rosseland optical depth $\tau_{\mathrm{R}}$ (dashed contours, labels give logarithm of $\tau_{\mathrm{R}}$ ). 
intensity due to side-wall heating of the interior of the depression. The vortices in solar MURaM simulations are described in detail by Moll et al. (2011, 2012).

The formation of bright vortex features requires a sufficiently high spatial resolution of the simulation. In our models, the horizontal resolution scales with the granule size and the vertical resolution with the local pressure scale height (see Sect. 3.3.2) in order to be dynamically comparable to each other. We observe bright vortices to be most common in the K-star simulations (especially K0V). This is probably an opacity effect. Generally, the opacity, $\kappa(p, T)$, depends on pressure and temperature. However, at pressures around $10^{-4}$

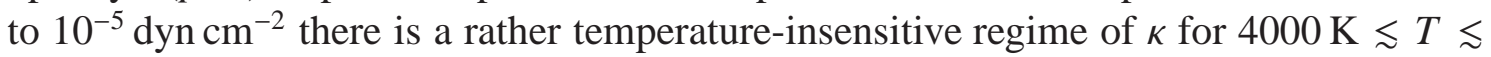
$5000 \mathrm{~K}$. The absorption coefficient, $\kappa \varrho$, thus mainly depends on density. Photospheric transitions in this temperature range are not accompanied by the usual strong gradient of $\kappa \varrho$. This is the case for the intergranular lanes of the $\mathrm{K}$ stars. Therefore, the location of the photosphere, in these cases, is more sensitive to density than to temperature. The partial evacuation caused by vertical vortices in K stars locally moves the photosphere inward to higher temperatures, entailing a brightening of the cores of these vortices. In the other stars, $\kappa \varrho$ is more sensitive to temperature and the depth of the local photosphere in most vortices only changes little and the brightening is much less pronounced.

Figure 4.4 illustrates the vertical vortices in the K5V simulation. In the left panel, a snapshot with eight sharp intensity maxima above $\hat{I}>1.0$ is shown. All these features are associated with vortices, but there are many weaker intensity structures in this image which are also linked to vortex motion. The bottom panels of Figure 4.4 illustrate the velocity field and depression of the optical surface for a single vertical vortex.

\subsubsection{Granule properties}

In this section, we analyse the statistical properties of granules as they appear in individual snapshots of the simulation runs, i.e. we do not consider the evolution or time-averaged properties of individual granules but their properties at a given point in time. From 33 snapshots for each simulation about 1000 to 2000 granules were detected (values see table 4.1). The time interval between two subsequent images considered was $\Delta t=300 \delta t$ where $\delta t$ is the simulation time step ( $\Delta t$ is thus on the order of 1 minute of stellar time). Since many granules live much longer than $\Delta t$, individual granules are likely to appear several times in this sample at different stages of their evolution.

For the F-, G-, and K-star simulations, the filling factor of granules as detected by our segmentation algorithm is between 67.5 and 69\% (including non-trackable granules). In the $\mathrm{M}$ stars, the granules have a slightly higher filling factor of about $72 \%$, which confirms the impression that the intergranular lanes of the $\mathrm{M}$ stars are narrower than the ones of hotter stars. These values are very close to the filling factor of 64 and $69 \%$ for the upflow area about one pressure scale height below the surface in all simulations (see Fig. 3.6). The asymmetry between up- and downflow area is mirrored in the asymmetry between granular and intergranular area, which we find are fairly robust and independent of the parameters used in our segmentation method.

Figure 4.5 shows histograms of the granule area. The size of the granules decreases for cooler, more compact stars (see Sect. 3.3.2). The spread of the size distributions of almost two orders of magnitude in area (one order of magnitude in granule diameter) is similar for all stars. For the Sun (G2V simulation), we find granule areas between 0.2 
Table 4.1: Detected granules.

\begin{tabular}{lrrrrrr}
\hline \hline Simulation & F3V & G2V & K0V & K5V & M0V & M2V \\
\hline \# of snapshots & 33 & 33 & 33 & 33 & 33 & 33 \\
time span [min] $_{\text {\# of granule snapshots }}^{\text {a }}$ & 28.2 & 24.8 & 34.9 & 28.4 & 28.4 & 24.8 \\
granule filling factor [\%] $^{\text {\# of granules }}{ }^{\text {b }}$ & 1788 & 1075 & 1684 & 1251 & 1598 & 1328 \\
${\text { \# of granules }\left(t_{\text {life }}>3 \text { min) }\right.}$ & $52.9 \pm 0.4$ & $68.1 \pm 0.8$ & $67.9 \pm 0.8$ & $69.0 \pm 0.5$ & $72.3 \pm 0.8$ & $72.6 \pm 0.9$ \\
\hline
\end{tabular}

a "granule snapshot" refers to a granule as it appears in a snapshot (no tracking/evolution)

b "granule" refers to granules tracked through a part of the considered image series 

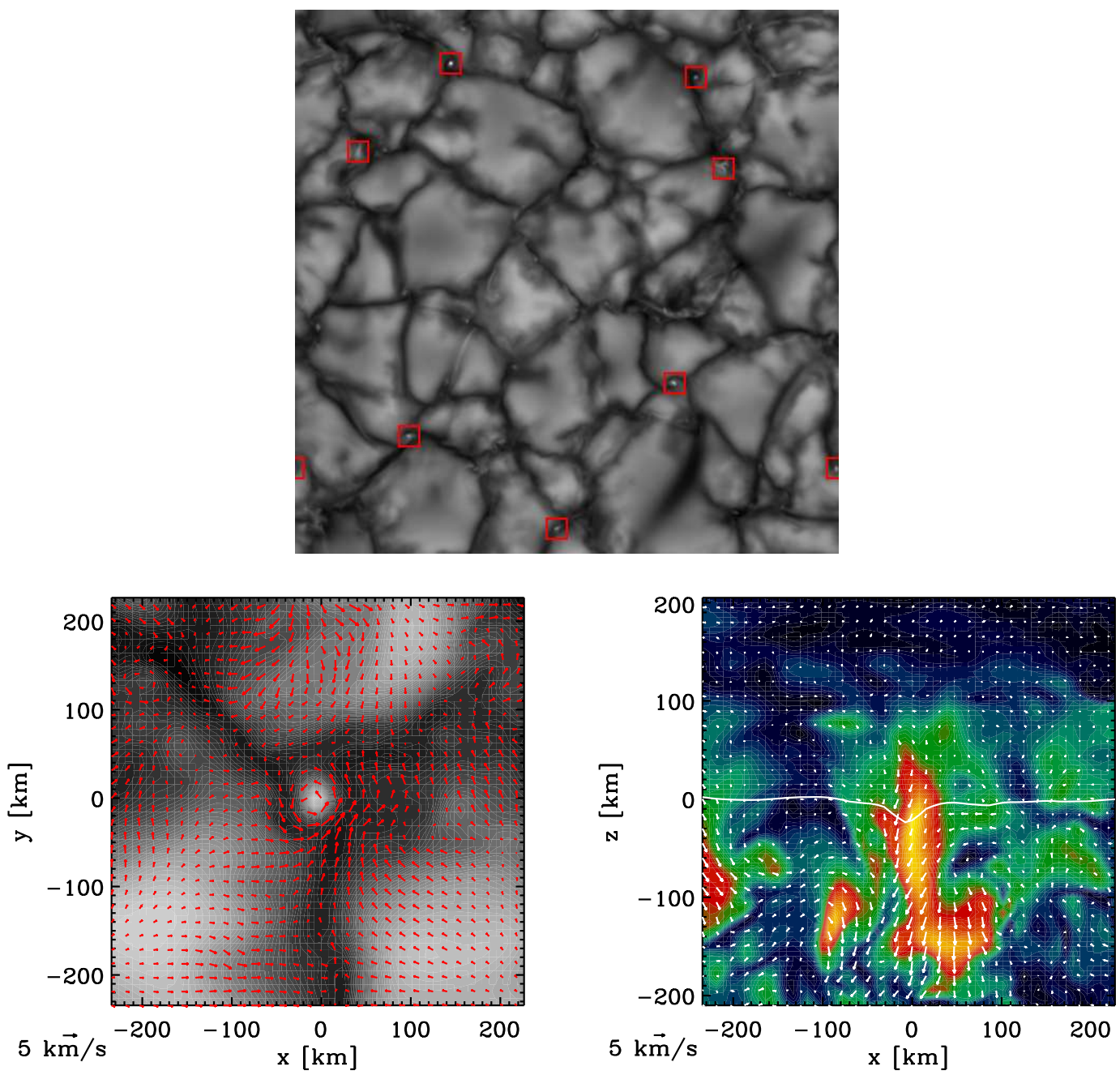

Figure 4.4: Vertical vortices. Top panel: intensity map with bright points $(\hat{I}>1.0)$ in a snapshot of the $\mathrm{K} 5 \mathrm{~V}$ simulation, all of which are associated with vertical vortices. Left bottom panel: zoom into the intensity map of a vertical vortex; the horizontal flow at $z=0$ is shown as red arrows. Right bottom panel: vertical cut through the vortex shown in the middle panel; the colour map shows the modulus of the vorticity $|\vec{\omega}|=|\nabla \times \vec{v}|$ (red/yellow indicating regions of high vorticity), the white arrows show the projection of the velocity onto the plane of the cut, the solid white line indicates the optical surface $\left(\tau_{\mathrm{R}}=1\right)$.

and $6 \mathrm{Mm}^{2}$, corresponding to diameters (assuming circular granule shape) of 0.5 to 2.5 $\mathrm{Mm}$ (0.6 to 3.5"). This is roughly consistent with the upper part of the size distributions in observations (e.g. Hirzberger et al. 1999). However, observationally obtained size distributions of solar granules show an increasing number of granules at decreasing size down to diameters of less than 0.5 ", while our numerically obtained distribution peaks at roughly 2". At least part of this discrepancy can be due to a different definition of a granule or to artifacts of the image reconstruction for the observations. A detailed comparison would require a degrading of our synthetic intensity maps and the segmentation of these "synthetic observations" and real observations of the same quality with the same 


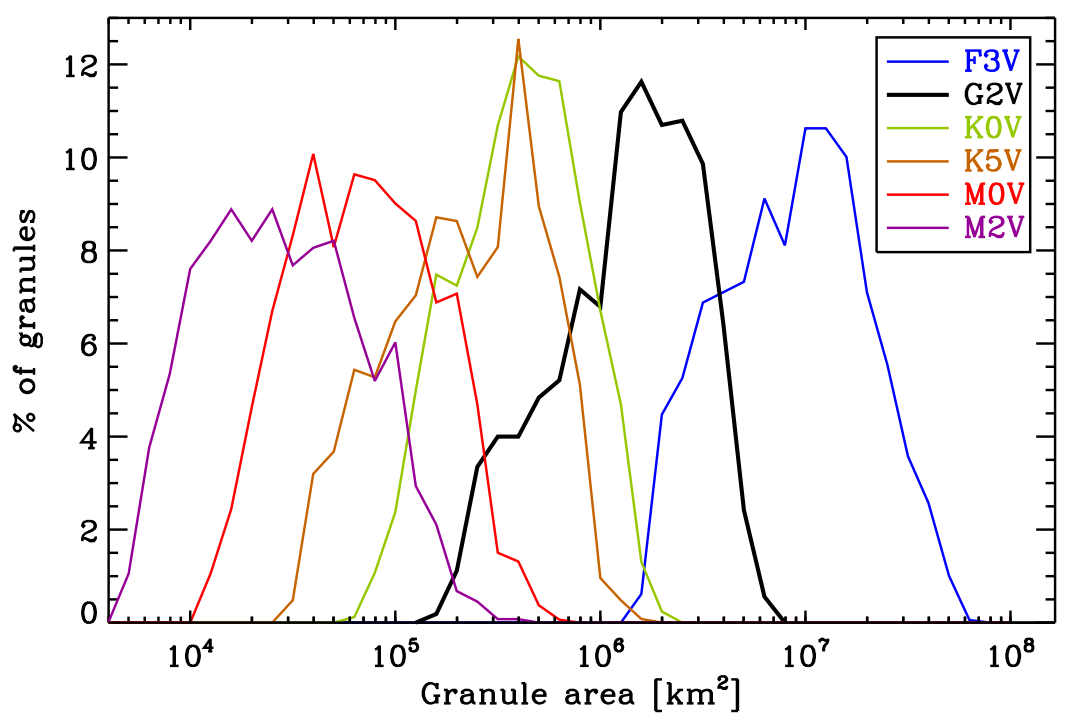

Figure 4.5: Histograms of granule area. The bins are logarithmically equidistant with a bin size of 0.1 dex.

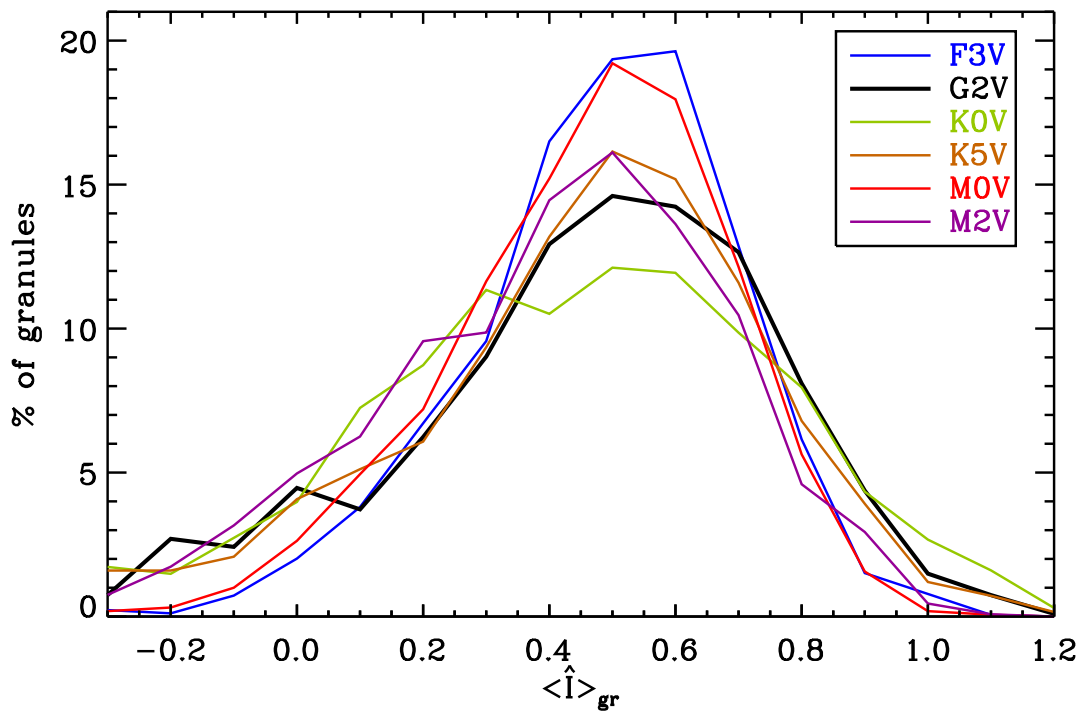

Figure 4.6: Histograms of granule brightness $\langle\hat{I}\rangle_{\mathrm{gr}}$, i. e. the normalised intensity fluctuation $\hat{I}$ averaged over single (snapshots of) granules (definition of $\hat{I}$, see Eq. (4.1)). The bins are linearly equidistant with a bin size of 0.1 .

algorithm. This is beyond the scope of this thesis.

Figure 4.6 shows histograms of the granule brightness. The quantity $\langle\hat{I}\rangle_{\mathrm{gr}}$ is the normalised intensity fluctuation $\hat{I}$ as defined in Eq. (4.1), averaged over the area of a granule. The histograms are similar for all spectral types, in spite of the different overall intensity 


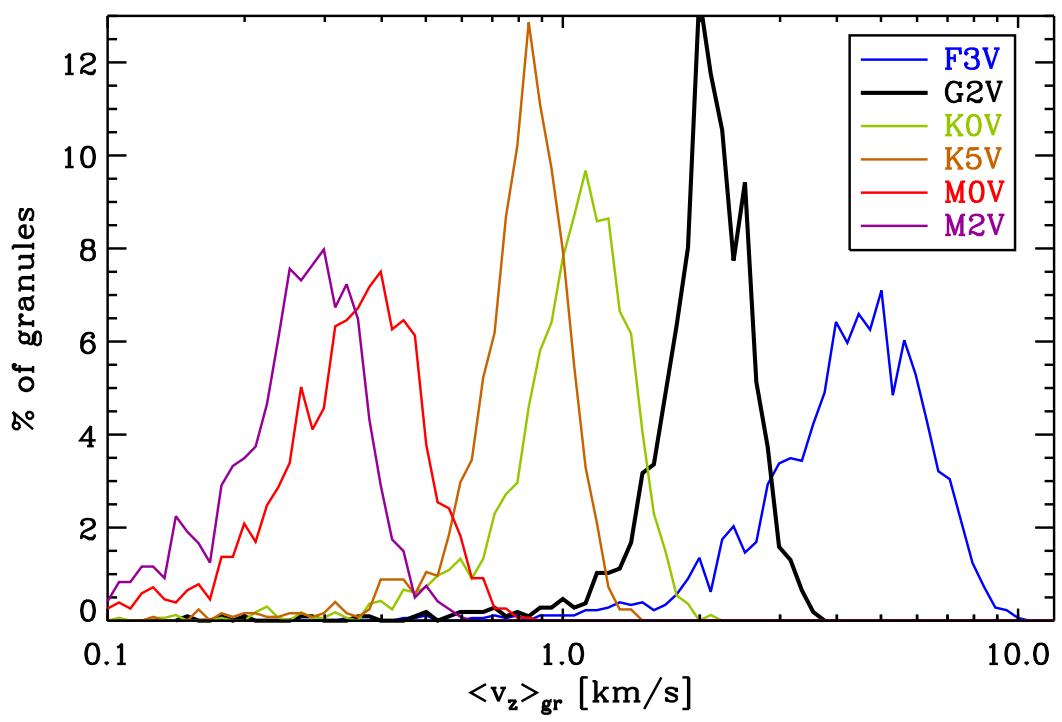

Figure 4.7: Histograms of $\left\langle v_{z}\right\rangle_{\mathrm{gg}}$, the vertical velocity averaged over the area of a (snapshot of a) granule. The bins are logarithmically equidistant with a bin size of 0.025 dex.

distribution (see Fig. 4.2). This can partly be attributed to the fact that the intensity fluctuation $\hat{I}$ is normalised by its standard deviation but also indicates the physical similarity of the convective upflow regions.

Figure 4.7 shows histograms of $\left\langle v_{z}\right\rangle_{\mathrm{gr}}$, which is the upflow velocity $v_{z}\left(\tau_{\mathrm{R}}=1\right)$ at the corrugated optical surface spatially averaged over the area of a granule. A very small fraction $(\lesssim 1 \%)$ of the detected granules have a negative mean vertical velocity; these can either be wrong detections or granules in a very late evolutionary state. In our logarithmic representation of the histograms only granules with an average upflow speed of more than $0.1 \mathrm{~km} \mathrm{~s}^{-1}$ are shown (more than $95 \%$ of the detected granules). The distributions show a marked peak at a velocity that can be regarded as the "typical convective velocity", $v_{\text {conv }}$. This peak shifts from about $4 \mathrm{~km} \mathrm{~s}^{-1}$ for the F3V simulation to about $0.3 \mathrm{~km} \mathrm{~s}^{-1}$ for M2V, and is roughly proportional to the rms value of $v_{z}$ at the optical surface (see Fig. 3.5).

We find a correlation of the brightness of a granule with its upflow velocity as well as with the amount of sub-structure in the granule. As a quantitative measure for the latter, we take the standard deviation $\sigma_{\mathrm{gr}}(\hat{I})$ of the normalised intensity fluctuation $\hat{I}$ within the granule area. Figure 4.8 shows the correlation between granule brightness, vertical velocity, and $\sigma_{\mathrm{gr}}(\hat{I})$ as scatter plots for the solar run $(\mathrm{G} 2 \mathrm{~V})$ and as binned scatter plots for all simulations. Brighter granules tend to have stronger mean upflow speeds and more sub-structure. The brightness of a granule is proportional to the convective energy flux directly below the optical surface, which in turn is roughly proportional to the vertical velocity. A correlation between mean velocity and brightness is therefore expected. The brighter granules also tend to have more brightness variation than the dimmer ones in all simulations. This is caused by a combination of many effects, such as inhomogeneities in the upflows, a stronger corrugation of the optical surface due to the higher average velocities in the brighter granules, more pronounced shock waves (in the F-star simulation), 

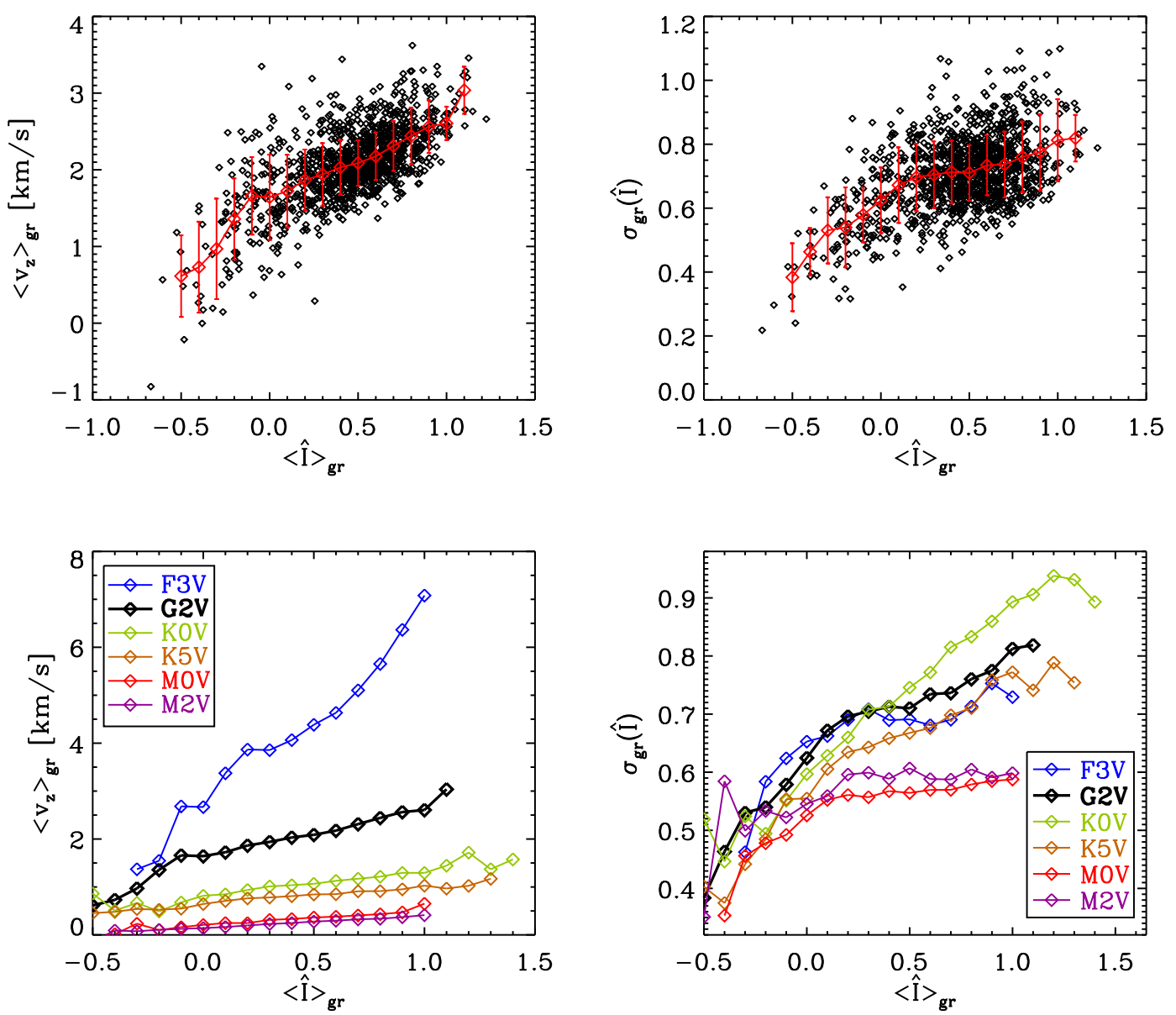

Figure 4.8: Correlations between granule brightness, $\langle\hat{I}\rangle_{\mathrm{gr}}$ and average vertical velocity (left panels) and standard deviation of intensity $\sigma_{\mathrm{gr}}(\hat{I})$ as measure for the sub-structure within the granules (right panels). The upper panels show scatter plots for the solar run $(\mathrm{G} 2 \mathrm{~V})$ where each black diamond represents a single granule. The red diamonds are binned averages (bin size 0.1 in $\hat{I}$ ) the error bars show the $1-\sigma$ scatter. In the lower panels the binned data are displayed for each of the six simulations.

and a less effective horizontal radiative diffusion owing to the shorter time span in which the convective elements rise through the near-surface layers where radiation becomes important.

Figure 4.9 shows the correlations of brightness and granule-averaged upflow velocity with the area of the granules. We find a correlation between area and mean brightness for the smaller granules, whereas for the larger ones the brightness saturates and correlation is lost. This is consisitent with solar observations (see, e.g,., Hirzberger et al. 1997). At least for large granules, there is negative correlation between size and mean vertical velocity (cf. Fig. 3.2). This might be due to buoyancy breaking: granules typically grow as more material wells up from below. Once their size exceeds a critical value of a few density scale heights the granule vanishes or splits (Nordlund et al. 2009). 

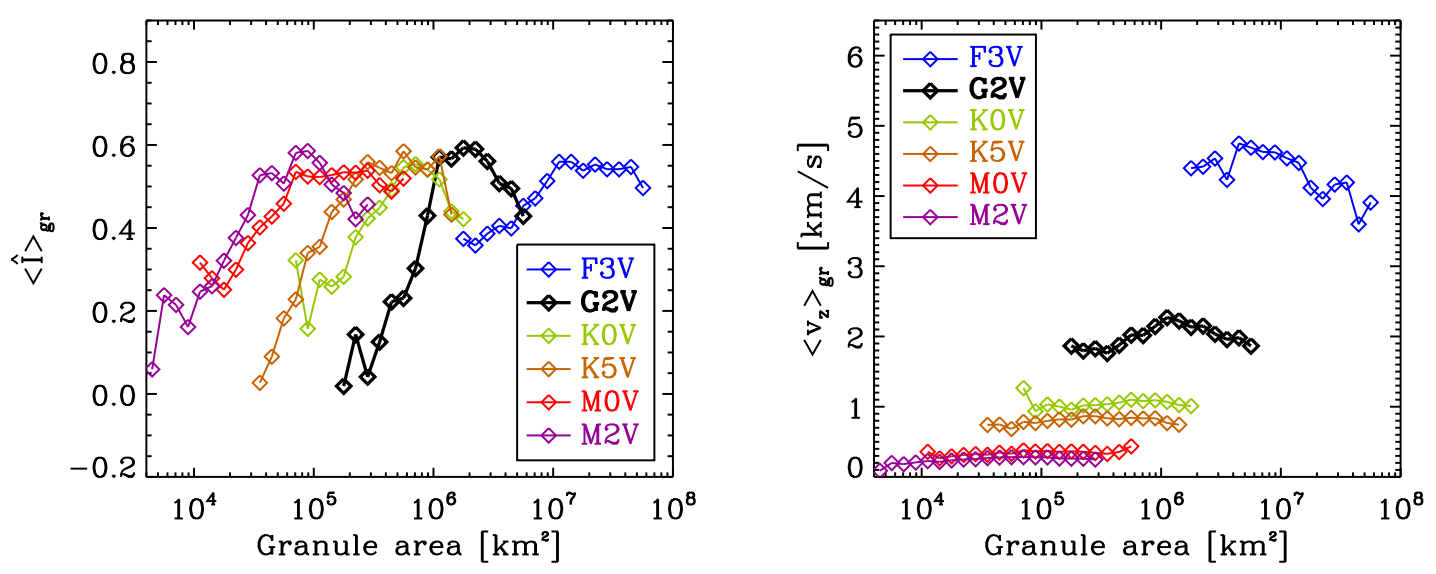

Figure 4.9: Correlations of granule area with granule brightness (left) and with vertical velocity (right) in binned scatter plots (cf. Fig. 4.8).

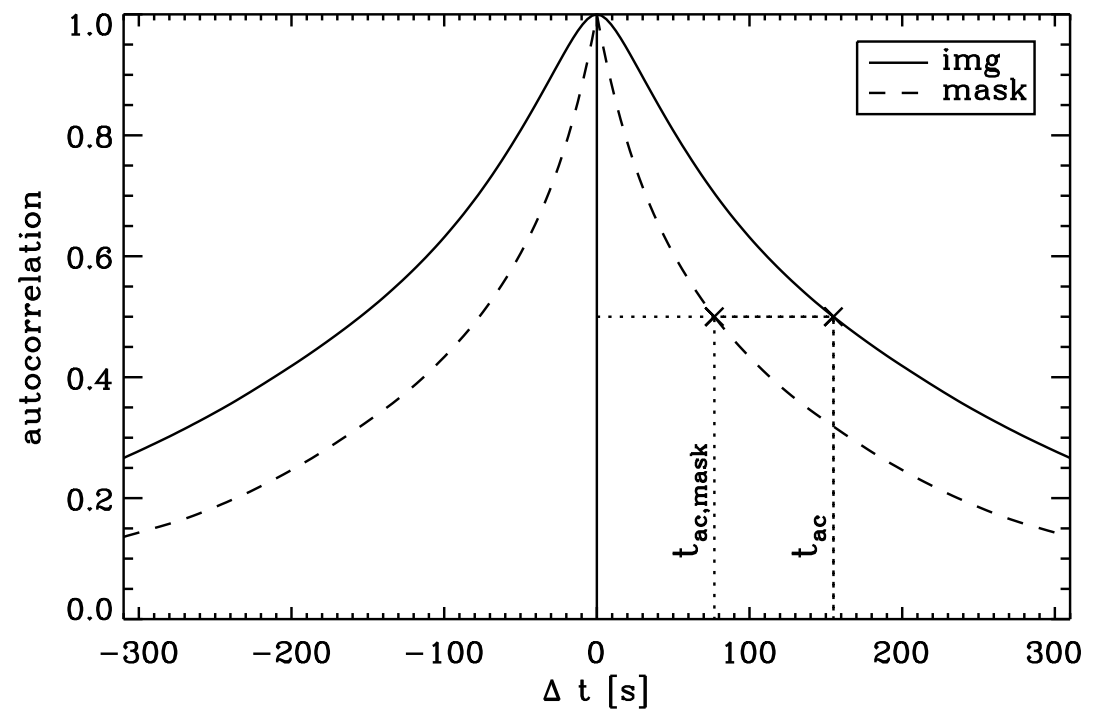

Figure 4.10: Autocorrelation function of the bolometric intensity images (img) and binary segmented masks (mask) as function of the time lag $\Delta t$ for the solar simulation $(\mathrm{G} 2 \mathrm{~V})$. The autocorrelation times $t_{\mathrm{ac}}$ and $t_{\mathrm{ac}, \text { mask }}$ (half width at half maximum) are marked.

\subsubsection{Granule lifetime}

In this section, we aim to determine the time-scale on which the granules evolve and the typical lifetime of granules. From solar observations, granule lifetimes of the order of several minutes have been reported (see, e. g., Title et al. 1989, Hirzberger et al. 1999).

One possible approach to define an evolution time-scale of the granulation pattern is autocorrelation (Title et al. 1989). We calculated the autocorrelation function for a time series of intensity images from our simulations as well as for a sequence of binary masks 
Table 4.2: Autocorrelation times and granule lifetimes in minutes

\begin{tabular}{lrrrr}
\hline \hline Simulation & $t_{\mathrm{ac}}$ & $t_{\mathrm{ac}, \text { mask }}$ & $\tau_{\text {life }}$ & $\tau_{\text {life,all }}$ \\
\hline F3V & 1.55 & 0.87 & 8.03 & 4.60 \\
G2V & 2.58 & 1.28 & 6.22 & 4.18 \\
K0V & 1.90 & 0.80 & 4.80 & 3.15 \\
K5V & 1.84 & 0.96 & 4.06 & 3.28 \\
M0V & 1.32 & 0.64 & 2.48 & 1.98 \\
M2V & 1.13 & 0.54 & 1.73 & 1.44 \\
\hline
\end{tabular}

of the segmented images (granules $=1$, intergranular lanes $=0$ ). We define the autocorrelation times $t_{\mathrm{ac}}$ and $t_{\mathrm{ac} \text {,mask }}$, respectively, as the time lag for which the autocorrelation has dropped by half. Figure 4.10 shows the autocorrelation function of the solar simulation $(\mathrm{G} 2 \mathrm{~V})$. The advantage of using the autocorrelation time is that it can be computed without previous image segmentation and does not depend on arbitrary definitions of granules. It does, however, not necessarily reflect the time-scale on which individual granules evolve: a pattern of randomly drifting, but otherwise unchanging granules would show a finite autocorrelation time although the individual granules show no evolution at all. The autocorrelation time therefore gives a pattern evolution time, which, in most cases, will be much shorter than the mean evolution time or the lifetime of individual granules. Table 4.2 lists the autocorrelation times $t_{\mathrm{ac}}$ and $t_{\mathrm{ac} \text {,mask }}$ for all simulations.

The lifetime of individual granules can be determined by tracking in time. For each simulation, about 250 to 600 granules were tracked with our algorithm (see Sect. 4.1 and Table 4.1). Our definition of the beginning and end of a "life" of a granule roughly follows Hirzberger et al. (1999): a granule starts its life either by fragmentation, merger, or apperance and dies by fragmentation, merger, or fading away. If a merger (or splitting) occurs which involves two very unequally large granules (with a critical area ratio of 15) only the smaller one ends (begins) its life, while the larger one survives. If both granules involved in a merger (splitting) are similar in size, both granules "die" (are "born"). Figure 4.11 shows histograms of the lifetime $t_{\text {life }}$ of the $\sim 250$ to 600 granules tracked with these criteria in each of the six time series. We only consider granules, whose lives lie entirely within the series. This implies that, for a time series starting at $t=0$ and ending at $t=T$, a granule with a lifetime of $t_{i}$ has to be born between $t=0$ and $t=T-t_{i}$ in order to be considered. The resulting histogram (shown as dotted line) therefore depends on the length $T$ of the time series. In order to remove this dependence, we corrected the histogram bins centred arround $t_{i}$ according to

$$
N_{i}=N_{i}^{*} \cdot \frac{T}{T-t_{i}}
$$

where $N_{i}^{*}$ is the number of detected granules within the lifetime bin $i$ (centred around $t_{i}$ ) and $N_{i}$ is the corrected number. These corrected histograms are shown as solid lines in Figure 4.11.

We find many small granules with a very short lifetime of less than 3 minutes, some of which are probably granule sub-structures or wrong detections. If one excludes these granules, the resulting histograms can be fitted quite well with an exponential function:

$$
N\left(t_{\text {life }}\right)=N_{0} \exp \left(-t_{\text {life }} / \tau_{\text {life }}\right) .
$$



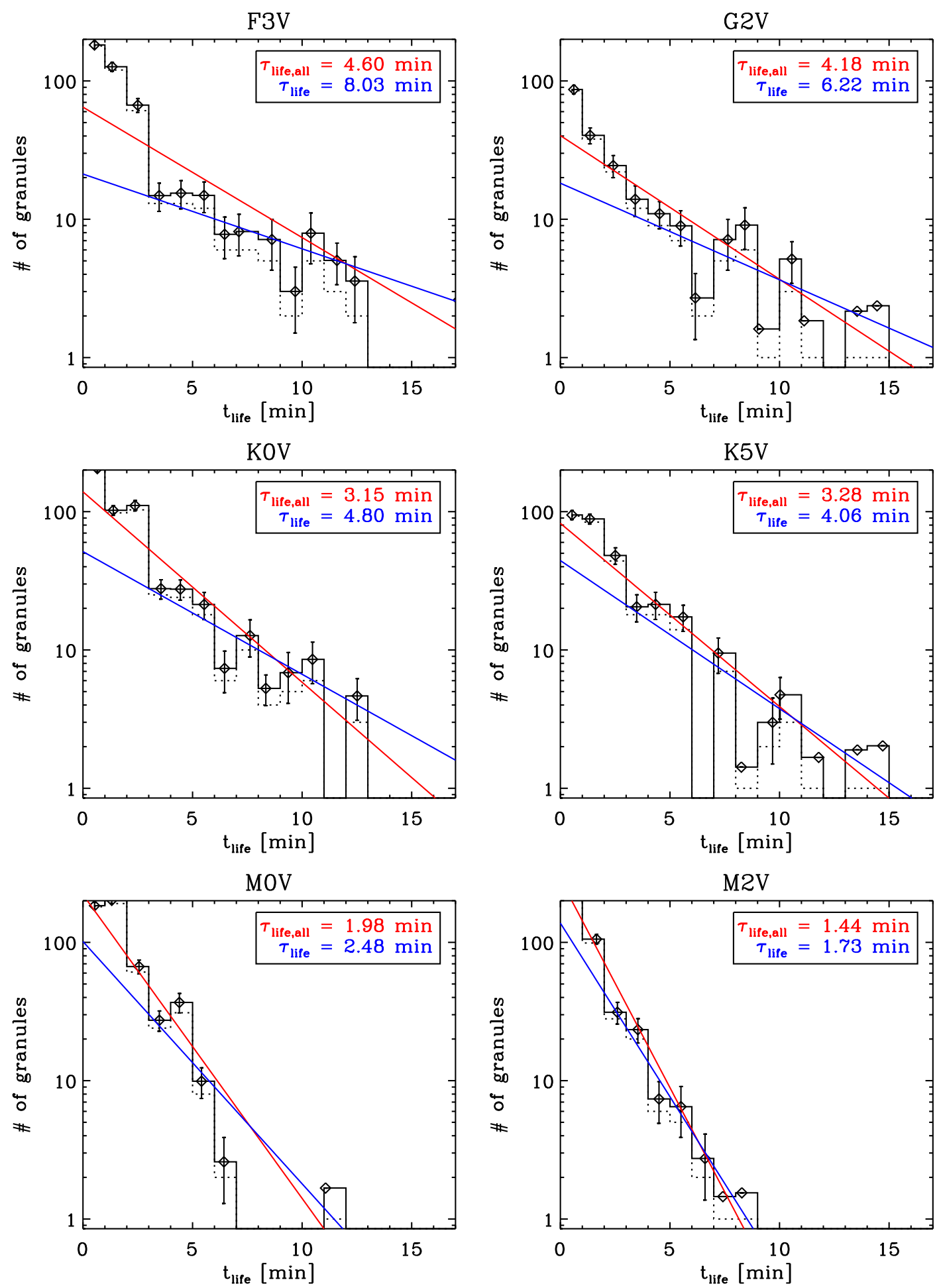

Figure 4.11: Lifetime histograms. The dotted black lines show the actual number of detected granules, $N_{i}^{*}$, while the solid black lines show the corrected granule number, $N_{i}$, of Eq. (4.3). The solid coloured lines are exponential least-square fits to all histogram bins (red), and to all but the first three bins (blue), respectively. 
Note that the short-lived granules are much more numerous (see table 4.1). For the sample from the solar simulation, we obtain $\tau_{\text {life }}=6.22 \mathrm{~min}$ considering the 48 granules with $t_{\text {life }} \geq 3$ min and $\tau_{\text {life,all }}=4.18$ min considering all 255 granules. This is consistent with the results by Title et al. (1989) and Hirzberger et al. (1999), who found exponential laws for the lifetime of observed solar granules with a time constant $\tau_{\text {life }}$ between 2.53 and 6.25 min (depending on the method of granule detection). Del Moro (2004) analysed three data sets of solar granulation and obtained values between 2.6 and 4.1 min for the long-lived granules. However, he argues that the distributions are better described by a stretched exponential or that the granule population should be divided into two parts with lifetimes of $t_{\text {life }}<2.5 \mathrm{~min}$ and $t_{\text {life }}>2.5 \mathrm{~min}$, respectively. This is also consistent with our histograms. In Table 4.2, we give the four different time-scales described in the previous paragraphs. There is an obvious trend towards shorter lifetimes for cooler stars. While the longer-lived granules of the F3V-star simulation live on average for 8 minutes, the M2V-star granules have a mean lifetime of less than 2 minutes. The autocorrelation time shows the same trend, except for F3V. This is probably due to the granule substructure and shock waves exhibited by this simulation, which both evolve on shorter time-scales than the granules.

Apart from the fact that many of the short-lived granules $\left(t_{\text {life }}<3 \mathrm{~min}\right)$ are very small, we do not find significant correlations between the granule lifetime and the temporal averages of the granule properties (area, intensity, mean vertical velocity). This, in the solar case, is in contrast to the results of Del Moro (2004), who found weak correlations between granule lifetime and mean area, mean intensity and maximum intensity of individual granules. However, our statistics (especially for granules with $t_{\text {life }}>5 \mathrm{~min}$ ) are relatively poor.

\subsection{Effects of the magnetic field on the granulation}

\subsubsection{Vertically emerging intensity}

As can be seen in Fig. 3.15 in Sect. 3.2, the magnetic simulations have a substantially different overall appearance of the intensity $I_{\text {bol }}(\mu=1)$. The granulation pattern is still visible in all magnetic simulations, but there are additional features. At low $B_{0}$, bright structures caused by magnetic field concentrations appear in the intergranular lanes. At higher values of $B_{0}$, the magnetic flux concentrations become more extended and some of them are dark. The threshold (in structure size and in $B_{0}$ ) between dominance of bright structures and dominance of dark structures appears to decrease towards the cooler stars in the model sequence. In the F3V and G2V simulations, the number and relative darkness of structures of reduced intensity is quite small (even at $B_{0}=500 \mathrm{G}$ ), while brightenings due to the magnetic field fill most of the intergranular lanes (especially in G2V) and can become very bright: the extended bright structure in the $500 \mathrm{G}$ snapshot of the F3V star in Fig. 3.15 at $(x, y)=(15 \mathrm{Mm}, 2.5 \mathrm{Mm})$ has a maximum intensity of $2.34\left\langle I_{\text {bol }}\right\rangle$, i. e. $\hat{I} \approx 7$, in terms of the normalised intensity fluctuation introduced in Eq. (4.1). In the $500 \mathrm{G}$ runs of the K-star simulations, the number density of dark structures is higher (in relation to the number density of granules) than in the hotter stars, and they become darker in terms of $\hat{I}$ (in their centres, typically $\hat{I}<-3$ ). The magnetic brightenings are also very prominent: 
the brightest structures in the K-star simulations often reach $I_{\mathrm{bol}}>2\left\langle I_{\mathrm{bol}}\right\rangle$ or $\hat{I}>10$; rare cases of up to $\hat{I}=20$ are observed in the K0V star simulation with $B_{0}=20 \mathrm{G}$ (see Fig. B.20 in Appendix B). The bright magnetic structures are brighter than the brightest vertical vortices observed in the non-magnetic K-star simulations. The reason for the extreme brightness of small magnetic structures in the K-star simulations is probably the same as for the vertical vortices (see Sect. 4.2.2): the low temperature sensitivity of the opacity between 4000 and $5000 \mathrm{~K}$ entails a strong effect of the gas pressure on $T\left(\tau_{\mathrm{R}}\right)$. Consequently, the reduction of the gas pressure shifts the $\tau_{\mathrm{R}}=1$ level in the flux concentrations to higher temperatures (relative to the effective temperature) in the $\mathrm{K}$ stars than in other stars. In the M-star simulations, brightenings due to the magnetic field are very small-scaled, less frequent, and less bright: while the brightest feature observed in the M0V-star simulations has still an intensity of $I_{\mathrm{bol}} \approx 1.6\left\langle I_{\mathrm{bol}}\right\rangle$ or $\hat{I} \approx 17$, the brightest structures in the M2V simulations do not exceed $I_{\mathrm{bol}} \approx 1.3\left\langle I_{\mathrm{bol}}\right\rangle$ or $\hat{I} \approx 13$. In the M stars, prominent dark structures evolve. Although these structures typically still have intensities around $0.85\left\langle I_{\mathrm{bol}}\right\rangle$ in $\mathrm{M} 2 \mathrm{~V}$, compared to the intensity contrast of the non-magnetic M2Vstar simulation of $\sigma_{I} \approx 0.02\left\langle I_{\mathrm{bol}}\right\rangle$, this is a considerable change. The filling factor of these dark regions is so high, that $\sigma_{I}$ is more than twice as large in the $500 \mathrm{G}$ run of the M2V simulation as in the non-magnetic run.

Figure 4.12 shows histograms of the vertical bolometric intensity normalised to the mean value of the respective non-magnetic simulation for the G2V and M2V simulations. For the solar (G2V) simulations, the peak of the distribution, which is at below-average intensity, increases somewhat in height from the non-magnetic run to $B_{0}=100 \mathrm{G}$, while the "shoulder" attributed to the granules shrinks. Both these effects are not directly related to the magnetic flux concentrations but an indirect consequence of the presence of the field: the area fraction of downflows increases as a result of the reduced downflow speed and the reduced density in the downflows. The logarithmic representation of the intensity histogram shown in the lower left panel of Fig. 4.12 indicates additional bright features with a low area fraction (the highest area fraction of very bright regions with $I_{\mathrm{bol}}>1.5\left\langle I_{\mathrm{bol}, 0 \mathrm{G}}\right\rangle$ is found in the $100 \mathrm{G}$ run). In the $500 \mathrm{G}$ run, dark regions appear in the $\mathrm{G} 2 \mathrm{~V}$ star, while the bright regions exhibit a lower intensity than in the $100 \mathrm{G}$ run (cf. Fig. 3.15). As dark magnetic regions (micropores) are usually surrounded by bright regions with a smooth transition, a substantial fraction of the magnetised area has a bolometric intensity which falls into the range of normal granulation. This explains the different overall shape of the 500 G-run histogram. For the M2V simulations, the dark component already appears in the $100 \mathrm{G}$ run. As the overall distribution is rather narrow, the dark magnetic features at about 80 to $90 \%$ of the mean intensity are prominent in the intensity histogram of the $500 \mathrm{G}$ run.

Figure 4.13 shows the average relation between the magnetic field modulus $B\left(\tau_{\mathrm{R}}=1\right)$ at the optical surface and the vertically emerging bolometric intensity (normalised to its mean value) for all three magnetic runs $\left(B_{0}=20 \mathrm{G}, 100 \mathrm{G}, 500 \mathrm{G}\right)$ of the F3V, G2V, K5V, and $\mathrm{M} 2 \mathrm{~V}$ simulations as binned scatter plots. The symbol sizes are scaled with the (logarithm of the) number of points per bin where this is lower than 1000 (i. e. $\sim 0.06 \%$ of a total of $1.57 \cdot 10^{6}$ points considered): very small symbols are thus statistically probably not significant. All curves show a systematic decrease from the first bin $(0-200 \mathrm{G})$ through the next few bins. This is a purely kinematic effect: the magnetic field accumulates in downflows, which are intrinsically darker than the granules as long as the field 

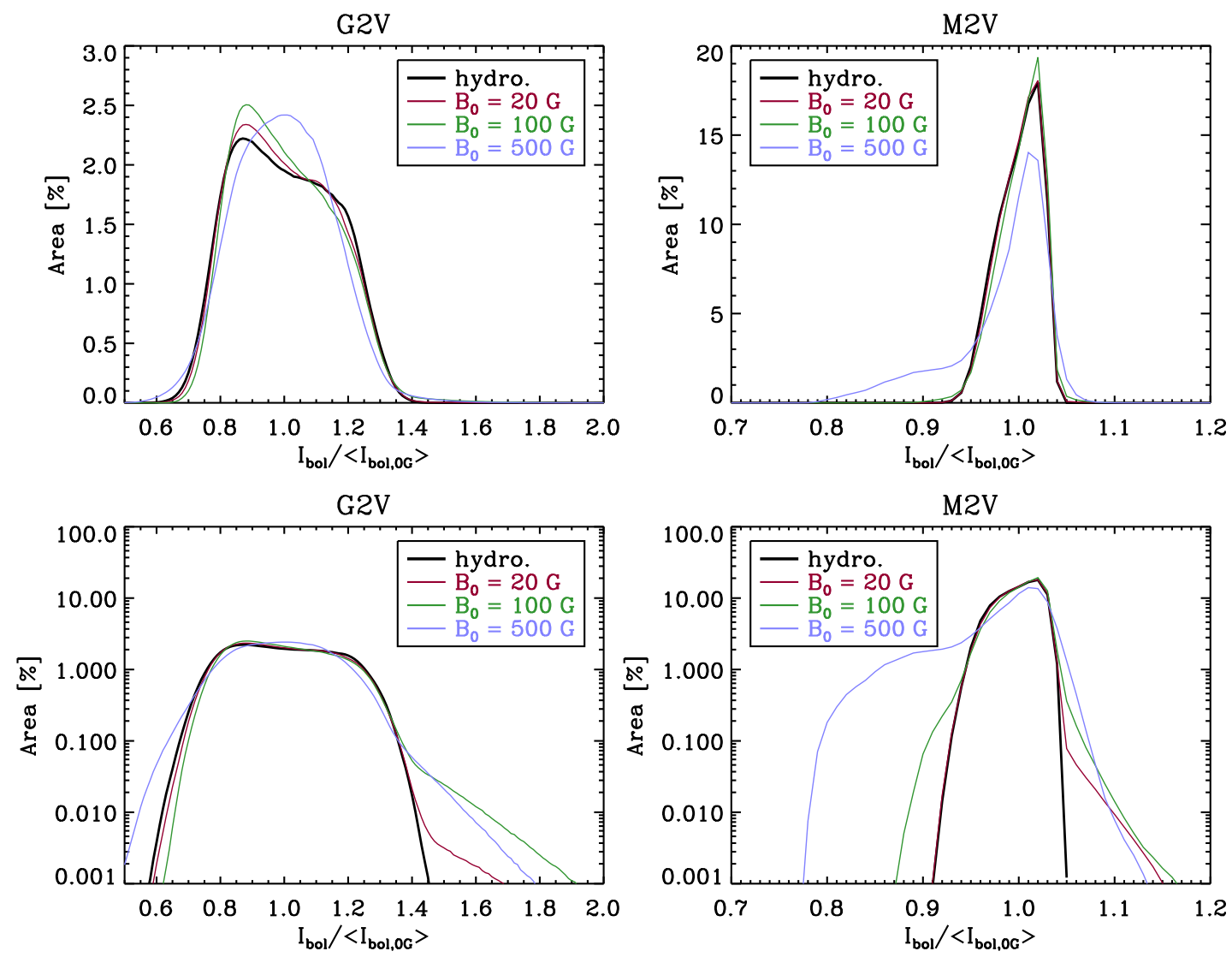

Figure 4.12: Intensity histograms of the G2V (left panels) and M2V (right panels) simulations. In both representations (upper panels: linear; lower panels: logarithmic), the histogram bins are linearly equidistant with a bin size of 0.01 .

strength is too small to influence the atmospheric structure and thus the local radiation field. From about $1 \mathrm{kG}$ on, there is a steep rise in most simulations, meaning that the local intensity is statistically higher where the local field strength at optical surface is higher. In all simulated stars shown, the slope of the $I-B$ relation becomes shallower (or changes sign) at larger values of $B_{0}$. This indicates the presence of larger structures, which have a dark centre or are entirely dark. The "error bars", which indicate the 1- $\sigma$ scatter within the bins, also become slightly larger at higher $B_{0}$, suggesting a bimodality of the intensity distribution in magnetic structures. The $\mathrm{M}$ stars (here, only the $\mathrm{M} 2 \mathrm{~V}$-star simulation is shown), qualitatively differ from the other simulations: Even at $B_{0}=20 \mathrm{G}$, the slope of the relation between $B\left(\tau_{\mathrm{R}}=1\right)$ and $I_{\mathrm{bol}}(\mu=1)$ is weaker than in the other stars (in relation to the scatter within the bins). At $B_{0}=100 \mathrm{G}$ and especially $B_{0}=500 \mathrm{G}$ the dark structures dominate. Only at very high field strength there remains a positive slope of the relation. As the symbol size suggests, this is, however, produced by a very small number of points (centres of small-scale bright regions).

As illustrated in Sect. 3.3, the different impact of the magnetic field on the vertical intensity is mainly due to the fact that the magnetic flux concentrations cause much shallower depressions in the M-stars than on other stars (cf. Figs. 3.27 and 3.26), which 

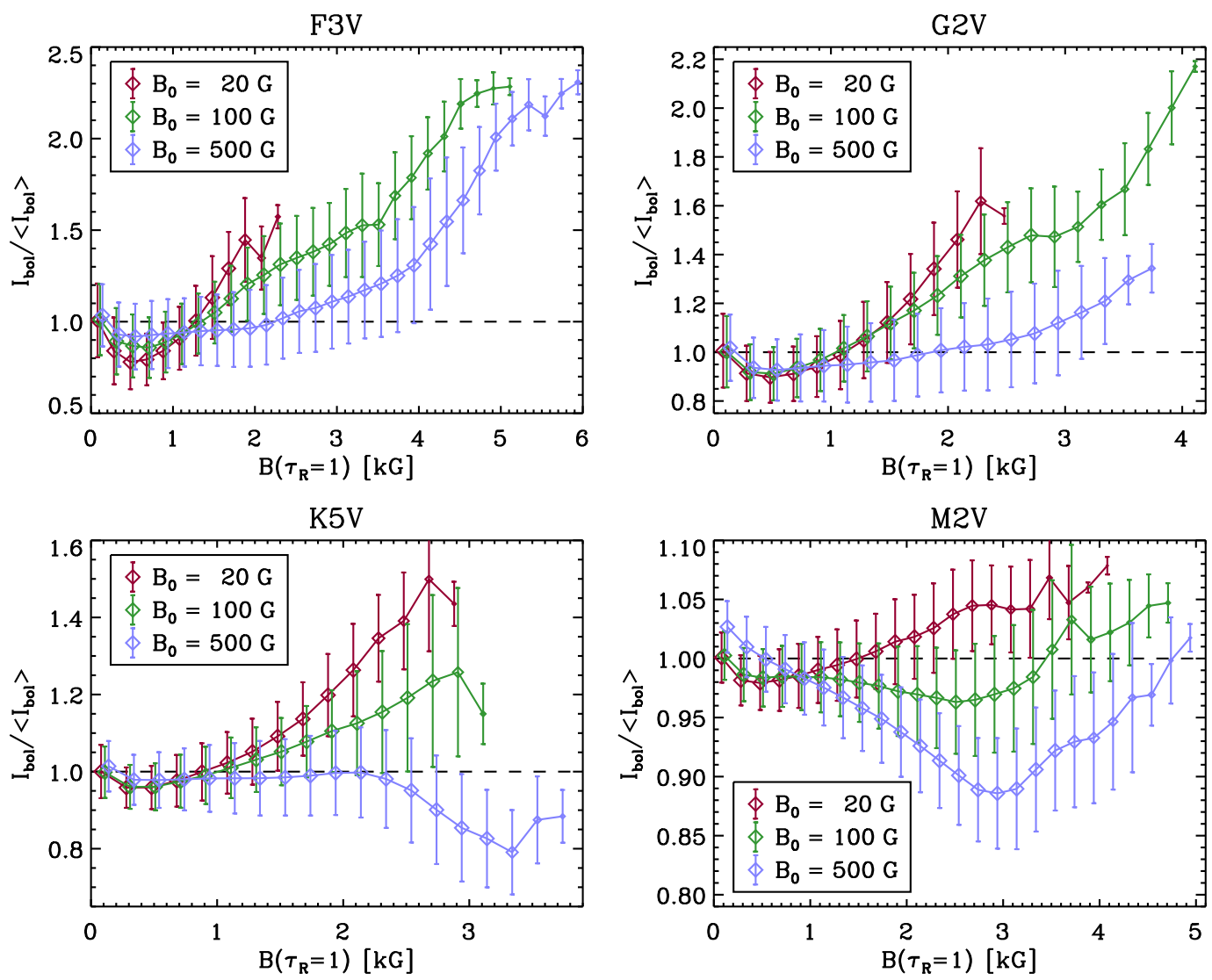

Figure 4.13: Relation between the magnetic field modulus $B\left(\tau_{\mathrm{R}}=1\right)$ at the optical surface and the vertical bolometric intensity $I_{\mathrm{bol}}(\mu=1)$ normalised with its mean value. The field is binned in steps of $200 \mathrm{G}$, error bars give the $1-\sigma$ scatter in the bins, the slight horizontal shifts between the plot symbols and error bars of different simulations are added for clarity. The symbol size is scaled with the logarithm of the number of points per bin where this number is below 1000 ( $\sim 0.06 \%$ of all points considered).

renders the side-wall heating inefficient even for structures much smaller than granules.

\subsubsection{Granule properties}

Although the magnetic flux is predominantly concentrated in the intergranular lane network, there are also some subtle effects of the magnetic field on the upflow regions, i.e. on the granules (cf. Figs. 3.29, 3.38, 3.42 and B.18). Therefore, one might expect that the average properties of the granules differ between non-magnetic and magnetic runs. For the F3V, G2V, and M2V simulations, the granulation segmentation described in Sect. 4.1 was carried out for the $100 \mathrm{G}$ runs. Here, the main differences in the statistical properties between the $100 \mathrm{G}$ run granulation and the non-magnetic granulation in these three stars are briefly summarised.

Figure 4.14 shows a comparison of the distributions of granule sizes and average vertical flow speed $v_{z}\left(\tau_{\mathrm{R}}=1\right)$ between the non-magnetic and magnetic runs. The differences are small: in the $100 \mathrm{G}$ runs of the F3V and G2V simulations, the mean gran- 

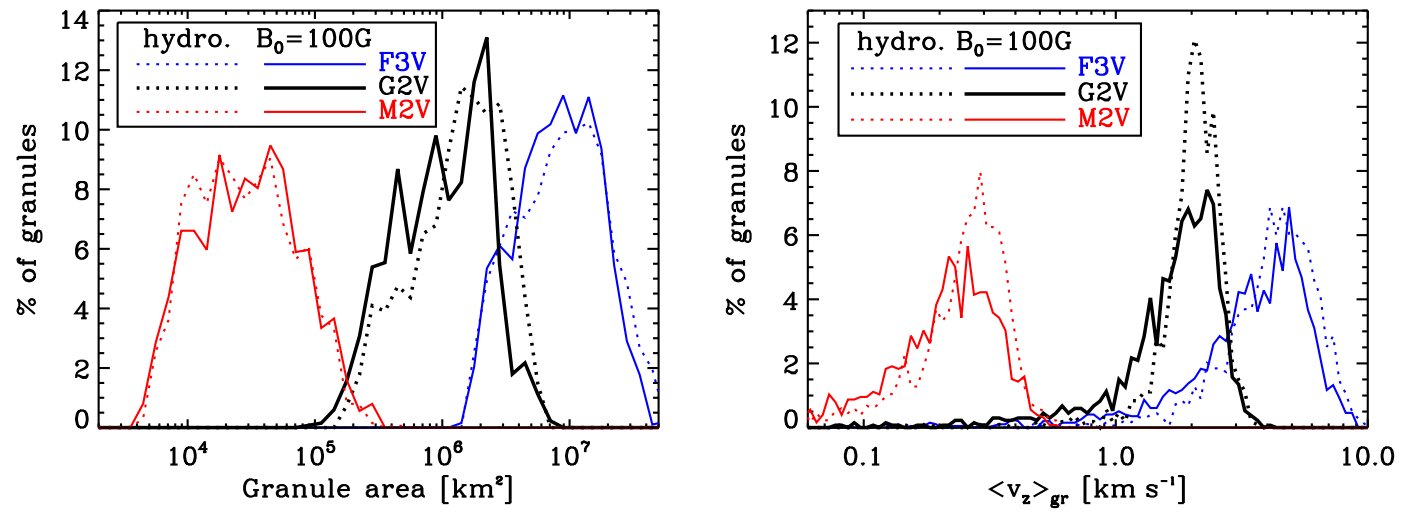

Figure 4.14: Histograms of the distribution of granule area (left) and granule averaged vertical speed at the optical surface (right). The dotted curves correspond to the nonmagnetic results also shown in Figs. 4.5 and 4.7. The bins are logarithmically equidistant with a bin size of 0.1 and 0.025 dex, respectively.

ule size is smaller by $12 \%$ and $23 \%$, respectively, than in the non-magnetic runs. The mean size of the granules in M2V simulation with $100 \mathrm{G}$ is slightly higher (7\%) than in the non-magnetic run. The vertical flow speed at the optical surface is reduced in all three magnetic runs compared to their non-magnetic counterparts: the mean values of the distributions are reduced by $18 \%(\mathrm{~F} 3 \mathrm{~V}), 20 \%(\mathrm{G} 2 \mathrm{~V})$, and $27 \%(\mathrm{M} 2 \mathrm{~V})$. This is in contrast to the upflow speed averaged on surfaces of constant optical depth (see Fig. B.21 in Appendix B), which shows a considerable decrease near the optical surface only for $B_{0}=500 \mathrm{G}$. The reason is the slightly higher area fraction of downflows in the granule areas detected by the algorithm. Morever, the averaging over the area of single granules gives more weight to small granules. As the effect of the magnetic field on the granules is mainly located at their edges (close to the downflows), smaller granules are more likely to show this influence in their average quantities than larger ones.

Figure 4.15 shows the relation between granule properties for the $100 \mathrm{G}$ and the non-magnetic runs. The left panel gives the relation between granule size and granuleaveraged normalised intensity fluctuation (granule brightness, hereafter). Small granules are brighter in the magnetic runs than in the non-magnetic runs, while large granules are less bright. This is probably an effect of the bright flux concentrations, especially when the brightening occurs not directly in the centre of a downflow lane but at the edge of a granule. In this case, the brightening is in some cases treated as part of the granule by the algorithm, which affects the mean brightness more strongly if the granule area is small. While this effect can be seen as a fault of the segmentation algorithm, the reduced intensity of large granules is probably a real effect.

The relation between granule brightness and upflow speed (right panel of Fig. 4.8 is only affected by the magnetic field for the less bright granules in the F- and G-star simulations, while for the M2V star, the smaller brighter granules seem to be affected more strongly than the less bright ones. For the F- and G-star simulations this again shows that small granules are more likely to show an influence of the magnetic field on their average properties than larger granules. In the M2V simulation, this can again be explained by the 

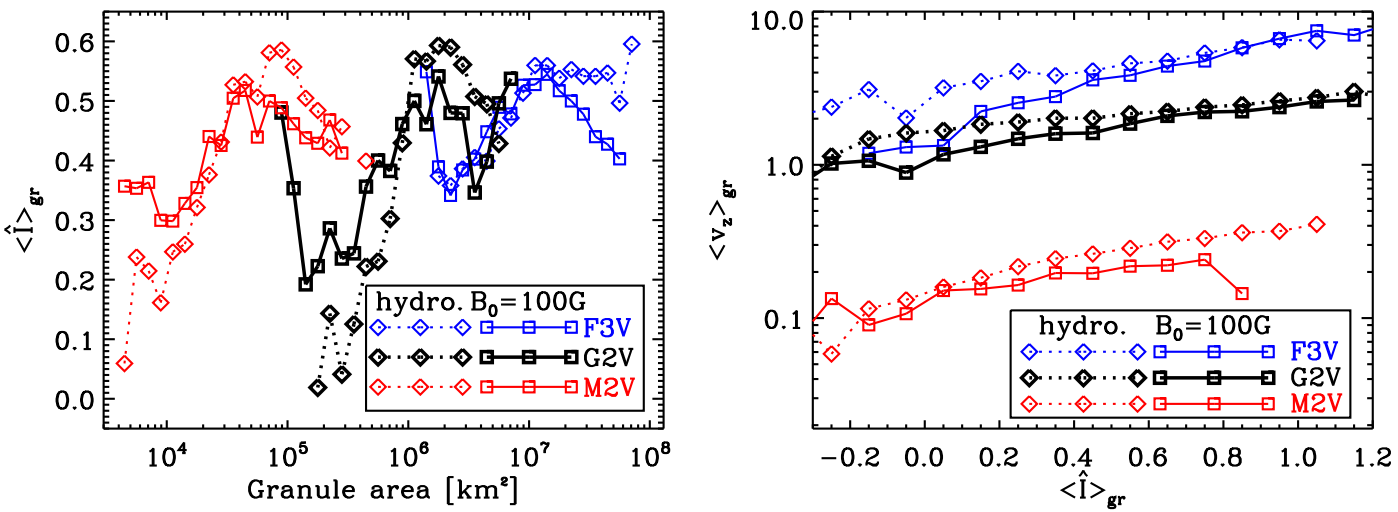

Figure 4.15: Relations between granule properties. Left: binned scatter plot of granule area and granule-averaged normalised intensity fluctuation $\langle\hat{I}\rangle_{\mathrm{gr}}$. Right: binned scatter plot of granule-averaged normalised intensity fluctuations $\langle\hat{I}\rangle_{\mathrm{gr}}$ and granule-averaged vertical speed at the optical surface. The diamonds and dotted lines represent the nonmagnetic simulations also shown in Fig.4.9 and Fig. 4.8.

magnetic brightenings: some small granules are brighter due to the magnetic brightenings at their edges (in the periphery of the micropores). As small granules tend to have lower upflow velocities in the M-type stars, the average vertical velocity of the granules goes down for bright granules.

\subsubsection{Temporal evolution of individual magnetic features}

Unfortunately, the magnetic bright structures caused problems with the stability of the segmentation algorithm, rendering a lifetime analysis for the granules as presented for the non-magnetic runs in Sect. 4.2.4 difficult for the magnetic runs. In this section, the temporal evolution of the magnetic features described in Sect. 4.3.1 is briefly discussed. In contrast to all other sections of this thesis, the results shown in this section are partly taken from earlier points in time than indicated in Fig. 2.2. This has presumably no significant impact on the qualitative results shown. For a more quantitative study, however, the magnetic simulations would have to be run considerably longer.

The bright magnetic elements (or "magnetic bright points", "magnetic BPs") change their appearance on a relatively short time scale of roughly one minute. They are moved around by the surrounding granules. Their lifetime is hard to define since they often merge or fragment. Especially in the $100 \mathrm{G}$ and $500 \mathrm{G}$ runs, they do usually not appear as isolated objects but rather as a network. The left column of Fig. 4.16 shows the evolution of some magnetic BPs in the K0V star simulation with $B_{0}=20 \mathrm{G}$. The time in each panel is given in minutes after the introduction of the field; the grey scale (only in this column of the figure) is saturated $2.5 \sigma_{I}$ below the mean intensity (black) and $7.5 \sigma_{I}$ above the mean intensity (white). The small feature around $(x, y)=(2.5 \mathrm{Mm}, 3.7 \mathrm{Mm})$, which is associated with a vortical downflow structure, changes shape, brightness, and position on a time scale of about one minute. The larger structure near $(x, y)=(1.7 \mathrm{Mm}, 2.8 \mathrm{Mm})$ is somewhat more stable. It is, however, involved in a series of mergers and fragmentations 

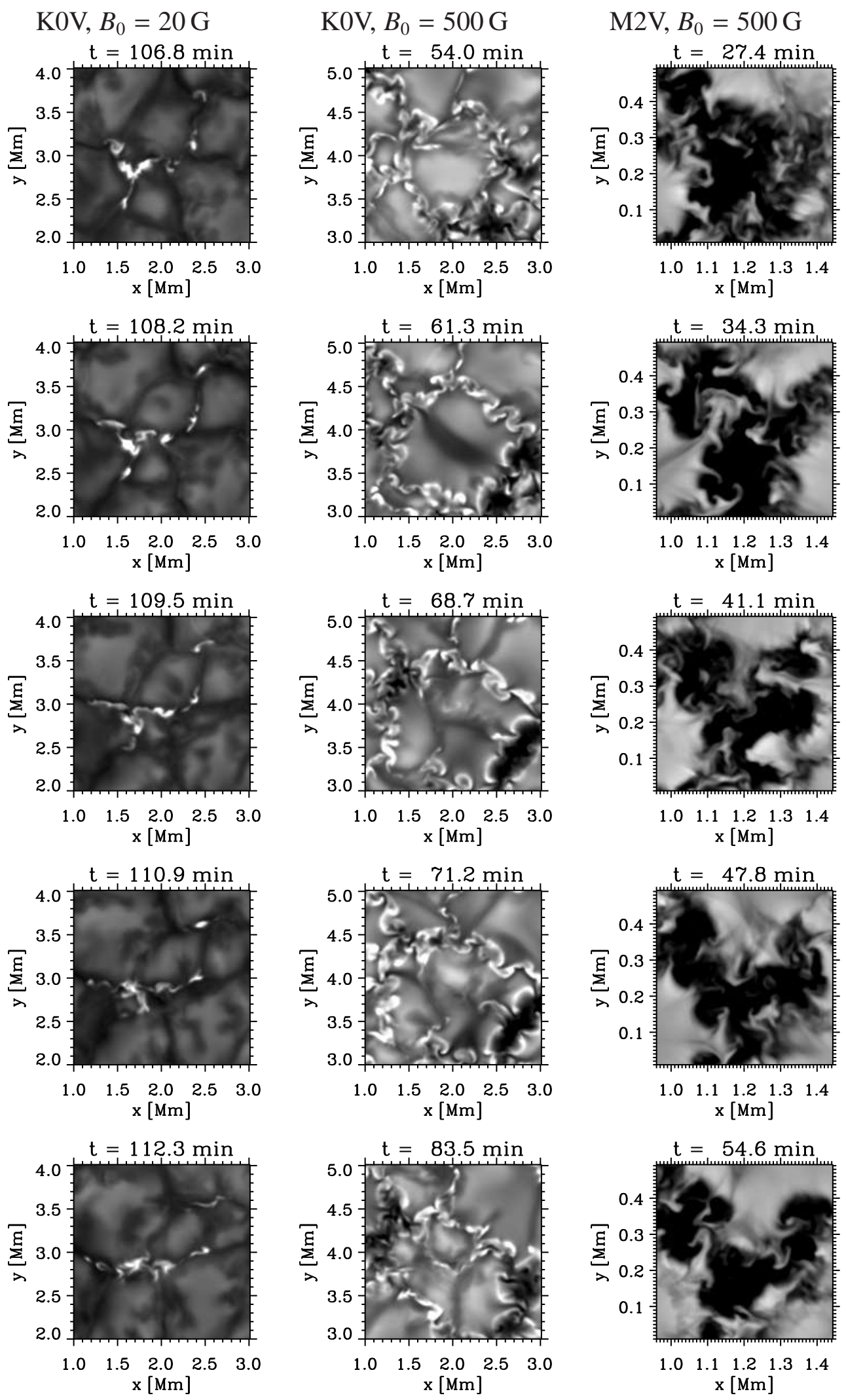

Figure 4.16: Temporal evolution of individual magnetic features. The grey scale saturates at $\hat{I}=-2.5$ (black) and $\hat{I}=7.5$ (left column) and 2.5 (middle and right column; white). 
on the same rapid time scale. The time scale and the merging and splitting behaviour are roughly consistent with observations of solar (chromospheric) magnetic BPs observed with Sunrise (Jafarzadeh et al. 2013).

As shown in Sect. 3.2 (cf. Figs. 3.15 and 3.16), the dark structures (or micropores) correspond to larger magnetic flux concentrations, which makes their evolution time scales longer than that of magnetic BPs. They are less moved around by the adjacent granules as the considerable amount of magnetic flux brakes horizontal flows. In the F-, G-, and K-star simulations, some of these structures can be followed through their entire evolution in our time sequence. They can "die" by fragmentation into smaller flux concentrations, which then become bright. However, as they usually appear at the vertices of mesogranules, they tend to reappear at similar locations after some time. This is visible for the micropores shown in the middle column of Fig. 4.16, which shows snapshots of the K0V simulation with $B_{0}=500 \mathrm{G}$. In the first image $(t=54 \mathrm{~min})$, there are two dark structures in the lower right corner of the region shown. During the next 15 min of stellar time, the two dark structures merge to one extended dark structure, while another small dark structure appears at the upper left corner of the region shown (second and third image). After a few more minutes, this smaller structure is gone, but at $t=83.5 \mathrm{~min}$ it has reappeared, while the larger structure has split into two again.

Compared to the micropores on hotter stars, the dark structures on M stars have an even longer lifetime. Not a single one of the larger of these structures dissolves during the simulation runs and fragmentation into smaller units is rare. The right column of Fig. 4.16 shows the evolution of the largest of these structures in the M2V simulation with $B_{0}=500 \mathrm{G}$ over roughly half an hour ( $\gtrsim 10$ granule lifetimes). Although some horizontal outflows from adjacent granules penetrate the structure and change the details of its shape, the general position and intensity of the structure is altered very little. As the simulations have not been run longer, it cannot be inferred if these dark regions will decay or fragment at some point. Their evolution time scale is, however, probably rather hours than minutes. 


\section{Limb darkening and centre-to-limb variation of the intensity contrast}

\subsection{Centre-to-limb variation of the intensity without mag- netic field ${ }^{1}$}

In this short section, we analyse the centre-to-limb variation of the intensity (limb darkening) and of the rms intensity contrast of the simulated stars. Analysis of the centre-to-limb variation of spectral lines follows in Sect. 6.2.2.

For the results presented in this section, we solved the radiative transfer using either only the continuum opacity or opacity distribution functions (ODFs) and directly integrated along single rays (with an adaptive increment to resolve the photospheric transition). For the opacities (continuum and ODFs), we used the same ATLAS9 data as for the radiative transfer of the hydrodynamical calculations (see Sect. 2.1.3). The narrow passbands in which the inclined view (Fig. 5.1) and the centre-to-limb variation for all simulations (Fig. 5.2) are presented, correspond to single wavelength bins of the ATLAS9 opacity data. For the limb darkening calculations in the Johnson filter bands (Fig. 5.3) several of these wavelength bins (B: 20, V: 25 , R: 35, I: 22) were combined with different weights according to the response functions of the filters (Johnson and Morgan 1951, Bessell 1990). Figure 5.1 shows intensity maps (continuum intensity at 400 to $410 \mathrm{~nm}$ ) of snapshots of the simulation for a line of sight inclined by $\theta=60^{\circ}$ (i. e. $\mu=0.5$ ) in the $y$-direction. The F- and G-type stars show bright granule edges facing the observer, which are viewed through the more transparent cool intergranular regions in front of them and hence appear brighter. These bright granule edges are less pronounced at this angle in the K-star simulations and absent in the M-star simulations. This can be attributed to the much smaller corrugation of the optical surface.

Figure 5.2 shows the centre-to-limb variation of the mean intensity $\langle I(\mu)\rangle /\langle I(\mu=1)\rangle$ and its normalised standard deviation $\sigma_{I}(\mu) /\langle I(\mu)\rangle$ (i. e. the rms intensity contrast) for four different continuum wavelengths. In all wavelength bands considered, the limb darkening is strongest in the two K-star simulations. Generally, limb darkening is stronger when the opacity decreases less steeply with height. ${ }^{2}$ In the lower photospheres of the K stars, the temperature is in the range of 4000 to $5000 \mathrm{~K}$, for which the opacity becomes almost independent of temperature, while in the photospheres of most other stars the opacity increases rapidly with temperature. Consequently, the opacity drops less steeply with

\footnotetext{
${ }^{1}$ This section has been published as Sect. 5 of Beeck et al. (2013b).

${ }^{2}$ more precisely: when the absorption coefficient $\kappa \varrho$ decreases less steeply with decreasing temperature.
} 

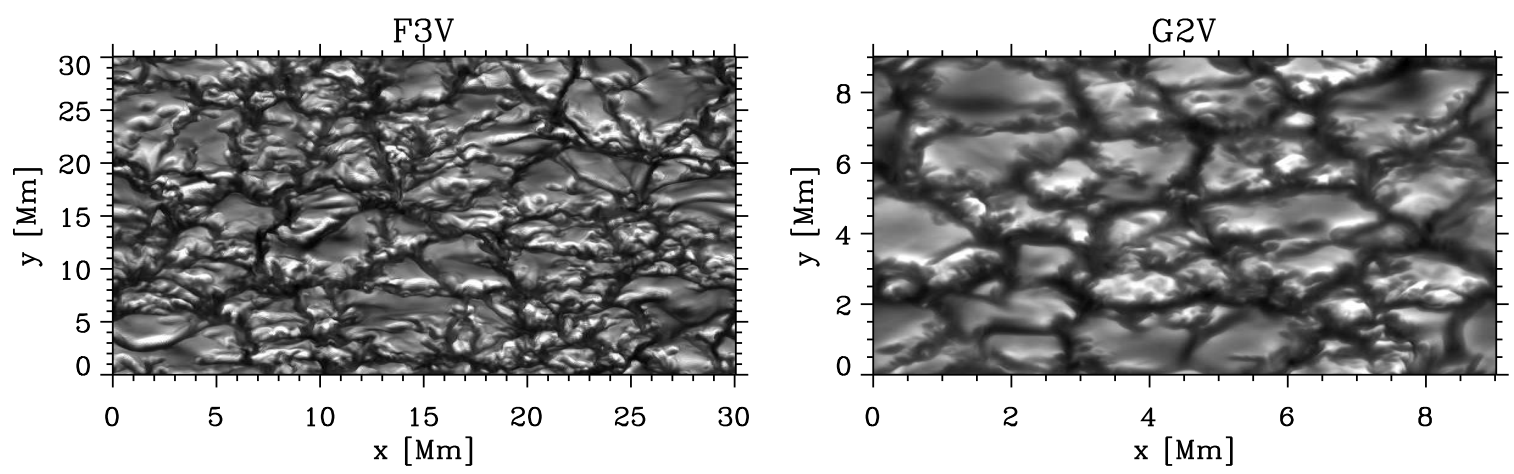

$\mathrm{KOV}$
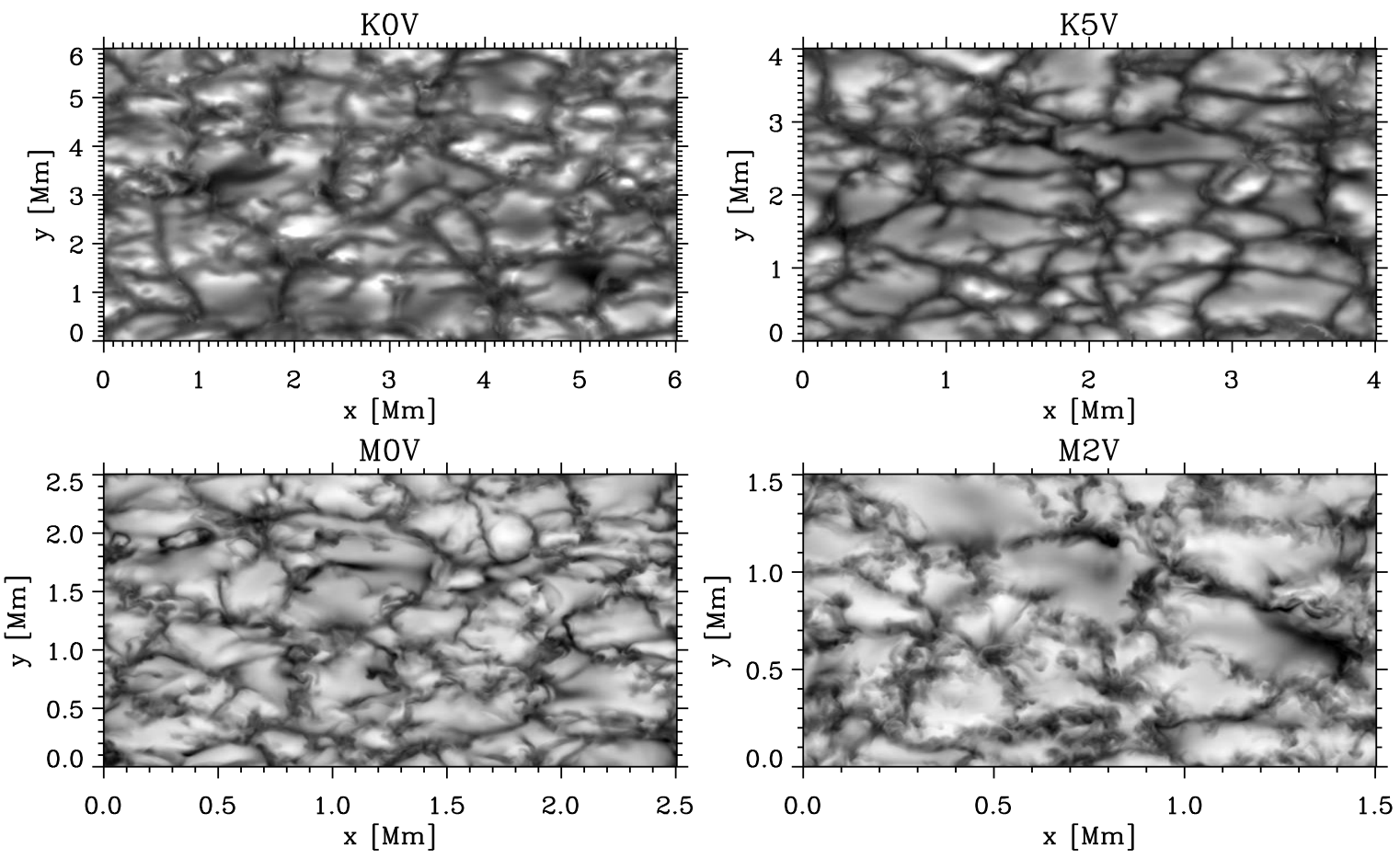

Figure 5.1: Maps of the intensity at $\mu=0.5$ for a wavelength band in the blue (400$410 \mathrm{~nm})$. The grey scale is saturated at \pm 3 standard deviations.

height in the $\mathrm{K}$ stars as compared to the other types, resulting in a stronger limb darkening.

As the source function (here assumed to be Planckian) depends more strongly on temperature towards shorter wavelengths, the limb darkening is strongest in the blue wavelength band $(400-410 \mathrm{~nm})$. Our most extreme case is our K5V star, for which the blue band intensity at $\mu=0.1$ is less than $15 \%$ of the disc-centre value.

While the limb darkening is qualitatively similar for all stars, the centre-to-limb variation of the intensity contrast changes qualitatively with effective temperature. For most stars, the intensity contrast decreases towards the limb, as the optical surface moves upwards where temperature fluctuations are smaller. However, for the F3V star, the contrast increases with decreasing $\mu$ (except very near to the limb). The reason for this behaviour is the strong corrugation of the optical surface: the "naked" granules are separated by deep, optically thin trenches, through which radiation can escape at an inclined angle (see also Fig. 5.1). As the granule side walls have less efficient radiative cooling than the gran- 

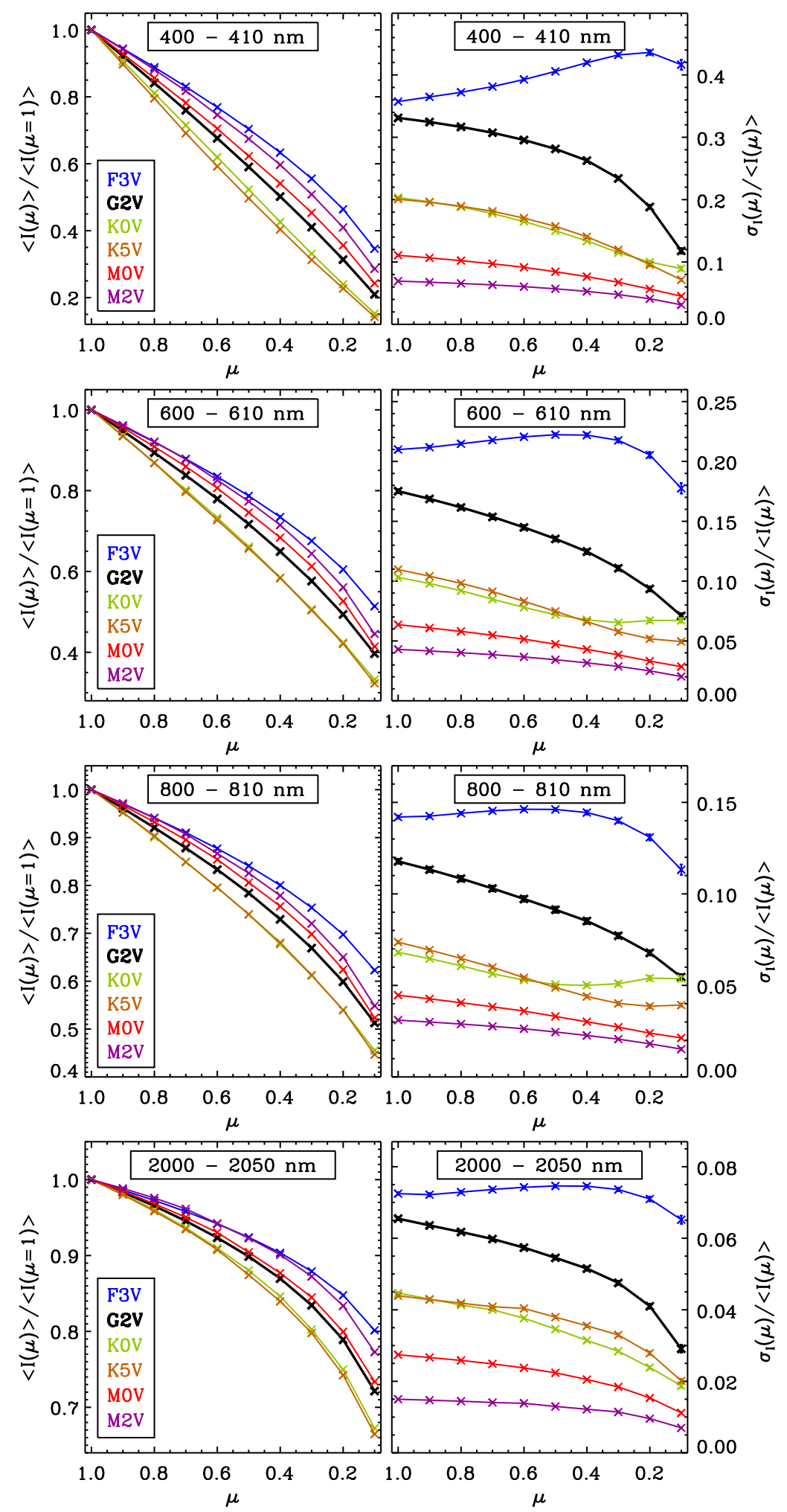

Figure 5.2: Centre-to-limb variation of the mean intensity $\langle I\rangle$ (left panels) and the rms intensity contrast $\sigma_{I} /\langle I\rangle$ (right panels) in four different wavelength bands centred at 405 , 605,805 , and $2025 \mathrm{~nm}$, respectively. Note the different $y$-scale for each panel. 
ule tops, the temperature contrast between the granule tops and the hot side walls is very high and so, as $\mu$ is decreased, the intensity contrast increases as more bright side walls come into view. At $\mu \lesssim 0.3$ this effect is over-compensated by the normal centre-to-limb decrease of contrast due to the increasing geometrical height of the optical surface. As the corrugation of the optical surface is less pronounced in the cooler stars, the intensity contrast decreases monotonically with decreasing $\mu$ in the $\mathrm{G}$ and $\mathrm{M}$ stars; the $\mathrm{K}$ stars (particularly the K0V star) show, however, an almost constant or even slightly increasing intensity contrast at low $\mu$ at some wavelengths. This is mainly produced by very bright regions behind intergranular lanes. As explained in Sect. 4.2.2, the temperatureinsensitive regime of the opacity between 4000 and $5000 \mathrm{~K}$ is responsible for a higher sensitivity of $T(\tau=1)$ to density fluctuations. For some extended intergranular lanes with particularly low density this leads to a significant brightening of the granule edges behind them at strongly inclined view. This effect might also be responsible for the fact that the two K-star simulations have similar contrasts, while it is generally decreasing with decreasing effective temperature: in the $\mathrm{K} 5 \mathrm{~V}$ simulation, the temperature-insensitive opacity regime is reached right at the optical surface in the granules (rather than in the intergranular lanes). Consequently, small density perturbations enhance the rms contrast of the granule intensities and thus the overall intensity contrast of the K5V simulation.

Figure 5.3 shows a comparison of the limb darkening of the K0V simulation with that of an ATLAS model with $T_{\text {eff }}=4750 \mathrm{~K}, \log g[\mathrm{cgs}]=4.5$, turbulent velocity $v_{\text {turb }}=$ $1 \mathrm{~km} \mathrm{~s}^{-1}$, and solar metallicity by Claret (2000). The typical relative difference between our 3D calculation and the 1D model is mostly below $2 \%$. The disc-integrated effect of these deviations on the total stellar radiance is between 0.4 and $0.5 \%$ in all bands. Carrying out the same comparison between our solar model (G2V) and the corresponding 1D results by Claret (2000), we find relative differences of up to about $8 \%$ (B band) and a disc-integrated effect between $0.2 \%$ (I band) and $1.6 \%$ (B band). We also compared our results to observational data of solar limb darkening by Neckel and Labs (1994) for a limited number of continuum wavelengths between 400 and $900 \mathrm{~nm}$ and found deviations mostly below $5 \%$. The statistical uncertainties of our limb darkening results are very small: the relative error of $\langle I(\mu)\rangle$ is of the order of $10^{-3}$, estimated from the scatter between the six snapshots. However, we expect somewhat larger systematic errors due to the opacity binning in our simulations with only four bins (Beeck et al. 2012).

\subsection{Effect of the magnetic field}

As the magnetic field has an impact on the corrugation of the optical surface (see Sect. 3.3.1), the centre-to-limb variation of the radiation leaving the star is affected by the presence of a magnetic field. Figure 5.4 shows the continuum intensity in a narrow passband between 400 and $410 \mathrm{~nm}$ of the six simulations with $B_{0}=500 \mathrm{G}$, viewed at an inclination of $60^{\circ}$ (i. e. $\mu=0.5$ ). In comparison to the non-magnetic simulations (see Fig. 5.1) there are some clear differences visible in the images. In the F-star simulation, the magnetic flux concentrations cause such deep depressions in the optical surface, that the intergranular lanes which contain magnetic flux vanish from sight behind the granules in front of them. The strong evacuation in the flux concentration above the optical surface, however, renders them more transparent for the radiation from the granules behind. Consequently, 


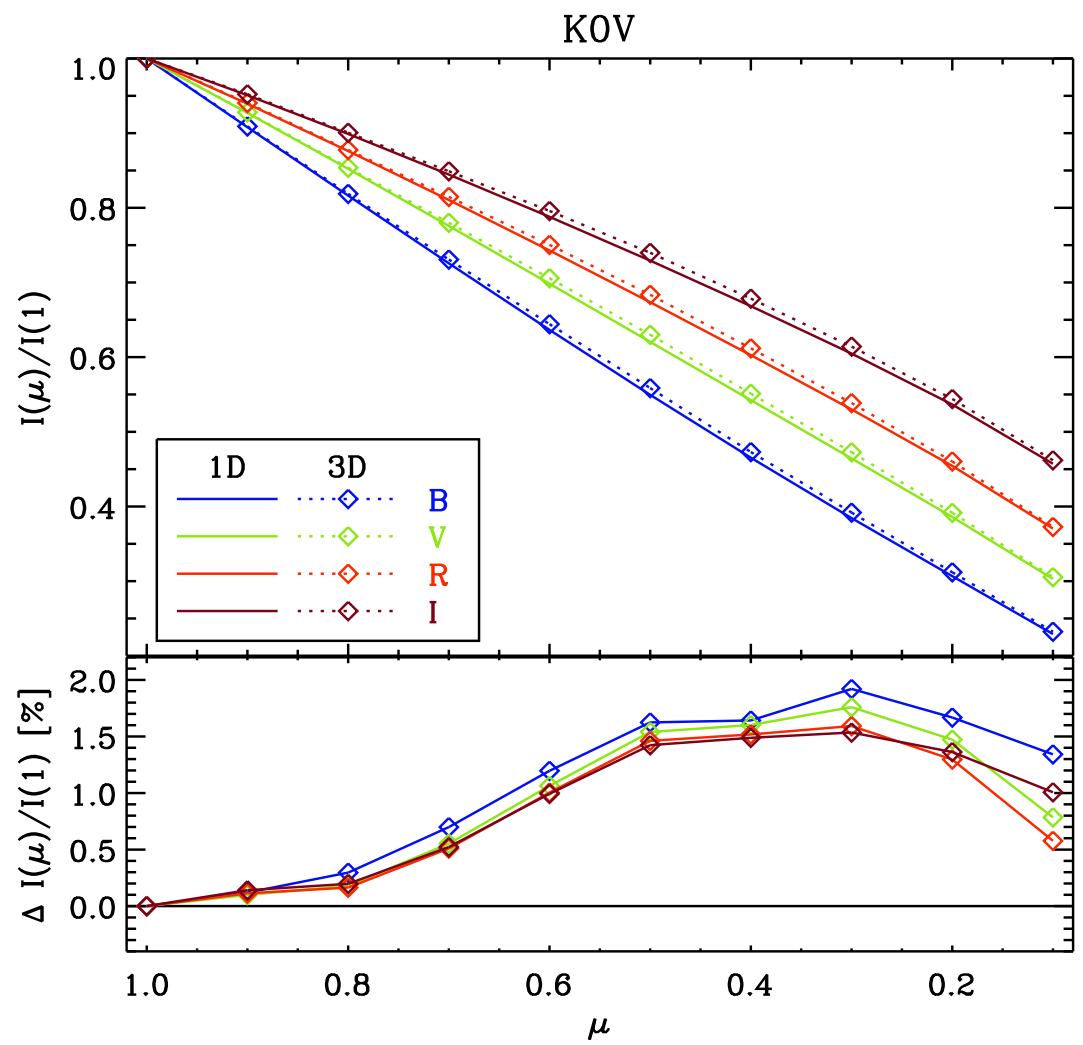

Figure 5.3: Limb darkening. Comparison of the limb darkening between the K0V MURaM simulation (diamonds and dashed curves) and a 1D ATLAS model (solid curves) with similar parameters $\left(T_{\text {eff }}=4750 \mathrm{~K}, \log g[\mathrm{cgs}]=4.5, v_{\text {turb }}=1 \mathrm{~km} \mathrm{~s}^{-1}\right.$, solar metallicity) by Claret (2000). The four passband filters are the Johnson B, V, R, and I passbands (Johnson and Morgan 1951, Bessell 1990). The lower subplot shows the relative differences betwen $1 \mathrm{D}$ and $3 \mathrm{D}$ results.

granules seen through flux concentrations appear brightened. To a lesser extend, this is also visible in the G2V simulation and is consistent with solar observations of "faculae" as well as MHD simulations (see, e. g. Carlsson et al. 2004, Keller et al. 2004, Steiner 2005). The thin dark contours seen especially in front of bright granules are produced by the cooler gas directly above the granules where the optical path through this gas is particularly long ("limb darkening" of single granules). In the G- and K-star simulations, the brightenings are more confined to the side walls of the granules behind flux concentrations. A few micropores are visible, but less prominent than at $\mu=1$ (cf. Fig. 3.15). In contrast, the M-star simulations still show prominent dark regions at this angle. However, compared to the vertical view, the magnetic regions are associated with more bright regions (side walls of the depressions).

Figure 5.5 shows the centre-to-limb variation of the continuum intensity in the $500 \mathrm{G}$ runs and non-magnetic runs of the $\mathrm{G} 2 \mathrm{~V}$ - and $\mathrm{M} 2 \mathrm{~V}$-star simulations in four narrow passband filters (for analogous plots for the other four stars see Fig. B.22 in Appendix B). The presence of the magnetic field reduces the limb darkening in all wavelength bands considered. In the case of the G- and K-star simulations, this weakening of the limb dark- 

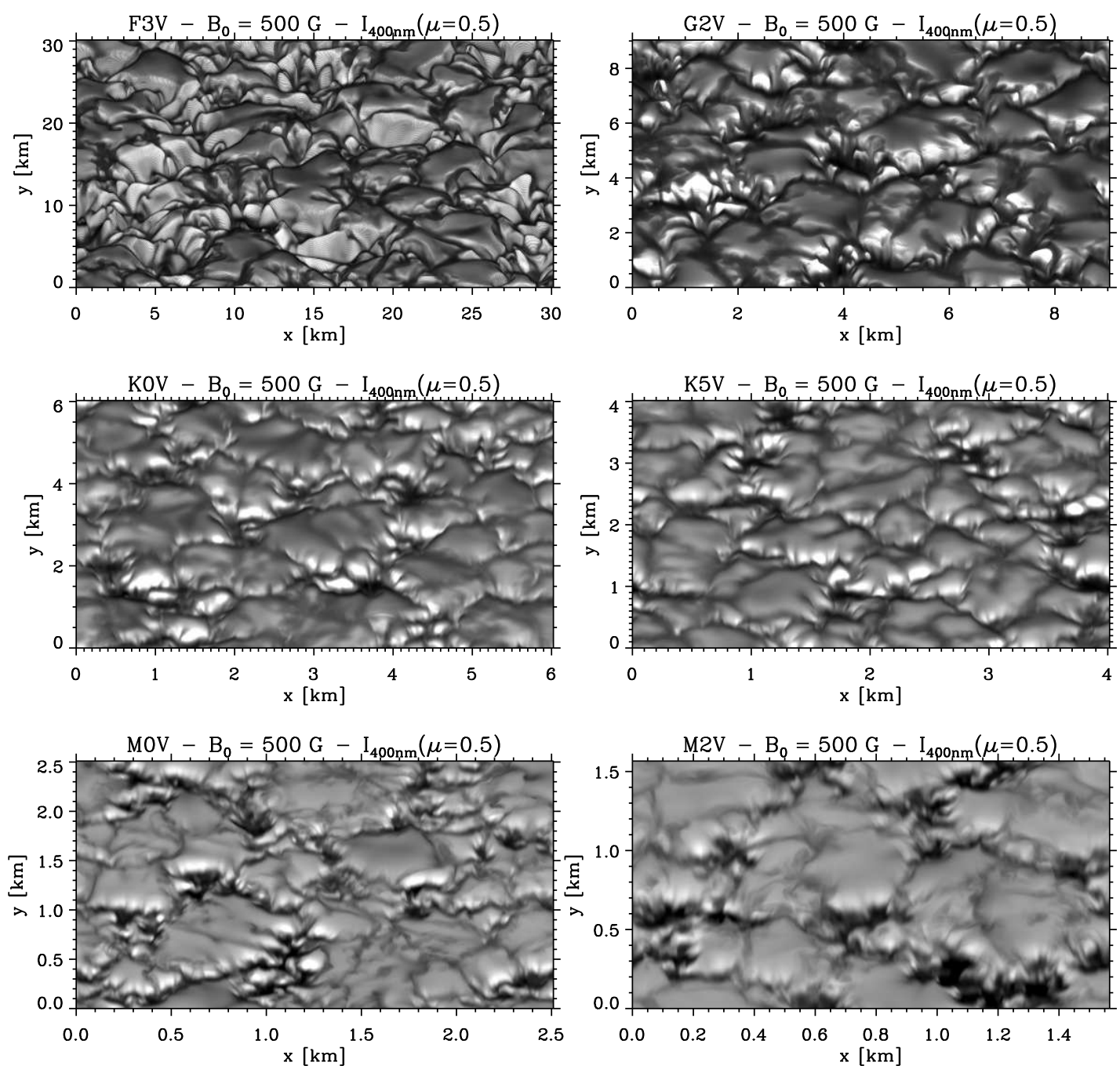

Figure 5.4: Continuum intensity at $400-410 \mathrm{~nm}$ leaving the simulation domains of the six simulations with $B_{0}=500 \mathrm{G}$ at an angle of $60^{\circ}$ (i. e. $\mu=0.5$ ). The snapshots are taken from the same simulation time step as in , e. g., Figs. 3.15, B.1, 3.16, and B.2.

ening results from a slightly decreased disc-centre intensity (not visible in the normalised representation of Fig. 5.5) and a slightly brightened limb. The larger pore-like structures are better visible in the vertically emerging light whereas the bright structures (stellar analogues of faculae) have a higher intensity and filling factor near the limb. In the F-star simulations, bright structures dominate: the disc centre and the limb both have a higher intensity with $500 \mathrm{G}$ than without magnetic field, the limb being more strongly brightened than the disc centre. In contrast, the M2V simulation is darker at all values of $\mu \geq 0.2$ in the $500 \mathrm{G}$ run, because of the high area fraction of dark micropores, the intensity reduction being stronger at disc centre than near the limb. In all cases, the resulting limb darkening is reduced in the magnetic runs. The impact of the magnetic field on the limb darkening is decreasing along the model sequence from hot to cool stars (cf. Fig. 5.6).

In Figure 5.6 the difference between the limb darkening of the non-magnetic run and 

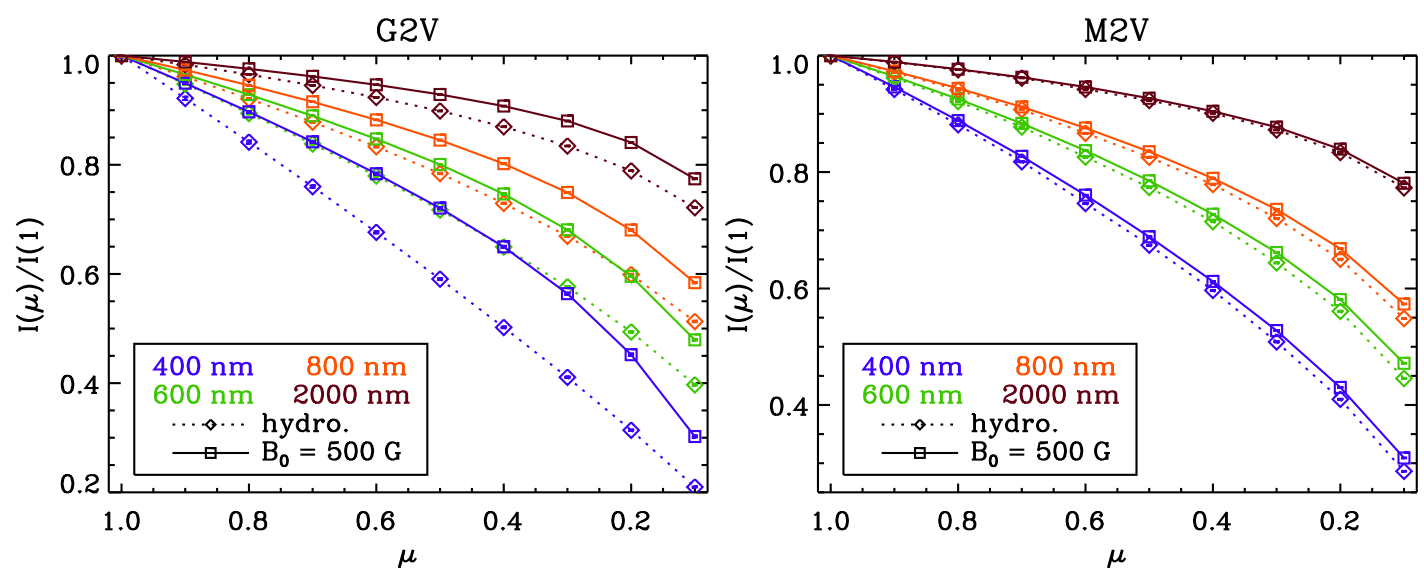

Figure 5.5: Limb darkening for the G2V (left) and M2V (right) simulations with $B_{0}=0 \mathrm{G}$ ( diamonds and dotted curves) and $500 \mathrm{G}$ (squares and solid curves) in narrow passbands near 400, 600, 800, and $2000 \mathrm{~nm}$ (for the same plots for the other four simulated stars see Fig. B.22). In both plots, the temporal scatter is indicated by (tiny) error bars.

the different magnetic runs is displayed for two simulated stars ( $\mathrm{F} 3 \mathrm{~V}$ and $\mathrm{K} 0 \mathrm{~V}$, for the other four stars, see Fig. B.23 in Appendix B). With $B_{0}=20 \mathrm{G}$, the F3V simulation has a stronger limb darkening than without magnetic field. Although this result is highly significant in terms of the errors given (deduced from the temporal scatter), it is not necessarily directly related to the magnetic field: the non-magnetic run shows a large-scale structure in the velocity field (cf. Fig 3.2) and the optical surface level (cf. Fig. 3.23), which is probably related to an oscillation excited by granulation. As the wavelength of this oscillation is comparable to the horizontal size of the simulation box, it is not clear if it is caused or modified by the periodic boundary conditions. This oscillation possibly leads to a slightly increased intensity near the limb (bright side-walls of protruding regions). In the magnetic runs, this large-scale structure has a lower amplitude or is not present at all in the analysed snapshots, which reduces the brightness near the limb. The magnetically caused brightening of the limb overcompensates this relative darkening in the $100 \mathrm{G}$ and $500 \mathrm{G}$ runs of the F3V star, but not in the $20 \mathrm{G}$ run, which shows a stronger limb darkening than the non-magnetic reference. With this one exception, the limb darkening is reduced in all magnetic simulations compared to the non-magnetic simulations. The effect is decreasing towards cooler stars and is strongly increasing with increasing $B_{0}$. Already at $100 \mathrm{G}$ average field, the deviation of the limb darkening from the non-magnetic case is significantly larger than the corresponding difference between the non-magnetic case and a 1D atmosphere (see Fig. 5.3). At $500 \mathrm{G}$ the deviation from the non-magnetic case is larger than the differences between different spectral types (see Fig. 5.2). This is an important result, e.g., for the detection and characterisation of extra-solar transiting planets (see Sect. 7.2).

Figure 5.7 shows the centre-to-limb variation of the rms continuum intensity contrast of the $500 \mathrm{G}$ and non-magnetic runs of the G2V and M2V simulations (for the corresponding figure for the other simulated spectral types, see Fig. B.24 in Appendix B). In the G2V simulation, the intensity contrast at the disc centre does not change considerably from non-magnetic to $500 \mathrm{G}$ run. The intensity contrast at $\mu<1$, however, is strongly 

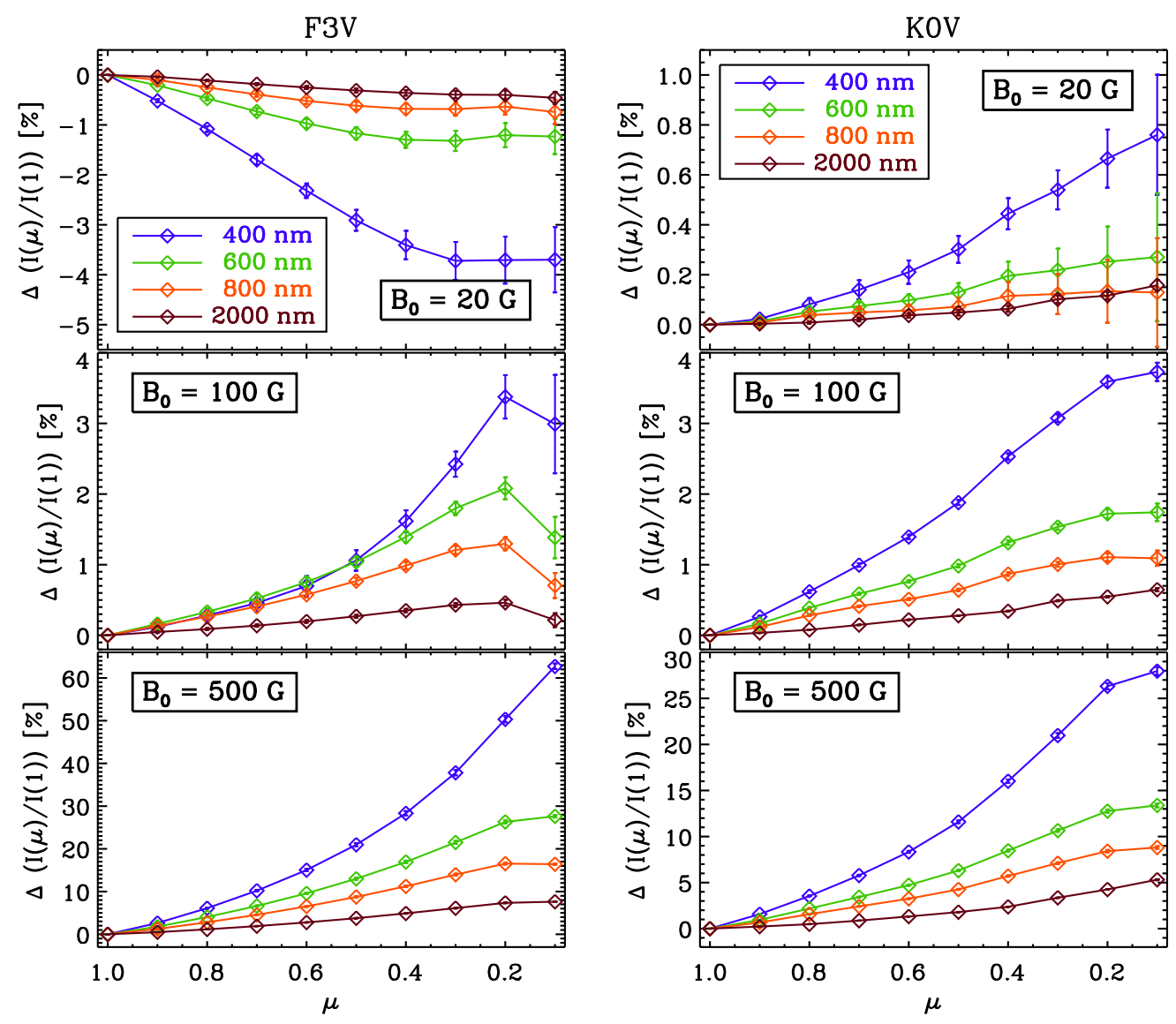

Figure 5.6: Difference of the limb darkening of the three magnetic simulations from the non-magnetic simulations of the F3V and K0V simulations (corresponding plots for the other stars are given in Fig. B.23). The colour code and abscissa scale are the same for all sub-plots. Note the different scale of the ordinates.

increased by the presence of the field. In the non-magnetic case, the contrast decreases monotonically from the disc-centre towards the limb, while, in the $500 \mathrm{G}$ runs, the contrast is highest near the $\operatorname{limb}(\mu \approx 0.3)$. A qualitatively similar result was obtained for the $\mathrm{K}$ stars (see Fig. B.24). This increasing intensity contrast towards the limb is caused by the the bright structures, which are brighter and have a higher area fraction near the limb ("faculae").

As discussed in Sect. 4.3.1, the bolometric intensity contrast at $\mu=1$ (disc centre) in the $\mathrm{M}$ stars is considerably higher in the $500 \mathrm{G}$ runs owing to the large area fraction covered by dark micropores. In the different passbands shown in Fig. 5.5, the contrast is enhanced by approximately a factor of two in the M2V star. The centre-to-limb variation of the contrast is less affected by the magnetic field than in other stellar types.

As in the non-magnetic case, the temporal scatter, ${ }^{3}$ which was used for an error esti-

\footnotetext{
${ }^{3}$ The analysis in this section was based on the six snapshots indicated for each simulation in Fig. 2.2. They span a time of roughly $10-15 \mathrm{~min}$, which is larger than the granule lifetime, but probably shorter than the time scale on which the micropores (cf. Sect. 4.3.3) and large-scale velocity patterns (mesogranulation) evolve.
} 

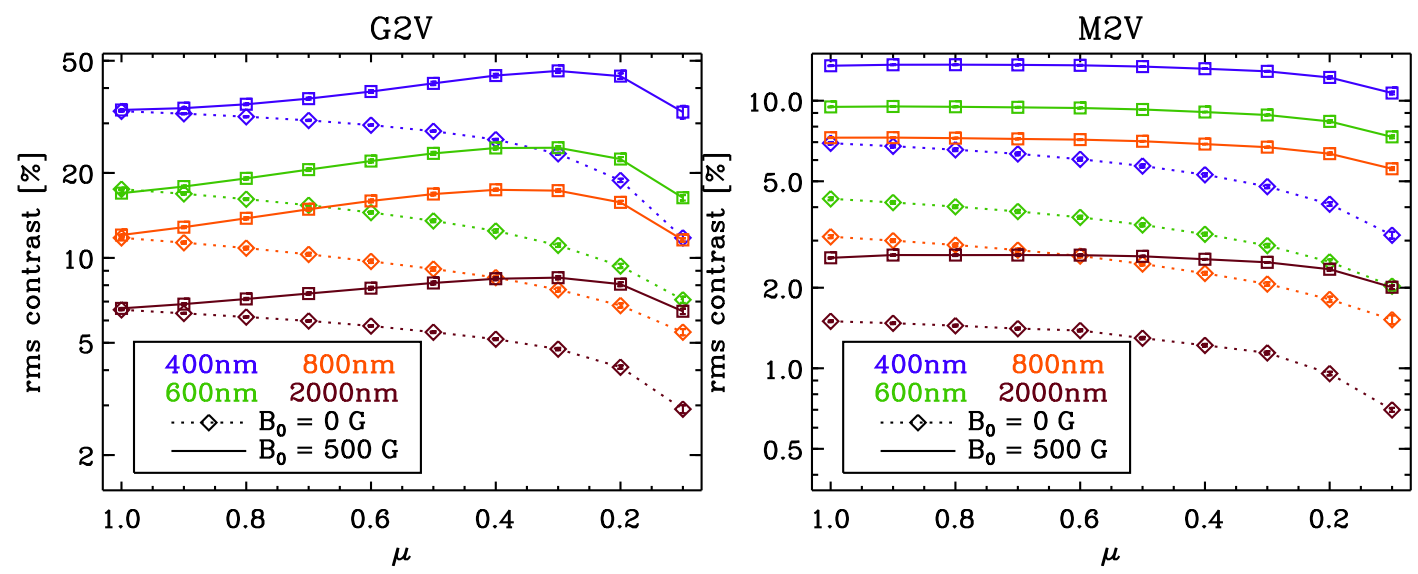

Figure 5.7: Centre-to-limb variation of the rms intensity contrast for the G2V (left) and $\mathrm{M} 2 \mathrm{~V}$ (right) simulations with $B_{0}=0 \mathrm{G}$ (diamonds and dotted curves) and $500 \mathrm{G}$ (squares and solid curves) in narrow passbands near 400, 600, 800, and $2000 \mathrm{~nm}$ (corresponding plots for the other four simulated stars are given in Fig. B.24). In both plots, the errors are indicated by (tiny) error bars. Note the logarithmic scale of the ordinates.
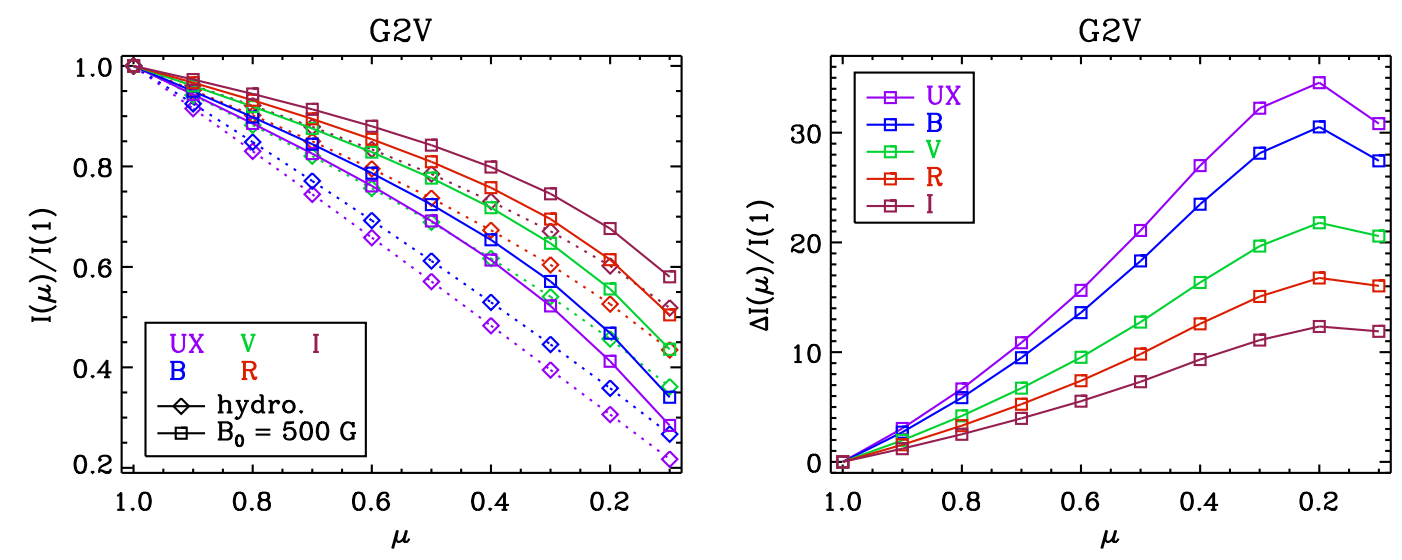

Figure 5.8: Limb darkening in the Johnson UX, B, V, R, and I passband filters (Johnson and Morgan 1951, Bessell 1990) in the G2V star (for corresponding plots for the F3V and M0V stars, see Fig. B.25). Left panel: Limb darkening of the non-magnetic (hydro.) and $500 \mathrm{G}$ runs. Right panel: Relative difference between both runs.

mate, is very small. The relative errors thus obtained are between $10^{-4}$ and $5 \cdot 10^{-3}$ for the limb darkening and between $5 \cdot 10^{-3}$ and $2.5 \cdot 10^{-2}$ for the contrast in most of the simulations. These errors are indicated by (often invisibly small) error bars in Figs. 5.5 - 5.7 and B.22 - B.24. Systematic errors might be considerably larger, as the 20 G-run result of the F3V star suggests (cf. Fig. 5.6).

For some of the $500 \mathrm{G}$ runs, the centre-to-limb variation in the Johnson UX, B, V, R, and I passband intensities were calculated with qualitatively similar results. The resulting limb darkening curves for the G2V star are given in Fig. 5.8. The corresponding plots for the F3V and M0V star are shown in Fig. B.25 in Appendix B. 



\section{Synthetic spectral lines}

\subsection{Line synthesis and stellar disc integration ${ }^{1}$}

In order to quantify the effect of the $3 \mathrm{D}$ structure on spectral lines, we chose as three representative lines: an infrared $\mathrm{Ti}$ I line at $2223 \mathrm{~nm}$ and two optical Fe I lines at 616.5 and $617.3 \mathrm{~nm}$, which are present in all stars of our effective temperature range, although the titanium line is weak in the two hottest models. These lines are relatively isolated (unblended) and we possess high-resolution archive spectra of F- and G-type main-sequence stars covering spectral ranges containing the two iron lines (see Sect. 6.2.4). All lines are magnetically sensitive and are suitable for measuring stellar magnetic fields (Anderson et al. 2010, Johns-Krull et al. 2004, and several follow-up papers). This will become relevant in the third paper of this series, in which the impact of the magnetic field on convection and spectral lines will be discussed.

The lines were calculated with the line synthesis code SPINOR (Frutiger 2000) for six 3D snapshots for each simulation. We considered snapshots 2000 simulation time steps $\delta t$ apart (about $5-7$ minutes stellar time).

For comparison with spatially unresolved stellar observations, we integrated the light over a constructed stellar disc. This integration can be done semi-analytically by a convolution for rigid-body rotation and homogeneous surface properties (Nordlund and Dravins 1990b, Ludwig 2007). Real stars, however, often rotate differentially or have large-scale inhomogeneities such as star spots. In order to cover this general case, we implemented a numerical stellar-disc integration.

The locally averaged line profile $I_{0}(\lambda, \mu)$, which is generally a function of wavelength $\lambda$ and angle $\theta$ (with $\cos \theta=: \mu$ ) between line of sight and surface normal, is only calculated for a limited number $N_{\mu}$ of reference values $\mu_{j}$. One considers $I_{0}(\lambda, \mu)$ to be represented by the profile $I_{0}\left(\lambda, \mu_{j}\right)$ within a small interval $\tilde{\mu}_{j} \geq \mu>\tilde{\mu}_{j+1}\left(j=0,1, \ldots, N_{\mu}-1\right)$,

${ }^{1}$ This section has been published as Sect. 5.1 in Beeck et al. (2013b).

Table 6.1: Line parameters.

\begin{tabular}{lrr}
\hline \hline Species & $\lambda_{\text {rest }}[\mathrm{nm}]$ & $E_{i}[\mathrm{eV}]^{\mathrm{a}}$ \\
\hline $\mathrm{Fe}_{\mathrm{I}}$ & 616.536 & 4.143 \\
$\mathrm{Fe}_{\mathrm{I}}$ & 617.333 & 2.223 \\
$\mathrm{Ti}_{\mathrm{I}}$ & 2223.284 & 1.739 \\
\hline
\end{tabular}

a $E_{i}$ is the excitation potential of the lower level of the transition 


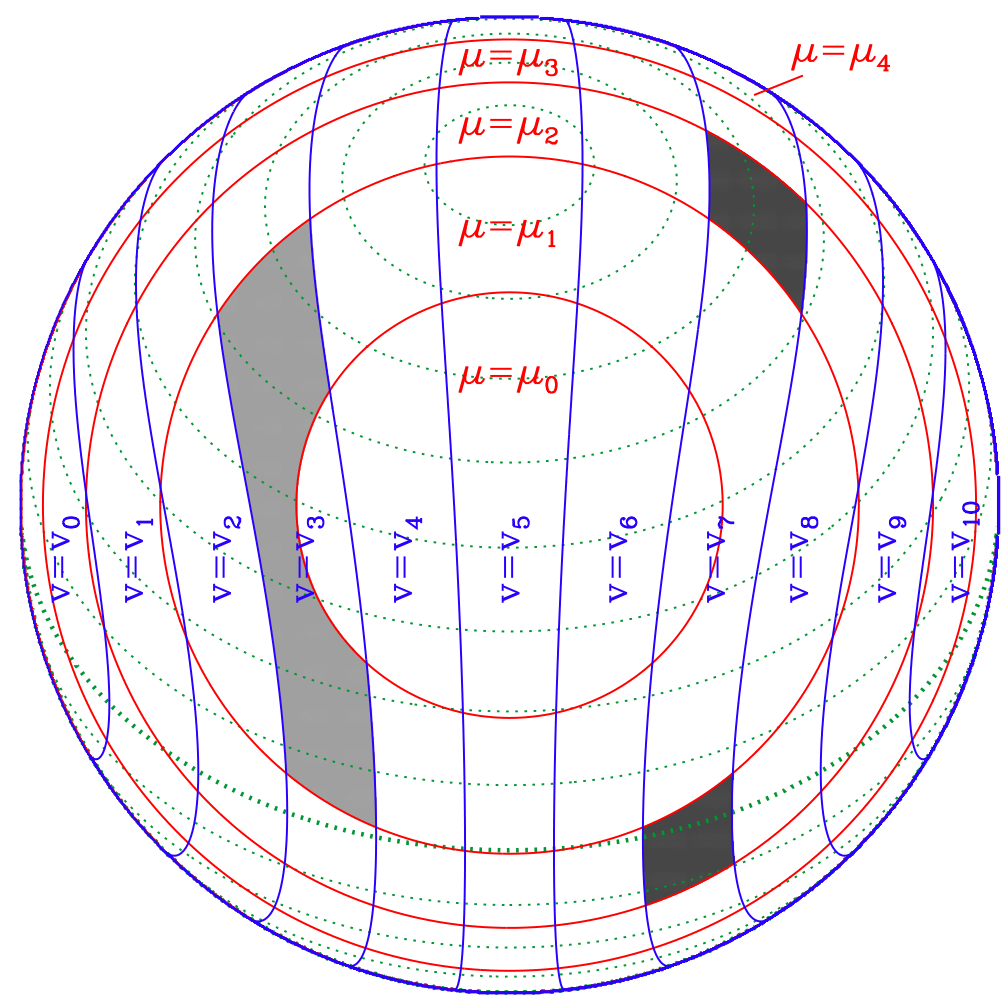

Figure 6.1: Sketch of a stellar disc decomposed into areas of nearly-constant velocity (" $v$-stripes") and surface normal direction (" $\mu$-rings") for a star with differential rotation in latitude. Red circles indicate the limits between the $\mu$-rings, blue curves indicate the limits between $v$-stripes, the dotted green curves show constant latitude circles on the stellar sphere (in steps of $\left.10^{\circ}\right)$. Two bins $\left(w_{1,3}\right.$ and $\left.w_{2,7}\right)$ are filled in different shades of grey for illustration. The shown star has an inclination of $45^{\circ}$ and rotates differentially with twice the solar equator-to-pole shear ( $\alpha=0.4)$. In this sketch we used $N_{\mu}=5$ and $N_{v}=11$. For the numerical stellar-disc integrations considered in Sect. 6.2.3 and 6.2.4 we used $N_{\mu}=10$ and $N_{v}=51$.

where $\tilde{\mu}_{j}>\mu_{j}>\tilde{\mu}_{j+1}$. On the stellar disc, these $\mu$ intervals correspond to concentric rings (and a central disc). To include rotation (differential or rigid), the constructed stellar surface is additionally divided into "velocity stripes", which are the projected areas of regions with a line-of-sight component of the rotation velocity in a given interval $\tilde{v}_{k} \leq$ $v<\tilde{v}_{k+1}\left(k=0,1, \ldots, N_{v}-1\right)$. Figure 6.1 shows a sketch of a stellar disc with $N_{\mu}=5$ $\mu$-rings and $N_{v}=11 v$-stripes. To obtain the disc-integrated line profile, $F(\lambda)$, we replace the wavelength dependence of $I_{0}$ by a (mathematically equivalent) line-of-sight-velocity dependence and obtain:

$$
F(v)=\sum_{j=0}^{N_{\mu}-1} \sum_{k=0}^{N_{v}-1} w_{j k} I_{0}\left(v+v_{k}, \mu_{j}\right),
$$

where each weight $w_{j k}$ corresponds to the projected area on the stellar disc with $\tilde{\mu}_{j} \geq \mu>$ $\tilde{\mu}_{j+1}$ and $\tilde{v}_{k} \leq v<\tilde{v}_{k+1}$ normalised by the total projected disc area. Interval limits were chosen such that the reference values $\mu_{j}$ and $v_{k}$ are centred in the intervals. 
For the differential rotation we here use the simple law

$$
\Omega=\Omega_{\mathrm{eq}} \cdot\left(1-\alpha \sin ^{2} \theta\right) .
$$

The differential rotation parameter $\alpha$ is positive for solar-like differential rotation $\left(\alpha_{\odot} \approx\right.$ 0.2 at the surface, Howard and Harvey 1970) and negative for anti-solar differential rotation. With the current implementation of the numerical integration, we are able to cover the range $-0.5 \leq \alpha \leq+1.0$, which is much wider than the range of today's observed and predicted values of $\alpha$ in stars.

It is computationally expensive to use many $\mu$-rings because each value of $\mu_{j}$ requires a separate 3D line synthesis. In their pioneering work, Nordlund and Dravins (1990b) used $N_{\mu}=3$ and included rigid-body rotation by convolution. Here, we used $N_{\mu}=10$ with $\mu_{j}=1.0,0.9, \ldots, 0.1$.

In contrast, the number of $v$-stripes has only a minor impact on the computational expense and thus can be chosen almost arbitrarily high. In a few test cases with rigid-body rotation, the relative error between the semi-analytical result and the numerical method was found to be smaller than $10^{-4}$ for $N_{v} \geq 50$, which is lower than the typical statistical error due to the limited number of simulation snapshots. For the disc integration presented in this paper, we used $N_{v}=51$. The numerical values of $v_{k}$ scale linearly with $v_{\text {rot }} \sin i$ (the line-of-sight component of the rotational velocity of the stellar equator; $i$ is the inclination of the rotation axis with respect to the line of sight). Once calculated, the $N_{\mu} \times N_{v}$ weights $w_{j k}$ for a given combination of $i$ and $\alpha$ can be used for the integration for any rotational velocity $v_{\text {rot }}$ and given local profiles $I_{0}\left(v, \mu_{j}\right)$.

\subsection{Spectral lines without magnetic fields ${ }^{2}$}

\subsubsection{Spatially resolved line profiles}

Figure 6.2 shows the vertically $(\mu=1)$ emerging spectrum of the Ti I line at $2223 \mathrm{~nm}$ for two locations (one in an upflow and one in a downflow region) in a snapshot of the K0V simulation. The depth of the line depends on temperature and temperature gradient; it is smaller in the downflow region, where the temperature gradient is lower. While the wings of this line form at $-1<\log \tau_{\mathrm{R}}<0$ in the $\mathrm{K} 0 \mathrm{~V}$ model, the line core forms at $-2.5<\log \tau_{\mathrm{R}}<-1$. Hence, the depth-dependence of the flow velocity entails line asymmetries: in upflow regions, the whole line is blueshifted, but as the flow decelerates with height, the line core is less strongly shifted than the wings. Analogously, the line wings originating in a downflow accelerating with depth are more strongly redshifted than the line core.

\subsubsection{Centre-to-limb variation of line profiles}

Figure 6.3 illustrates the centre-to-limb variation of the Fe I line profile at $617.3 \mathrm{~nm}$ averaged over six snapshots of the F3V simulation box. The convective blueshift and line asymmetry are largest at disc centre and decrease towards the limb. For the Sun, this

\footnotetext{
${ }^{2}$ This Section has been published as Sects. $5.2-5.5$ in Beeck et al. (2013b).
} 

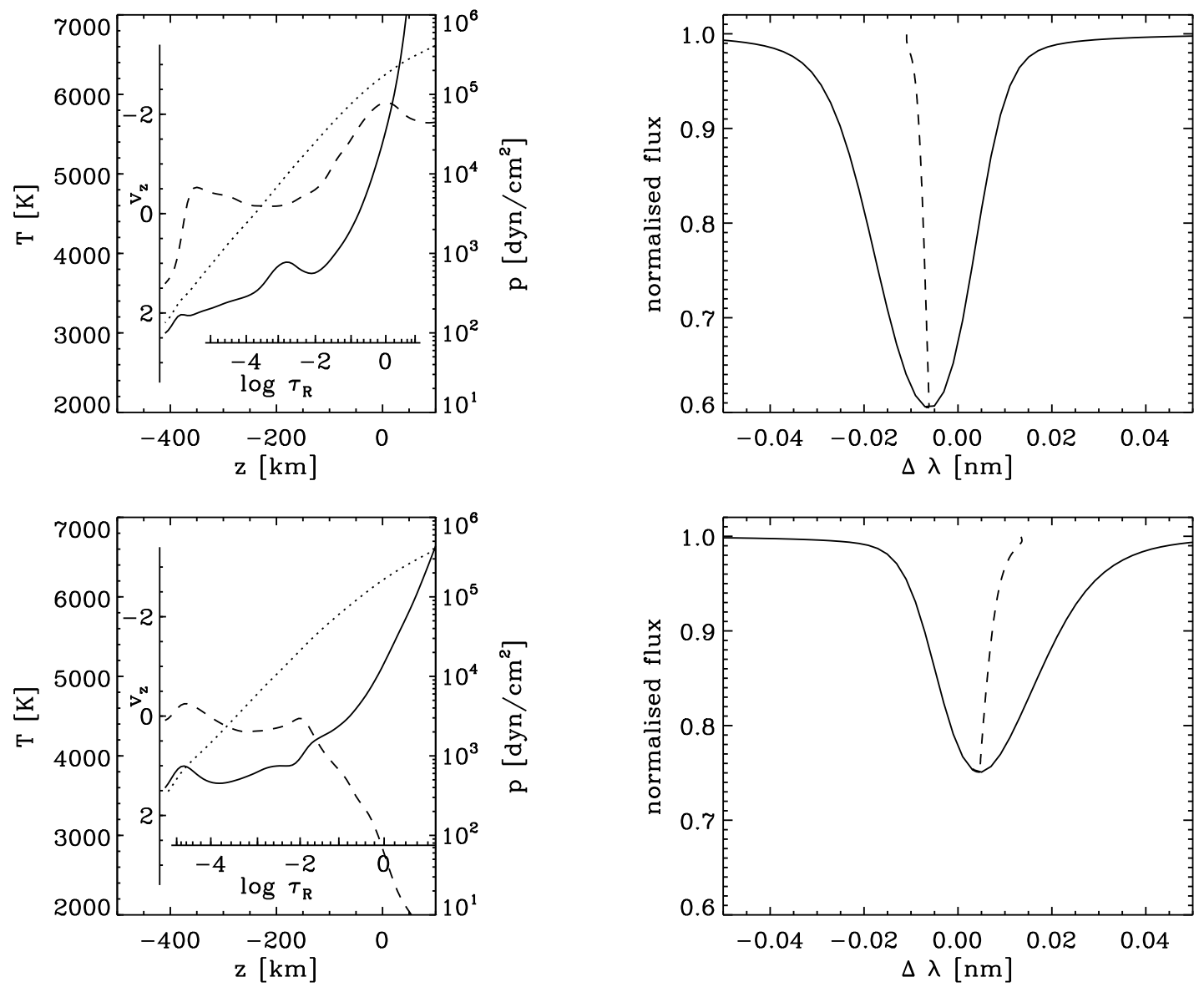

Figure 6.2: Synthetic line profiles (Ti I at $2223 \mathrm{~nm}$ ) of single vertical rays of the K0V-star simulation in an upflow region (upper panels) and in a downflow region (lower panels). Left panels: Temperature (solid), pressure (dotted), and vertical component of the fluid velocity (dashed) along the vertical ray. Right panels: Profiles of the Ti I line at $2223 \mathrm{~nm}$ obtained with SPINOR for the corresponding vertical rays $\left(\Delta \lambda=\lambda-\lambda_{\text {rest }}\right.$; the dashed lines give the bisectors of the profiles).

effect has been demonstrated in many observational studies and is referred to as the "limb effect" (for a review, see Dravins 1982). It is a consequence of the correlation between intensity and velocity: at disc centre, the line-of-sight velocity corresponds to the vertical flow velocity. As upflows (granules) are bright and have a higher area fraction, they contribute more to the average line profile provided that the line depth in up- and downflows is comparable. Therefore, most lines are convectively blue-shifted at disc center. Near the limb, the line-of-sight velocity is dominated by the horizontal flow component, which has no correlation between its direction and temperature. This results in a more symmetrical line profile with a less shifted line core near the limb. The line wings are, however, somewhat more blue-shifted (near wings) or less red-shifted (extreme wings) near the limb than at the disc centre. As the line wings originate close to the optical surface, this is probably caused by very bright front edges of the granules in this star.

The upper panels of Figure 6.4 show the variation of equivalent width and FWHM 

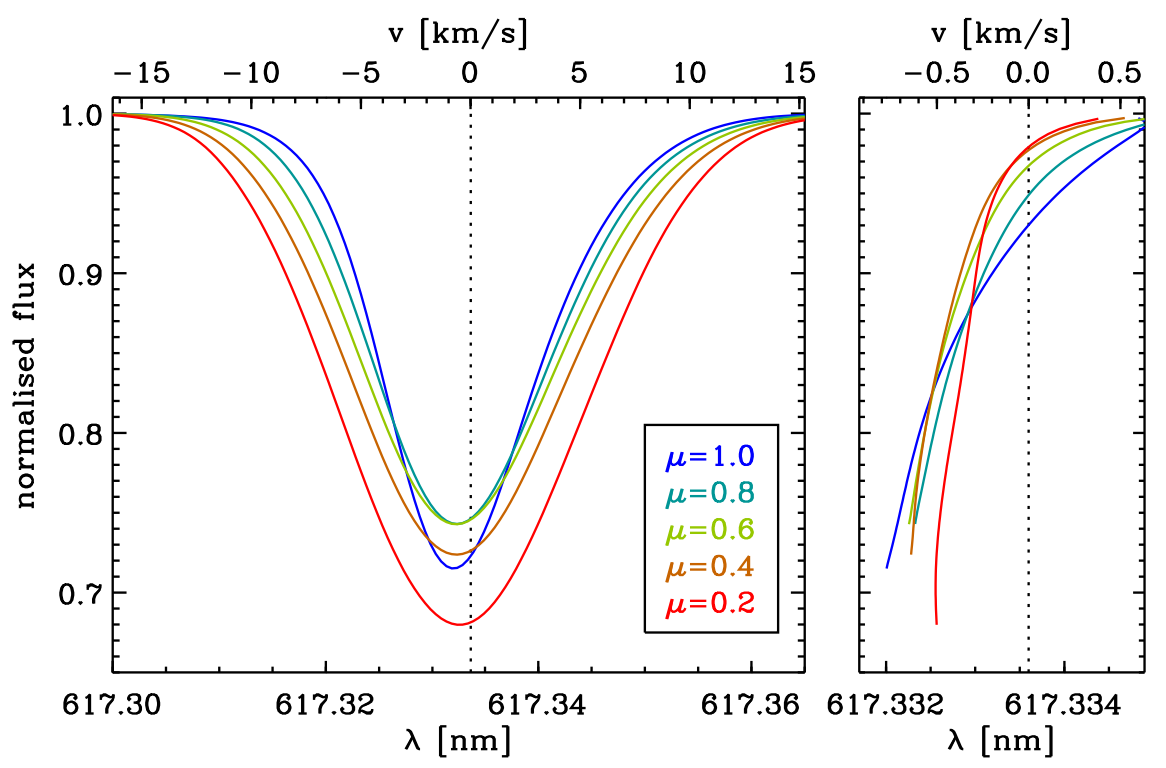

Figure 6.3: Centre-to-limb variation of a sample line profile. Left panel: line profile obtained from the F3V simulation (Fe I line at $617.3 \mathrm{~nm}$; average over six snapshots) for various values of $\mu=\cos \theta$. Right panel: bisector variation of the profile; same color code and ordinate labels as left panel.

with $\mu$ for the same line as in Figure 6.3, but for all our simulations. For the cooler simulations, the equivalent width of this line decreases towards the limb as a consequence of the decreasing temperature gradient with respect to optical depth along the inclined optical path. In the two hottest models, however, the line is stronger (higher equivalent width) near the limb (cf. Fig 6.3), because of the high temperature in the lower photospheres: the excitation potential of $2.2 \mathrm{eV}$ of the lower level of the transition is relatively low and this level becomes depopulated as the temperature rises significantly above $7000 \mathrm{~K}$. In addition, at this temperature, the first ionisation of iron sets in, so that the abundance of Fe I drops rapidly with temperature.

The FWHM of the spectral line is growing in all stars towards the limb as a consequence of the increasing contribution of the horizontal flow velocity to the line-of-sight velocity: the rms of the horizontal velocity is up to about three times as high as the rms of the vertical velocity in the photosphere and above (cf. Fig. 3.4). This leads to a stronger Doppler broadening of the line at low values of $\mu$.

The lower panels of Figure 6.4 show the Doppler shifts of the line wings (5\% of total line depth) and the line core. The line wings are slightly blueshifted for most stars and show a weak dependence on $\mu$. However, for the F3V star, the line wings are strongly redshifted at the disc centre and un-shifted near the limb. This indicates that, at disc centre, the downflow regions contribute considerably to the resulting integrated profile of this line in spite of their lower continuum intensity and area fraction. As discussed above, this line becomes weak due to the high photospheric temperatures and since upflows are much hotter than downflows, the upflows contribute very little to the average line profile. This effect vanishes near the limb, where the line-of-sight velocities correspond rather to the horizontal component of the flows and where the line forms higher in the atmosphere, 

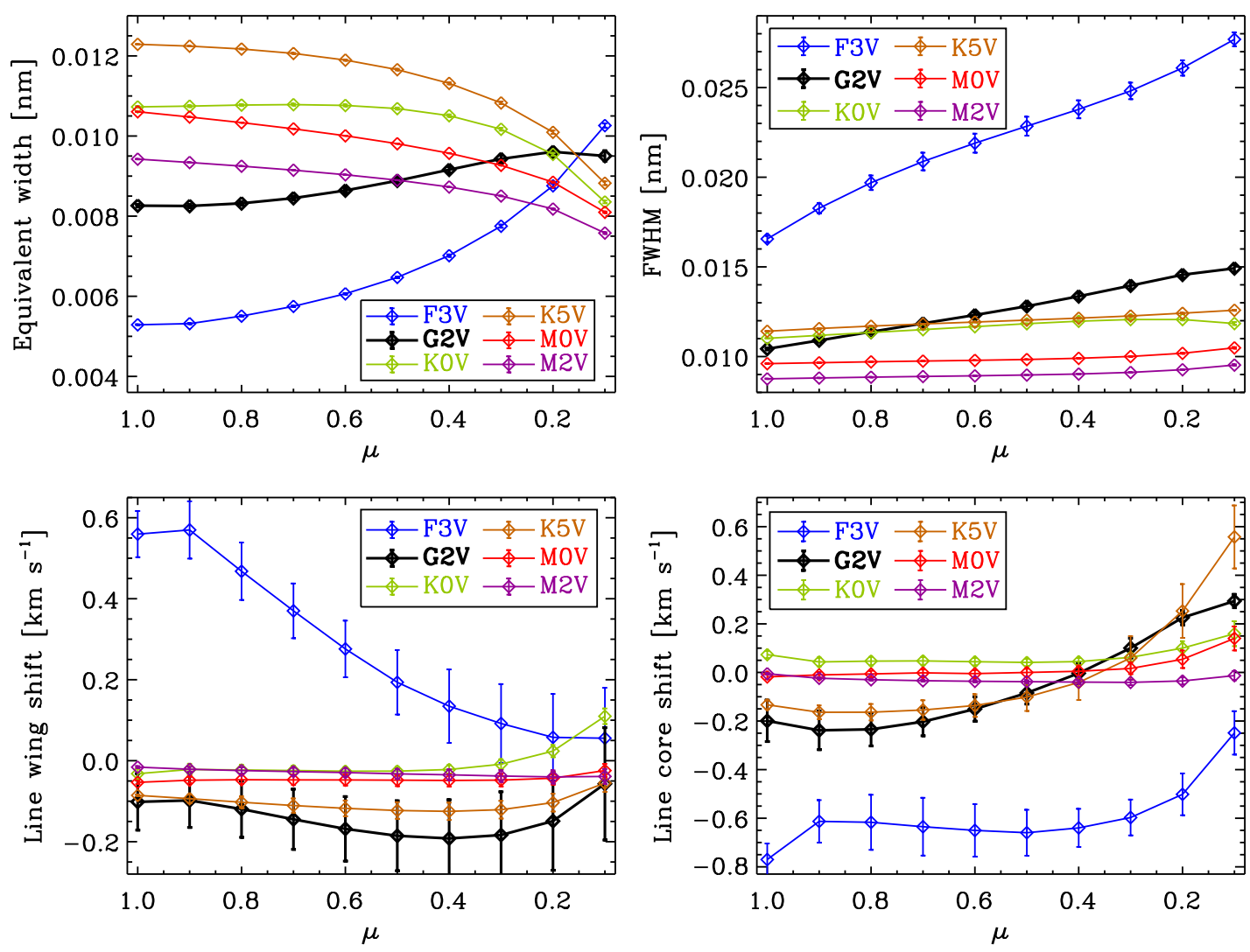

Figure 6.4: Centre-to-limb variation of the $\mathrm{Fe}$ I line at $617.3 \mathrm{~nm}$. Top panels: Variation of equivalent width (right) and FWHM (left) of the line as functions of $\mu$. Bottom panels: Convective Doppler shift (translated into line-of-sight velocity) of the line wings (right; $5 \%$ level of max. line depth) and the line core (left) as functions of $\mu$ (positive $=$ redshift). The gravitational redshift is neglected.

i.e. at lower temperature.

The line cores of all stars except M2V show a pronounced increasing redshift (or decreasing blueshift) at decreasing $\mu$. The fact that some line cores are redshifted near the limb is due to a statistical bias owing to reversed granulation: receding flows are more often seen in front of hotter gas above intergranular lanes and approaching flows are more often seen in front of the cooler gas above granules (e. g., Asplund et al. 2000). This effect is irrelevant for the line wings, as their formation height is closer to the optical surface.

The convective blueshift and its centre-to-limb variation are very important for highprecision radial velocity measurements (e.g. for exoplanet detection and characterisation). As this section has shown, the many different mechanisms that determine the convective blueshift for individual spectral lines can only be reproduced by comprehensive 3D calculations (also see Ramírez et al. 2009, Allende Prieto et al. 2013). 

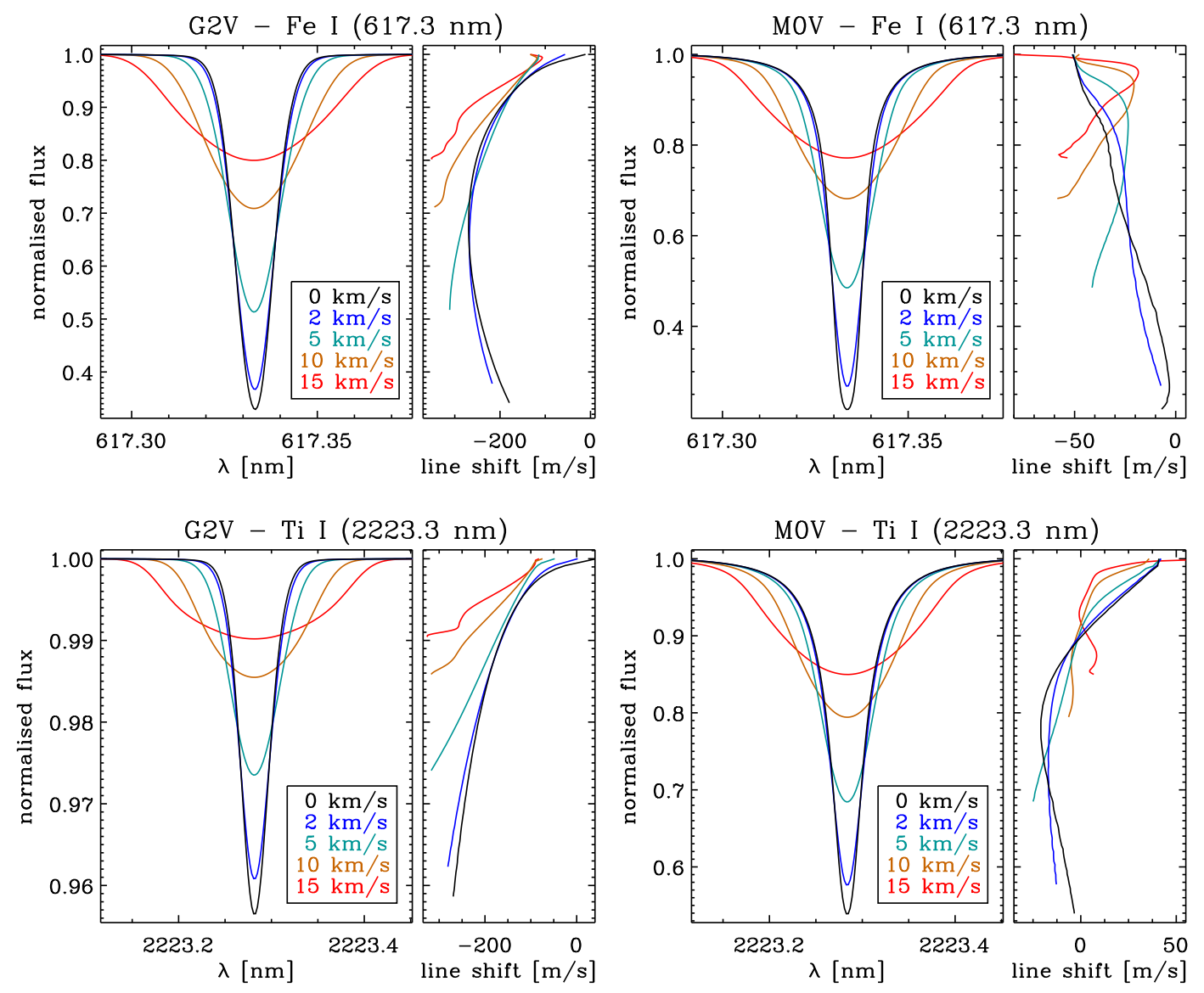

Figure 6.5: Rotational broadening and line distortion. Upper panels: rotational broadening of the $\mathrm{Fe}_{\mathrm{I}}$ line at $617.3 \mathrm{~nm}$ for the G2V (left) and M0V (right) simulations. The narrow right sub-plots of each plot show the bisectors of the lines (same colour code and ordinate labels as other sub-plot). Lower panels: same as upper panels, but for the Ti I line at $2223 \mathrm{~nm}$. In all cases, we used solar differential rotation together with five different rotation rates.

\subsubsection{Disc-integrated line profiles}

Using the numerical method outlined in Sect. 6.1, we calculated disc-integrated profiles of the three spectral lines listed in Table 6.1 for six snapshots of each of our six simulations.

Figure 6.5 shows the profiles of two lines (Fe I at $617.3 \mathrm{~nm}$ and $\mathrm{Ti}$ I at $2223.3 \mathrm{~nm}$ ) for the solar $(\mathrm{G} 2 \mathrm{~V})$ and the M0V simulations for solar-like differential rotation $(\alpha=0.2$, Eq. (6.2)) and various values of $v_{\text {rot }} \sin i$ (i. e., the projection of the equatorial rotation velocity onto the line of sight). As we have discussed in the previous section, the convective flows and their correlation with temperature generally cause line asymmetries and shifts (with respect to the rest wavelength of the line). These effects are visible in the disc-integrated profiles as well and best illustrated by line bisectors, which are generally curved and shifted. Stellar rotation broadens the spectral line. The line shift due to rotation is symmetric with respect to the projected rotation axis on the stellar disc (see Fig. 6.1) and does therefore not produce an additional line asymmetry. However, asymmetries of the local profiles (due to convection) can be modified by rotation in a non-trivial fashion. 


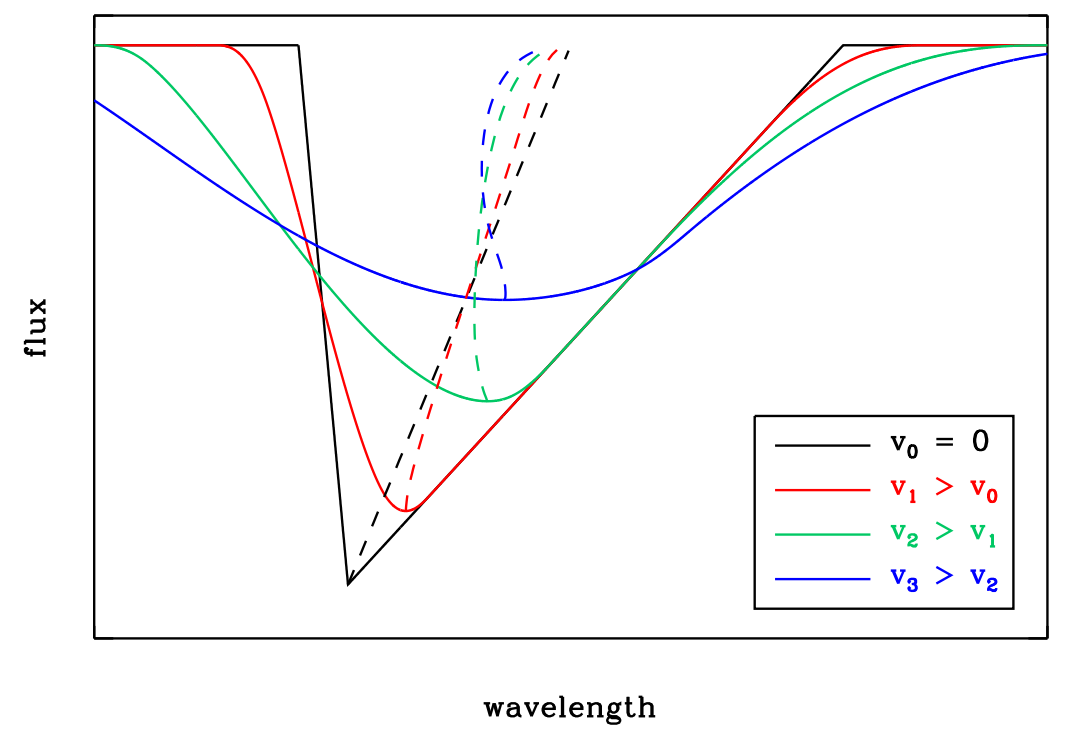

Figure 6.6: Illustration of line broadening and bisector modification by rotation. The black solid line shows an artificial line profile with a straight (but inclined) bisector (black dashed curve). The coloured curves were obtained by convolving the black curve with a (rigid-body) rotation profile of different width, simulating different velocities. Note that the bisectors (dashed curves) are modified by the convolution although the rotation profile is perfectly axis-symmetric.

This is illustrated in Figure 6.6: here, a $\mu$-independent artificial line profile is convolved by a rotation profile to mimic stellar rigid-body rotation $(\alpha=0)$. The bisector depends on rotation velocity and evolves from a straight line into curved $\mathrm{C}$ - and S-shapes when the rotation velocity is increased.

Figure 6.7 shows the dependence of the bisector of the Fe I line at $617.3 \mathrm{~nm}$ on the differential rotation parameter $\alpha$ as defined in Eq. (6.2) and on the inclination $i$ of the rotation axis of the star at a rotation speed of $v_{\text {rot }} \sin i=5 \mathrm{~km} \mathrm{~s}^{-1}$. When $\alpha$ is varied, $i=60^{\circ}$ is fixed, when $i$ is varied, $\alpha=0.2$ is fixed. While the impact of $i$ on the bisector shape is very subtle, the effect of $\alpha$ is more pronounced. The effect is similar in magnitude for all inclinations between 15 and $90^{\circ}$ at constant $v_{\text {rot }} \sin i$. In contrast, we find that the effect of inclination is proportional to $|\alpha|$ and thus vanishes for rigid-body rotation $(\alpha=0)$ as expected. The dependence of the bisector shape on $\alpha$ and $i$ can be explained by the different contribution of differently shifted and inclined projected surface elements of the star. With our numerical method (see Sect. 6.1 and Fig. 6.1), the weights $w_{j k}$ change with $\alpha$ and (if $\alpha \neq 0$ ) with $i$. Similarly a continuous contribution function $w(\mu, v)$ would depend on both parameters. This implies that, at least for inactive stars with homogeneous convective surface structure, the investigation of the bisector shape of individual lines can yield constraints on the rotational velocity and the differential rotation parameter. 

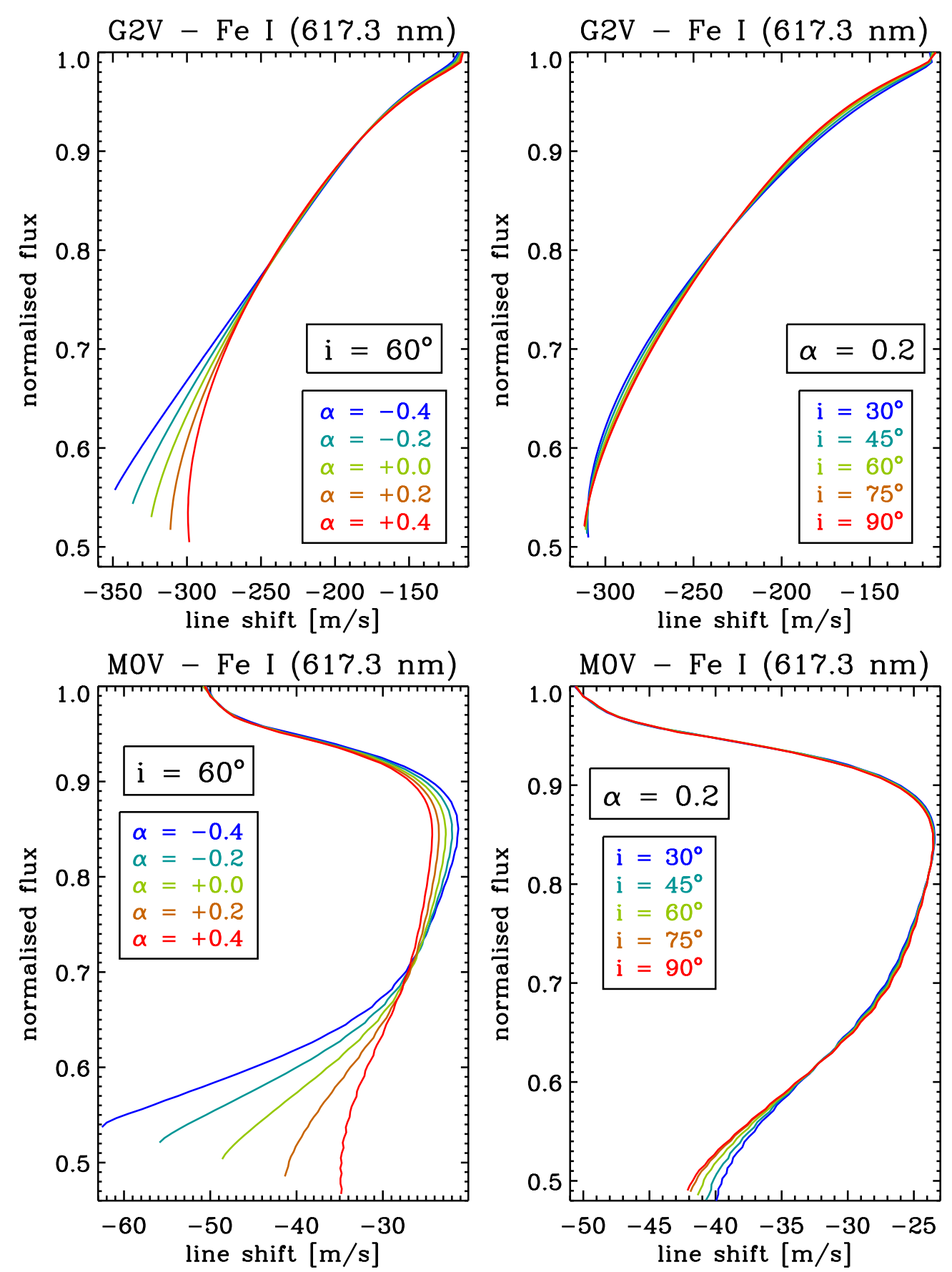

Figure 6.7: Bisector variation with inclination $i$ and differential rotation parameter $\alpha$ of the Fe I line at $617.3 \mathrm{~nm}$ for the G2V and M0V simulations. For the variation of $\alpha$ (left panels) the inclination was fixed at $i=60^{\circ}$; for the variation of $i$ (right panels) the differential rotation parameter was fixed at $\alpha=+0.2$. For all calculations $v \sin i=5 \mathrm{~km} \mathrm{~s}^{-1}$ was used.

\subsubsection{Comparison to observational data}

For a preliminary comparison of our disc-integrated line profiles, we considered three $\mathrm{F}$ stars from a set of observations of main-sequence stars obtained with the CES spectro- 
graph mounted on the 3.5-m telescope at Calar Alto observatory (Reiners 2006, Ammlervon Eiff and Reiners 2012).

Figure 6.8 shows a comparison of disc-integrated synthetic spectra with their observational counterpart. As we have only a limited number of spectral types and only one set of elemental abundances, the depth of the synthetic spectral line did not match the observed ones and was multiplied by a factor in order to facilitate a better comparison between model and observation. For the unknown inclinations, we assumed $i=60^{\circ}$ as the "average case". The projected rotational velocity $v_{\text {rot }} \sin i$ and the differential rotation parameter $\alpha$ was set to the values published by Ammler-von Eiff and Reiners (2012). The observed data have typical signal-to-noise ratios of several hundred and a spectral resolving power of $R \approx 230,000$. The instrumental broadening was mimicked by a convolution of the synthetic spectra with a gaussian. In general, the synthetic spectra provide a good representation of the observations. Some deviations are likely due to the mismatch in effective temperature, surface gravity, and chemical composition between stars and simulations as well as to magnetic activity and surface inhomogeneities in the observed stars, which are not taken into account so far.

The preliminary comparison to observed data in this section merely serves as an illustration and an outlook. A more detailed analysis of observational data is beyond the scope of this paper and will follow in a subsequent paper.

\subsection{Effects of the magnetic fields}

The three lines investigated here are sensitive to the Zeeman effect (the effective Landé factors, $g_{\text {eff }}$, are $0.69,2.5$, and 1.66, for the Fe I line at $616.5 \mathrm{~nm}$, the Fe I line at $617.3 \mathrm{~nm}$ and the Ti I line at $2223.3 \mathrm{~nm}$, respectively, see VALD Data Base, Piskunov et al. 1995, Kupka et al. 1999). If the magnetic field had no influence on the thermodynamic structure of the photospheric layers of the stars (which, for lack of information, is often assumed in measurements of stellar magnetic fields), one would expect the Stokes-I components of the lines to roughly resemble superpositions of un-split lines (originating in non-magnetic regions) and split or strongly broadened lines (originating in the magnetic flux concentrations). The weight of the two components is then just the area fraction of magnetised regions. Some observers refine this technique and take into account not just two but several components of different field strengths. This method has been successfully applied to fit observed spectral lines with model spectra, where a two-component model did not suffice (Shulyak, submitted).

However, as our simulations (as well as observations of the Sun) show, the magnetic field has a significant impact on the local thermodynamics and as all spectral lines are also sensitive to the pressure and temperature along the optical path, the situation can become far more complicated. In the partially evacuated Wilson depressions, the layer of line formation (which is roughly at $-3.0 \lesssim \log \tau_{\mathrm{R}} \lesssim-0.5$ for the lines investigated) is geometrically deeper and (at least for F-, G- and K-stars) often at a higher temperature (and a lower temperature gradient) in small-scale flux concentrations. The higher temperature can lead to the ionisation of the line-producing atomic species and thus to a reduced line opacity for lines of neutral atoms as considered here. Moreover, the magnetic and nonmagnetic parts of the photosphere can have statistically different velocity distributions, 

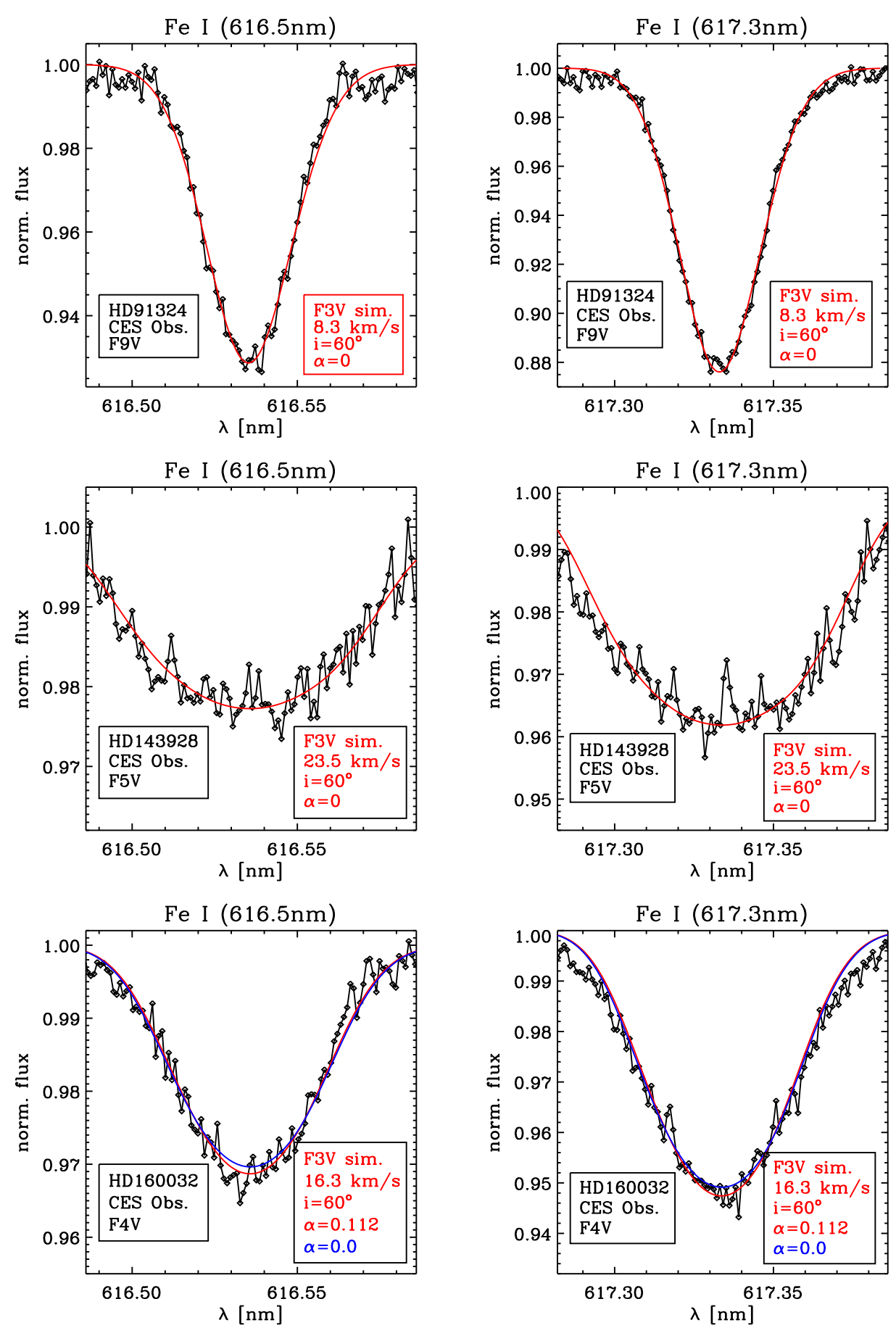

Figure 6.8: Profiles of the two Fe I lines of three F stars (black lines and diamonds) in comparison to disc-integrated profiles from the F3V simulation (red curves). For the rotational broadening of the synthesised lines, an inclination of the rotation axis of $i=60^{\circ}$ was assumed. For a better comparison, the line profiles were multiplied by a factor such that the depth of the line core come into agreement. For the projected rotational velocities $v_{\text {rot }} \sin i$ and differential rotation parameter $\alpha$ the values by Ammler-von Eiff and Reiners (2012) were used; for comparison, the blue curve in the bottom panels shows a discintegrated profile with the same $v_{\text {rot }} \sin i$ but $\alpha=0$. 


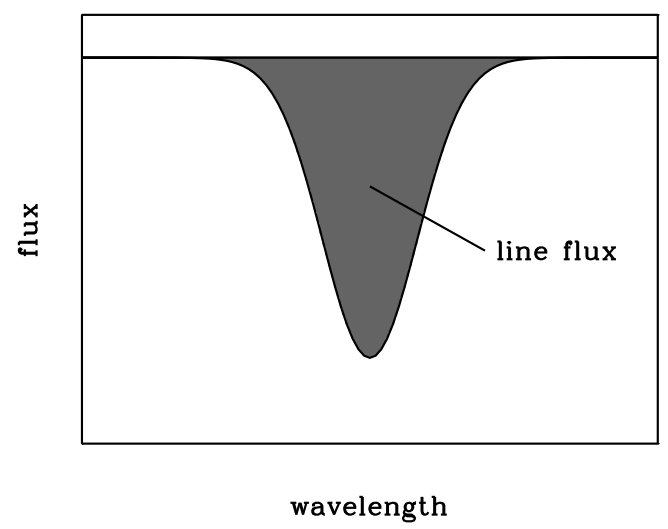

Figure 6.9: Illustration of the definition of (spectral) line flux in analogy to the flux of emission lines. As only absorption lines are considered here, the quantity called line flux in what follows is, strictly speaking, the modulus of the (negative) absorption line flux.

which means that the shift and broadening of lines due to the Doppler effect is different (and has probably a different $\mu$-dependence).

Even if a line is fairly insensitive to temperature, the differently bright parts of the stellar surface contribute different amounts of flux to the spectrum. For the analysis of a spectral absorption line, the amount of flux missing with respect to the non-normalised continuum gives the weight of a profile in the composite profile. In analogy to the flux of emission lines, this quantity will be called (spectral) line flux in the following sections. As only absorption lines are considered here, just the modulus of the line flux is of importance. Thus, all line fluxes in the following appear as positive quantities. Figure 6.9 illustrates this definition of the spectral line flux.

As will be shown in Sect. 6.3.4, even for the relatively weak average magnetic fields of up to $500 \mathrm{G}$ considered in this thesis, the local changes in the thermodynamic structure have a stronger impact on the three spectral lines investigated than the Zeeman effect for F- and G-type stars. In the following three sections, the influence of these local modifications is illustrated in spatially resolved "local" line profiles (Sect. 6.3.1), the centre-tolimb variation of the averaged line profiles (Sect. 6.3.2), and the disc-integrated profiles (Sect. 6.3.3).

\subsubsection{Spatially resolved spectra}

Figure 6.10 shows the local profiles of two spectral lines for four single vertical rays in one snapshot of the K0V simulation with $B_{0}=500 \mathrm{G}$. Point 1 is located in a non-magnetised granule (upflow), while Point 2 is located in a non-magnetised downflow. The other two points were chosen at two magnetised sites: Point 3 at a bright magnetic structure and Point 4 in the centre of a dark micropore. The local line profiles of the non-magnetised points are shifted to the blue in Point 1 and to the red in Point 2. For the Fe I line at $617.3 \mathrm{~nm}$, which forms over a wider depth range, the profiles show strong asymmetries caused by velocity gradients along the line of sight. As discussed in Sect. 6.2.1, the 

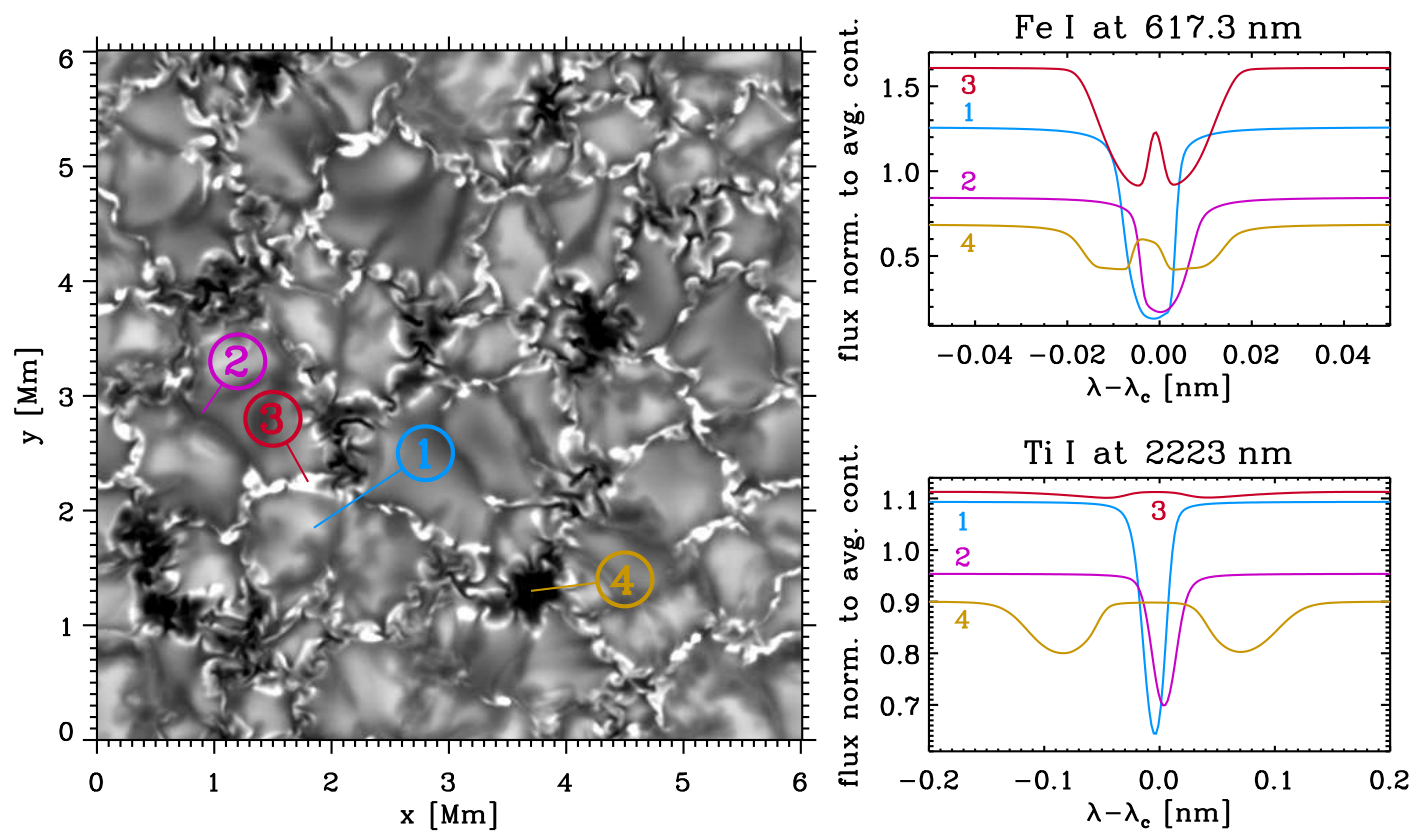

Figure 6.10: Local vertical spectral line profiles for the K0V simulation with $B_{0}=500 \mathrm{G}$. Left panel: intensity image with four positions marked for which local spectra are shown in the right panels: (1) a non-magnetic upflow, (2) a non-magnetic downflow, (3) a bright magnetic structure, (4) a dark magnetic structure. Right panels: local profiles of the Fe I line at $617.3 \mathrm{~nm}($ top $)$ and the Ti I line at $2223 \mathrm{~nm}$ (bottom) at $(\mu=1)$; the four different colours correspond to the four points marked in the left panel.

wings, which originate near the optical surface, have a stronger shift (away from the rest wavelength of the line) than the cores, which are formed in higher layers where the vertical flow speeds are considerably lower. Both lines are subject to the Zeeman effect and are split into two Zeeman components in the magnetic points considered (Point 3 and 4). The excitation potential of the Ti I line much smaller than that of the iron line. Hence, the lower level of the transition becomes depopulated at lower temperatures for the $\mathrm{Ti}$ line as for the Fe line. Moreover, titanium is ionised at a lower temperature than iron. As a result, the $\mathrm{Ti}$ line is very sensitive to temperature at the photospheric temperatures typically prevailing in the K0V star. Consequently, the flux of the (strongly split) line is much higher in Point 4 than it is in Point 3 in spite of the higher continuum flux level in Point 3. The less temperature-sensitive iron line has a comparable equivalent width in Points 3 and 4, but as the continuum flux is more than twice as large at Point 3, the line flux is also higher in this point than in the dark magnetic structure (Point 4). The two Zeeman components are asymmetric in both spectral lines (but rather symmetric with respect to the central wavelength of the line). This can be explained by the gradient of the magnetic field along the line of sight. As the tube-like magnetic structures fan out in the stellar atmospheres, the field strength decreases with height above the surface (cf. Figs. 3.16 and 3.21). Consequently, the wings of the Zeeman components, which form closer to the optical surface than the cores, are more strongly shifted away from the central wavelength of the line than their cores. 

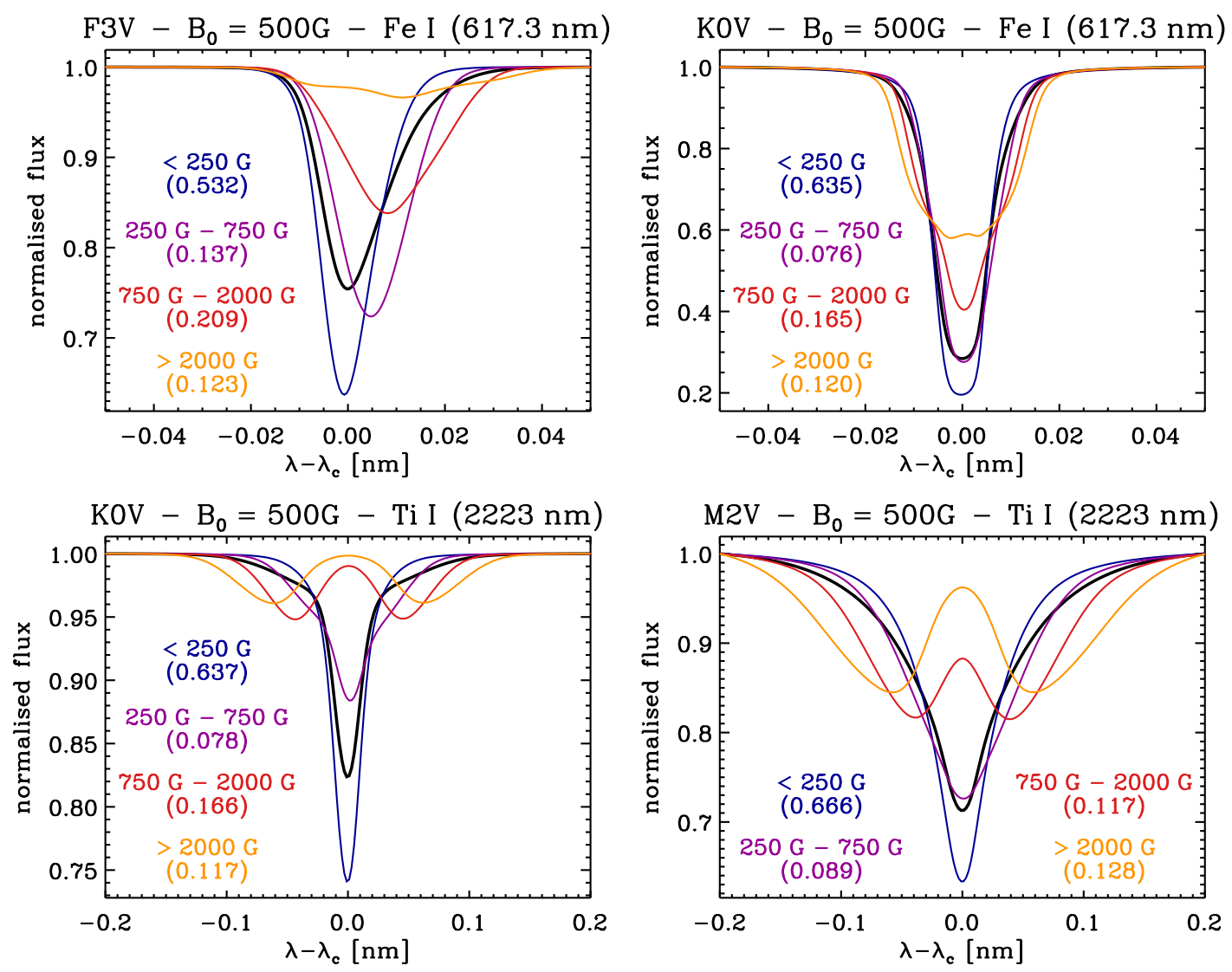

Figure 6.11: Decomposition of four line profiles into differently magnetised components. The black line shows the average vertical $(\mu=1)$ line profile, while the coloured curves show the line averaged over areas where $B\left(\tau_{\mathrm{R}}=1\right)$ is in a certain range (range specified in the plots). Each line is normalised to its own continuum. The numbers in parentheses give the weight of the component in the composite spectrum (see text for further explanation).

Figure 6.11 illustrates for a few combinations of spectral lines and simulations (all with $B_{0}=500 \mathrm{G}$ ), how line profiles originating from regions of different magnetic field strength add up to the average line profile (at $\mu=1$ ). For this analysis, the optical surface was split into four groups of pixels with different field strength: the first group (represented by the dark blue curve in Fig. 6.11) comprises the virtually field-free area $\left(B\left(\tau_{\mathrm{R}}=\right.\right.$ $1)<250 \mathrm{G}$ ), while the second an third groups (purple and red curves, respectively) contain regions with $250 \mathrm{G}<B\left(\tau_{\mathrm{R}}=1\right)<750 \mathrm{G}$ and $\left.750 \mathrm{G}<B\left(\tau_{\mathrm{R}}=1\right)<2000 \mathrm{G}\right)$, respectively. The fourth group (orange) comprises the strongly magnetised area with $\left.B\left(\tau_{\mathrm{R}}=1\right)>2000 \mathrm{G}\right)$. The choice of $B\left(\tau_{\mathrm{R}}=1\right)$ rather than, e.g. $B\left(\tau_{\mathrm{R}}\right)=0.01$ does not strongly influence the result presented in this figure. The average profiles of the four groups are normalised to their respective continua. The product of relative continuum flux level and area fraction of the group yields the weight of the group profile in the average profile. The weights for the four groups are specified in the figure (numbers in parentheses). While all profiles were calculated from simulations with $B_{0}=500 \mathrm{G}$, the impact of the magnetic field on the line profiles is very different in quantity and quality for the 


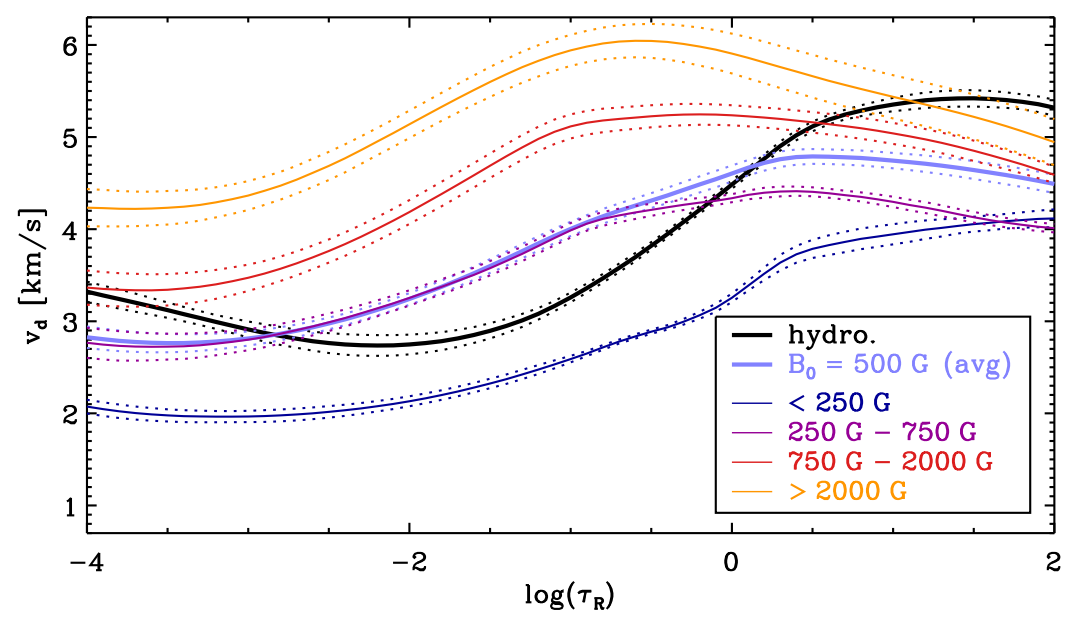

Figure 6.12: Average downflow speed in non-magnetic (hydro.) and $500 \mathrm{G}$ runs of the F3V simulations (thick curves). The thin curves represent the run of the average downflow speed decomposed into the four groups of pixels different $B\left(\tau_{\mathrm{R}}\right)$ introduced in Fig. 6.11. Dotted lines give the 1- $\sigma$ scatter of the six snapshots analysed.

different spectral types and spectral lines. In the F3V simulation, the flow velocities are of the order of several $\mathrm{km} \mathrm{s}^{-1}$ and as the magnetic field is mainly accumulated in the downflow regions, the average profile of the magnetic regions is strongly redshifted while the non-magnetic profile is slightly blueshifted. The 617.3-nm iron line becomes broader and shallower at higher magnetic field strength. In the regions with $B\left(\tau_{\mathrm{R}}\right)>2000 \mathrm{G}$, which are the hot magnetic structures, the line has a strongly reduced equivalent width and its central depth is only about $3 \%$ of the continuum (compared to $35 \%$ in the non-magnetic group). A similar effect is also seen for the other two lines in this star. The mean profile has a strongly asymmetrical shape: the redshifted, broadened component of the intermediately magnetised regions produces a redshifted line wing, which is much more pronounced in the $500 \mathrm{G}$ run than in the non-magnetic run (cf. Fig. 6.12). In the K0V simulation, the effect of the vertical velocity perturbations becomes small compared to the Zeeman effect for the Fe I line at $617.3 \mathrm{~nm}$. However, the Zeeman splitting at about $2 \mathrm{kG}$ is still comparable to the width of the individual Zeeman components. For the titanium line in the same simulation, the splitting is larger compared to the width of the components, resulting in a strongly split line profile in the intermediate and strong field groups (i.e. at $\left.B\left(\tau_{\mathrm{R}}=1\right)>750 \mathrm{G}\right)$. The equivalent width of the lines from these two groups is considerably smaller than in the unmagnetised group, but as these two groups comprise more than $25 \%$ of the weight in the resulting mean profile, they produce strongly broadened line wings. In the M2V star, the effect is much less obvious: the broadening affects the entire profile and not just the extreme wings, since the line profile of the Zeeman components is wider (owing to pressure broadening).

The fact that the redshifted wing of the $\mathrm{Fe}_{\mathrm{I}}$ line profile at $617.3 \mathrm{~nm}$ is much more pronounced in the $500 \mathrm{G}$ run of the F3V star than in the non-magnetic run is only partly due to the higher filling factor of downflows in the $500 \mathrm{G}$ run. A similar but much smaller effect of a redshifted line wing at the disc centre is also seen in the G2V simulation. As 
shown in Fig. B.21 in Appendix B for the G2V and M2V simulations, the downflow speed averaged on iso- $\tau_{\mathrm{R}}$ surfaces is reduced below the surface but slightly higher in the atmospheres in the $500 \mathrm{G}$ runs compared to the non-magnetic runs (qualitatively similar results are obtained for the other stars). Figure 6.12 shows the average downflow speed in the non-magnetic run (thick black curve) and the $500 \mathrm{G}$ run (thick violet curve) for the F3V star averaged on surfaces of constant optical depth. For the $500 \mathrm{G}$ run, this average is also shown split into four groups analogous to Fig. 6.11. There is a strong relation between average downflow speed and field strength, which only breaks down below the optical surface. The two groups with high field strength have average downflow speed of about 4 and $5 \mathrm{~km} \mathrm{~s}^{-1}$ at $\log \tau_{\mathrm{R}}=-2$, where the non-magnetic run has less than $3 \mathrm{~km} \mathrm{~s}^{-1}$. The area fraction of downflows is $(86 \pm 1) \%$ and $(80 \pm 1) \%$ in these groups at $\log \tau_{\mathrm{R}}=-2$. This explains the redshifted wing of the Fe I line profile $(617.3 \mathrm{~nm})$ in the F3V simulation with $500 \mathrm{G}$, as well as a similar shift in other spectral lines formed at similar optical depth. As discussed in Sect. 3.3.2, longer-lived magnetic flux concentrations cannot sustain downflows. As most of the magnetic flux concentrations on $\mathrm{K}$ and in particular on $\mathrm{M}$ stars are long-lived and show concequently very low vertical flow velocities (cf. Fig. 3.31), the spectral line profiles of $\mathrm{K}$ and $\mathrm{M}$ stars do not show a strongly redshifted line wing.

The results presented in Figs. 6.10 and 6.11 illustrate that different parts of the stellar surface have a different weight in the resulting spectrum. For each single ray this weight is simply the line flux. Figure 6.13 shows the average line flux as function of the local magnetic field strength $B\left(\tau_{\mathrm{R}}=1\right)$ at the optical surface normalised by the average line flux in the first bin $\left(B\left(\tau_{\mathrm{R}}=1\right)<200 \mathrm{G}\right)$ for the same spectral lines for which the profiles are shown in Fig. 6.11. As in Fig. 4.13 (Sect. 4.3.1), bins with less than 1000 points (corresponding to about $0.06 \%$ of all points) are scaled by the logarithm of the number of points, i. e. small symbols represent very small numbers of points rendering these bins as rather insignificant. In the F3V simulation, the magnetic structures are so hot that even the Fe $\mathrm{r}$ line at $617.3 \mathrm{~nm}$ is weakened substantially in places where $B\left(\tau_{\mathrm{R}}=1\right)$ is high. At $2 \mathrm{kG}$, the typical field strength of the magnetic flux concentrations, the line flux is reduced to $20-30 \%$ of the non-magnetic value. For the K0V simulation, the flux of Ti I line decreases with increasing local field strength, while the flux of the Fe I line slightly increases with local field strength. As titanium is ionised at a lower temperature than iron, the Ti line is weakened in the hot magnetic structures of the K0V star, while the iron line is not. The flux of the iron line is even somewhat higher in the magnetic regions, because of two effects: first, the overall flux level is higher in the small magnetic regions (cf. Fig. 6.10), second, the Fe I line is saturated to a high degree in this star. The individual Zeeman components are less saturated and consequently the line flux of the split or broadened line is higher. For this spectral type, the runs with different $B_{0}$ show somewhat different line flux in strongly magnetised regions. This is an effect of the dark, pore-like structures which form only at higher $B_{0}$ (cf. Figs. 3.15, 4.12, and 4.13). For the titanium line, which is very temperature-sensitive in this temperature regime, the line flux is less reduced at higher $B_{0}$, because of the onset of the formation of dark micropores. In contrast, the Fe line flux gets less enhanced at higher $B_{0}$ due to the lower average (continuum) intensity of the (dark) magnetic regions, in spite of the reduction of the saturation by the splitting. In the M-star simulations, the line flux is relatively independent of the local field and of $B_{0}$ for all three lines investigated. The variation is smaller because the temperature and intensity contrasts in the M-star atmospheres are smaller than in the atmospheres of hotter 

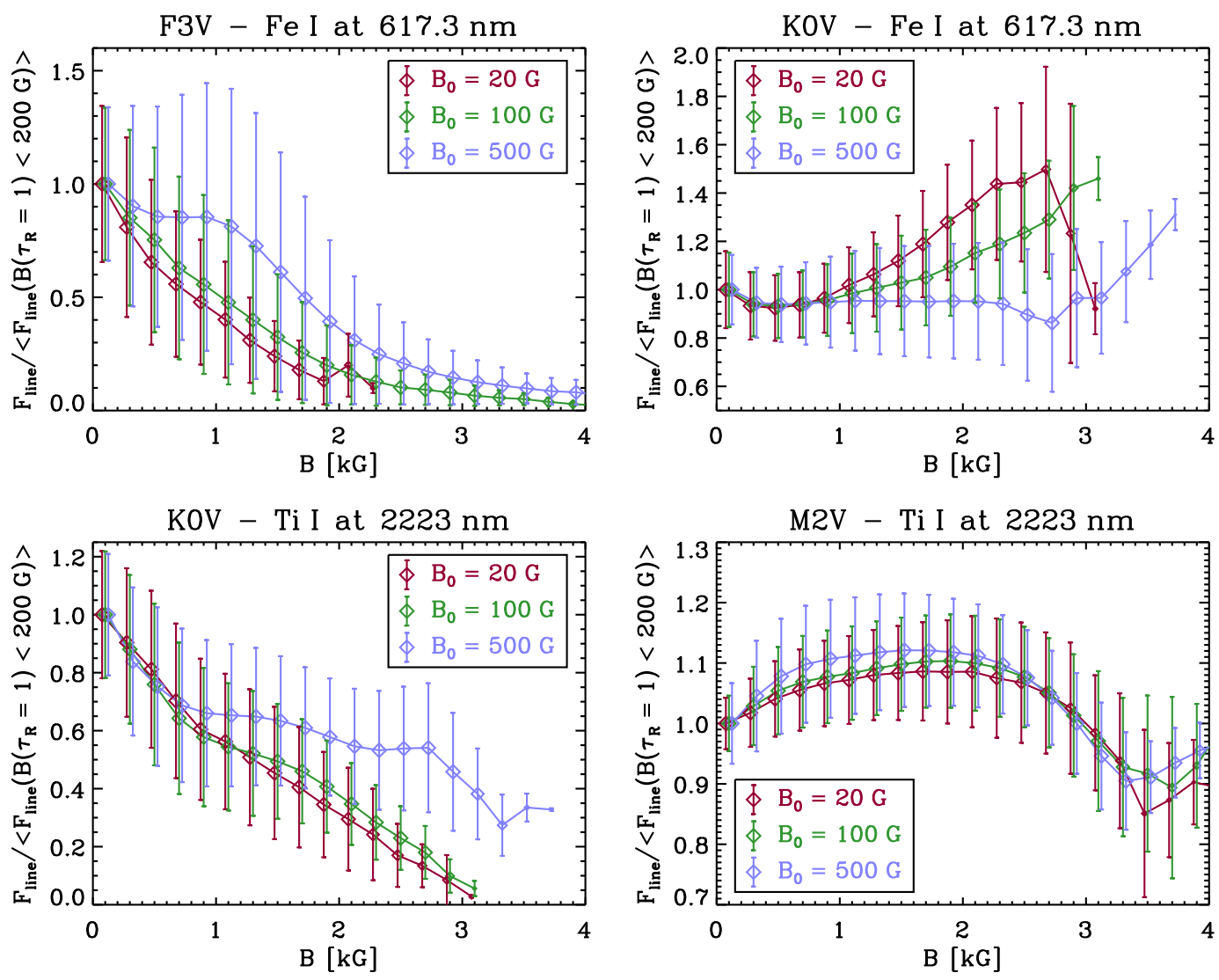

Figure 6.13: Relation between magnetic field strength $B\left(\tau_{\mathrm{R}}=1\right)$ at the optical surface and line flux (cf. Fig. 6.9). The field is binned in steps of $200 \mathrm{G}$, and the line flux is given relative to the line flux in the first field bin $\left(B\left(\tau_{\mathrm{R}}=1\right)<200 \mathrm{G}\right)$. Error bars indicate the $1-\sigma$ scatter in the bins, the slight horizontal shifts between the plot symbols and error bars of different simulations are added for clarity. The size of symbols representing bins with less than 1000 points $(0.06 \%$ of all points) is scaled with the logarithm of the number of points in this bin.

stars.

Figure 6.14 summarises the line flux difference between magnetic and non-magnetic regions for all three spectral lines investigated and all 18 magnetic simulation runs. In this figure, the ratio between the line flux averaged over regions with $B\left(\tau_{\mathrm{R}}=1\right)>1400 \mathrm{G}$ and the line flux averaged over regions with $B\left(\tau_{\mathrm{R}}=1\right)<600 \mathrm{G}$ is plotted versus spectral type. For the three lines investigated here, the line flux produced in magnetic regions is reduced in the F- and G-star simulations. In the M stars, the average line flux does not differ strongly between non-magnetic and magnetic simulations. In the $\mathrm{K}$ stars, the result depends on the spectral line: while the titanium line has a lower flux in the magnetic runs than in the non-magnetic run, the flux of the iron lines are mostly unaffected. The result for the $500 \mathrm{G}$ run differs somewhat from the result for the other two values $B_{0}$, which in turn are very similar. In the F-, G-, and K-star simulations, the formation dark magnetic structures influences the line flux of magnetic regions, and such dark structures form only 


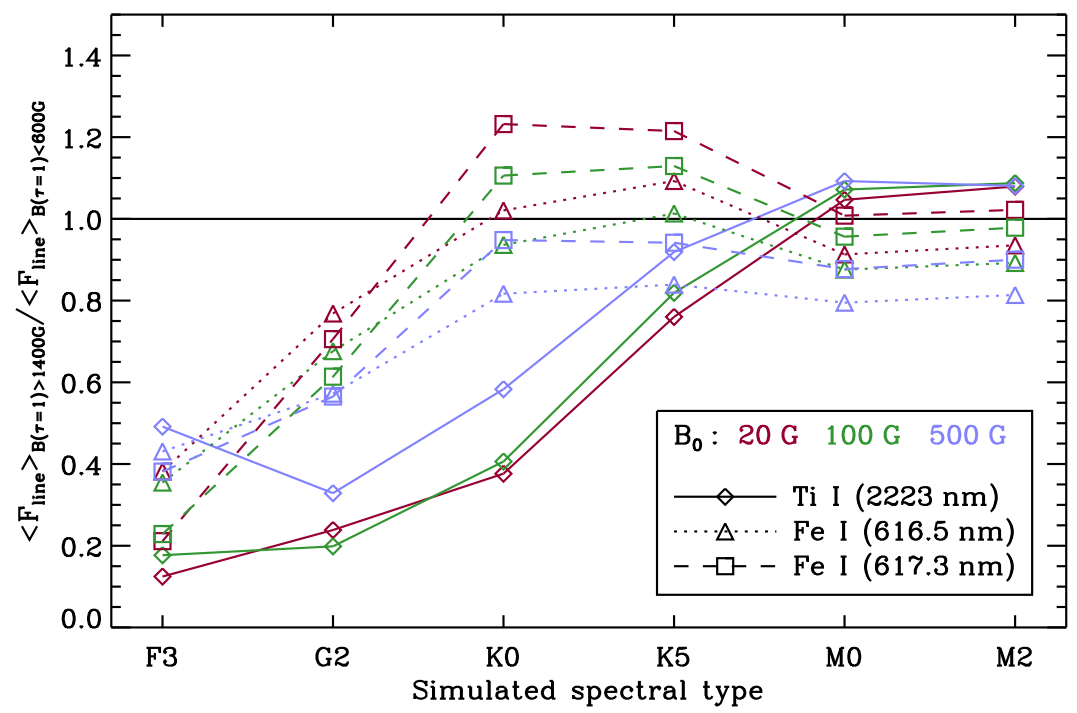

Figure 6.14: Ratio of the line flux $\left\langle F_{\text {line }}\right\rangle_{B\left(\tau_{\mathrm{R}}=1\right)>1400 \mathrm{G}}$, averaged over the parts of the surface where $B\left(\tau_{\mathrm{R}}=1\right)>1400 \mathrm{G}$, to the line flux $\left\langle F_{\text {line }}\right\rangle_{B\left(\tau_{\mathrm{R}}=1\right)<600 \mathrm{G}}$, averaged over the parts of the surface where $B\left(\tau_{\mathrm{R}}=1\right)<600 \mathrm{G}$ (first three bins in Fig. 6.13). Different symbols and linestyles represent the different spectral lines investigated.

in the $500 \mathrm{G}$ runs of these stars.

The results presented in this section show that magnetic and non-magnetic regions do not appear in the spectrum weighted with their area fraction. For some spectral lines, the modified thermodynamics locally reduce the line flux and thus reduce the Zeeman-effect signal of the magnetic field in the composite spectrum. If these lines were used for a determination of the magnetic field without any knowledge of the local thermodynamic changes, the field or filling factor would be strongly underestimated (cf. Sect. 6.3.4).

\subsubsection{Centre-to-limb variation of spectral line profiles}

In the previous section, the analysis was restricted to the spectral line profiles in vertically emerging light (i.e. $\mu=1$ ). In this section, the effect of the magnetic field on the spectral lines profiles is discussed for different values of $\mu$ in terms of the four parameters equivalent width, full width at half maximum, convective Doppler shift of the line core and of the line wings. In Sect. 6.2.2, it was shown that these parameters vary across the stellar discs in the non-magnetic case. The reason for this $\mu$-dependence is the variation of temperature, pressure, density, and line-of-sight velocity along the optical paths of different inclination due to the 3D structure of the surface layers. For the magnetic simulation runs, the magnetic field along the optical path, which acts on some spectral lines by virtue of the Zeeman effect, can likewise statistically vary with $\mu$. An additional impact of the magnetic field on the centre-to-limb variation of spectral line profile shapes can be expected owing to the local modifications of the thermodynamic structure by the field (see Sect. 3.3). As discussed in this section and in Sect. 6.3.4, the effect of the modified thermodynamic structure on the three spectral lines investigated turns out to be more 

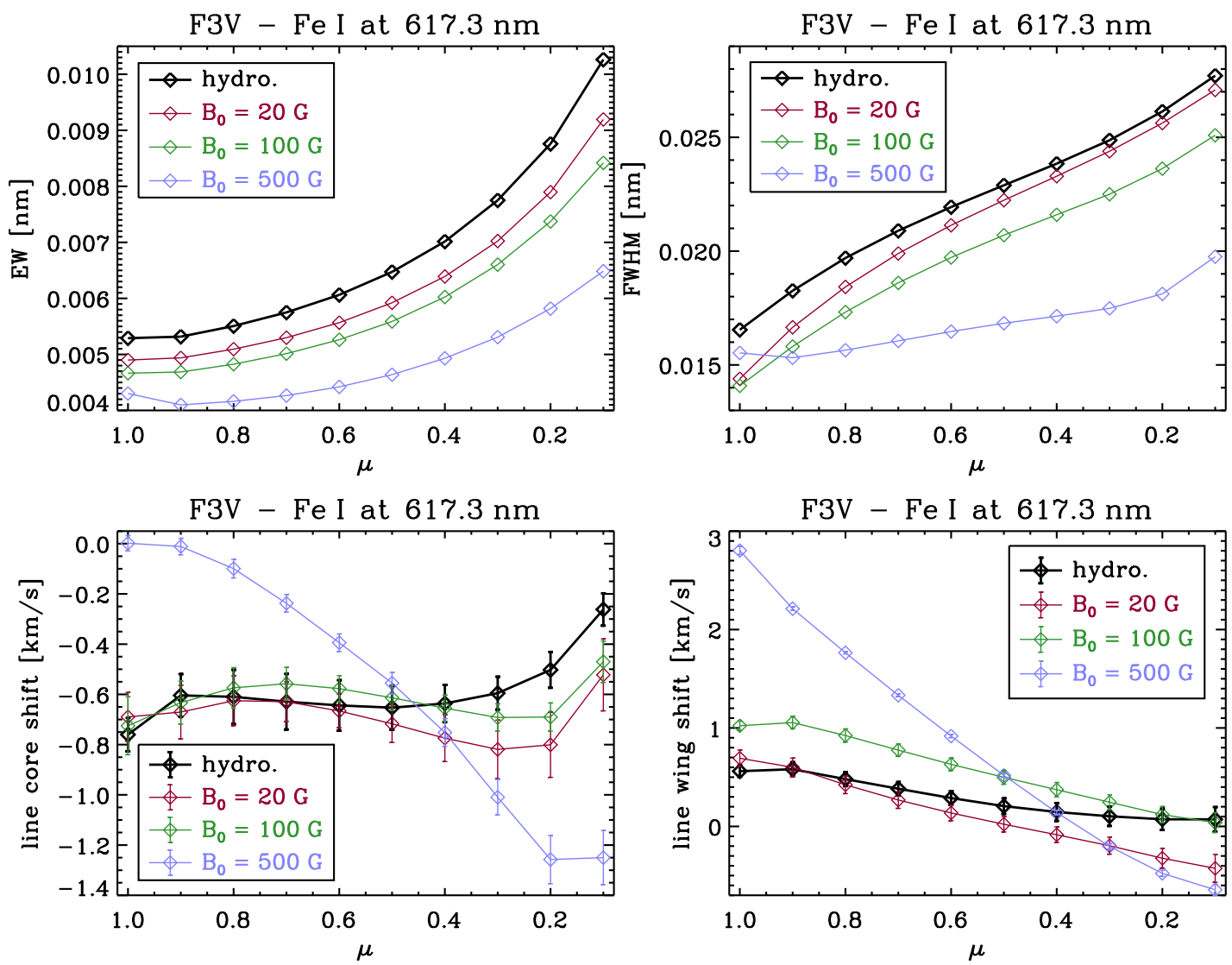

Figure 6.15: Centre-to-limb variation of the Fe I line at $617.3 \mathrm{~nm}$ in the F3V simulations. Shown is the centre-to-limb variation of the equivalent width (top left), the full width at half maximum (top right), the Doppler shift of the line core (positive = redshift; bottom left), and the Doppler shift of the line wings (bottom right). The black curve represents the non-magnetic run (hydro.), the three coloured curves represent the magnetic runs with different values of $B_{0}$.

important than the Zeeman effect in F- and G-type stars for all magnetic field strengths considered here.

Figure 6.15 shows the centre-to-limb variation of the equivalent width (EW), full width at half maximum (FWHM), and the Doppler shifts of the line core and the line wings of the profile of the Fe I line at $617.3 \mathrm{~nm}$ in the F3V simulations. The EW and FWHM increase towards the limb in this star, because the geometrical height of line formation moves upwards where the temperature is lower (less ionisation of Fe I; see Sect. 6.2.2). The general increase in EW and FWHM with decreasing $\mu$ can be observed in all four runs. In addition both parameters are decreasing with increasing $B_{0}$. For the equivalent width, this decrease is due the line weakening (owing mainly to ionisation of $\mathrm{Fe}$, but also to the lower temperature gradient and to the decreased population of the lower level of the atomic transition) in the hot magnetic structures (cf. Fig. 6.13). The line weakening does not affect the FWHM much. Consequently, the reduction of the FWHM of the line near the disc centre is smaller than the reduction of EW. As the horizontal rms 

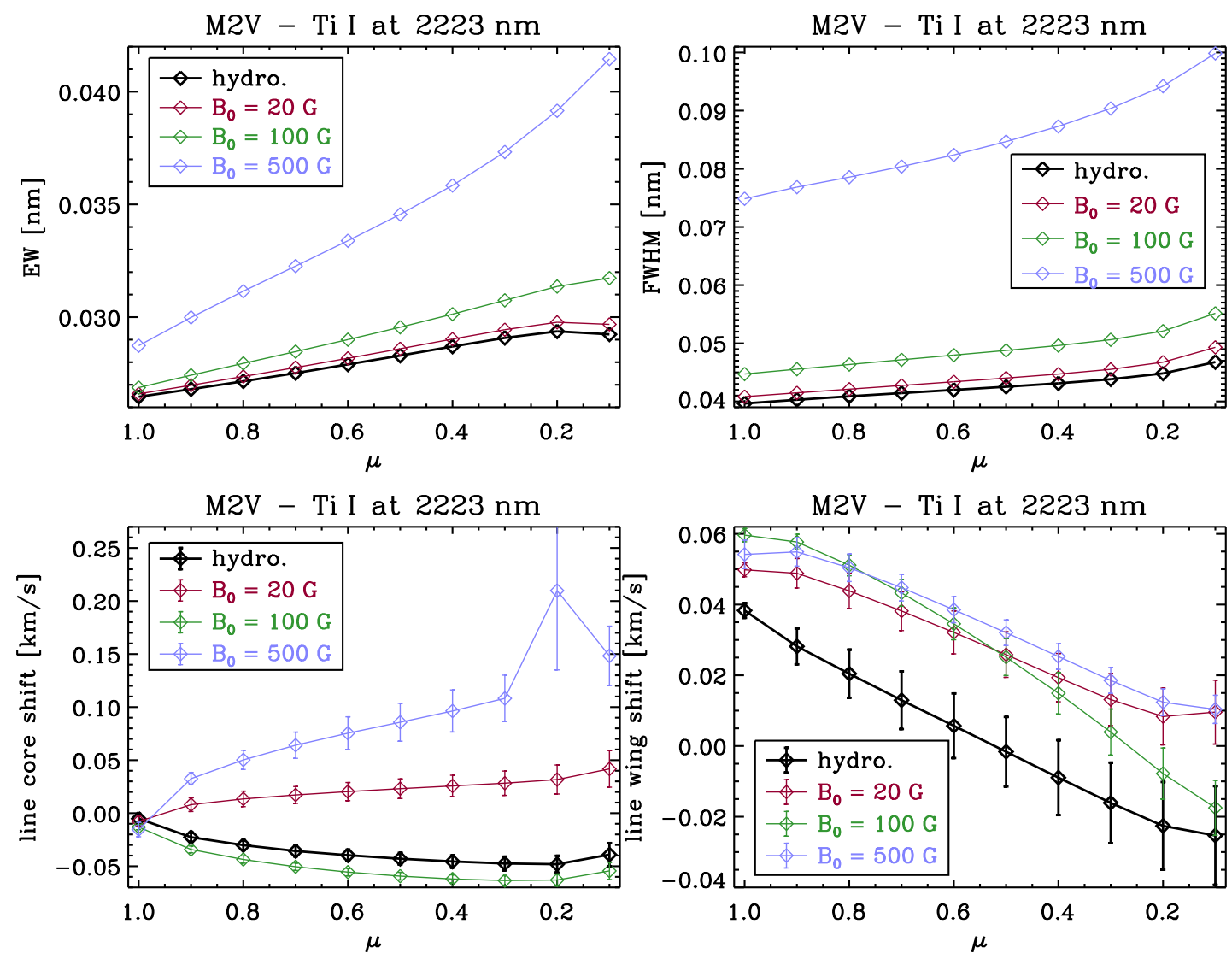

Figure 6.16: Same as Fig. 6.15, but for the Ti I line at $2223 \mathrm{~nm}$ and the M2V simulations.

flow speeds are reduced in the magnetic runs (cf. Fig. 3.28), the "turbulent broadening" of the line is decreased near the limb. This has no strong effect on the EW, but leads to a reduction of the FWHM at low $\mu$.

As pointed out in Sect. 6.3.1 (see discussion of Figs. 6.11 and 6.12), the magnetic field has also an impact on the effective Doppler shift of the line profile. In the non-magnetic run, the $\mathrm{Fe}_{\mathrm{I}}$ line at $617.3 \mathrm{~nm}$ has a blueshifted line core and redshifted line wings at the disc centre and almost unshifted cores and wings near the limb (cf. Fig. 6.3). In the $500 \mathrm{G}$ run of the $\mathrm{F} 3 \mathrm{~V}$ star, the line core is no longer blueshifted at the disc centre, but more strongly blueshifted near the limb, while the wings are much more strongly redshifted (about $3 \mathrm{~km} \mathrm{~s}^{-1}$ ) than in the non-magnetic case (less than $1 \mathrm{~km} \mathrm{~s}^{-1}$ ) at the disc centre but still almost unshifted near the limb. The modified flows in the magnetic flux concentrations and the much more strongly corrugated optical surface are mainly responsible for these changes. Near the disc centre, the deep depressions caused by the magnetic flux concentrations lead to the strong redshift in the line wings (cf. Fig. 6.11), whereas closer to the limb, the flux concentrations are hidden from view (cf. Fig. 5.4) and this shift is consequently not present.

Figure 6.16 shows the centre-to-limb variation of the line profile parameters for the $\mathrm{Ti}$ I line in the M2V star. There are qualitative and quantitative differences to the F3V star discussed above. In contrast to the F3V star, EW and FWHM are increasing with $B_{0}$. For 
the EW, this increase is strongest near the limb, while for the FWHM the relative increase is almost independent of $\mu$. For the FWHM, this can be attributed to the Zeeman effect, which substantially broadens this line in this star (cf. Fig. 6.11). The Zeeman effect also has an influence on the EW: although the magnetic structures have reduced intensity, the flux of this line is slightly increased in these regions mostly due to the reduced degree of saturation in the individual Zeeman components (cf. Fig. 6.13). The increase is stronger near the limb, probably due to the side walls of the magnetic structures, where the line forms in the cool region above and in front of the magnetic structure before a bright continuum background of the side wall.

The variation of the Doppler shifts of line core and wings with $B_{0}$ is very small for this star. There is a small tendency for redshifted lines at higher $B_{0}$. This tendency might be caused by the increased line flux within the magnetic structures: although large parts of these structures are virtually at rest, the smaller ones are associated with downflows, which then contribute substantially to the spectrum due to their high area fraction and the increased line flux. The difference between the Doppler shifts of runs with different $B_{0}$ are, however, not very significant and the magnitude of the supposed effect does not scale well with $B_{0}$. The observed impact of the magnetic field on the line shifts could be coincidental in this star.

In Figure 6.17, the difference of the EW and FWHM of the three spectral lines between the $500 \mathrm{G}$ runs and the respective non-magnetic runs are shown. As outlined in the discussion of Figs. 6.15 and 6.16, this difference can be attributed mainly to three effects: Zeeman effect, line weakening, and modified flows. The Zeeman effect leads to an enhanced FWHM. To a lesser extend, it also enhances the EW (due to the reduction of line saturation by the Zeeman splitting into several components). As the relative strength of the $\sigma$ components of the line to the $\pi$ component depends on the field direction, the impact of the Zeeman effect should decrease with $\mu$ as the angle between magnetic field and line of sight becomes larger. The magnitude of the Zeeman splitting or broadening also depends on the line parameters $\left(g_{\mathrm{eff}}, \lambda\right)$. In contrast, the line weakening (due to ionisation) entails a reduced EW and, to a lesser extend, also a reduced FWHM (if the line weakening is correlated with the velocity field). The line weakening should depend mildly on $\mu$, but not on $\lambda$ and $g_{\text {eff }}$. The modified flows can also considerably change the centre-to-limb variation of the FWHM and the EW as discussed in detail for the F3V star above (see discussion of Fig. 6.15). The impact of the modified flows is strongly depending on $\mu$ and only weakly depending on the line parameters. Figure 6.17 thus illustrates the balance between these three effects. In the F3V star, line weakening and modified flows dominate over the Zeeman effect in all three lines (reduced, $\mu$-dependent EW and FWHM in the $500 \mathrm{G}$ run compared to the non-magnetic run). In the G- and K-star simulations, the effect of the line weakening is smaller. In the $\mathrm{K}$ stars it only plays a role for the titanium line (lower ionisation temperature). In the $\mathrm{M}$ stars, most of the impact of the magnetic field on the EW can be attributed to the Zeeman effect. Consequently, it is strongest in the infrared titanium line because the Zeeman broadening depends quadratically on $\lambda$ whereas other broadening mechanisms scale linearly with $\lambda$. For the two iron lines, the Zeeman broadening is much stronger for the $617.3 \mathrm{~nm}$ line because of its effective Landé factor of $g_{\text {eff }}=2.5$ compared to $g_{\text {eff }}=0.69$ of the $616.5 \mathrm{~nm}$ line.

Figures 6.18 and 6.19 give the differences of the Doppler shifts of line cores and line wings, respectively, between the $500 \mathrm{G}$ runs and the respective non-magnetic runs. 

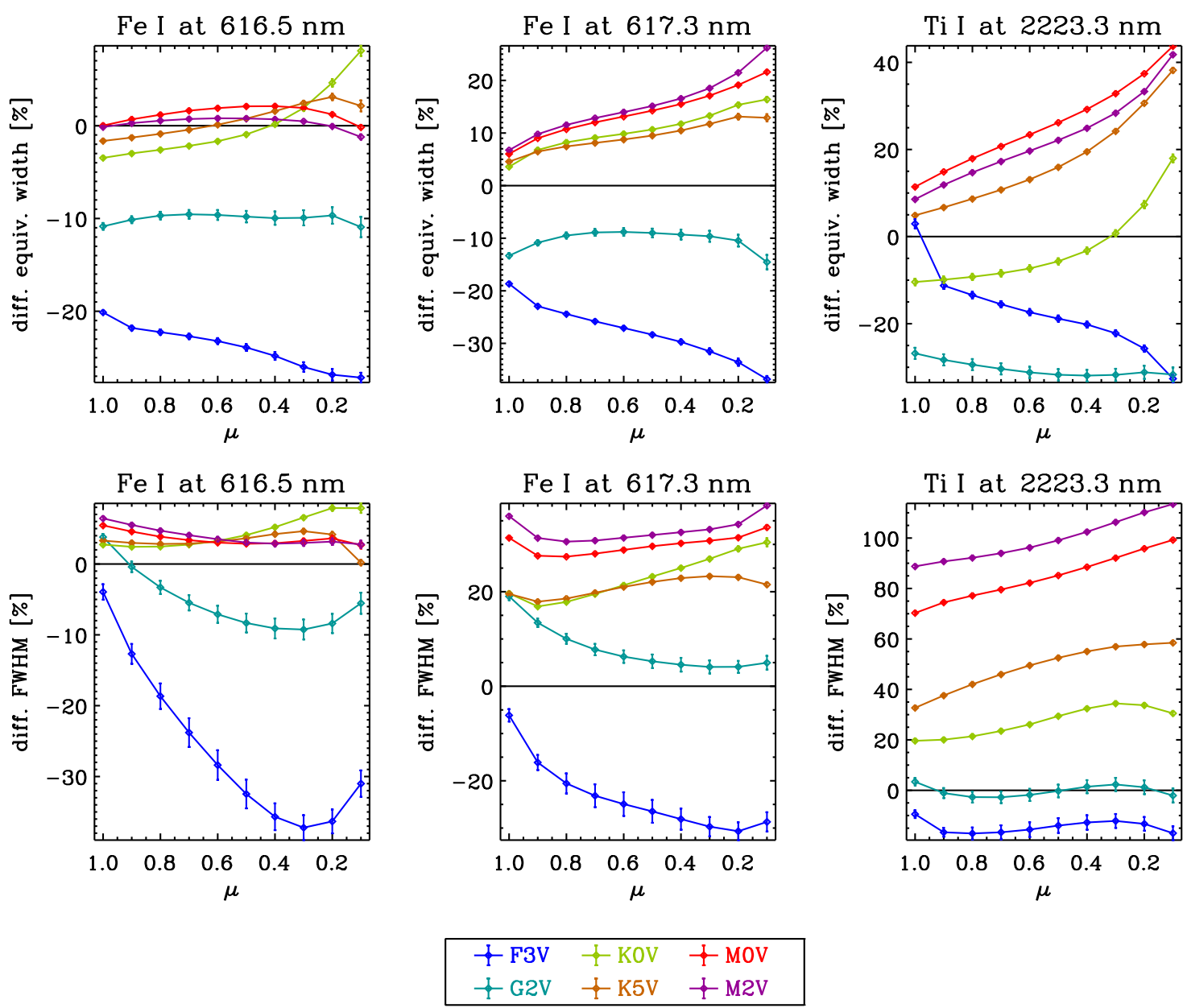

Figure 6.17: Difference of equivalent width (upper panels) and FWHM (lower panels) between simulations with $B_{0}=500 \mathrm{G}$ and the respective non-magnetic simulations for the three spectral lines investigated as function of $\mu$.

The impact of the magnetic field on the Doppler shift of the line cores and wings is qualitatively similar for all spectral lines, in spite of their slightly different formation heights. For the F3V and G2V stars, core and wings are relatively shifted to the red at disc centre and to the blue near the limb. The reason of the relative redshift at the disc centre is the strongly enhanced downflow speed in the magnetic flux concentrations (cf. discussion of Fig. 6.15), while the relative blueshift near the limb is caused by the strong corrugation of the optical surface: downflows are mosly hidden behind granules and have a low lineof-sight velocity component, whereas the granule tops approaching the observer are less obscured by the receeding flows in front of them than without magnetic field. For the other spectral types, the impact is small, with a small tendency towards a relative redshift in the $\mathrm{K} 5 \mathrm{~V}$ and $\mathrm{M} 2 \mathrm{~V}$ simulations, which might, however, be coincidental because its significance is low (see discussion of Fig. 6.16). 

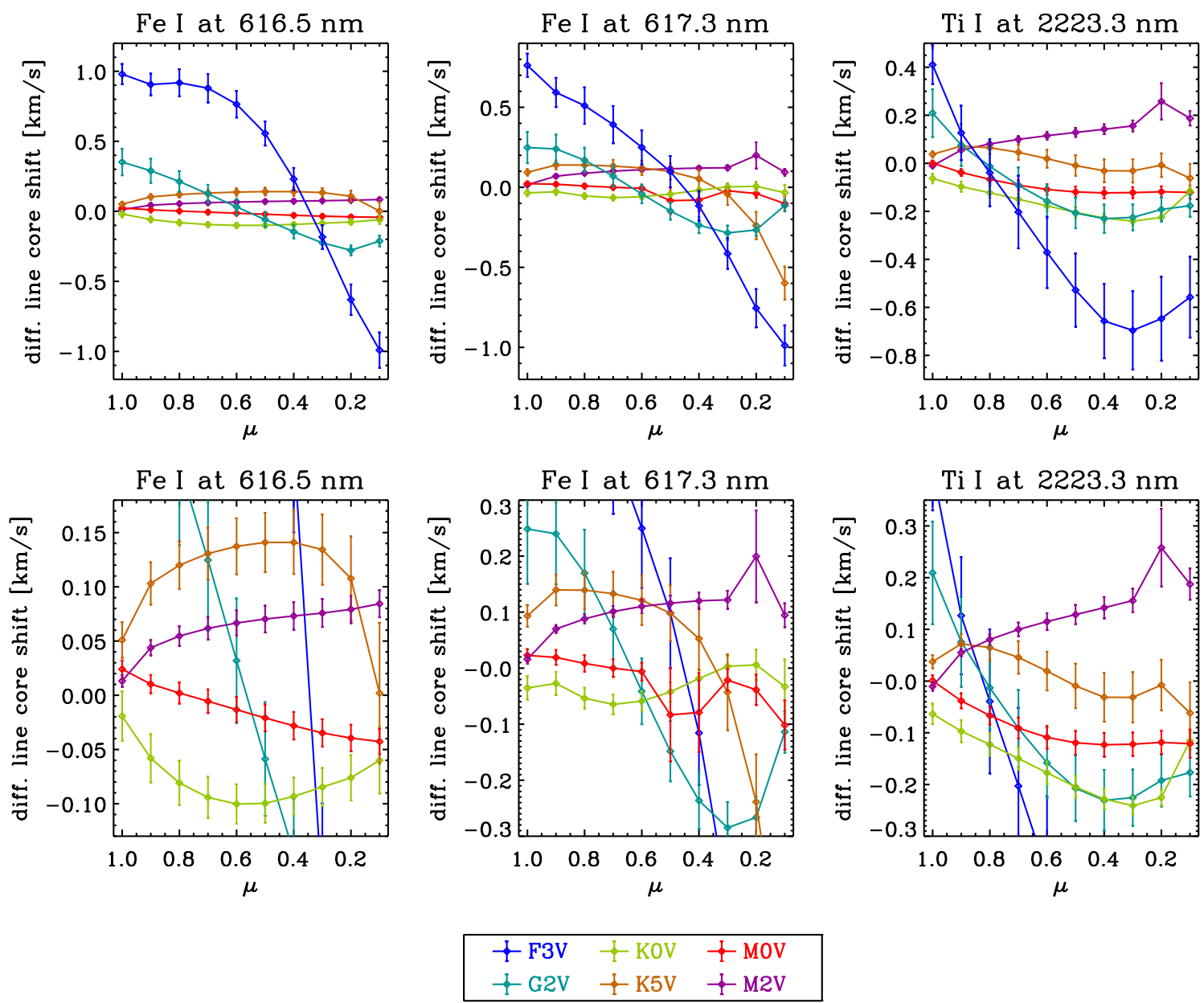

Figure 6.18: Difference of the line core Doppler shift between simulations with $B_{0}=$ $500 \mathrm{G}$ and the respective non-magnetic simulations as function of $\mu$ for the three spectral lines considered. The lower panels are a zoom-in on the curves for the cooler stars.

\subsubsection{Disc-integrated spectra}

For the spectral lines presented in this section, the numerical disc-integration method presented in Sect. 6.1 was applied assuming a homogeneous large-scale distribution of the magnetic field over the visible stellar surface. The consequences of this somewhat unrealistic assumption are discussed in Sect. 7.2.

Figure 6.20 shows disc-integrated line profiles for a few combinations of spectral lines and spectral types. The dashed curves represent the profiles without rotation. For the solid curves the profiles were broadened with the solar differential rotation $(\alpha=0.2)$ at a rotation velocity of $v_{\text {rot }} \sin i=7.5 \mathrm{~km} \mathrm{~s}^{-1}$ and an inclination of $i=60^{\circ}$. This is a reasonable rotation rate for moderately active stars of spectral type $\mathrm{F}$ and $\mathrm{G}$, as well as for relatively young and active $\mathrm{K}$ and $\mathrm{M}$ dwarfs.

In the F3V simulation, both iron lines are shallower and narrower in the $500 \mathrm{G}$ runs than in the non-magnetic runs. Moreover, the lines are considerably shifted to the red and their red wings become more prominent. As already discussed in Sects. 6.3.1 and 6.3.2, 

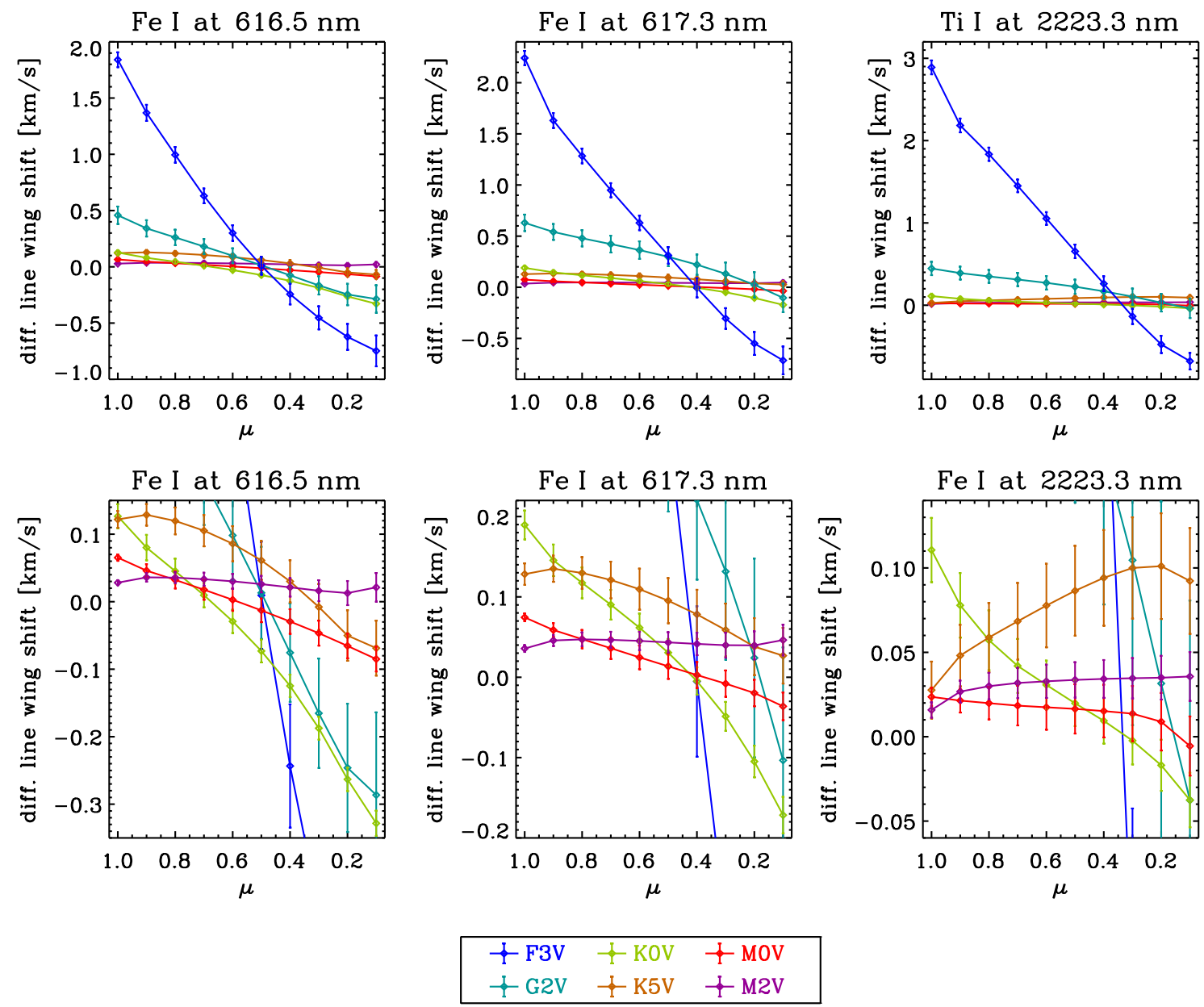

Figure 6.19: Same as Fig. 6.18 but for the difference of the line wing Doppler shift.

the line weakening ${ }^{3}$ and the modified flows play a more important role in this star than the Zeeman effect (cf. Figs. 6.11 and 6.15). The modifications of both iron lines by the magnetic field look thus very similar although the sensitivity to the Zeeman effect is rather different. In the cooler K0V star, the line weakening is unimportant for the iron lines, but still important for the titanium line (cf. Fig. 6.10). Although the Zeeman broadening is stronger in the infrared titanium line than in the $617.3 \mathrm{~nm}$ iron line, the EW of the TiI line is slightly reduced in the magnetic run and its FWHM similar to the non-magnetic run without rotation. The Zeeman effect, however, shifts some line flux to the extreme wings (cf. Fig. 6.11). For the Fe I line at $617.3 \mathrm{~nm}$, the line weakening does not play a role. Therefore, EW and FWHM are larger in the line profiles of the $500 \mathrm{G}$ run at any rotation rate. In the M2V simulations, there is no line weakening, thus EW and FWHM are higher in the $500 \mathrm{G}$ runs than in the non-magnetic runs for all lines owing to the Zeeman effect. Owing to the larger wavelength and high $g_{\text {eff }}$, the titanium line shows the strongest Zeeman broadening in this star.

\footnotetext{
${ }^{3}$ The line weakening is less obvious in the disc-integrated profile without rotation because its effect is mainly on the EW and not so much on the line depth.
} 

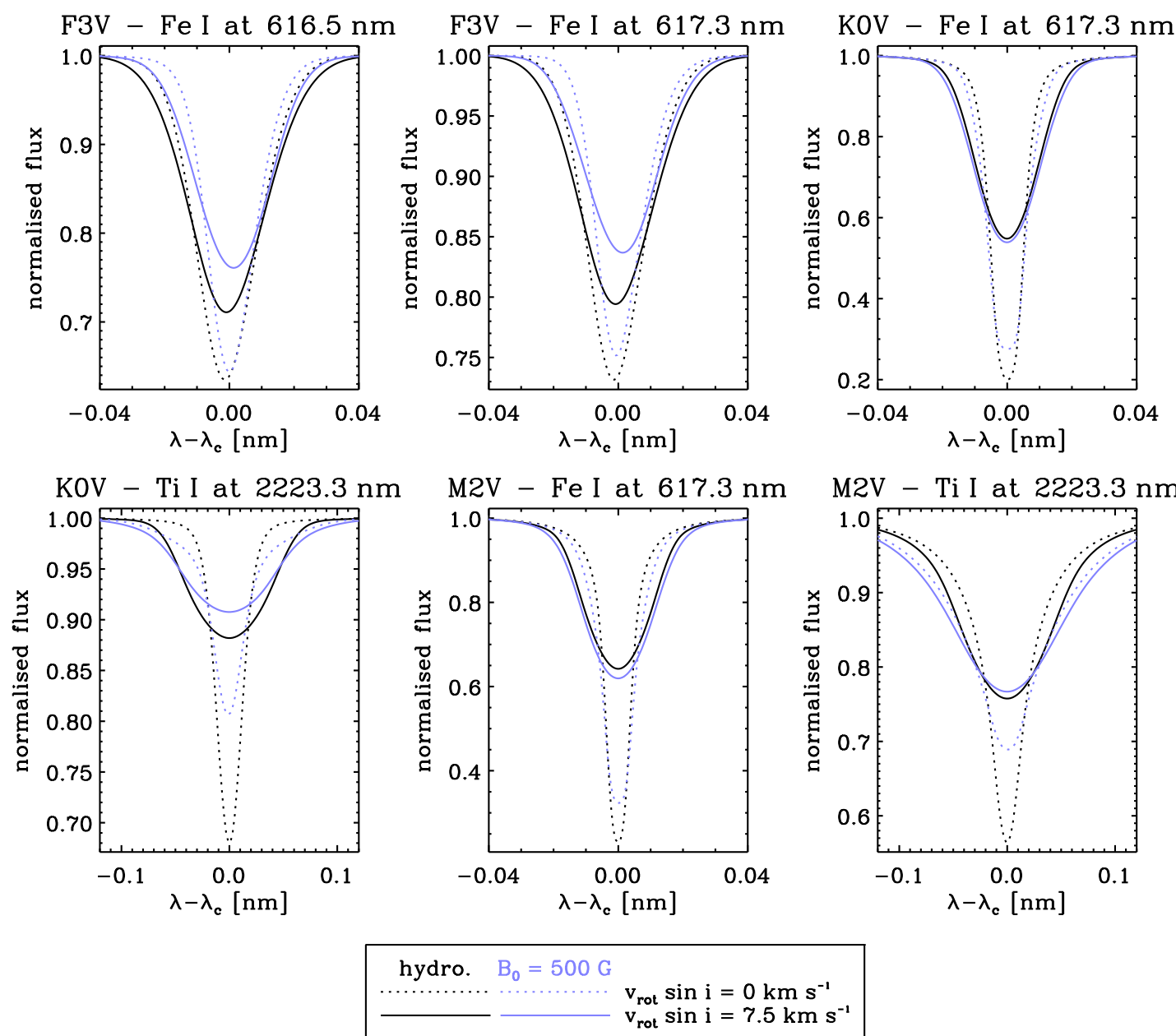

Figure 6.20: Disc-integrated line profiles of six combinations of spectral lines and spectral types. In all panels the black curves correspond to the non-magnetic run and the violet curves correspond to the $500 \mathrm{G}$ runs. The dashed curves represent the disc-integrated line profiles without rotation, while the solid curves include broadening by a differential rotation (solar, $\alpha=0.2$ ) at $v_{\text {rot }} \sin i=7.5 \mathrm{~km} \mathrm{~s}^{-1}$ seen at an inclination of $60^{\circ}$.

Figure 6.21 presents the values of the EW and FWHM of all disc-integrated line profiles without stellar rotation. The line weakening shows up very clearly in the EW of the hotter end of the model sequence, while the broadening due to the Zeeman effect is best visible in the FWHM of the cooler end of the model sequence. The Zeeman broadening shows the strongest effect in the titanium line, as expected, while it is almost not present in the Fe I line at $616.5 \mathrm{~nm}$ with its low effective Landé factor of $g_{\text {eff }}=0.69$. The virtual absence of the Zeeman effect in this line renders the whole extent of the line weakening visible, while it is partly compensated by the Zeeman effect in the other two spectral lines. It is important to note that the Zeeman broadening appears to be roughly proportional to $B_{0}$ (in both EW and FWHM), which is well visible for the M stars, while the line weakening increases less than linearly with $B_{0}$.

As shown in terms of bisectors in Figs. 6.5 - 6.7 in Sect. 6.2.3, stellar rotation can distort already asymmetric line profiles and thus have an impact on the line shape, in 

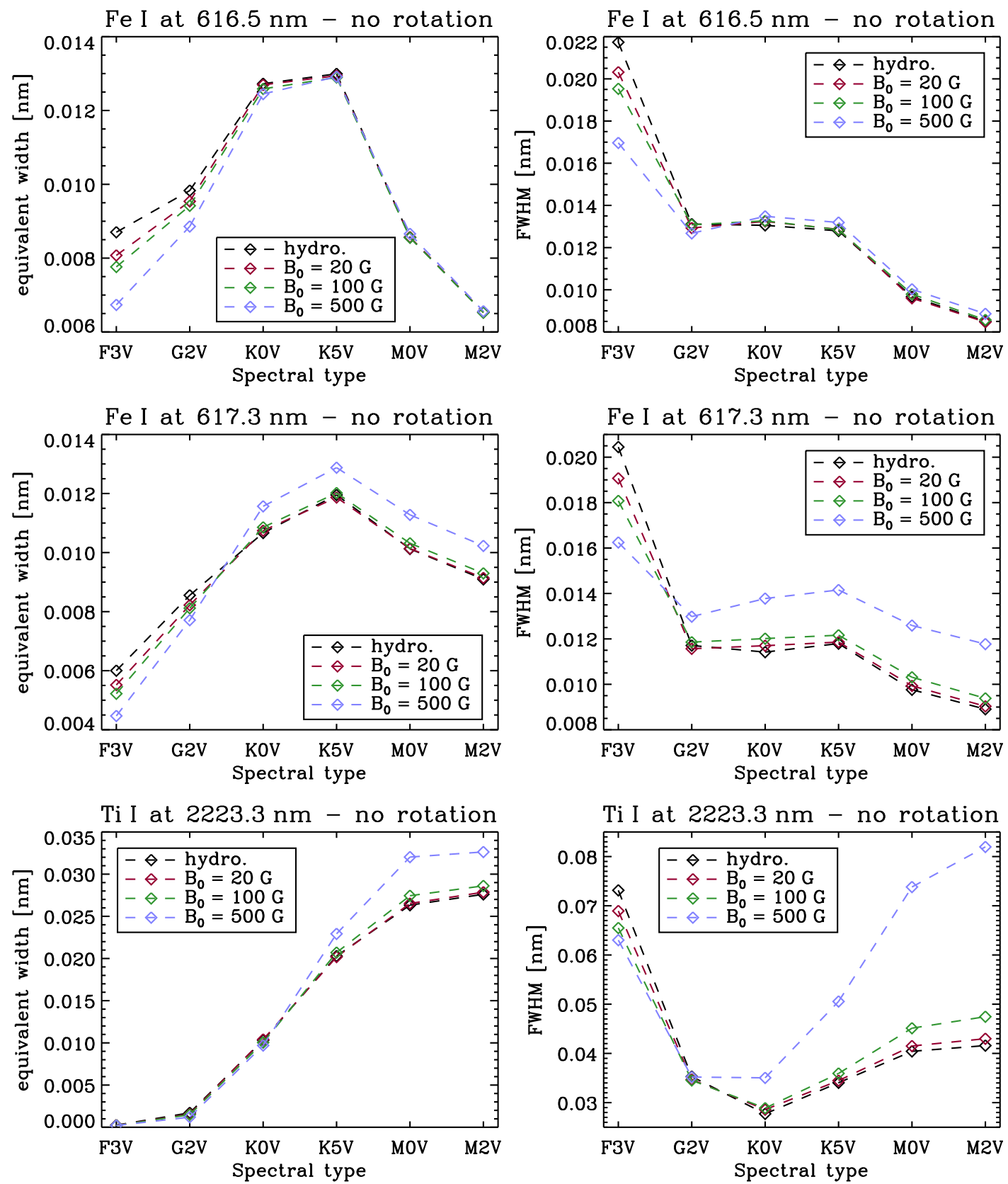

Figure 6.21: Equivalent width (left panels) and full width at half maximum (FWHM, right panels) of the profiles of the three investigated lines for all 24 simulation runs. 

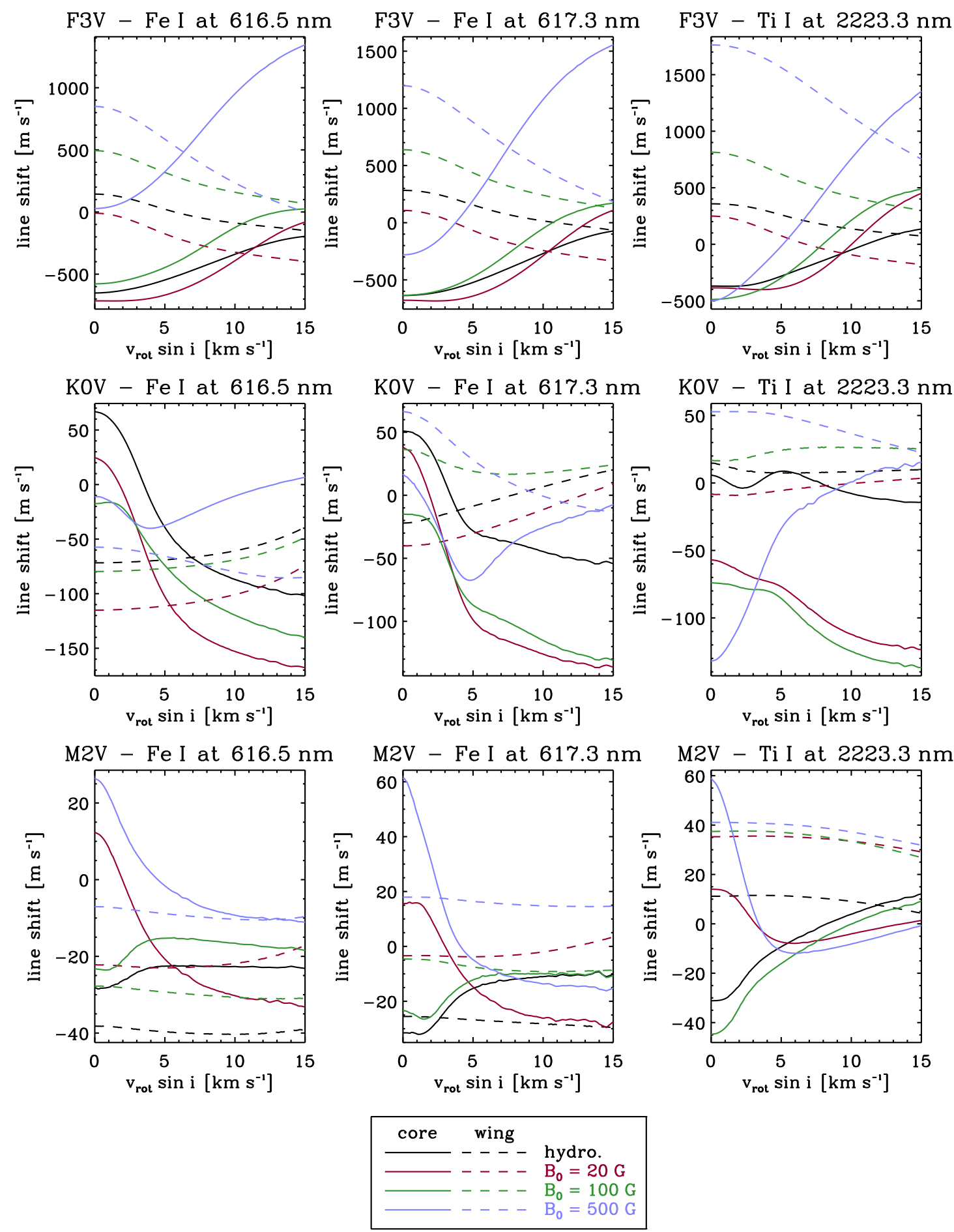

Figure 6.22: Doppler shift of the line profile cores (solid curves) and wings (dashed curves) of all three spectral lines investigated in the $\mathrm{F} 3 \mathrm{~V}, \mathrm{~K} 0 \mathrm{~V}$, and $\mathrm{M} 2 \mathrm{~V}$ simulations as function of $v_{\text {rot }} \sin i$ (for the same plot for the $\mathrm{G} 2 \mathrm{~V}, \mathrm{~K} 5 \mathrm{~V}$, and M0V simulations see Fig. B.26). In all cases, $i=60^{\circ}$ and $\alpha=0.2$ was assumed. 
particular on the effective Doppler shift of the line core and its wings. Figure 6.22 illustrates the impact of stellar rotation on the effective Doppler shift of the lines as function of the rotational velocity. In Sect. 6.3.2 it was illustrated that the Doppler shifts of the lines are strongly affected by the magnetic field near the disc centre in the F- and Gstar simulations (cf. Figs. 6.18 and 6.19). As expected, the highly redshifted line wings of the disc-centre spectrum also have an effect on the disc-integrated spectrum. For the $616.5 \mathrm{~nm}$ Fe I line in the F3V simulation for instance, line wings and core are shifted by approximately 800 and $600 \mathrm{~m} \mathrm{~s}^{-1}$, respectively, to the red in the $500 \mathrm{G}$ run compared to the non-magnetic run. As Fig. 6.22 illustrates, simulations with different $B_{0}$ have different effective Doppler shifts of the lines and, more importantly, a different dependence on $v_{\text {rot }}$. If the simulated stars had large-scale structures in the magnetic field (analogous to active and quiet region on the Sun), the Doppler shift would become time dependent in a non-trivial fashion (also see Sect. 7.2). Reiners et al. (2013) proposed an effect of the Zeeman effect on radial velocity signals by a combination of the Doppler and Zeeman effects similar to the Rossiter-McLaughlin effect (Rossiter 1924, McLaughlin 1924). Fig. 6.22 shows, that the effect of the magnetic field is even more pronounced and probably more complicated than that and not exclusively caused by the Zeeman effect but also by the modified convective flow patterns.

\subsubsection{Zeeman effect vs. thermodynamic effects}

The sensitivity to the Zeeman effect of the three spectral lines investigated is considerably different. The Ti I line and the Fe I line at $617.3 \mathrm{~nm}$ were chosen for their large effective Landé factors (2.5 and 1.66, respectively) and the resulting strong Zeeman splitting, while the $\mathrm{Fe}_{\mathrm{I}}$ line at $616.5 \mathrm{~nm}$ has a lower effective Landé factor of only 0.69 . In the previous section, the impact of the magnetic field on disc-integrated line profiles was analysed. In particular for the two hottest models (F3V and G2V), the effect of the magnetic field is not limited to broadening due to the Zeeman effect, but rather a complicated consequence of the modified thermodynamical structure. In this section, the relative importance of the Zeeman effect will be analysed more quantitatively.

To disentangle the effects of the modified thermodynamical structure (here including the modified flows) and the Zeeman splitting, the spectral line synthesis for the $500 \mathrm{G}$ runs was repeated with the Zeeman effect artificially turned off: the thermodynamical structures were exactly identical to the ones of the $500 \mathrm{G}$ runs, but the magnetic field was neglected for these new line calculations. Figure 6.23 shows two examples of the resulting disc-integrated profiles (Fe I line at $617.3 \mathrm{~nm}$ for the G2V star at two different rotation rates). The grey curves in these plots correspond to the line profiles of the nonmagnetic simulation and the blue curves to the line profiles of the $500 \mathrm{G}$ simulation. The red curve is the line profile of the 500 G-run atmosphere without Zeeman effect (labelled $500 \mathrm{G} / 0 \mathrm{G})$. The difference between the grey and the red curves are thus only due to the local modifications in the atmospheric structure (indirect effect of the magnetic field), whereas the difference between the blue and the red curves are exclusively caused by the Zeeman effect (direct effect of the magnetic field). Without rotation, direct and indirect effects of the magnetic field on the line profile are similar in magnitude for this star and spectral line. At a rotation speed of $v_{\text {rot }} \sin i=7.5 \mathrm{~km} \mathrm{~s}^{-1}$, however, the indirect effects of the magnetic field are much stronger than the direct ones as the line is strongly broadened 

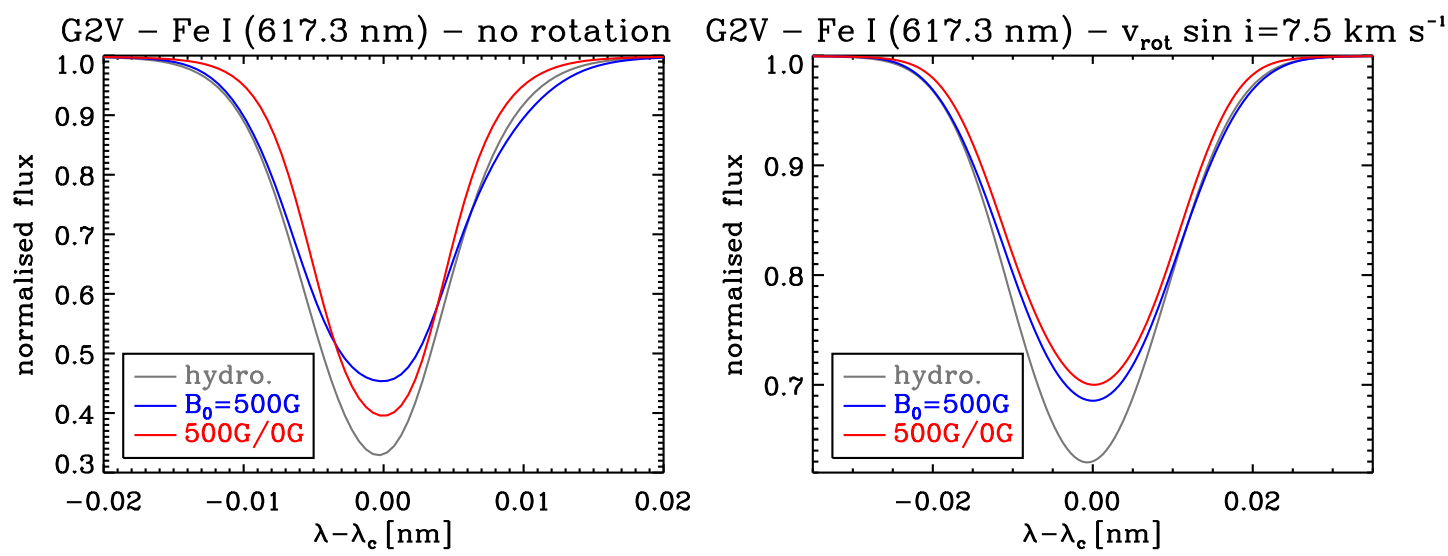

Figure 6.23: Disc-integrated profiles of the Fe I line at $617.3 \mathrm{~nm}$ without rotation (left) and with $v_{\text {rot }} \sin i=7.5 \mathrm{~km} \mathrm{~s}^{-1}$ (differential, $\alpha=0.2, i=60^{\circ}$; right) for G2V simulations. The grey line represents the profile of the non-magnetic run (hydro.), the blue curve is the profile of the $500 \mathrm{G}$ run, the red curve is calculated using the thermodynamic structure of the $500 \mathrm{G}$ run but assuming $B \equiv 0 \mathrm{G}$ for the line calculation (see text for further explanation). Note the different scales of ordinate and abscissa.

by rotation so that the much smaller broadening by the Zeeman effect is largely concealed.

As one possible way of quantifying the differences between the different line profiles shown in Fig. 6.23, the integral of the unsigned differences in the line profiles (with normalised continuum flux) was calculated. The resulting integrated differences are shown for the $\mathrm{Fe}_{\mathrm{I}}$ line at $617.3 \mathrm{~nm}$ and the Ti I line at $2223.3 \mathrm{~nm}$ in Fig. 6.24 for all spectral types. The blue squares in this Figure represent the integrated differences between the 500 G-run atmosphere with and without Zeeman effect (blue and red curves in Fig. 6.23). The red diamonds show the integrated difference between the non-magnetic run and the $500 \mathrm{G}$ run without Zeeman effect (red and grey curves in Fig. 6.23). The blue squares thus represent the impact of the Zeeman effect and the red triangles the impact of the modified atmospheric structure. All values are normalised by the difference between the non-magnetic and the $500 \mathrm{G}$ run with Zeeman effect (difference between blue and grey curves in Fig. 6.23). The sum of both values for the same star is usually above $100 \%$ because unsigned differences are used and the two effects often cause deviations with opposite sign. For example, for the profiles shown in the right panel of Fig. 6.23, the modified thermodynamical structure causes the difference between grey and red profile of the Fe r line. This difference is about 1.7 times as large as the difference between the blue and grey curves, which corresponds to the actually observable difference between the profiles calculated from the non-magnetic and $500 \mathrm{G}$ runs. The Zeeman effect causes the difference between red and blue curve, which is about $70 \%$ of the magnitude of the observed effect. In this case, the effects have opposite signs at all wavelengths, and partly cancel each other.

According to the integrated differences, the modification of the thermodynamic structure can only be neglected in the M stars, where their impact on the line profiles is less than $10 \%$ of the total effect of the magnetic field. In all other stars, the effect of the modified thermodynamical structure is at least comparable to the Zeeman effect for these two 

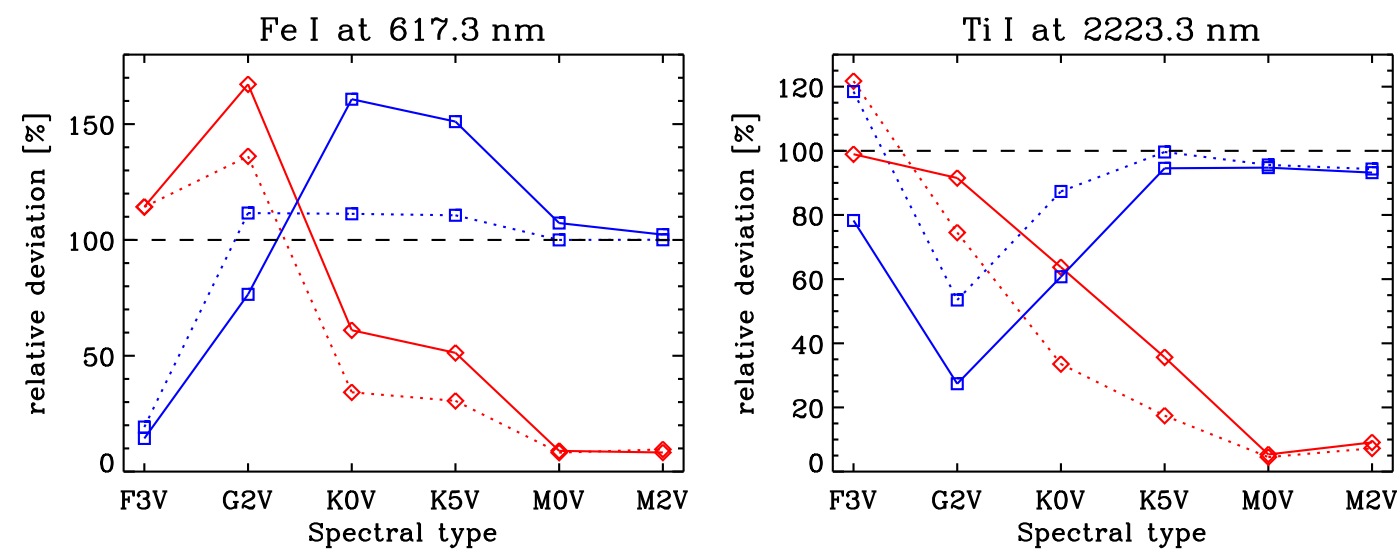

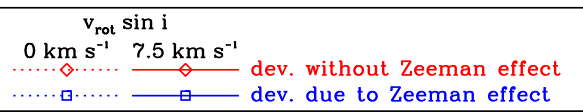

Figure 6.24: Integrated unsigned deviation between differently obtained line profiles. The red diamonds represent the difference between the line profiles of the non-magnetic run and the 500 G-run without Zeeman effect (see text); the blue squares represent the difference between the $500 \mathrm{G}$-run profiles with and without Zeeman effect; solid lines connect the symbols representing profiles with $v_{\text {rot }} \sin i=7.5 \mathrm{~km} \mathrm{~s}^{-1}\left(\alpha=0.2, i=60^{\circ}\right)$, dotted lines connect symbols representing profiles without rotation. All values were normalised by the integrated difference between the non-magnetic and the $500 \mathrm{G}$ run profile (with Zeeman effect).

lines.

Instead of using the integrated difference between the profiles, one can also calculate the impact on parameters describing the line profile. Figure 6.25 shows the differences of the four line profile shape parameters equivalent width $(\mathrm{EW})$, full width at half maximum (FWHM), Doppler shift at of the line core, and of the line wings (cf. Sects. 6.2.2 and 6.3.2) between differently obtained profiles of the Fe I line at $617.3 \mathrm{~nm}$ for all spectral types considered. The colour code is analogous to Fig. 6.24: Figures B.27 and B.28 in Appendix B show the corresponding plots for the other two spectral lines considered. For the EW and the FWHM the effect of the thermodynamic modifications is usually negative (shallower and narrower lines), while the impact of the Zeeman effect is positive (broader lines). The effect on the effective Doppler shifts is more complicated and highly dependent on rotation velocity. Again, the effect of the modified thermodynamical structure is negligible only in the M-star simulations, while it is dominant in F- and G-type stars.

\subsection{Test case: two-component measurement of the mag- netic field}

In Sect. 6.3, the effects of the magnetic field on the profiles of spectral lines were investigated. In this section, it will be shown that these effects can impair the measurement of the magnetic field. A simple two-component model is applied in order to fit the two magneti- 

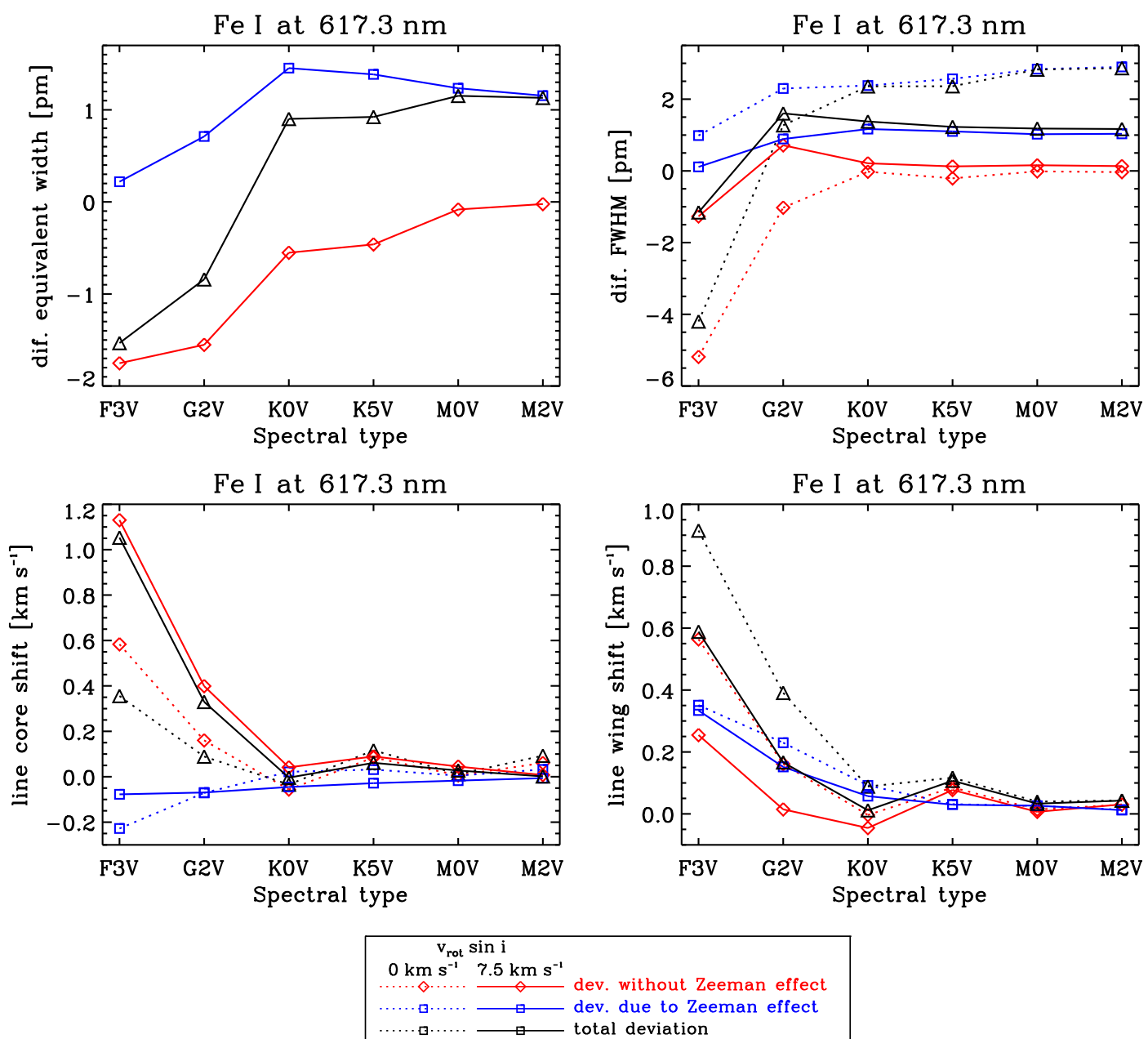

Figure 6.25: Differences between parameters of differently obtained line profiles. The black triangles represent the differences between the profiles of the $500 \mathrm{G}$ runs with Zeeman effect and the profiles of the non-magnetic runs; the red diamonds represent the difference between the line profiles of the 500 G-run without Zeeman effect (see text) and of the non-magnetic run ; the blue squares represent the difference between the $500 \mathrm{G}$ run profiles with and without Zeeman effect; solid lines connect the symbols representing profiles with $v_{\mathrm{rot}} \sin i=7.5 \mathrm{~km} \mathrm{~s}^{-1}\left(\alpha=0.2, i=60^{\circ}\right)$, dotted lines connect symbols representing profiles without rotation.

cally most sensitive of the lines considered (i. e. the Ti I line at $2223.3 \mathrm{~nm}$ and the Fe I line at $617.3 \mathrm{~nm}$ ) in the $500 \mathrm{G}$ run of the K0V star simulation. This model assumes that the magnetic structures on the stellar surface have a magnetic field strength, $B$, and a filling factor, $f$. The remaining surface fraction, $1-f$, is assumed to be field-free. Consequently, the spectral line can be fitted by a superposition of the spectral line originating from the magnetic component, weighted with $f$, and from a non-magnetic component, weighted with $1-f$. Usually, the input spectral lines for this method originate from 1D models (e. g. Anderson et al. 2010). However, in this test case, the input spectral lines were obtained by a full $3 \mathrm{D}$ calculation including disc integration. The following two setups were used: 
- Setup 1 uses one snapshot of the non-magnetic K0V simulation as input, i.e. the influence of the magnetic field on the atmospheric structure is neglected. The line calculation with SPINOR was performed on the 3D structure $(T, p, \varrho, v)$, assuming a homogeneous vertical (height-independent) field with various strengths between $0 \mathrm{G}$ and $3 \mathrm{kG}$ to generate a "grid" of input spectra. The step in the grid is $250 \mathrm{G}$. Between these points, an interpolation was used. Tests yielded a precision of $\pm 10 \mathrm{G}$ for this interpolation.

- Setup 2 uses one snapshot of the K0V simulation with $B_{0}=500 \mathrm{G}$ as input. The area was divided into magnetic and non-magnetic parts according to a reference (vertical) optical depth, $\tau_{\mathrm{R}, \text { ref }}$, and a threshold value of the magnetic field, $B_{\text {ref }}$ : every point $(x, y)$ with $B\left(\tau_{\text {ref }}, x, y\right)>B_{\text {ref }}$ belongs to the magnetic part of the atmosphere and all other points to the non-magnetic part. However, instead of the inhomogeneous magnetic field (simulation result), a homogeneous, vertical (height-independent) magnetic field of various strengths between $0 \mathrm{G}$ and $3 \mathrm{kG}$ was assumed to calculate the line profile from the magnetic component, while the non-magnetic line component was obtained from the non-magnetic part of the box with the field set to 0. Considering the formation height of the lines (which range from about $\log \tau_{\mathrm{R}}=-0.5$ to $\log \tau_{\mathrm{R}}=-3.0$ in this star) and the bimodal distribution of the magnetic field (cf. Figs. 3.17 and 3.18 in Sect. 3.2), a reasonable choice for the two reference values is $\left(B_{\text {ref }}, \log \tau_{\mathrm{R}, \text { ref }}\right)=(500 G,-1.5)$, which will be applied in the following.

For simplicity, the inclination, $i$, the differential rotation parameter, $\alpha$, and the rotational velocity $v_{\text {rot }}$ were assumed to be known $\left(i=60^{\circ}, \alpha=0.2, v_{\text {rot }}=5.77 \mathrm{~km} \mathrm{~s}^{-1}\right.$, hence $v_{\text {rot }} \sin i=5 \mathrm{~km} \mathrm{~s}^{-1}$ ). For real measurements, these parameters are additional free parameters to be fitted. After the superposition of the line profiles of the two components, a wavelength shift corresponding to a Doppler shift of up to $200 \mathrm{~m} \mathrm{~s}^{-1}$ was allowed to optimise the fit.

Figure 6.26 shows the result of the two setups in the $B$ - $f$-plane along with an approximation to the simulation result (from which the spectral line originates). As the real simulation result (as well as a real star) is not fully described by a height-independent two-component model, there is no ideal solution of the fitting parameters $B$ and $f$. The black curve shown in the figure represents the mean of $B$ where $B\left(x, y, \tau_{\mathrm{R}}\right)>500 G$ and the area fraction where $B\left(x, y, \tau_{\mathrm{R}}\right)>500 G$ for $0 \geq \log \tau_{\mathrm{R}} \geq-3$. For the rest of this section, these values are just called $B$ and $f$ in analogy to the fit parameters, which try to represent them. At a first glance, Setup 1 might appear to be somewhat closer to the "real" values of $B$ and $f$. The result for the Ti I line of $(B, f)=(1.66 \mathrm{kG}, 0.28)$ is close to the $(B, f)$ of the simulation at the optical surface $(B, f)=(1.73 \mathrm{kG}, 0.32)$. The result for the iron line, $(B, f)=(1.52 \mathrm{kG}, 0.30)$ is also not too far off these values. However, one has to take into account the formation height of the spectral lines. The lines both form over a height range, but are most sensitive to the conditions in the layer around $\log \tau_{\mathrm{R}}=-1.5$. As described in Sect. 3.2, the magnetic field is strongly height-dependent. At $\log \tau_{\mathrm{R}}=-1.5$ the K0V simulation with $B_{0}=500 G$ has a lower field strength and a higher filling factor, namely $(B, f) \approx(1.19 \mathrm{kG}, 0.49)$. For the iron line, these values are matched quite nicely by Setup 2, which gives $(B, f)=(1.25 \mathrm{kG}, 0.50)$. For the Titanium line even the result of Setup 2 is quite far off: $(B, f)=(1.22 \mathrm{kG}, 0.37)$. In both cases, however, Setup 2 gives better results for $B$ and $f$ than Setup 1 . 


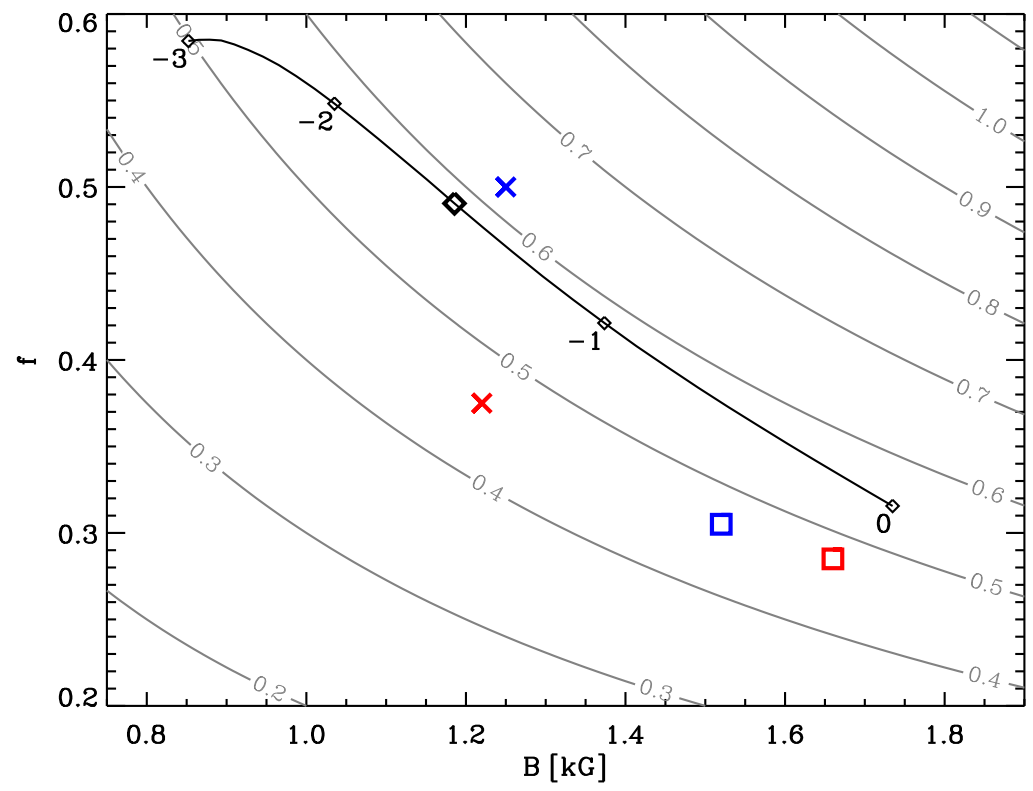

Figure 6.26: Result of two-component fits of spectral lines from the K0V simulation with $B_{0}=500 \mathrm{G}$ in the $B-f$ plane. Setup 1 is indicated by squares, setup 2 by crosses; blue symbols are the fit results for the Fe I line at $617.3 \mathrm{~nm}$, red symbols for the Ti I line at $2223.3 \mathrm{~nm}$. The black curve represents the run of $\left\langle B\left(x, y, \tau_{\mathrm{R}}\right)\right\rangle_{B\left(x, y, \tau_{\mathrm{R}}\right)>500 \mathrm{G}}$ and $f\left(B\left(x, y, \tau_{\mathrm{R}}\right)>500 \mathrm{G}\right)$ in the simulation from $\log \tau_{\mathrm{R}}=0$ to $\log \tau_{\mathrm{R}}=-3$ (some values are indicated). Grey contours indicate curves of constant $B f$. The big diamond symbol corresponds to the approximate position of the simulation in the $B-f$ plane (further explanations in the text).

Often, instead of $(B, f)$, the product $B f$ is given for measurements in real stars, as it is supposed to be more stable (cf. Reiners 2012). In Fig. 6.26 curves of constant $B f$ are indicated. The "real value" of $B f$ is between 500 and $600 \mathrm{G}$ (owing to local small-scale patches of reversed polarity it is not exactly $500 \mathrm{G}$ ) and almost independent of height. The value at $\log \tau_{\mathrm{R}}=-1.5$ is $581 \mathrm{G}$. The two-component fits generally underestimate $B f$ by $20-30 \%$. The only exception is the Fe I line in Setup $2(B f=625 \mathrm{G})$. For the measurement of $B f$, both setups have similar errors.

As discussed in Sect. 6.3.4, the effects of the modified thermodynamical structure are strongest in the F3V and G2V star. Hence, the test case presented in this section for the K0V star does not present the most extreme case of a systematic error in a magnetic field measurement introduced by line weakening, modified convective flows, and optical surface structure. But even in the moderate case of the K0V star, the field is overestimated by more than $30 \%$, and the filling factor is underestimated by roughly $40 \%$. As these effects can be explained by the unaccounted 3D effects in Setup 1 (which are partly included in Setup 2), it seems rather coincidental that the values obtained with Setup 1 are relatively close to the values of $B$ and $f$ at the optical surface (where the line shows almost no response to the magnetic field) and that the fit value of $B f$ underestimates the value most closely representing the simulation by only $20 \%$. It is beyond the scope of this thesis, to 
analyse the impact on magnetic field measurements in more detail and for other spectral types. 


\section{Conclusion}

\subsection{Brief summary of the results}

In this thesis, the near-surface convection of main-sequence stars with spectral types F3V to $\mathrm{M} 2 \mathrm{~V}$ was analysed. Special emphasis was given to the effect of a moderate magnetic field (signed average of up to a $500 \mathrm{G}$ ) on the convection. In the following, the main results are listed:

- All simulations show qualitatively similar convection patterns at and below the optical surface with hot extended upflows and a network of fast, cool downflows.

- Although the mechanisms that drive and shape the convection are similar in all simulations, the differences in stellar parameters (a factor of 5 in the gravitational acceleration and a factor of 2 in effective temperature) entail differences in granule size and shape, intensity contrast, flow velocities, superadiabaticity, etc. The highly non-linear temperature dependence of the opacity (in particular its low temperature sensitivity between 4000 and $5000 \mathrm{~K}$ ) causes some differences in the atmospheric structure (e.g. "hidden" granulation) in stars cooler than the Sun and is responsible for a strong limb darkening and bright vertical vortex structures in $\mathrm{K}$ stars.

- The magnetic field is advected into the downflow lanes where it is compressed by the flows. The magnetic flux concentrations impede horizontal inflows into the downflows and become partially evacuated; the external gas pressure is balanced by the sum of gas and magnetic pressures in the flux concentrations. This evacuation entails local depressions of the optical surface. The depth of these depressions is strongly decreasing along the model sequence from hot to cool stars. The typical field strength in the magnetic flux concentrations amounts to a few $\mathrm{kG}$ (at the optical surface) and is rather independent of the amount of flux available and of stellar parameters (within the considered parameter range).

- The magnetic flux concentrations appear as bright or dark structures depending on their size and the position on the stellar disc. On F, G, and K stars, small flux concentrations are bright because they are radiatively heated through their side walls. This mechanism is inefficient if the radius-to-depth ratio is large. Larger flux concentrations therefore appear dark on F, G, and K stars. On M stars the local depressions of the optical surface in the magnetic flux concentrations are rather shallow and most of the magnetic flux concentrations appear as dark regions. 
- For the non-magnetic simulations the limb darkening does not strongly deviate from the limb darkening of a 1D atmosphere of the same spectral type. The limb darkening strongly differs between magnetic and non-magnetic simulations: as even the larger magnetic flux concentrations become bright near the limb, the limb darkening is reduced in the magnetic simulations.

- The profiles of three spectral lines were calculated for several snapshots of each simulation. For disc-integrated spectra, a small influence of differential rotation on the shape of the line profiles was found. In the magnetic simulations, the line profiles are affected not only by the Zeeman effect, but also by the modified flows and thermodynamical structure in the magnetic flux concentrations.

- In a test case it was shown that the magnetic field is overestimated by two-component models which neglect the effects of the magnetic field on the thermodynamical structure.

\subsection{Relevance for stellar physics}

For the Sun, it has been shown that comprehensive 3D MHD simulations can help to interpret observational data and that the observations can, to some extent, provide evidence for the validity of the simulations (cf. Carlsson et al. 2004, Keller et al. 2004, Shelyag et al. 2007, Cheung et al. 2008, Bello González et al. 2009). For stars other than the Sun, where no spatially resolved observations are available, such a comparison between observations and simulations is much more difficult and consequently the way in which one can learn from it is less direct. Nonetheless, there are various implications of the results presented in this thesis for stellar astrophysics.

The simulation results presented here were calculated with a unipolar and initially vertical magnetic field. For solar simulations this is the typical numerical setup to model mature plage regions or weakly magnetised "quiet sun" (network and intranetwork) without strong emergence of magnetic flux (cf. Vögler et al. 2005). Although large parts of the solar photosphere can be described by this setup, the emergence of new flux is essential in the formation of active regions with bipolar structure and of sunspots (cf. Cheung et al. 2010, Stein and Nordlund 2012). It is very likely that flux emergence takes place on other stars as well, and many observations indicate the existence of temperature inhomogeneities interpreted as starspots on stars of different types (cf. Strassmeier 2009, Reinhold et al. 2013). More simulations of various setups are thus needed to study these different aspects of stellar magnetism (cf. Sect. 7.3).

Consequently, the results presented in this thesis are just a first step towards understanding stellar surface magnetoconvection. The integrated light from the Sun is, for instance, more strongly influenced by small-scale magnetic features like magnetic bright points or faculae than by the relatively rare sunspots (cf. Ball et al. 2012). For rather inactive stars, the magnetoconvection of plage and "quiet-sun" regions is hence probably a very important aspect of the magnetic structuring of the photosphere.

Although the simulations presented here only show parts of the magnetic phenomena which are structuring the atmospheres of stars, they provide insights in the relevant physical processes which influence the observable signals and help to estimate the magnitude 
of this influence. In the following, I outline a few examples which show the importance of the results presented, which also motivates the outlook presented in Sect. 7.3:

- Magnetic field measurements using Stokes I. Recently, many measurements of the magnetic field in various types of stars have been reported (see Reiners 2012). Most of these measurements neglect a possible correlation between magnetic field strength and atmospheric structure and just assume a 1D atmosphere with a heightindependent magnetic field, which only acts on the spectral lines by virtue of the Zeeman effect. Although this method can reproduce some observed spectra very well (cf. Reiners and Basri 2007), the shortcomings of its assumptions are obvious: all local modifications in the atmospheric structure caused by the magnetic field are disregarded. It has been shown in this thesis that, depending on the large-scale distribution of the magnetic field and especially on the spectral type and the spectral lines used, these modifications can become crucial. But there are also cases (e.g. the M stars at moderate field strength), where the impact is comparatively small. The results presented in this thesis and the future work based on this project can thus help to distinguish between more and less reliable magnetic field measurements on physical grounds and help to improve these measurements by the possible inclusion of some relevant indirect effects of the field on the spectral lines.

- Spectropolarimetry and Zeeman Doppler imaging (ZDI). Similarly to the StokesI measurement of the magnetic field, also ZDI generally assumes the same atmospheric structure for all values of the magnetic field strength. Rosén and Kochukhov (2012) showed that the reconstruction of magnetic fields in cool spots with ZDI fails if the temperature is not simultaneously reconstructed. However, the results presented in Sect. 3.3 show that even a magnetic field distribution which is homogeneous on scales resolved by ZDI can result in correlations between $T$ and $B$ (on scales much below the resolution of a spatial temperature reconstruction). Moreover, the magnetic field is strongly correlated with the velocity field, which means that the spatial resolution of ZDI (which relies on the Doppler effect), is reduced. With the disc-integration method described in Sect. 6.1 and a set of simulations with different magnetic field strength and direction, it is now possible to provide artificial time-dependent spectropolarimetric data with which ZDI reconstruction can be tested and possibly improved.

- Calibration of stellar parameters and abundances. The difference in limb darkening or spectral line profile shape between magnetic and non-magnetic simulation runs of the same spectral type is often comparable to the difference between different spectral types (without magnetic field). Even if the magnetic field is not detectable, it might strongly influence spectral classification and abundance analysis. Asplund et al. (2009) showed that taking into account the effects of three-dimensional hydrodynamical simulations (rather than a one-dimensional atmosphere) already has a strong impact (about 0.2 dex in heavy elements) on the deduced elemental abundances of the Sun. Including a magnetic field might have an additional effect of similar magnitude on stars. The results presented in this thesis with the comparatively coarse treatment of radiative transfer might not be ideal for detailed abundance analysis, the simulation results obtained can perhaps still 
improve the spectral classification and determination of parameters of active stars as they indicate, which changes in the observable signals are to be expected if the star harbours a substantial magnetic field.

- Exoplanet detection and characterisation. Active stars are much more spectroscopically and photometrically variable than inactive stars. The so-called stellar jitter caused by magnetic fields can impair the detection and characterisation of extrasolar planets (cf. Cegla et al. 2013). Sect. 6.3.3 illustrates that the modifications of the convective flows can induce radial velocity signals in the disc-integrated spectra, which probably vary with rotation phase if the magnetic field distribution in stellar longitude is inhomogeneous. These signals have to be understood and disentangled from signals that might be caused by the orbital motion of a planetary companion. Moreover, the magnetic field has a strong impact on the limb darkening (see Sect. 5.2), which is important for the characterisation of transiting extrasolar planets. In return, the multitude of planet transits of which high signal-to-noiseratio lightcurves are available (e.g. from the Kepler and CoRoT satelites), might help to measure the limb darkening of stars, which could provide insight in their magnetically induced surface properties (faculae, micropores, but also spots).

\subsection{Outlook}

The results presented are an important step towards a more detailed understanding of surface magnetism in stars. As already pointed out in Sect. 7.2, the possible applications of the results are varied and consequently there are many different directions for the future work based on this project.

One possible and very crucial direction is to extend the simulations for the stellar types already available and use different setups. This can include the modelling of starspots, flux emergence, but also the small-scale dynamo. In short, all the different setups which have been used for solar MHD in recent years can also be used on stellar MHD to complete the picture of comprehensively simulated magnetic surface phenomena.

As soon as different magnetic configurations including spots have been simulated, a detailed comparison to observational data is a relevant next step. The direct fitting of observational spectra with synthetic spectra generated from three-dimensional model atmospheres including a non-trivial global configuration of the magnetic field is a highly degenerate problem and rather beyond the scope of applications for such simulations. However, a statistical analysis of differently active stars might help to identify more and less likely magnetic field configurations. The simulations could, for instance, answer the question whether a large spot, covering a substantial fraction of the surface of a star (see Strassmeier 2009, for some examples) is more likely composed of many small units (smaller spots, pores, and/or micropores) or rather of a single large starspot. Moreover, these simulations provide a basis to test, validate, and eventually improve inversion methods used by observers (such as ZDI, cf. Sect. 7.2).

Another important step for the next few years will be to improve the code and the tables (especially for the opacities) in order to be more suitable for the conditions on stars considerably cooler and denser (or hotter and less dense) than the Sun. Cheung and Cameron (2012) extended the MURaM code to include Hall currents and ambipolar 
diffusion, the two most important effects in a weakly ionised plasma that are not covered by the MHD formalism given in Sect. 2.1.1. Other steps that might be important would be to include rotation (at least for large computational boxes), radiative heat flux through the bottom boundary (for the hot end of our model sequence), and eventually a realistic transition from a plasma to a neutral gas and possibly the formation of condensates (dust, clouds, etc.). The latter two effects will become important if one aims at modelling even cooler stars and finally brown dwarfs.

This motivates another possibility to extend the study presented here: the extension of the model sequence in the stellar parameter space. Hydrodynamical simulations (without magnetic fields) have been done for a much wider range of stellar parameters than presented in this thesis, including red giants and white dwarfs as well as very metal-poor stars (e. g. Ludwig et al. 1994, Collet et al. 2007, Trampedach et al. 2013, Magic et al. 2013). In all these stars, magnetic fields are expected to play an important role and eventually it will be necessary to run analogous simulations including magnetic fields.

There are many problems in solar and stellar physics which still are not fully solved. The list includes dynamo action for different Rossby numbers, the heating of the solar corona, the predictability of the solar cycle, magnetic star-planet interactions, the impact of stellar activity on habitability of planets, etc. In order to eventually solve at least some of these issues, it is very important to combine the results and methods from different disciplines. Comprehensive three-dimensional simulations are an ideal bridge between the idealised theory on the one hand, and the noise-afflicted, often ambiguous observationally obtained results, on the other. 



\section{Bibliography}

Allende Prieto, C., Koesterke, L., Ludwig, H.-G., Freytag, B., and Caffau, E.: 2013, Astronomy $\mathcal{F}$ Astrophysics 550, A103

Ammler-von Eiff, M. and Reiners, A.: 2012, Astronomy E Astrophysics 542, A116

Anders, E. and Grevesse, N.: 1989, Geochimica et Cosmochimica Acta 53, 197

Anderson, R. I., Reiners, A., and Solanki, S. K.: 2010, Astronomy E Astrophysics 522, A81

Asplund, M., Grevesse, N., Sauval, A. J., and Scott, P.: 2009, Annual Review of Astronomy $\mathcal{G}$ Astrophysics 47, 481

Asplund, M., Nordlund, A., Trampedach, R., Allende Prieto, C., and Stein, R. F.: 2000, Astronomy E Astrophysics 359, 729

Bachmann, K. T., Maymani, H., Nautiyal, K., and te Velde, V.: 2004, Advances in Space Research 34, 274

Ball, W. T., Unruh, Y. C., Krivova, N. A., Solanki, S., Wenzler, T., Mortlock, D. J., and Jaffe, A. H.: 2012, Astronomy E Astrophysics 541, A27

Beeck, B., Cameron, R. H., Reiners, A., and Schüssler, M.: 2013a, Astronomy E Astrophysics 558, A48

Beeck, B., Cameron, R. H., Reiners, A., and Schüssler, M.: 2013b, Astronomy E Astrophysics 558, A49

Beeck, B., Collet, R., Steffen, M., Asplund, M., Cameron, R. H., Freytag, B., Hayek, W., Ludwig, H.-G., and Schüssler, M.: 2012, Astronomy E Astrophysics 539, A121

Beeck, B., Schüssler, M., and Reiners, A.: 2011, in C. Johns-Krull, M. K. Browning, and A. A. West (eds.), 16th Cambridge Workshop on Cool Stars, Stellar Systems, and the Sun, Vol. 448 of ASPC, p. 1071

Bello González, N., Yelles Chaouche, L., Okunev, O., and Kneer, F.: 2009, Astronomy E Astrophysics 494, 1091

Bessell, M. S.: 1990, Astronomical Society of the Pacific 102, 1181

Bharti, L., Beeck, B., and Schüssler, M.: 2010, Astronomy $\mathcal{E}$ Astrophysics 510, A12 
Biskamp, D.: 1997, Nonlinear Magnetohydrodynamics

Bonet, J. A., Márquez, I., Sánchez Almeida, J., Cabello, I., and Domingo, V.: 2008, The Astrophysical Journal 687, L131

Bonet, J. A., Márquez, I., Sánchez Almeida, J., Palacios, J., Martínez Pillet, V., Solanki, S. K., del Toro Iniesta, J. C., Domingo, V., Berkefeld, T., Schmidt, W., Gandorfer, A., Barthol, P., and Knölker, M.: 2010, The Astrophysical Journal 723, L139

Bovelet, B. and Wiehr, E.: 2001, Solar Physics 201, 13

Bray, R. J. and Loughhead, R. E.: 1964, Sunspots

Bressan, A., Marigo, P., Girardi, L., Salasnich, B., Dal Cero, C., Rubele, S., and Nanni, A.: 2012, Monthly Notices of the Royal Astronomical Society 427, 127

Cameron, R., Schüssler, M., Vögler, A., and Zakharov, V.: 2007, Astronomy $\mathcal{F}$ Astrophysics 474, 261

Carlson, B. G.: 1963, in B. Alder and S. Fernbach (eds.), Methods in Computational Physics, Vol. 1 of Methods in Computational Physics, pp 1-42

Carlsson, M., Stein, R. F., Nordlund, Å., and Scharmer, G. B.: 2004, The Astrophysical Journal 610, L137

Cegla, H. M., Shelyag, S., Watson, C. A., and Mathioudakis, M.: 2013, The Astrophysical Journal 763, 95

Chamel, N., Fantina, A. F., and Davis, P. J.: 2013, Physical Review D 88, 081301

Chen, P. F.: 2011, Living Reviews in Solar Physics 8, 1

Cheung, M. C. M. and Cameron, R. H.: 2012, The Astrophysical Journal 750, 6

Cheung, M. C. M., Rempel, M., Title, A. M., and Schüssler, M.: 2010, The Astrophysical Journal 720, 233

Cheung, M. C. M., Schüssler, M., and Moreno-Insertis, F.: 2007, Astronomy $\mathcal{E}$ Astrophysics 461, 1163

Cheung, M. C. M., Schüssler, M., Tarbell, T. D., and Title, A. M.: 2008, The Astrophysical Journal 687, 1373

Cho, K.-S., Bong, S.-C., Chae, J., Kim, Y.-H., and Park, Y.-D.: 2010, The Astrophysical Journal 723, 440

Choudhuri, A. R.: 1998, The physics of fluids and plasmas : an introduction for astrophysicists /

Claret, A.: 2000, Astronomy E Astrophysics 363, 1081

Collet, R., Asplund, M., and Trampedach, R.: 2007, Astronomy E Astrophysics 469, 687 
Collet, R., Hayek, W., Asplund, M., Nordlund, Å., Trampedach, R., and Gudiksen, B.: 2011, Astronomy $\mathcal{E}$ Astrophysics 528, A32

Das, U. and Mukhopadhyay, B.: 2012, Physical Review D 86, 042001

Del Moro, D.: 2004, Astronomy E Astrophysics 428, 1007

Donati, J.-F., Collier Cameron, A., Semel, M., Hussain, G. A. J., Petit, P., Carter, B. D., Marsden, S. C., Mengel, M., López Ariste, A., Jeffers, S. V., and Rees, D. E.: 2003, Monthly Notices of the Royal Astronomical Society 345, 1145

Dravins, D.: 1982, Annual Review of Astronomy \& Astrophysics 20, 61

Fan, Y.: 2009, Living Reviews in Solar Physics 6, 4

Frutiger, C.: 2000, Diss. ETH 13896

Frutiger, C., Solanki, S. K., Fligge, M., and Bruls, J. H. M. J.: 2000, Astronomy $\mathcal{E}$ Astrophysics 358, 1109

Gray, D. F.: 2005, The Observation and Analysis of Stellar Photospheres

Güdel, M.: 2007, Living Reviews in Solar Physics 4, 3

Hathaway, D. H.: 2010, Living Reviews in Solar Physics 7, 1

Hennebelle, P. and Teyssier, R.: 2008, Astronomy E Astrophysics 477, 25

Hirzberger, J., Bonet, J. A., Vázquez, M., and Hanslmeier, A.: 1999, The Astrophysical Journal 515, 441

Hirzberger, J., Vazquez, M., Bonet, J. A., Hanslmeier, A., and Sobotka, M.: 1997, The Astrophysical Journal 480, 406

Howard, R. and Harvey, J.: 1970, Solar Physics 12, 23

Jafarzadeh, S., Solanki, S. K., Feller, A., Lagg, A., Pietarila, A., Danilovic, S., Riethmüller, T. L., and Martínez Pillet, V.: 2013, Astronomy \& Astrophysics 549, A116

Jeffers, S. V., Barnes, J. R., Jones, H., and Pinfield, D.: 2013, in European Physical Journal Web of Conferences, Vol. 47 of European Physical Journal Web of Conferences, p. 9002

Johansen, A.: 2009, in K. G. Strassmeier, A. G. Kosovichev, and J. E. Beckman (eds.), IAU Symposium, Vol. 259 of IAU Symposium, pp 249-258

Johns-Krull, C. M., Valenti, J. A., and Saar, S. H.: 2004, The Astrophysical Journal 617, 1204

Johnson, H. L. and Morgan, W. W.: 1951, The Astrophysical Journal 114, 522

Keller, C. U.: 1992, Nature 359, 307 
Keller, C. U., Schüssler, M., Vögler, A., and Zakharov, V.: 2004, The Astrophysical Journal 607, L59

Kuelebi, B., Jordan, S., Euchner, F., Gaensicke, B. T., and Hirsch, H.: 2010, VizieR Online Data Catalog 350, 61341

Kunasz, P. and Auer, L. H.: 1988, Journal of Quantitative Spectroscopy and Radiative Transfer 39, 67

Kupka, F., Piskunov, N., Ryabchikova, T. A., Stempels, H. C., and Weiss, W. W.: 1999, Astronomy E Astrophysics Supplement 138, 119

Kurucz, R.: 1993, ATLAS9 Stellar Atmosphere Programs and 2 km/s grid. Kurucz CDROM No. 13. Cambridge, Mass.: Smithsonian Astrophysical Observatory, 1993. 13

Kurucz, R. L.: 1979, Astrophysical Journal Supplement Series 40, 1

Ludwig, H.-G.: 1992, Ph.D. thesis, Christian-Albrechts-Universität zu Kiel

Ludwig, H.-G.: 2007, Astronomy $\mathcal{E}$ Astrophysics 471, 925

Ludwig, H.-G., Allard, F., and Hauschildt, P. H.: 2002, Astronomy E Astrophysics 395, 99

Ludwig, H.-G., Allard, F., and Hauschildt, P. H.: 2006, Astronomy E Astrophysics 459, 599

Ludwig, H.-G., Jordan, S., and Steffen, M.: 1994, Astronomy E Astrophysics 284, 105

Magic, Z., Collet, R., Asplund, M., Trampedach, R., Hayek, W., Chiavassa, A., Stein, R. F., and Nordlund, Å.: 2013, Astronomy E Astrophysics 557, A26

McLaughlin, D. B.: 1924, The Astrophysical Journal 60, 22

Moll, R., Cameron, R. H., and Schüssler, M.: 2011, Astronomy E Astrophysics 533, A126

Moll, R., Cameron, R. H., and Schüssler, M.: 2012, Astronomy $\mathcal{E}$ Astrophysics 541, A68

Neckel, H. and Labs, D.: 1994, Solar Physics 153, 91

Nordlund, A.: 1982, AEFA 107, 1

Nordlund, Å. and Dravins, D.: 1990a, Astronomy $\mathcal{E}$ Astrophysics 228, 155

Nordlund, A. and Dravins, D.: 1990b, Astronomy $\mathcal{E}$ Astrophysics 228, 203

Nordlund, Å. and Stein, R. F.: 1990, in J. O. Stenflo (ed.), Solar Photosphere: Structure, Convection, and Magnetic Fields, Vol. 138 of IAU Symposium, p. 191

Nordlund, Å., Stein, R. F., and Asplund, M.: 2009, Living Reviews in Solar Physics 6, 2

Orlando, S., Peres, G., and Reale, F.: 2004, Astronomy E Astrophysics 424, 677 
Petit, P., Donati, J.-F., Oliveira, J. M., Aurière, M., Bagnulo, S., Landstreet, J. D., Lignières, F., Lüftinger, T., Marsden, S., Mouillet, D., Paletou, F., Strasser, S., Toqué, N., and Wade, G. A.: 2004, Monthly Notices of the Royal Astronomical Society 351, 826

Pietarila Graham, J., Cameron, R., and Schüssler, M.: 2010, The Astrophysical Journal 714, 1606

Piskunov, N. E., Kupka, F., Ryabchikova, T. A., Weiss, W. W., and Jeffery, C. S.: 1995, Astronomy $\mathcal{E}$ Astrophysics Supplement 112, 525

Pulkkinen, T.: 2007, Living Reviews in Solar Physics 4, 1

Ramírez, I., Allende Prieto, C., Koesterke, L., Lambert, D. L., and Asplund, M.: 2009, Astronomy \& Astrophysics 501, 1087

Rees, D. E., Durrant, C. J., and Murphy, G. A.: 1989, The Astrophysical Journal 339, 1093

Reiners, A.: 2006, Astronomy E Astrophysics 446, 267

Reiners, A.: 2012, Living Reviews in Solar Physics 9, 1

Reiners, A. and Basri, G.: 2007, The Astrophysical Journal 656, 1121

Reiners, A. and Mohanty, S.: 2012, The Astrophysical Journal 746, 43

Reiners, A., Shulyak, D., Anglada-Escudé, G., Jeffers, S. V., Morin, J., Zechmeister, M., Kochukhov, O., and Piskunov, N.: 2013, Astronomy $\mathcal{G}$ Astrophysics 552, A103

Reinhold, T., Reiners, A., and Basri, G.: 2013, Astronomy $\mathcal{G}$ Astrophysics 560, A4

Rempel, M.: 2006, The Astrophysical Journal 647, 662

Rempel, M., Schüssler, M., and Knölker, M.: 2009, The Astrophysical Journal 691, 640

Rogers, F. J.: 1994, in G. Chabrier and E. Schatzman (eds.), IAU Colloq. 147: The Equation of State in Astrophysics, p. 16

Rogers, F. J., Swenson, F. J., and Iglesias, C. A.: 1996, The Astrophysical Journal 456, 902

Rosén, L. and Kochukhov, O.: 2012, Astronomy E Astrophysics 548, A8

Rossiter, R. A.: 1924, The Astrophysical Journal 60, 15

Schrijver, C. J. and Zwaan, C.: 2000, Solar and Stellar Magnetic Activity

Schüssler, M.: 2013, in A. G. Kosovichev, E. de Gouveia Dal Pino, and Y. Yan (eds.), IAU Symposium, Vol. 294 of IAU Symposium, pp 95-106

Schüssler, M. and Vögler, A.: 2006, The Astrophysical Journal 641, L73

Schwenn, R.: 2006, Living Reviews in Solar Physics 3, 2 
Semel, M.: 1989, Astronomy E Astrophysics 225, 456

Shelyag, S., Cally, P. S., Reid, A., and Mathioudakis, M.: 2013, The Astrophysical Journal 776, L4

Shelyag, S., Schüssler, M., Solanki, S. K., and Vögler, A.: 2007, Astronomy E Astrophysics 469, 731

Solanki, S. K., Krivova, N. A., and Haigh, J. D.: 2013, Annual Review of Astronomy E Astrophysics 51, 311

Spruit, H. C.: 1976, Solar Physics 50, 269

Stein, R. F.: 2012, Living Reviews in Solar Physics 9, 4

Stein, R. F., Bercik, D., and Nordlund, A.: 2002, Nuovo Cimento C Geophysics Space Physics C 25, 513

Stein, R. F., Lagerfjärd, A., Nordlund, Å., and Georgobiani, D.: 2011, Solar Physics 268, 271

Stein, R. F. and Nordlund, A.: 1998, The Astrophysical Journal 499, 914

Stein, R. F. and Nordlund, Å.: 2000, Solar Physics 192, 91

Stein, R. F. and Nordlund, Å.: 2012, The Astrophysical Journal 753, L13

Steiner, O.: 2005, Astronomy E Astrophysics 430, 691

Steiner, O., Franz, M., Bello González, N., Nutto, C., Rezaei, R., Martínez Pillet, V., Bonet Navarro, J. A., del Toro Iniesta, J. C., Domingo, V., Solanki, S. K., Knölker, M., Schmidt, W., Barthol, P., and Gandorfer, A.: 2010, The Astrophysical Journal 723, L180

Stenflo, J. O., Gandorfer, A., Holzreuter, R., Gisler, D., Keller, C. U., and Bianda, M.: 2002, Astronomy $\mathcal{E}$ Astrophysics 389, 314

Strassmeier, K. G.: 2009, The Astronomy E Astrophysics Review 17, 251

Thuillier, G., Melo, S. M. L., Lean, J., Krivova, N. A., Bolduc, C., Fomichev, V. I., Charbonneau, P., Shapiro, A. I., Schmutz, W., and Bolsée, D.: 2013, Solar Physics

Title, A. M., Tarbell, T. D., Topka, K. P., Ferguson, S. H., Shine, R. A., and SOUP Team: 1989, The Astrophysical Journal 336, 475

Trampedach, R., Asplund, M., Collet, R., Nordlund, A., and Stein, R. F.: 2013, The Astrophysical Journal 769, 18

Trampedach, R. and Stein, R. F.: 2011, The Astrophysical Journal 731, 78

Trujillo Bueno, J., Shchukina, N., and Asensio Ramos, A.: 2004, Nature 430, 326 
Vargas Domínguez, S., Palacios, J., Balmaceda, L., Cabello, I., and Domingo, V.: 2011, Monthly Notices of the Royal Astronomical Society 416, 148

Vögler, A.: 2003, Ph.D. thesis, Gerog-August-Universität Göttingen

Vögler, A., Bruls, J. H. M. J., and Schüssler, M.: 2004, Astronomy E Astrophysics 421, 741

Vögler, A., Shelyag, S., Schüssler, M., Cattaneo, F., Emonet, T., and Linde, T.: 2005, Astronomy $\mathcal{E}$ Astrophysics 429, 335

Wedemeyer, S., Ludwig, H.-G., and Steiner, O.: 2013, Astronomische Nachrichten 334, 137

Wedemeyer-Böhm, S., Scullion, E., Steiner, O., Rouppe van der Voort, L., de La Cruz Rodriguez, J., Fedun, V., and Erdélyi, R.: 2012, Nature 486, 505 

Appendix 



\section{A Horizontal averages 1}

We consider temporally and horizontally averaged quantities to study the mean stratification of the six simulated stars and compare them to 1D models. Depending on the context, the sensible "horizontal" average is an average over surfaces of constant geometrical depth, (Rosseland) optical depth, or (gas) pressure, denoted by $\langle\cdot\rangle_{z},\langle\cdot\rangle_{\tau}$, or $\langle\cdot\rangle_{p}$, respectively. The average $\langle\cdot\rangle_{\tau}$ sensible for the layers around the optical surface and in the photosphere (especially for quantities involving the emergent intensity), while the average $\langle\cdot\rangle_{z}$ is more relevant for the convective, deeper layers, where the stratifications are nearly adiabatic and largely independent from the radiation field. However, $z$ is highly impractical as a coordinate scale for comparing simulations of stars of various spectral types, since the pressure scale height varies by more than a factor of ten between the six simulated stars presented in this paper (cf. Fig. 3.8). Therefore, we use the logarithm of the normalised pressure $\langle p\rangle_{z} / p_{0}$ as a more suitable depth coordinate (where $p_{0}=\langle p\rangle_{\tau_{\mathrm{R}}=1}$ is the average gas pressure at the optical surface) to illustrate depth dependences of quantities averaged on iso- $z$ surfaces. This should not be confused with an average on surfaces of constant pressure, $\langle\cdot\rangle_{p}$.

The averages $\langle\cdot\rangle_{\tau}$ and $\langle\cdot\rangle_{p}$ are defined as horizontal averages of a quantity on iso- $\tau$ and iso- $p$ surfaces, respectively. For the average $\langle\cdot\rangle_{p}$, which is rarely used in this article, there is the problem that sometimes iso- $p$ surfaces cannot be unambiguously defined since strong deviations from hydrostatic equilibrium in regions with Mach number of order unity can lead to a locally non-monotonic depth-dependence of the gas pressure. Our iso- $p$ surfaces are the deepest surfaces on which the pressure assumes the given value. This arbitrary choice does not significantly influence the results for the iso- $p$ means in the simulations, except for the surface layers of the F3V simulation where the flows are mostly sonic and supersonic (cf. Fig. 3.5).

Figure A.1 shows the rms fluctuations of the geometrical depth on surfaces of constant optical depth (left panel) and pressure (right panel) as a measure of the corrugation of these surfaces. Due to this corrugation of the iso- $p$ and iso- $\tau_{\mathrm{R}}$ surfaces, profiles of quantities averaged in the different ways described above are not just distorted versions of each other, but can show a significantly different depth-dependence of the same quantity. The differences between the three averaging methods are expected to be largest in the F- and G-type simulations, for which the corrugation of the iso- $p$ and iso- $\tau_{\mathrm{R}}$ surfaces is strongest.

The left panel of Figure A.2 shows a vertical cut through some of the iso- $p$ and iso- $\tau_{\mathrm{R}}$ surfaces in the G2V simulation. Note that in the optically thin upper part of the simulation domain, the iso- $p$-surfaces follow the iso- $\tau_{\mathrm{R}}$ surfaces. Although this is observed in all six

\footnotetext{
${ }^{1}$ This appendix section has been published as Appendix A in the online version of Beeck et al. (2013a)
} 

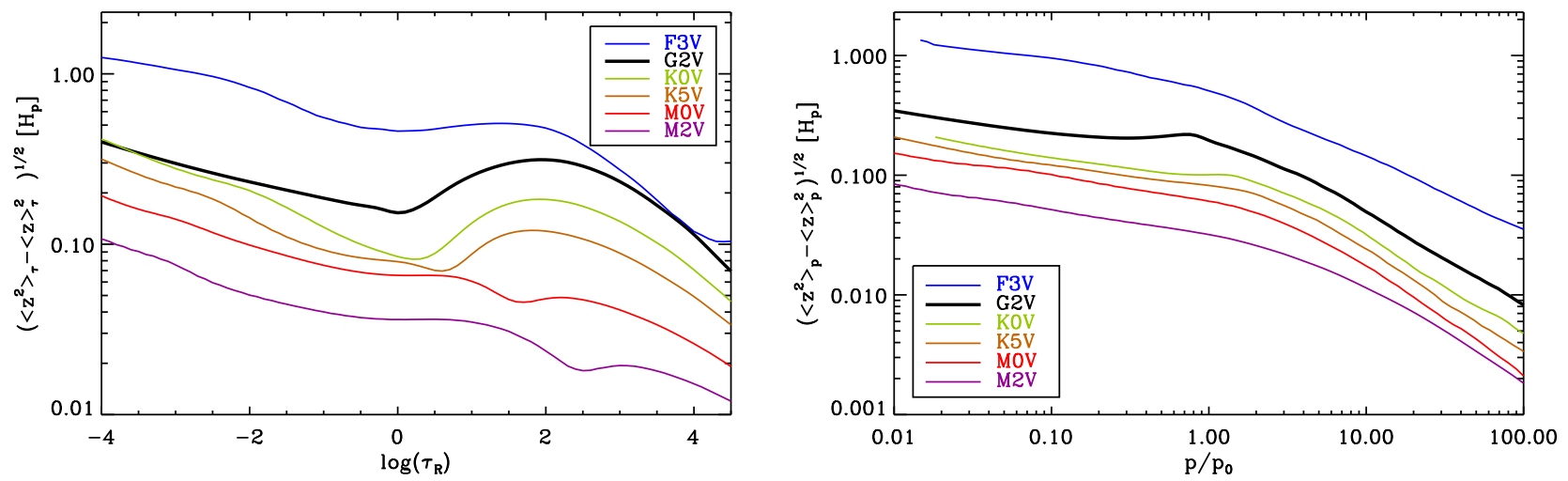

Figure A.1: Average profiles of the rms fluctuations of the geometrical height on iso- $\tau_{\mathrm{R}}$ surfaces (left) and on iso- $p$ surfaces (right) in units of the local pressure scale heights.
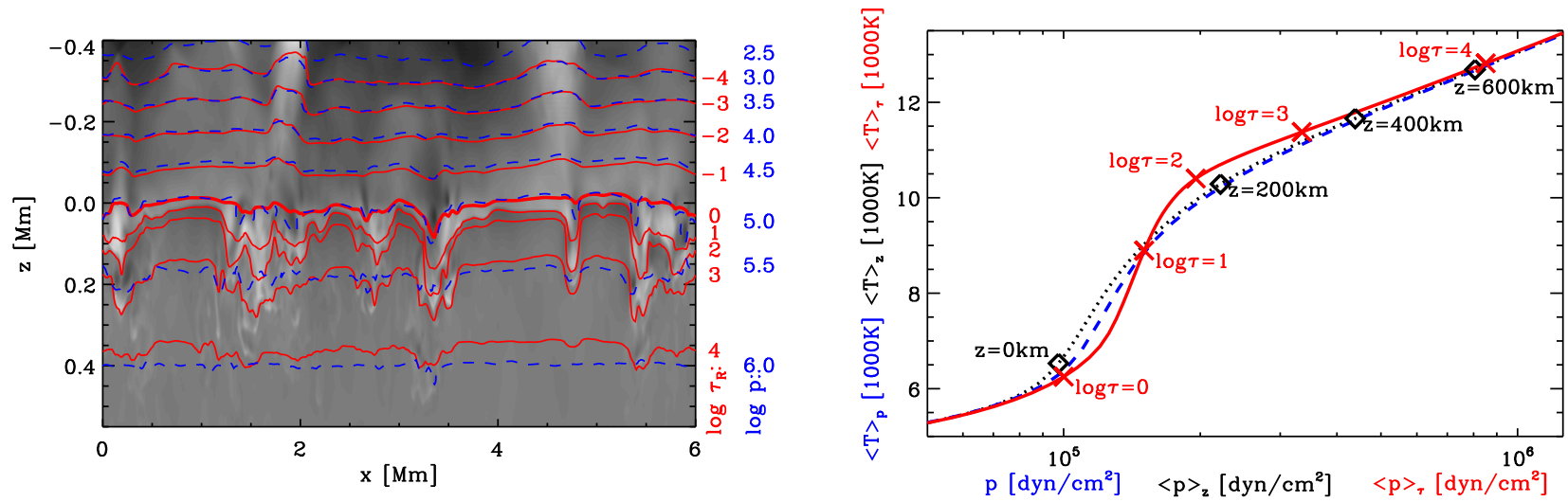

Figure A.2: Illustration of the different averages. Left panel: Vertical cut through a part of the G2V simulation domain. Surfaces of constant optical depth (solid, red curves) and constant pressure (dashed, blue curves) are indicated; the underlying grey-scale image illustrate the density (normalised by the horizontal mean $\langle\varrho\rangle_{z}$ ). Right panel: Run of the horizontally averaged temperature vs. averaged pressure in the G2V simulation for the different averages $\langle\cdot\rangle_{z}$ (dotted, black), $\langle\cdot\rangle_{\tau}$ (red,solid), and $\langle\cdot\rangle_{p}$ (blue, dashed); as a reference the position of some $z$ and $\tau$ values is marked.

simulations, this effect is most prominent in the G-type star (see discussion of Fig. 3.11 in Sect. 3.1.3). In the optically thick part, the temperature fluctuations determine the shape of the iso- $\tau_{\mathrm{R}}$ surfaces, since the opacity is highly temperature-sensitive in this regime. The iso- $p$ surfaces, however, become almost flat planes in the deeper layers since the density contrast and deviations from hydrostatic equilibrium decrease with increasing depth.

The right panel of Figure A.2 illustrates the different depth dependences of temperature, $T$, for the three different averages $\langle\cdot\rangle_{z},\langle\cdot\rangle_{\tau}$, and $\langle\cdot\rangle_{p}$. As for most of the figures in this paper, the gas pressure (here without normalisation) was used as depth coordinate. For $\langle T\rangle_{\tau}$ and $\langle T\rangle_{z}$, the pressure varies along the surfaces over which the average is per- 
formed. The depth coordinates are therefore averages themselves, namely $\langle p\rangle_{\tau}$ and $\langle p\rangle_{z}$, respectively, in these cases. As expected, the differences between the differently averaged temperature are largest at the optical surface and all three averages converge at large optical depth $\left(\log \tau_{\mathrm{R}} \gtrsim 4\right)$. As the deviations from hydrostatic equilibrium are small in the subsurface layers, $\langle\cdot\rangle_{p}$ stays close to $\langle\cdot\rangle_{z}$. The average $\langle\cdot\rangle_{\tau}$ deviates more strongly near the photospheric transition since the big temperature fluctuations govern the opacity and thus lead to strongly corrugated iso-tau surfaces. In the atmosphere, the deviations between different averages of temperature become smaller with height. Especially, $\langle T\rangle_{p} \approx\langle T\rangle_{\tau}$, in these layers as expected, because the iso- $\tau$ surfaces roughly follow the iso- $p$ surfaces.

If one aims at comparing $1 \mathrm{D}$ and averaged 3D results, one has to take into account that a 1D model does not have corrugated iso- $\tau$ surfaces. Profiles of quantities which change rapidly at the photospheric transition have a steep gradient in 1D models comparable to the local gradient in a 3D simulation. As the depth of the photospheric transition varies across the surface in a 3D simulation, these strong local gradients are smeared out and the similarity between $1 \mathrm{D}$ and averaged $3 \mathrm{D}$ results is obscured, if a plain horizontal average, $\langle\cdot\rangle_{z}$ is used. The average $\langle\cdot\rangle_{\tau}$ is more appropriate in these cases for a comparison between 1D and averaged 3D profiles. This average however has no relevance below the photospheric transition, where $\langle\cdot\rangle_{z}$ is more useful. For the stellar parameters used in our simulations, the $\langle\cdot\rangle_{p}$ average seems a good compromise between the two other averaging methods for comparison with 1D models as it is converging towards $\langle\cdot\rangle_{\tau}$ in the atmosphere and towards $\langle\cdot\rangle_{z}$ in the convection zone.

In order to obtain the temporally averaged profiles presented in this paper, first one of the horizontal averaging methods described above was applied to several snapshots with a time separation of $5-7$ minutes, depending on the star. Then, for each quantity and at each depth point, the values of the different snapshots were averaged. The timedependence of the horizontal averages of most quantities under consideration (such as $T$, $p, \varrho$, etc.) was found to be very small, so that we found a small number of snapshots to be sufficient for a sensible temporal average. The mean profiles presented in this article are averages over six snapshots each. 



\section{B Additional figures}



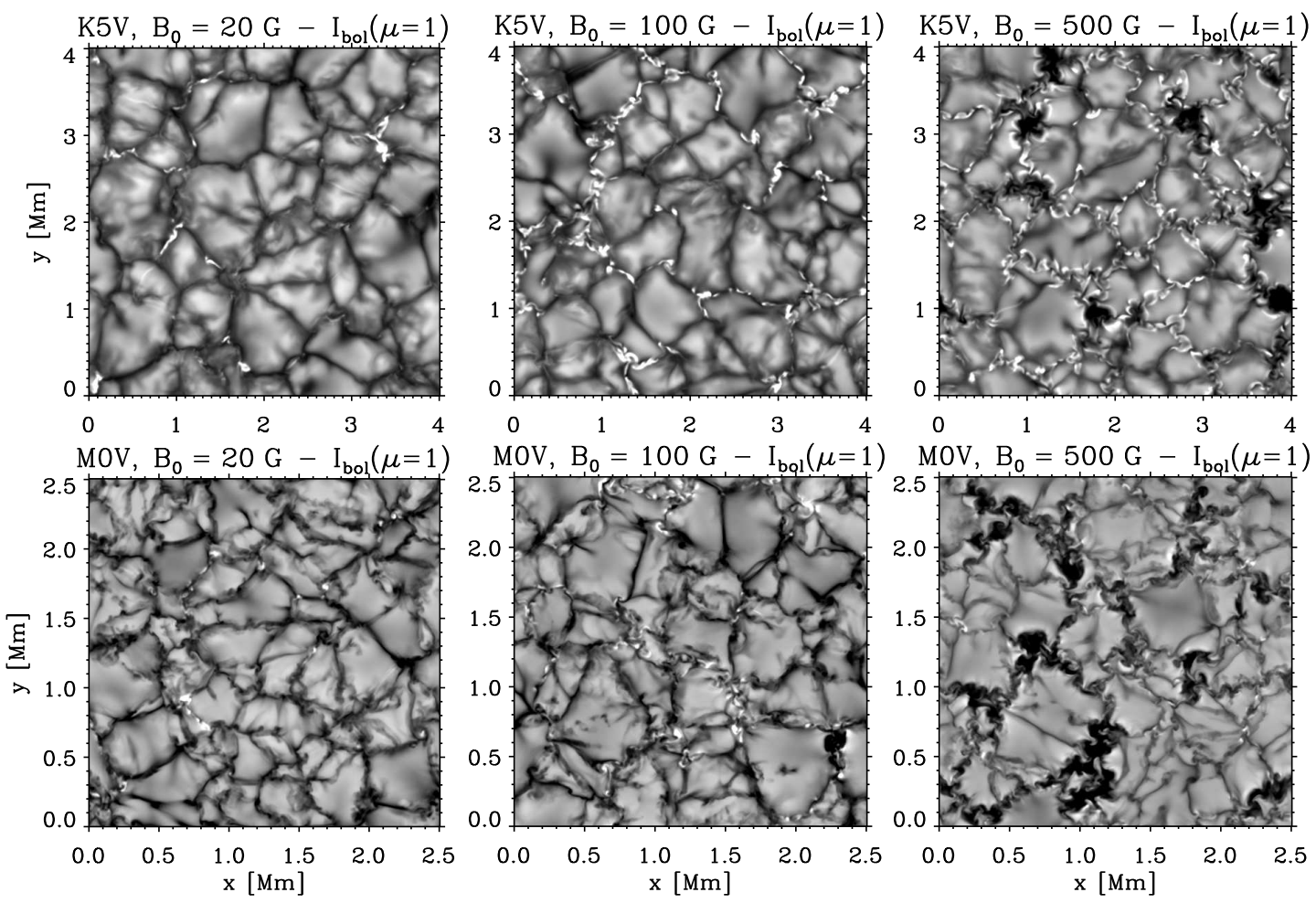

Figure B.1: Same as Fig. 3.15, but for the K5V and M0V simulations. 

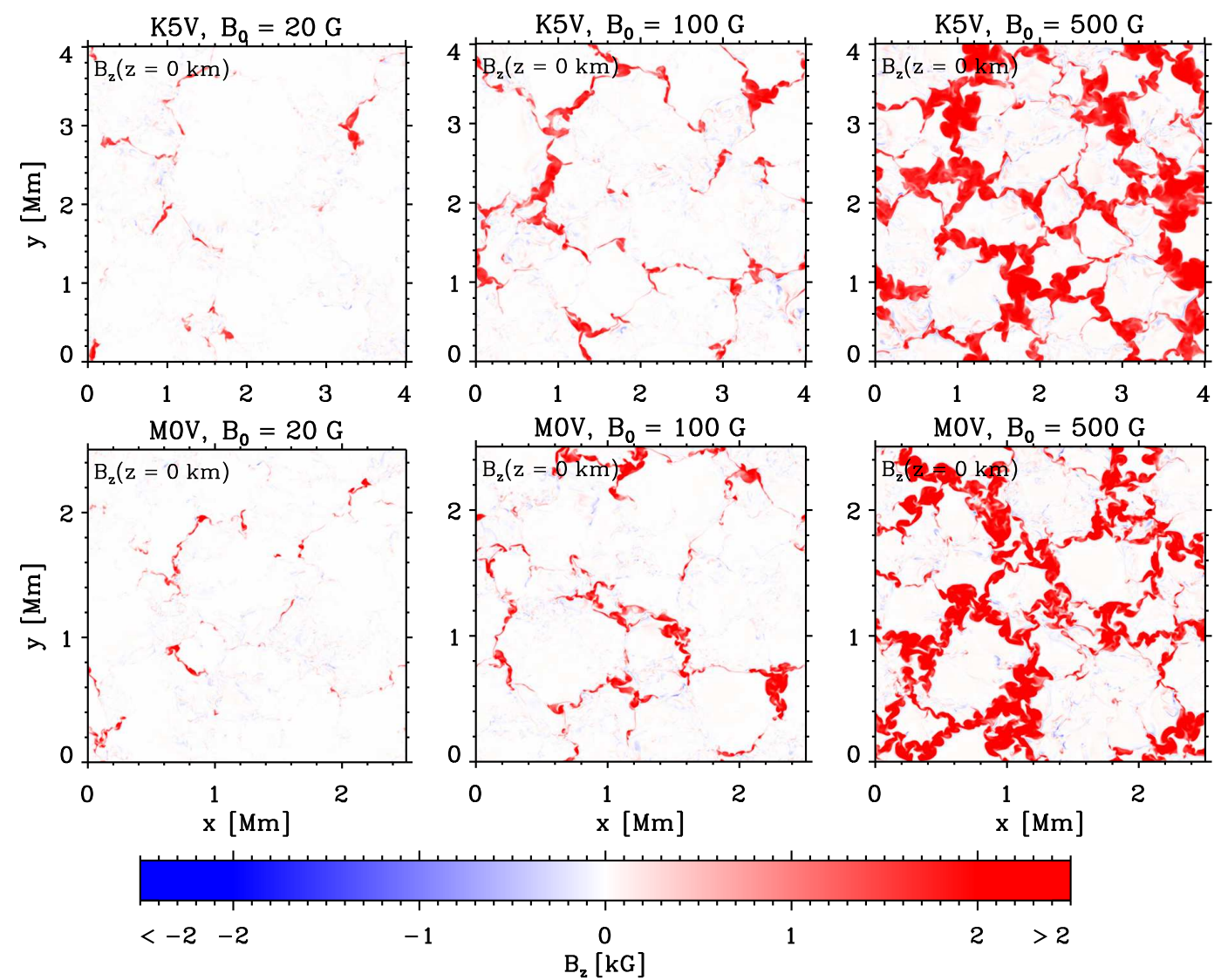

Figure B.2: Same as Fig. 3.16, but for the K5V and M0V simulations. 

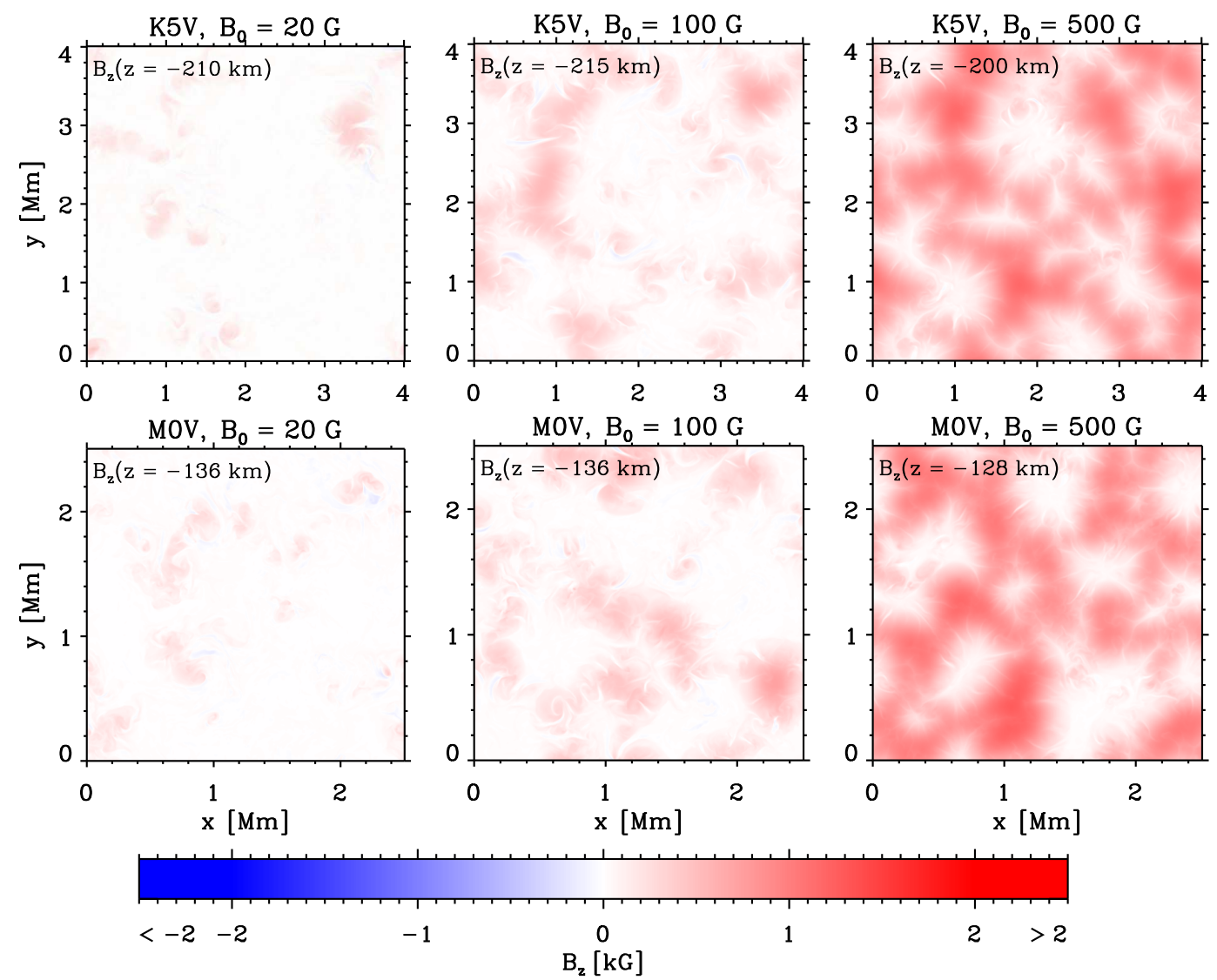

Figure B.3: Same as Fig. 3.21, but for the K5V and M0V simulations. 

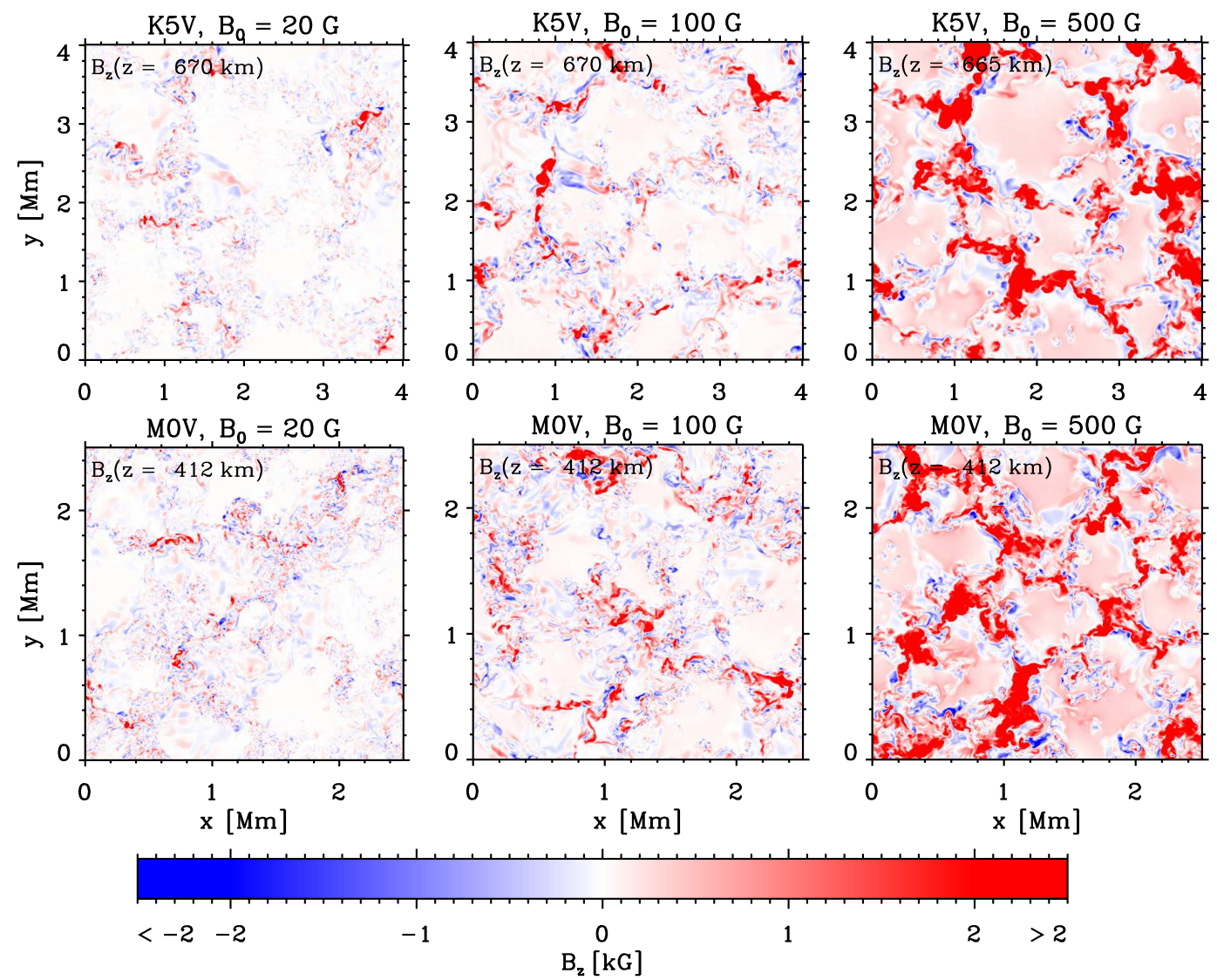

Figure B.4: Same as Fig. 3.22, but for the K5V and M0V simulations.
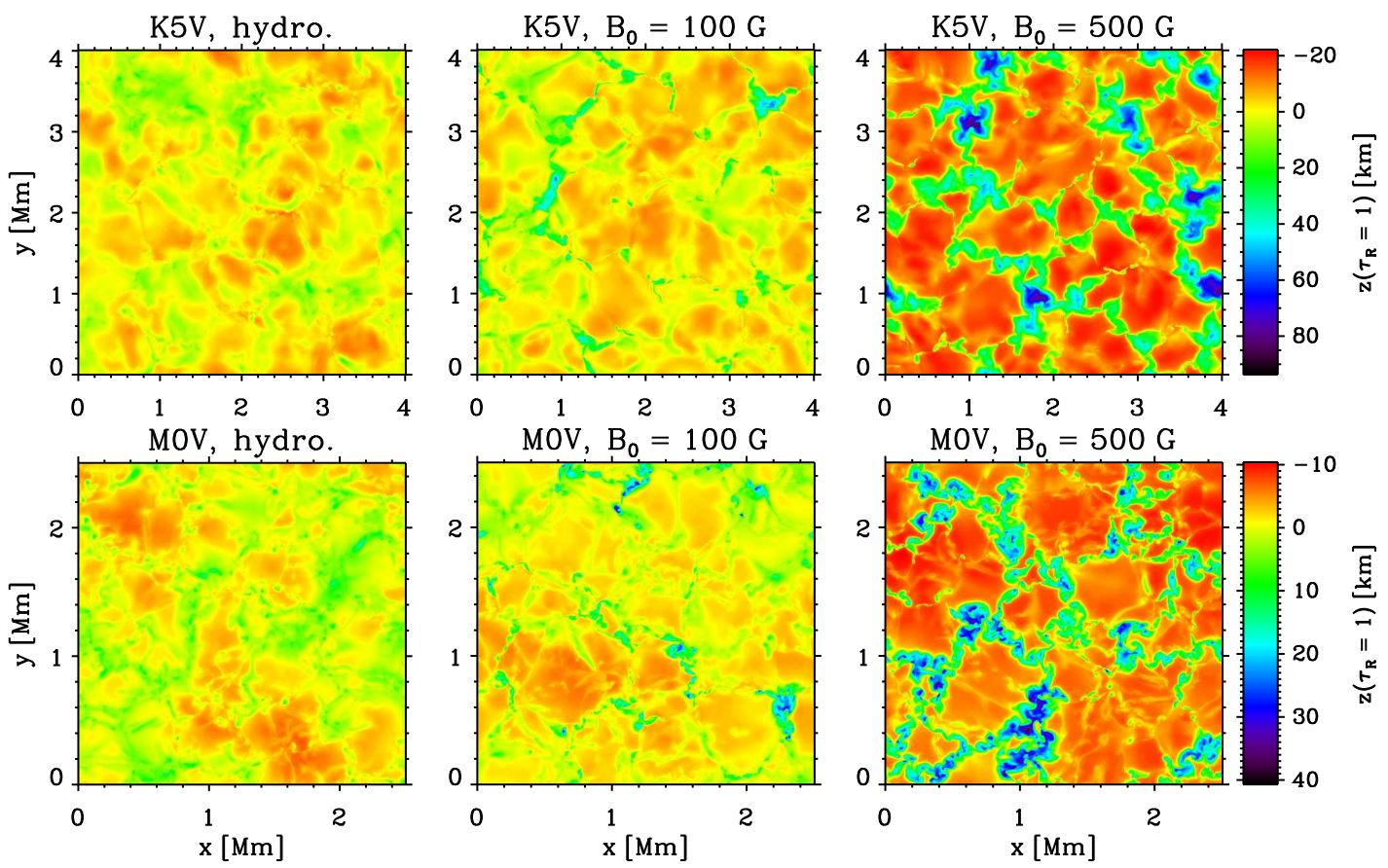

Figure B.5: Same as Fig. 3.23 but for the K5V and M2V simulations. 

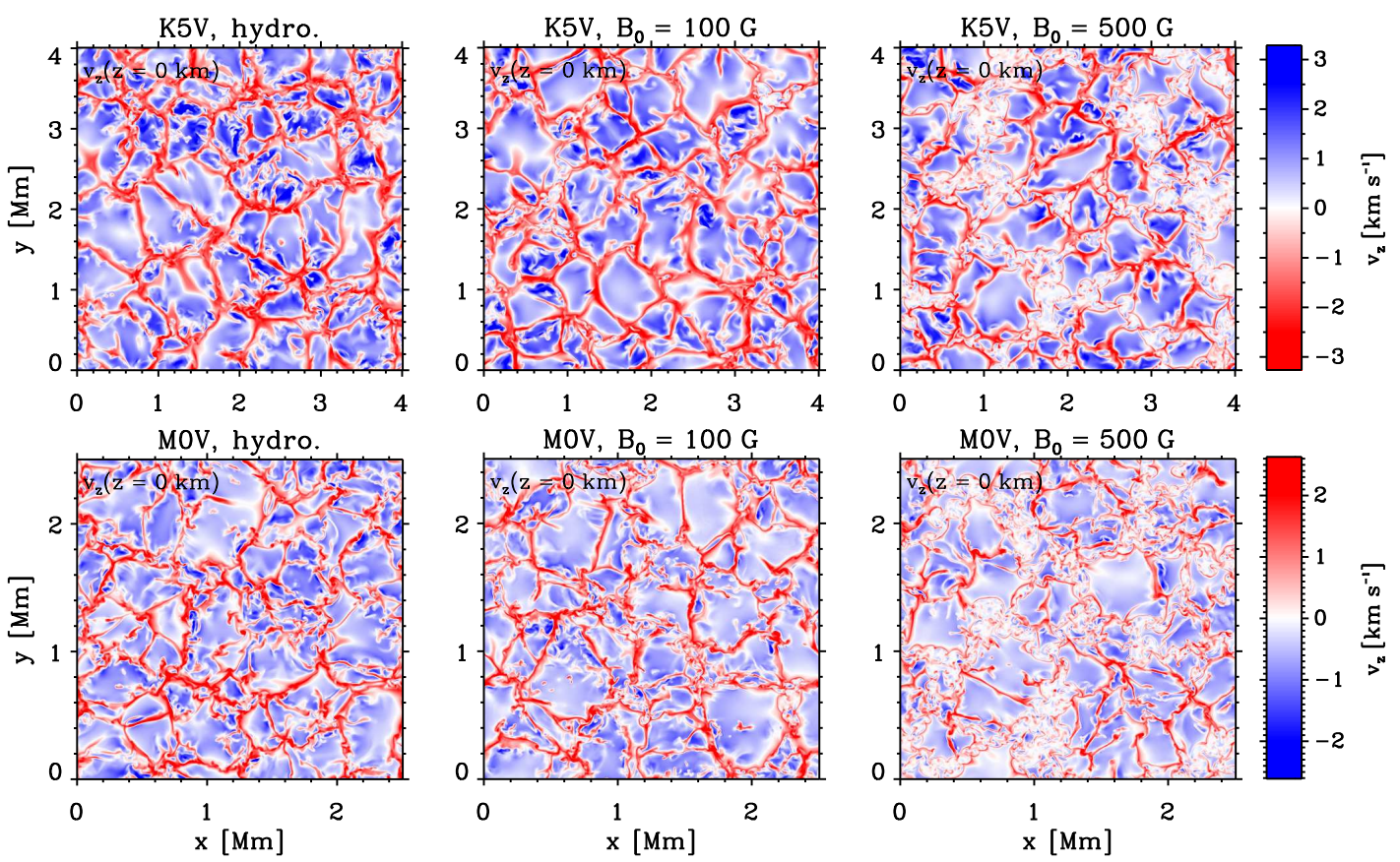

Figure B.6: Same as in Fig. 3.30, but for the K5V and M0V simulations. 

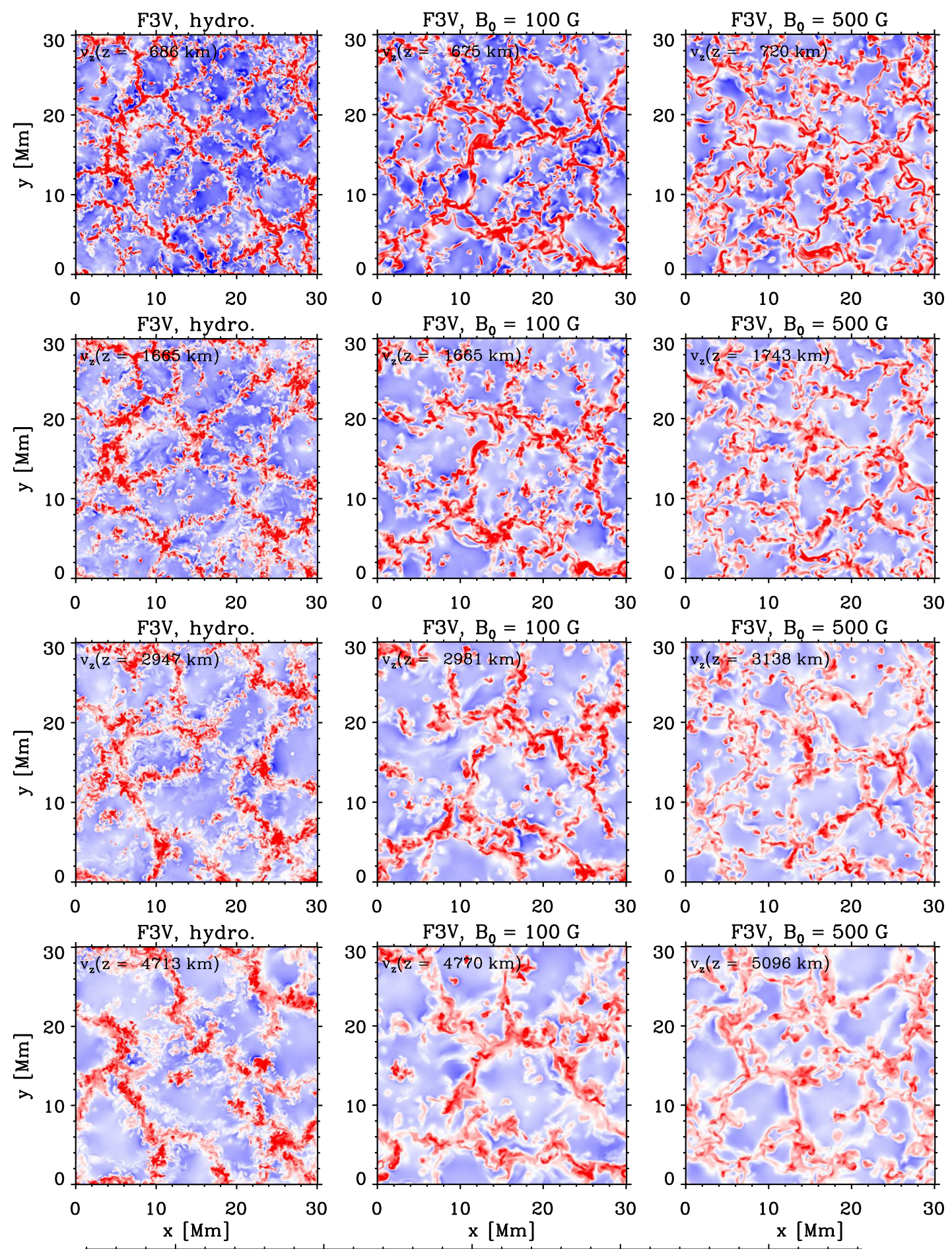

$-10$

$-5$

5

$\mathrm{v}_{\mathbf{z}}\left[\mathrm{km} \mathrm{s}^{-1}\right]$

Figure B.7: Same as Figs. 3.32 and 3.33, but for the F3V simulation. 

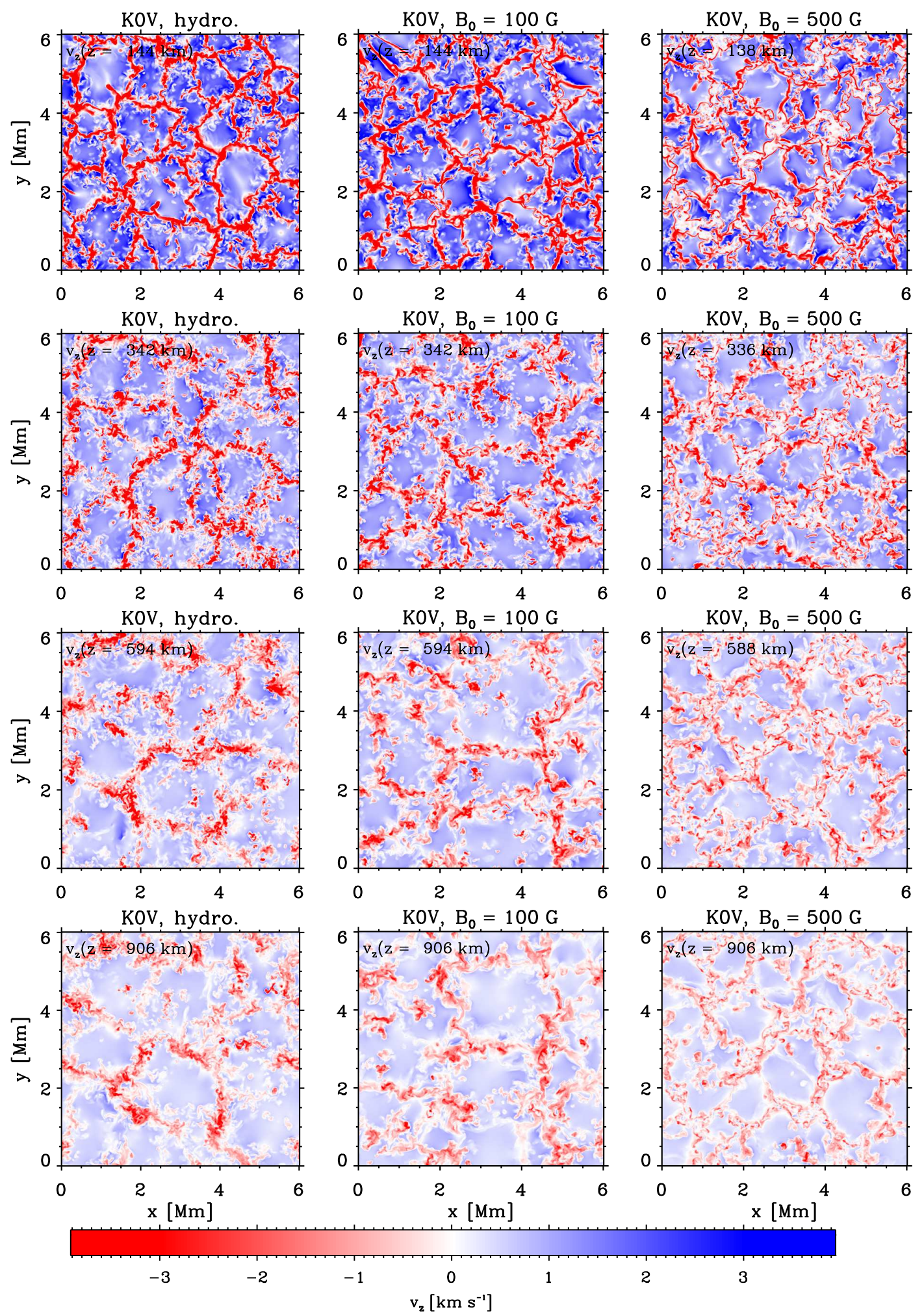

Figure B.8: Same as Figs. 3.32 and 3.33, but for the K0V simulation. 

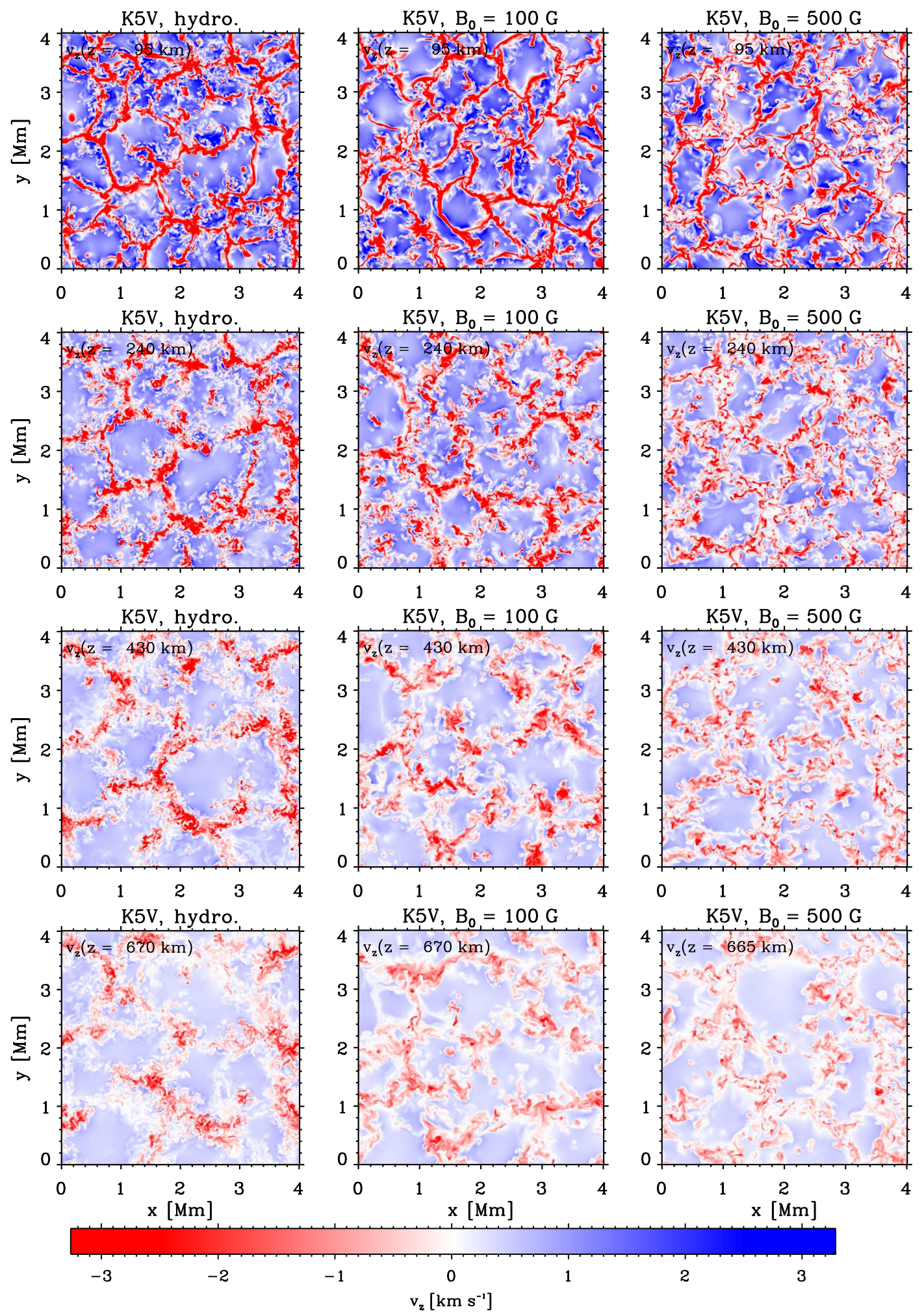

Figure B.9: Same as Figs. 3.32 and 3.33, but for the K5V simulation. 

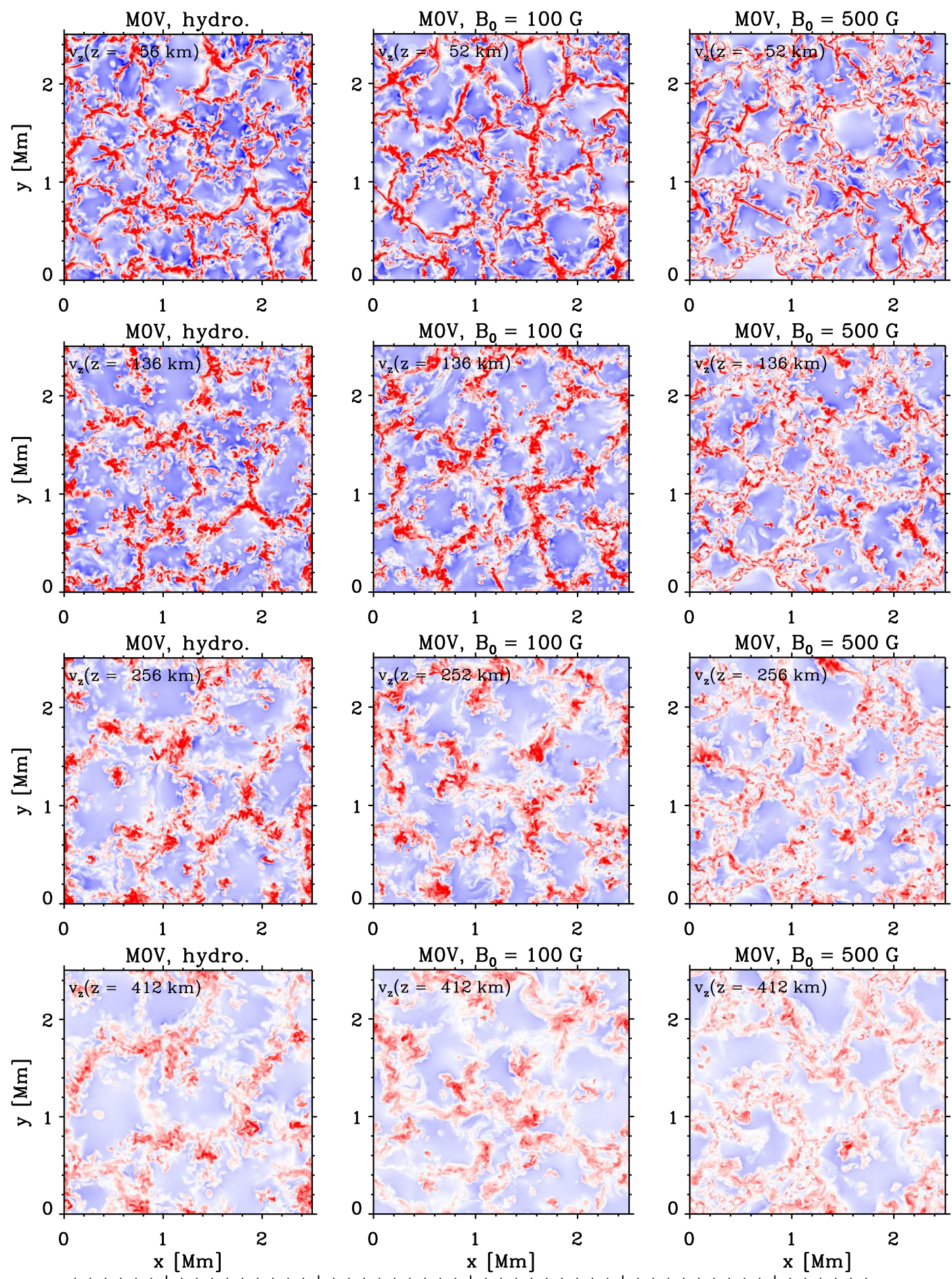

$-2$

$-1$ 0
$\mathbf{z}$$\left[\mathrm{km} \mathrm{s}^{-1}\right]$

Figure B.10: Same as Figs. 3.32 and 3.33, but for the M0V simulation. 

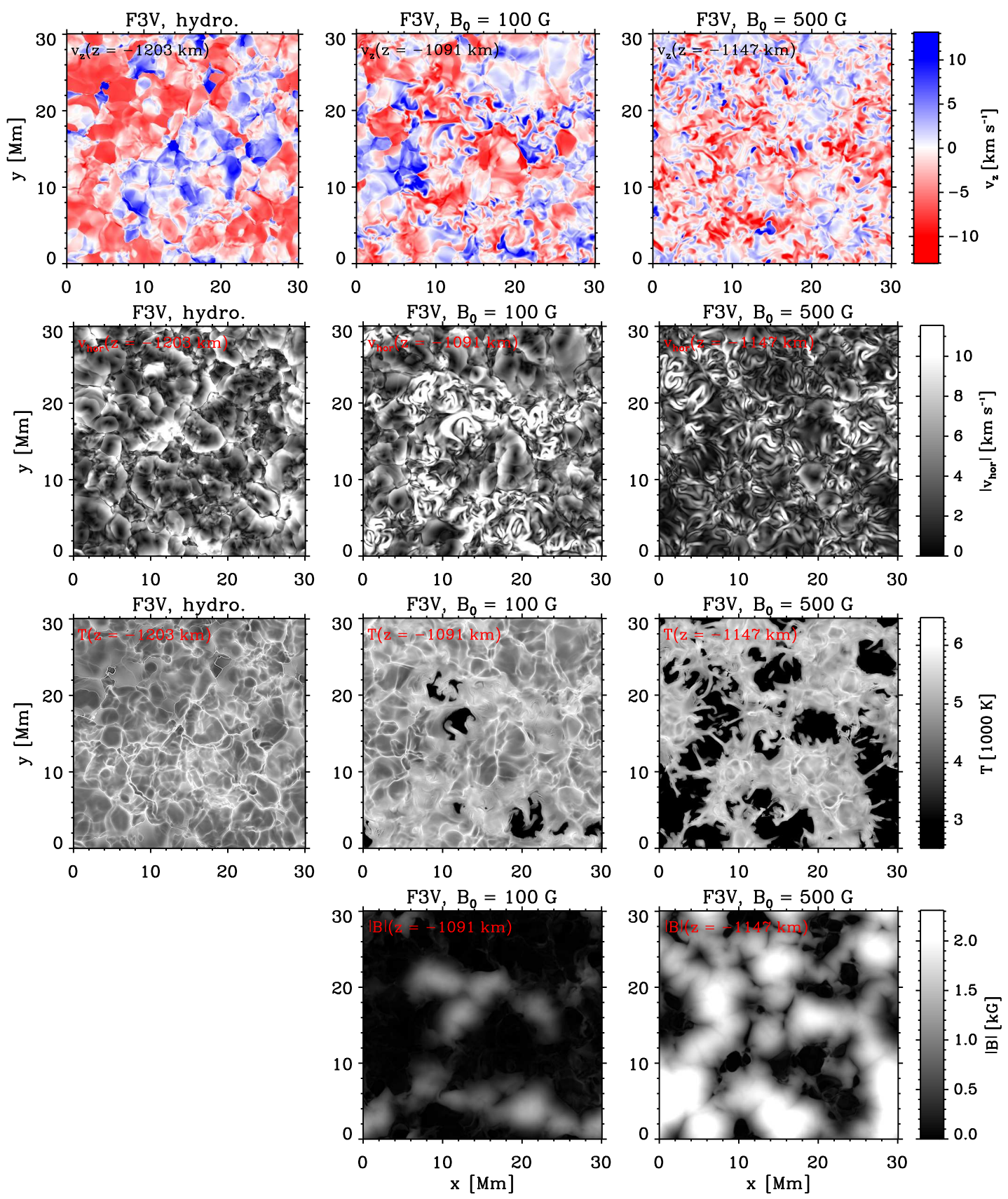

Figure B.11: Same as Figs. 3.34 and 3.35, but for the F3V star. 

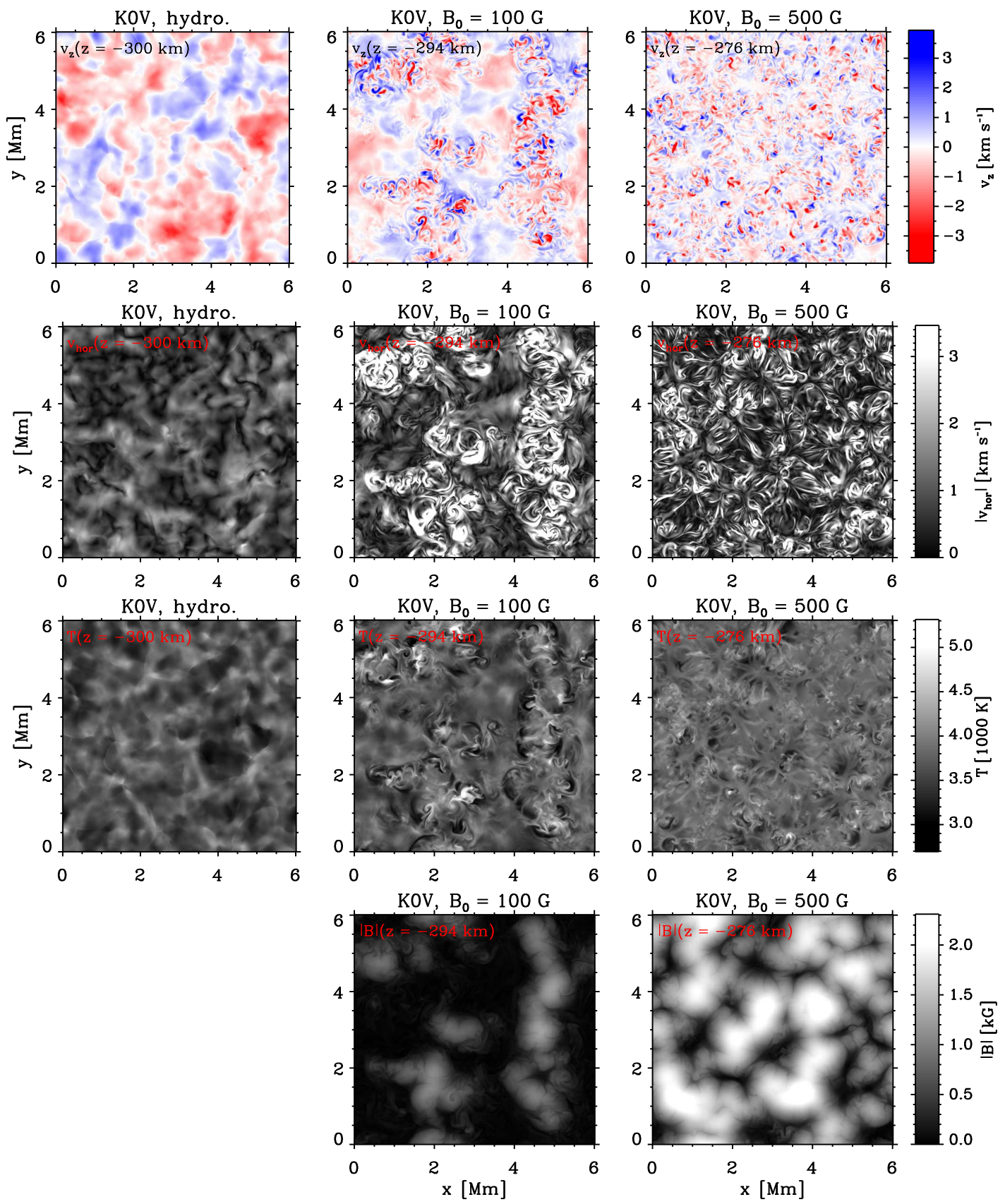

Figure B.12: Same as Figs. 3.34 and 3.35, but for the K0V star. 

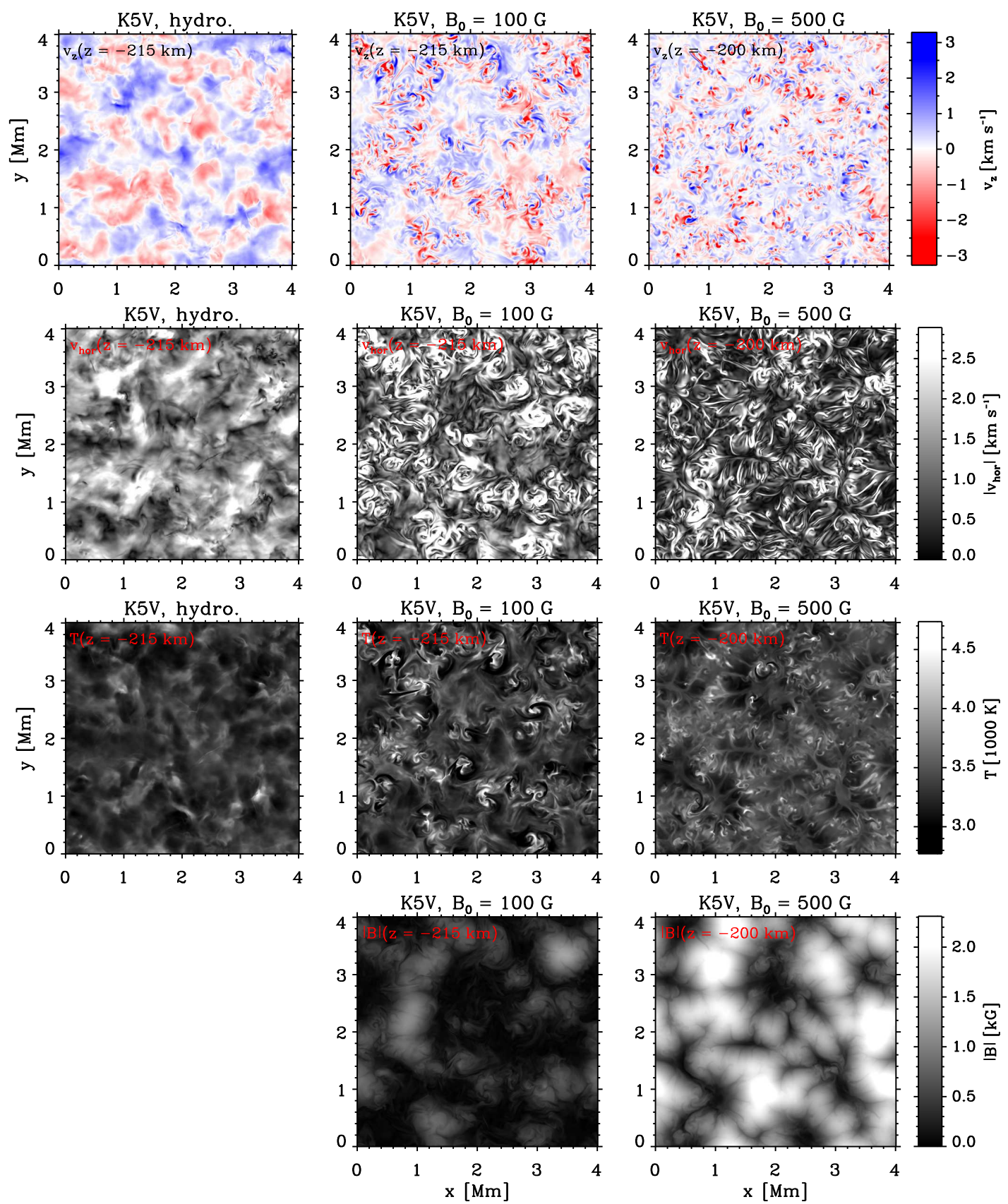

Figure B.13: Same as Figs. 3.34 and 3.35, but for the K5V star. 

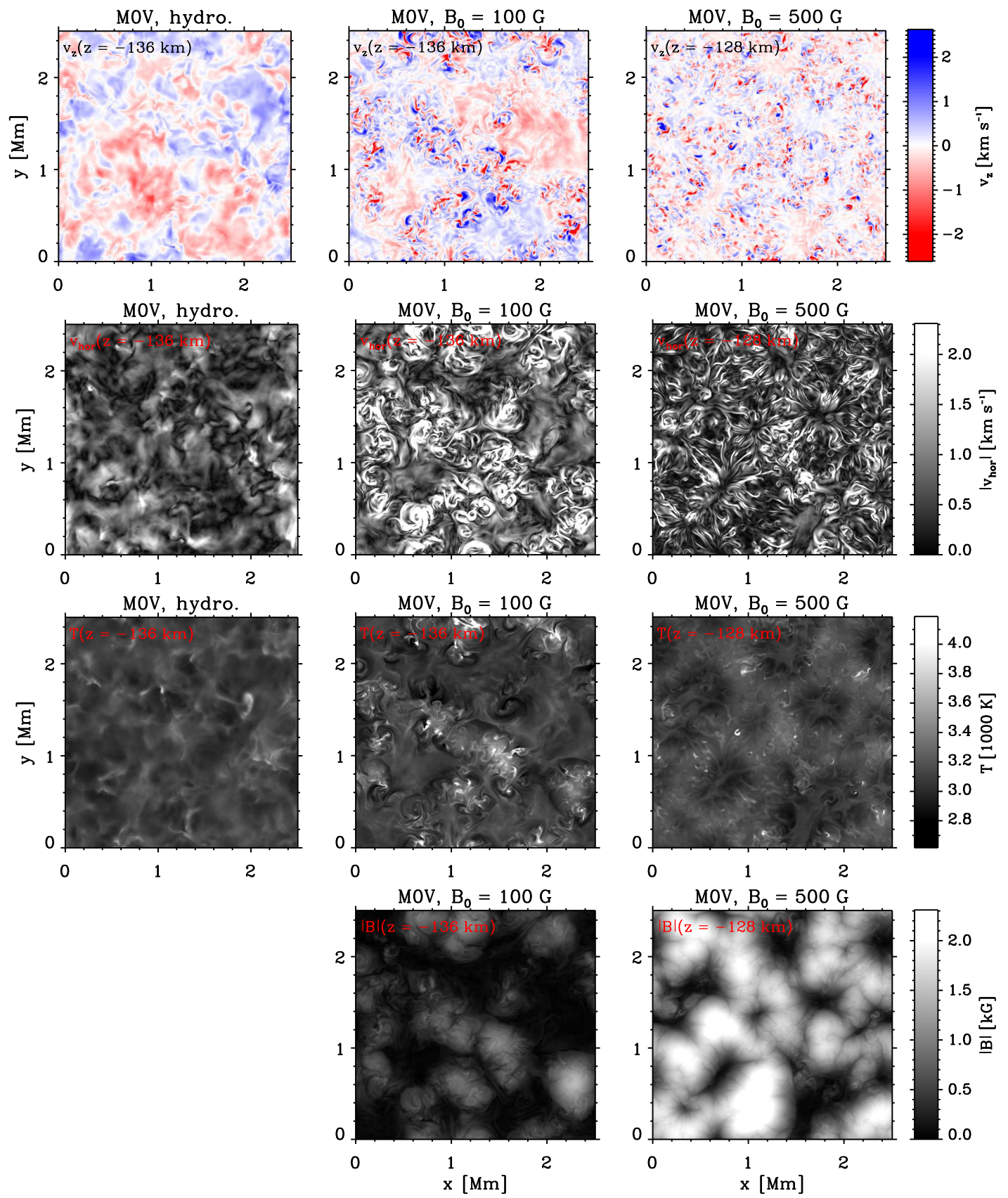

Figure B.14: Same as Figs. 3.34 and 3.35, but for the M0V star. 


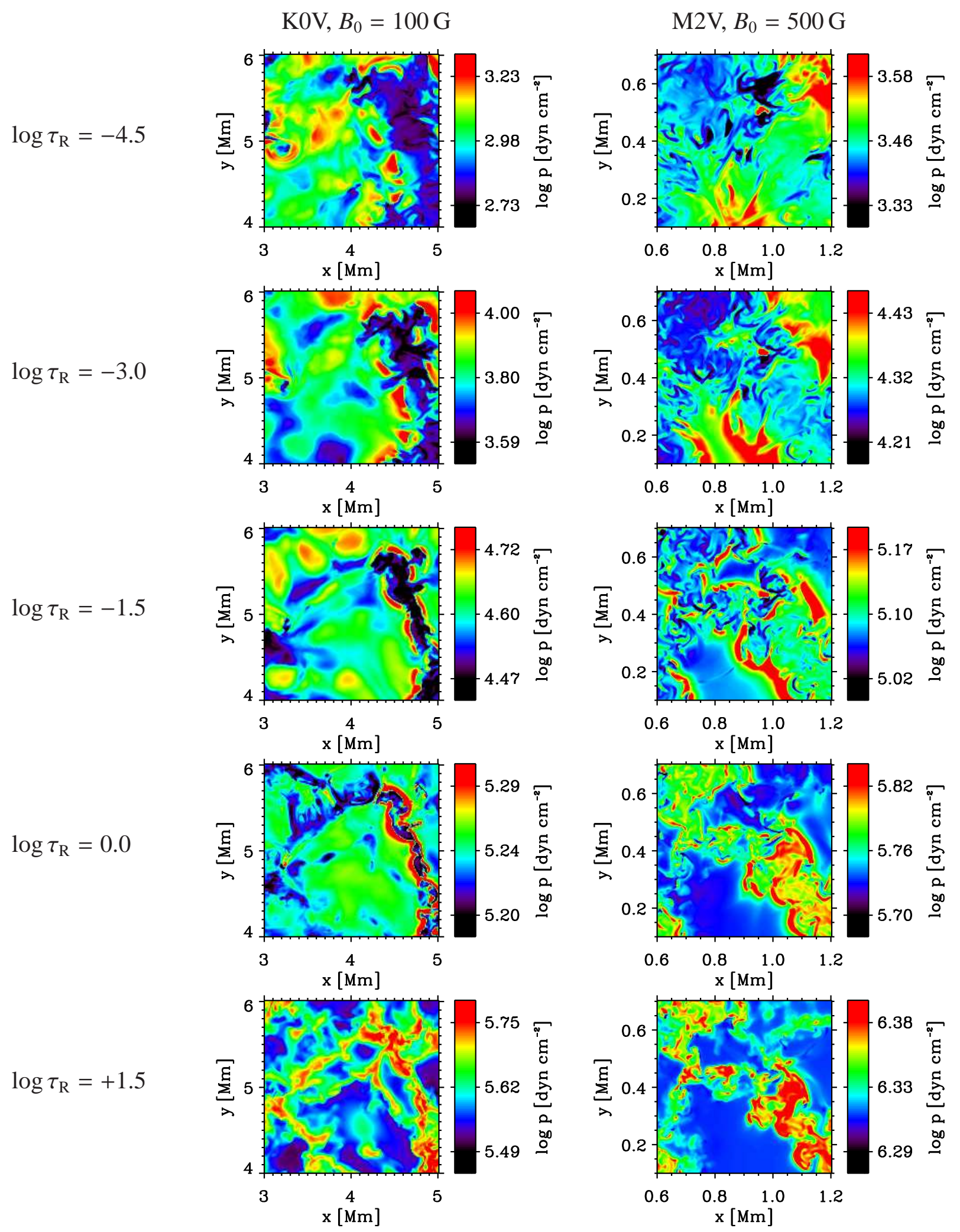

Figure B.15: Maps of the local gas pressure on iso- $\tau_{\mathrm{R}}$ surfaces of a region from the K0V simulation with $B_{0}=100 \mathrm{G}$ and one from the M2V simulation with $B_{0}=500 \mathrm{G}$ (cf. Fig. 3.40). 


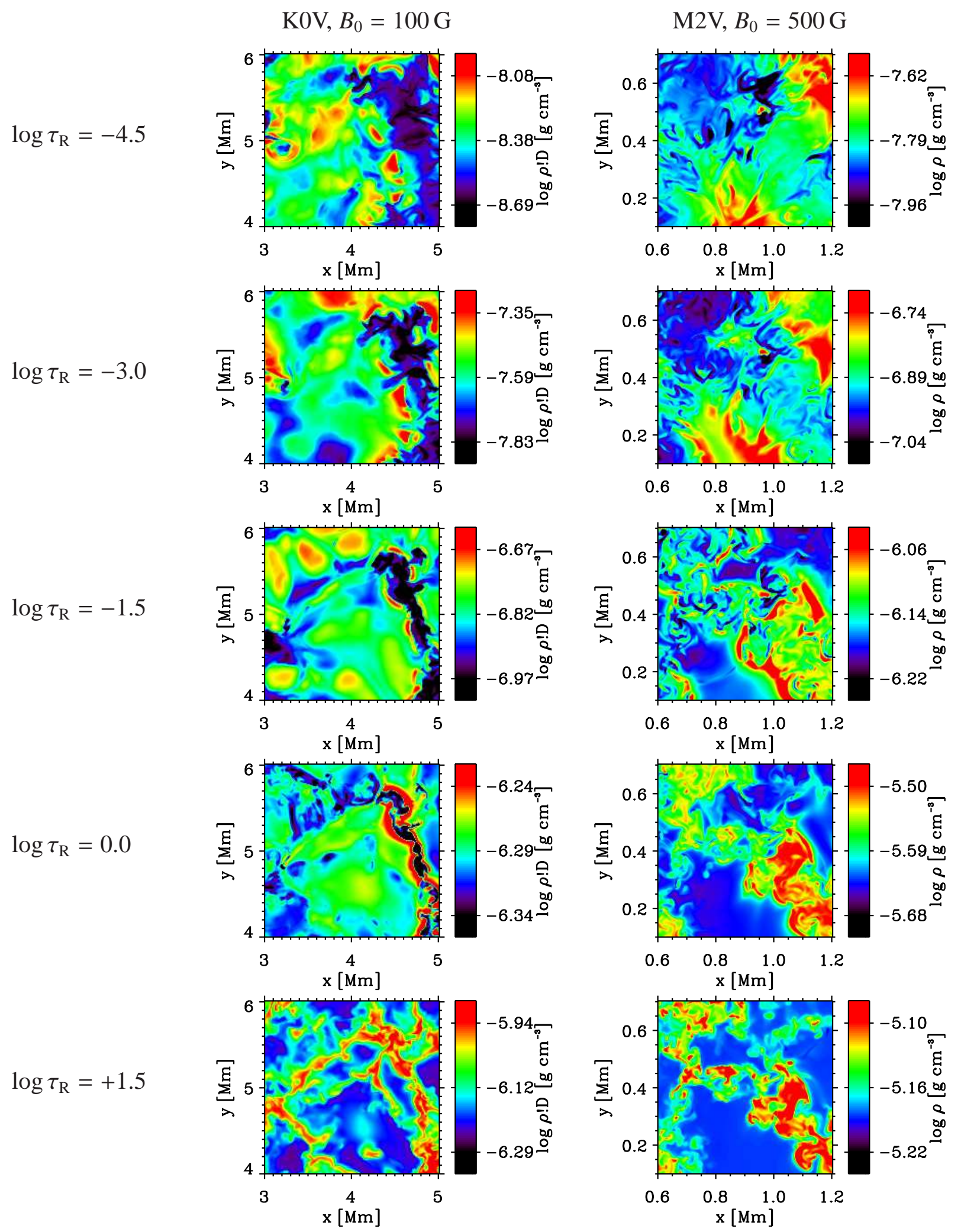

Figure B.16: Maps of the local density on iso- $\tau_{R}$ surfaces of a region from the K0V simulation with $B_{0}=100 \mathrm{G}$ and one from the M2V simulation with $B_{0}=500 \mathrm{G}$ (cf. Figs. 3.40 and B.15). 
$\log \tau_{\mathrm{R}}=-4.5$
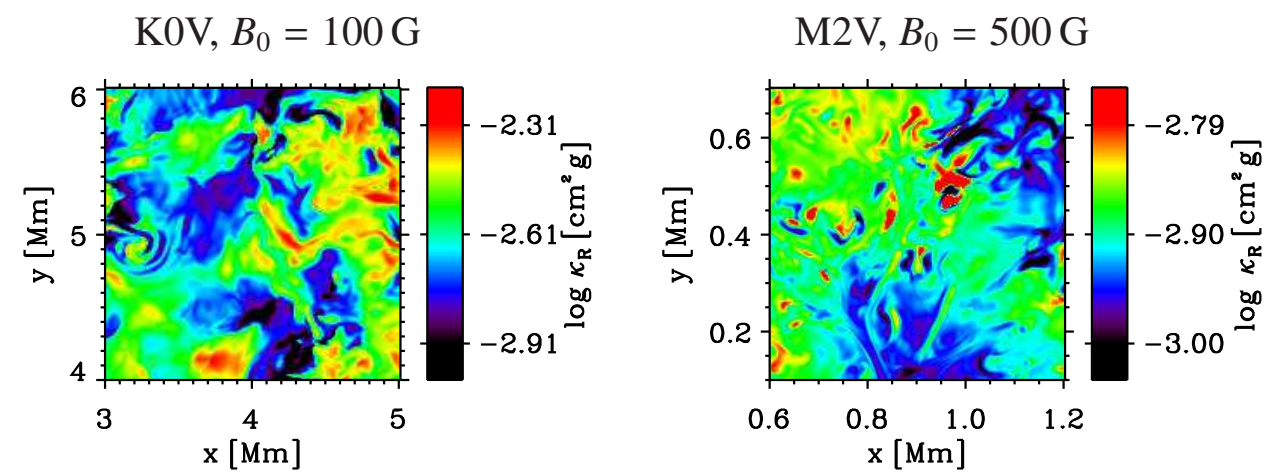

$\log \tau_{\mathrm{R}}=-3.0$
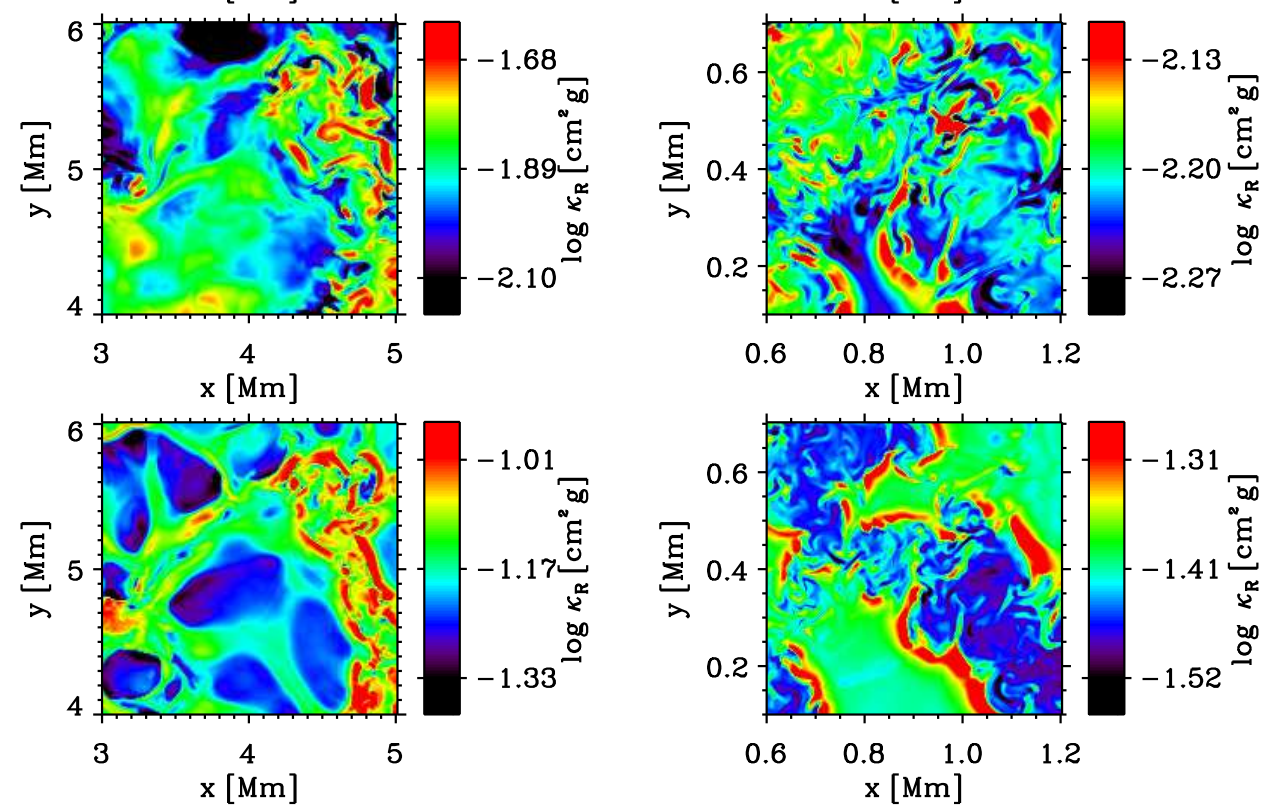

$\log \tau_{\mathrm{R}}=-1.5$
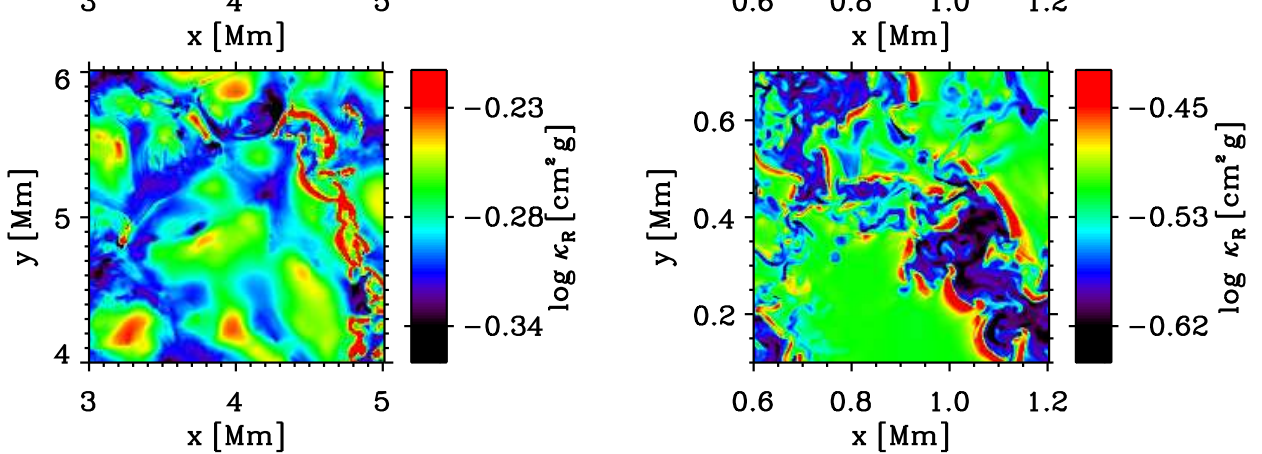

$\log \tau_{\mathrm{R}}=0.0$

$\log \tau_{\mathrm{R}}=+1.5$
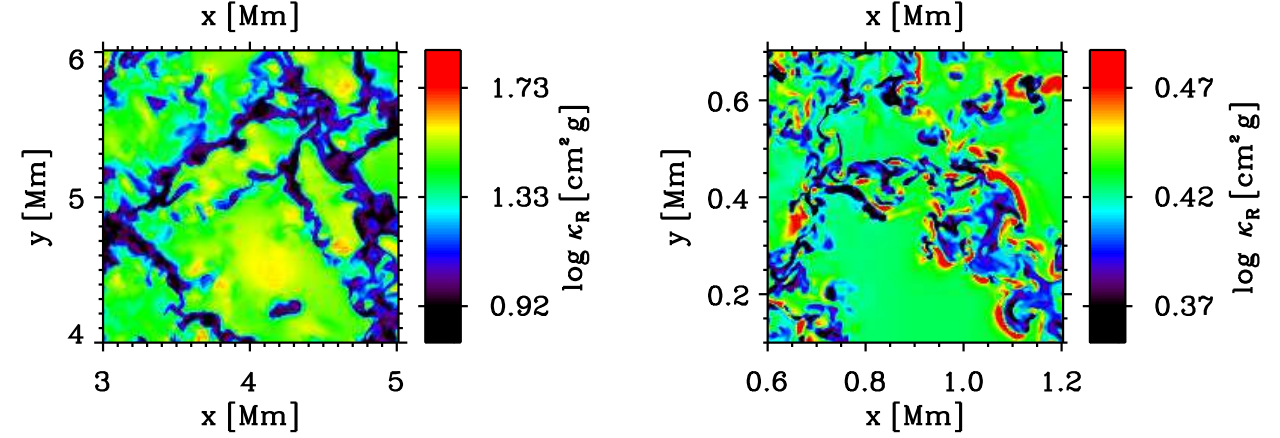

Figure B.17: Maps of the local Rosseland opacity on iso- $\tau_{\mathrm{R}}$ surfaces of a region from the K0V simulation with $B_{0}=100 \mathrm{G}$ and one from the M2V simulation with $B_{0}=500 \mathrm{G}$ (cf. Figs. 3.40, B.15, and B.16). 

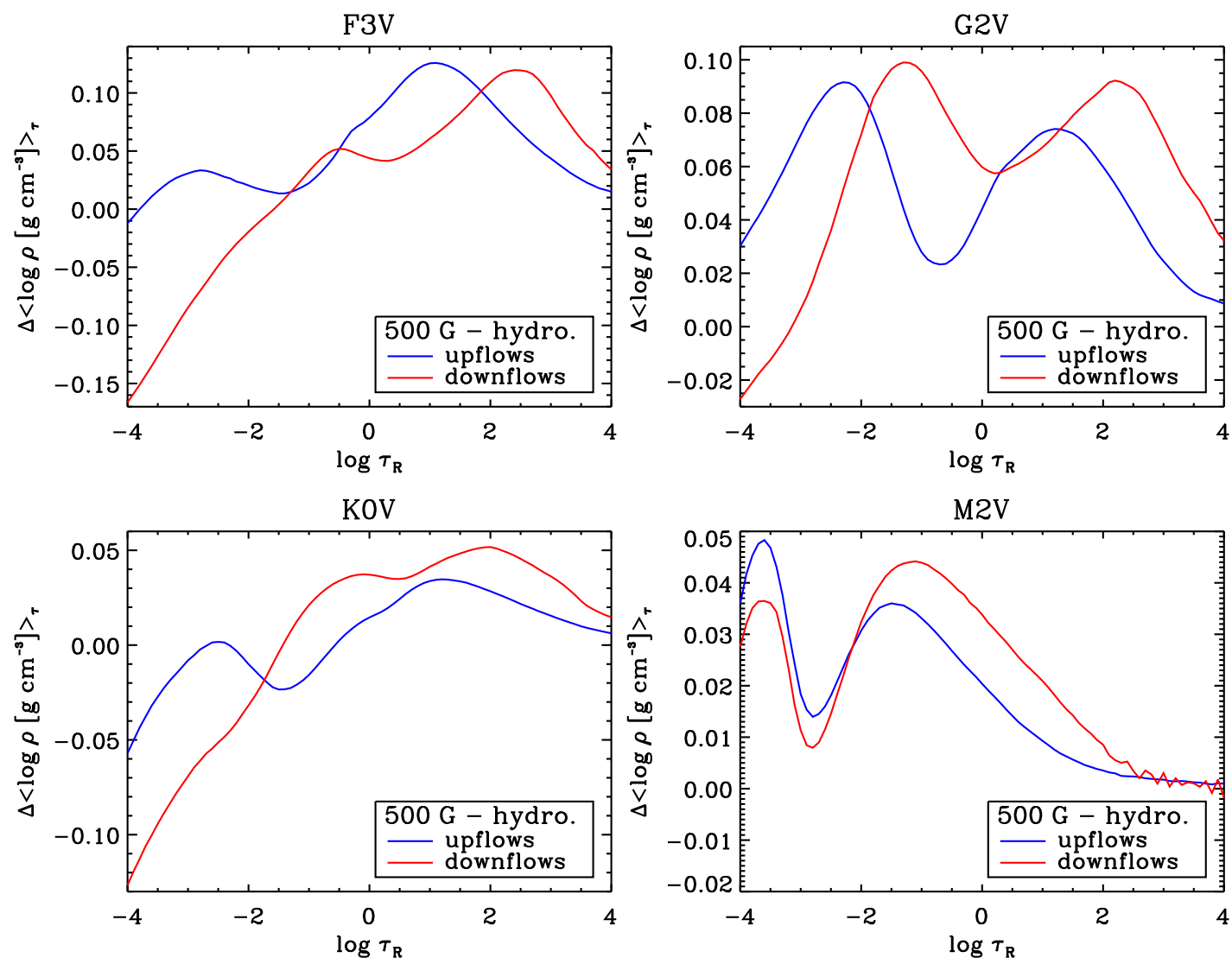

Figure B.18: Average density difference between the $500 \mathrm{G}$ and non-magnetic runs in upand downflows of the F3V, G2V, K0V, and M2V simulations. 

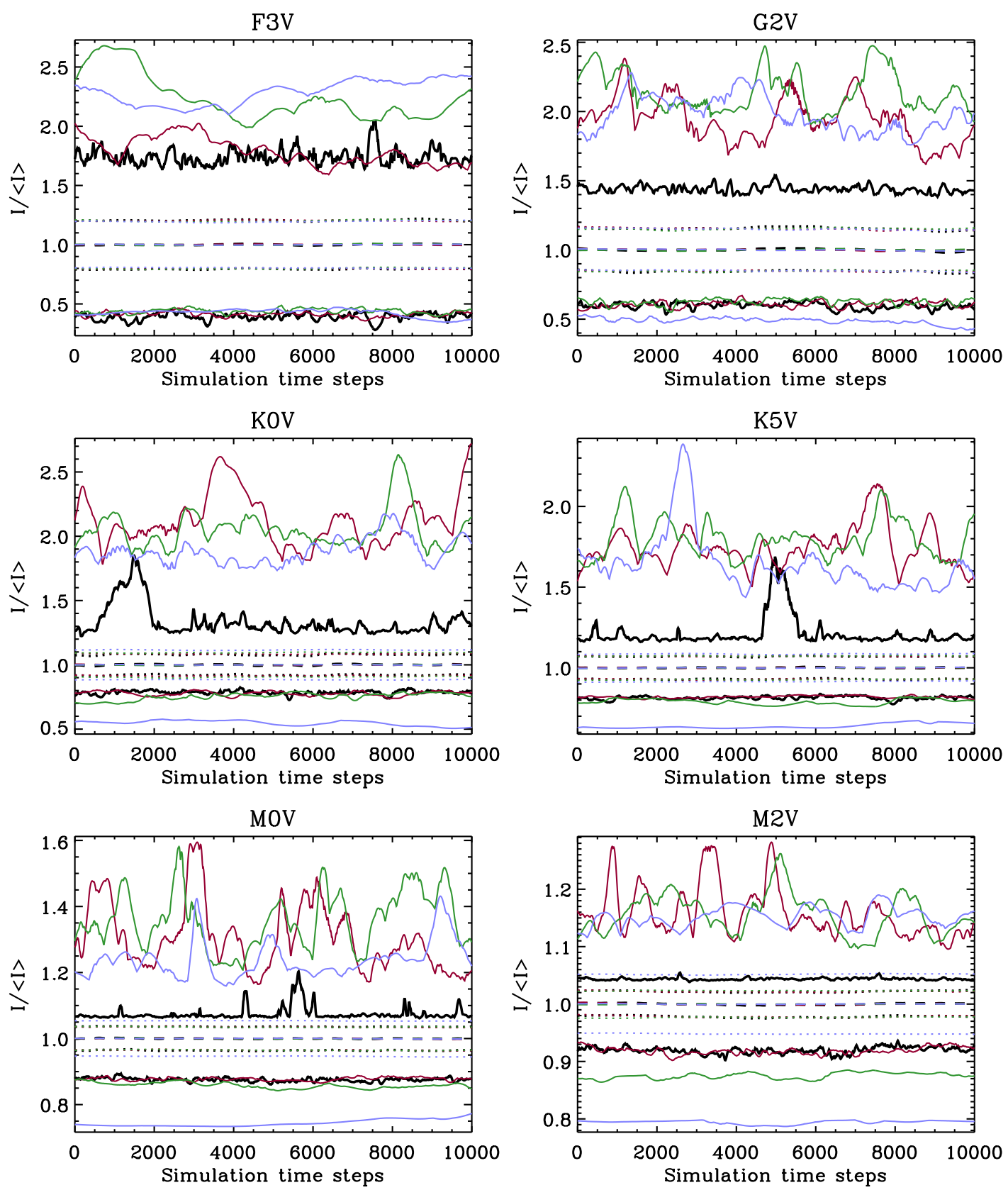

Figure B.19: Extreme values (solid), standard deviations (dotted), and mean value (dashed) of the intensity of all simulation runs over the 10000 simulation time steps which were the basis for most of the analysis in this thesis. All intensities are normalised to the mean intensity of the respective simulation. The colour code is the same as in Fig. 3.24: black $=$ non-magnetic run, dark red $=20 \mathrm{G}$ run, green $=100 \mathrm{G}$ run, light violet $=500 \mathrm{G}$. The "spikes" in the maximum value of the non-magnetic K0V, K5V, and M0V simulations are caused by strong vertical vortices (see Sect. 4.2.2). 

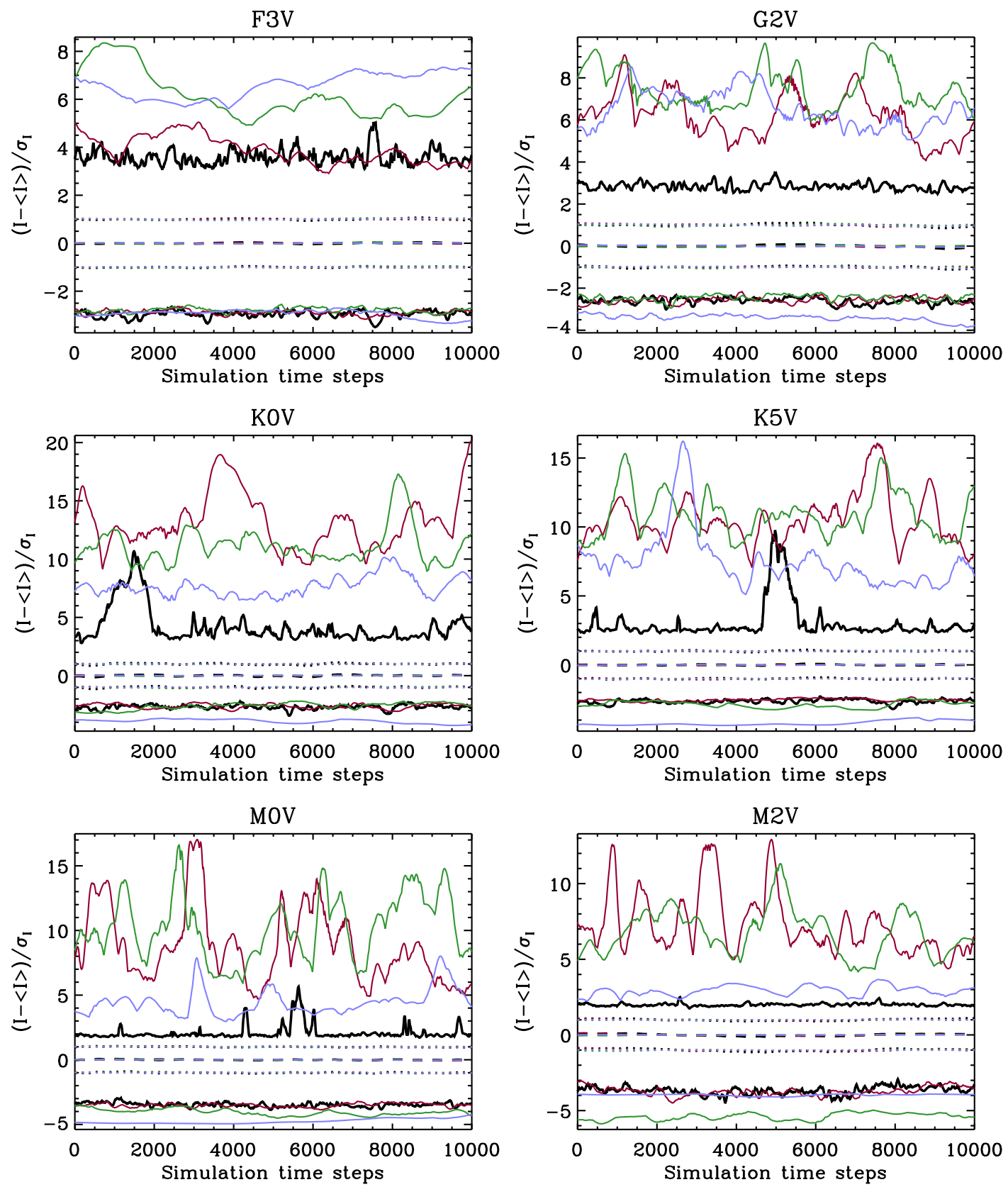

Figure B.20: Same as Fig. B.19 but in normalised intensity fluctuations $\hat{I}$ of Eq. (4.1). Note that since the standard deviation depends on $B_{0}$ especially in the $\mathrm{M}$ stars, the dark regions on the M2V star have a higher $\hat{I}$ value in the $500 \mathrm{G}$ run than in the $100 \mathrm{G}$ run, although they are actually darker (cf. Fig. B.19). 

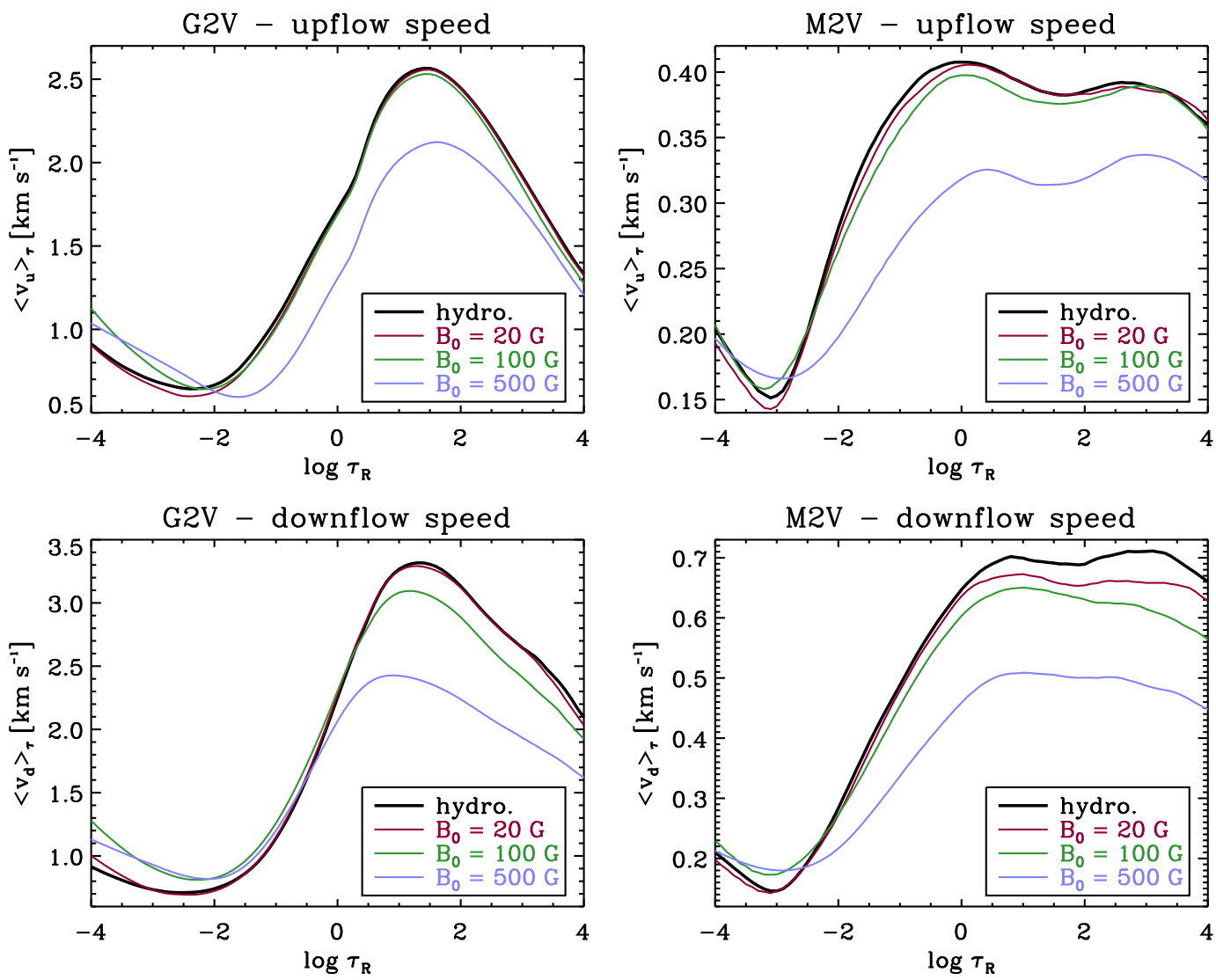

Figure B.21: Convective up- and downflow speeds in the G2V and M2V simulations averaged on surfaces of constant optical depth. 

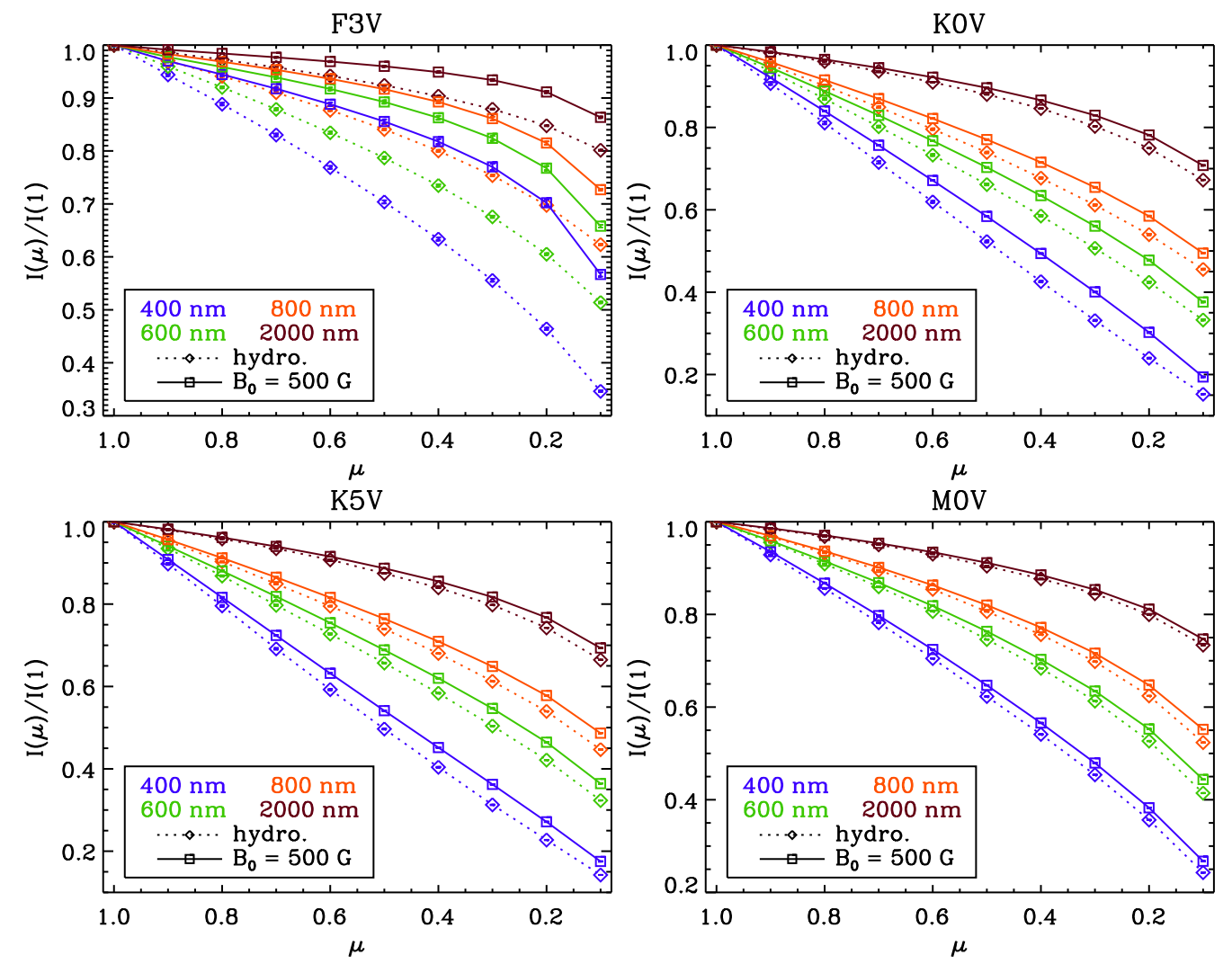

Figure B.22: Same as Fig. 5.5, but for the F3V, K0V, K5V, and M0V simulations. 
B Additional figures
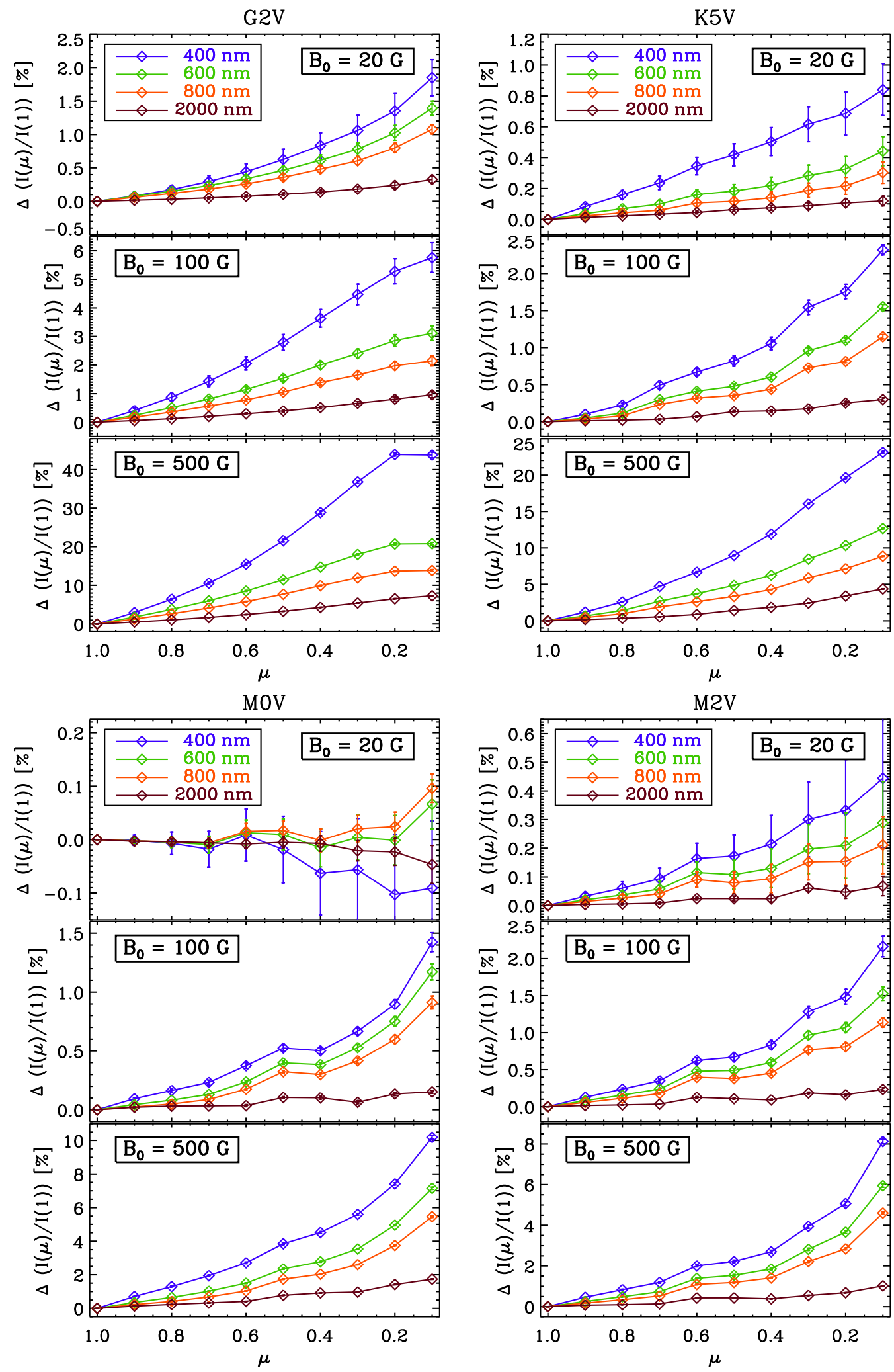

Figure B.23: Same as Fig. 5.6 but for the G2V, K5V, M0V, and M2V simulations. 

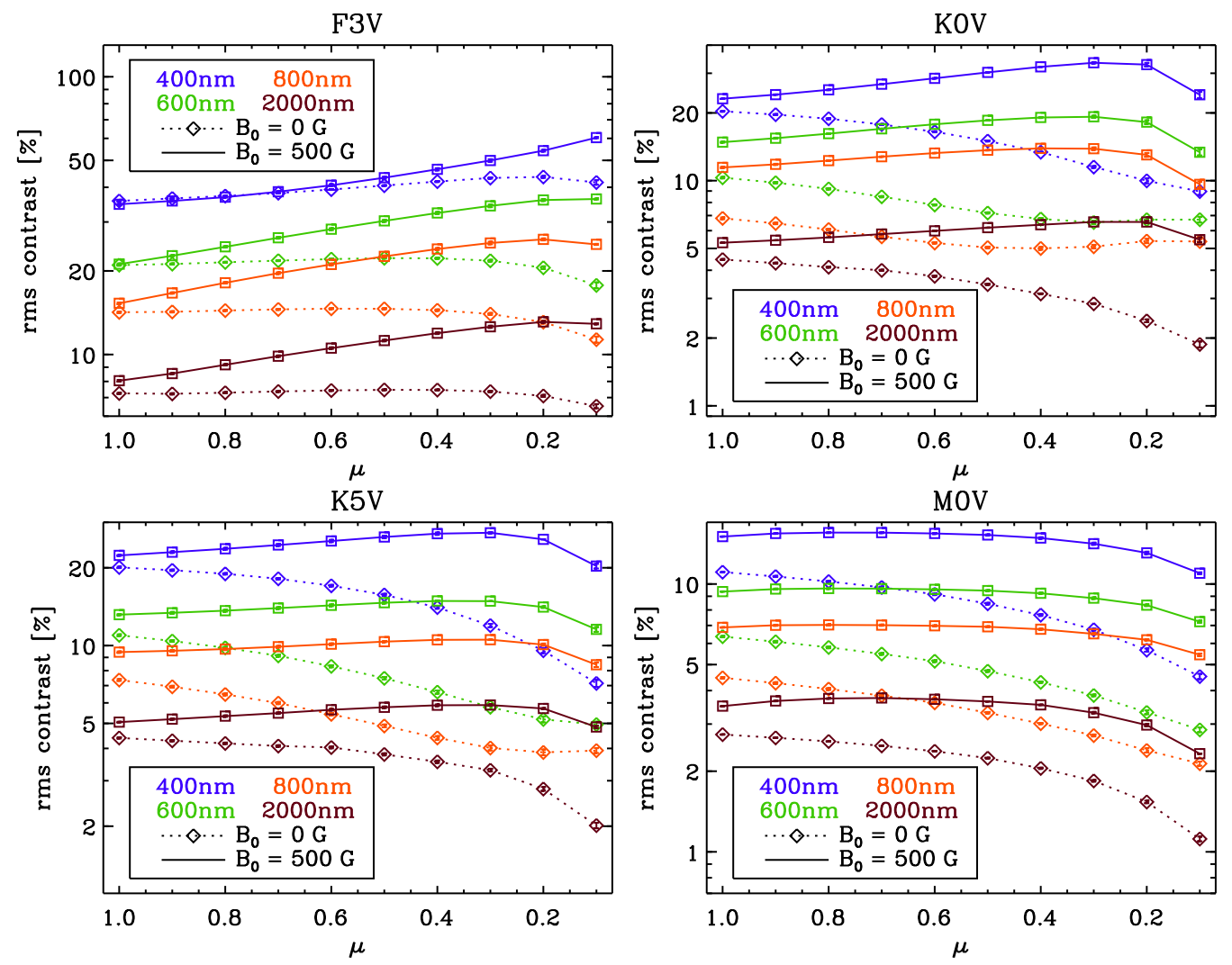

Figure B.24: Same as Fig. 5.7, but for the F3V, G2V, K5V, and M0V simulations. 
F3V
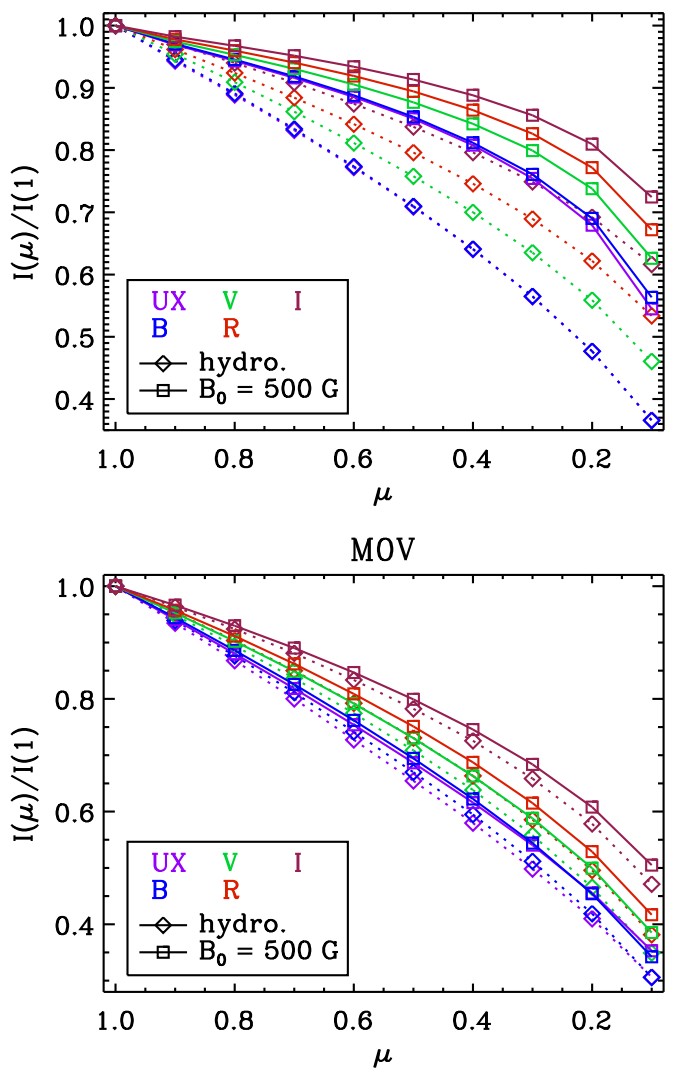

F3V

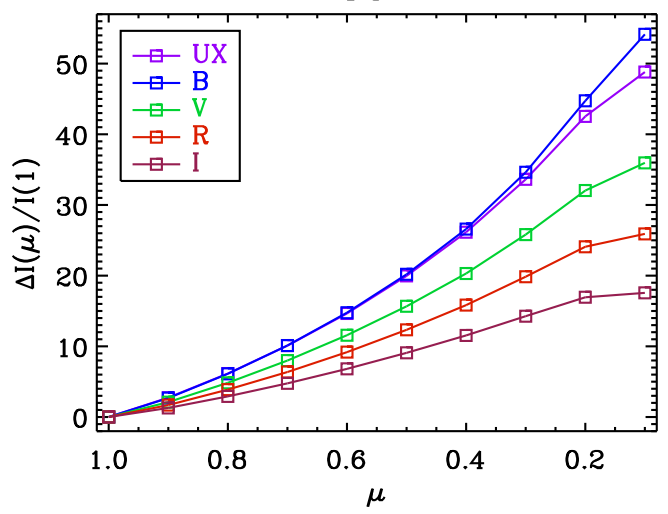

MOV

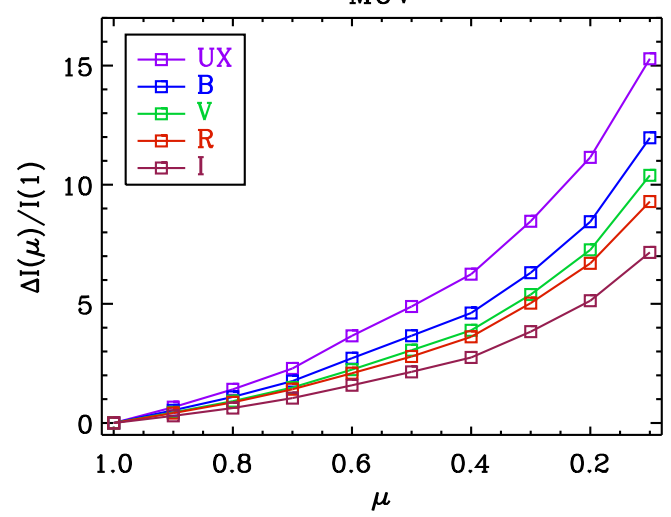

Figure B.25: Same as Fig. 5.8 but for the F3V and M0V star. 

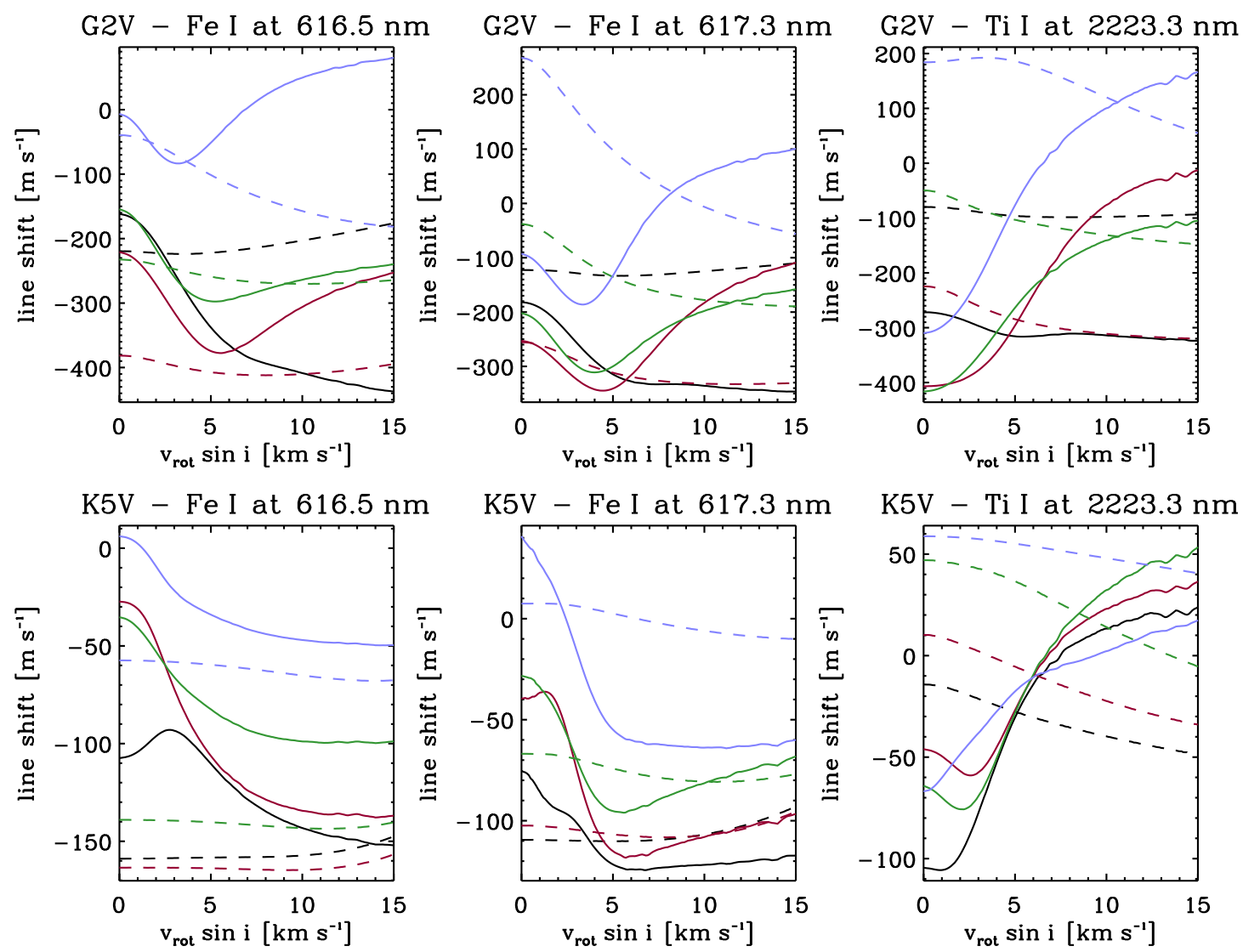

$\mathrm{m} 5 \mathrm{~V}-\mathrm{Ti} \mathrm{I}$ at $2223.3 \mathrm{~nm}$
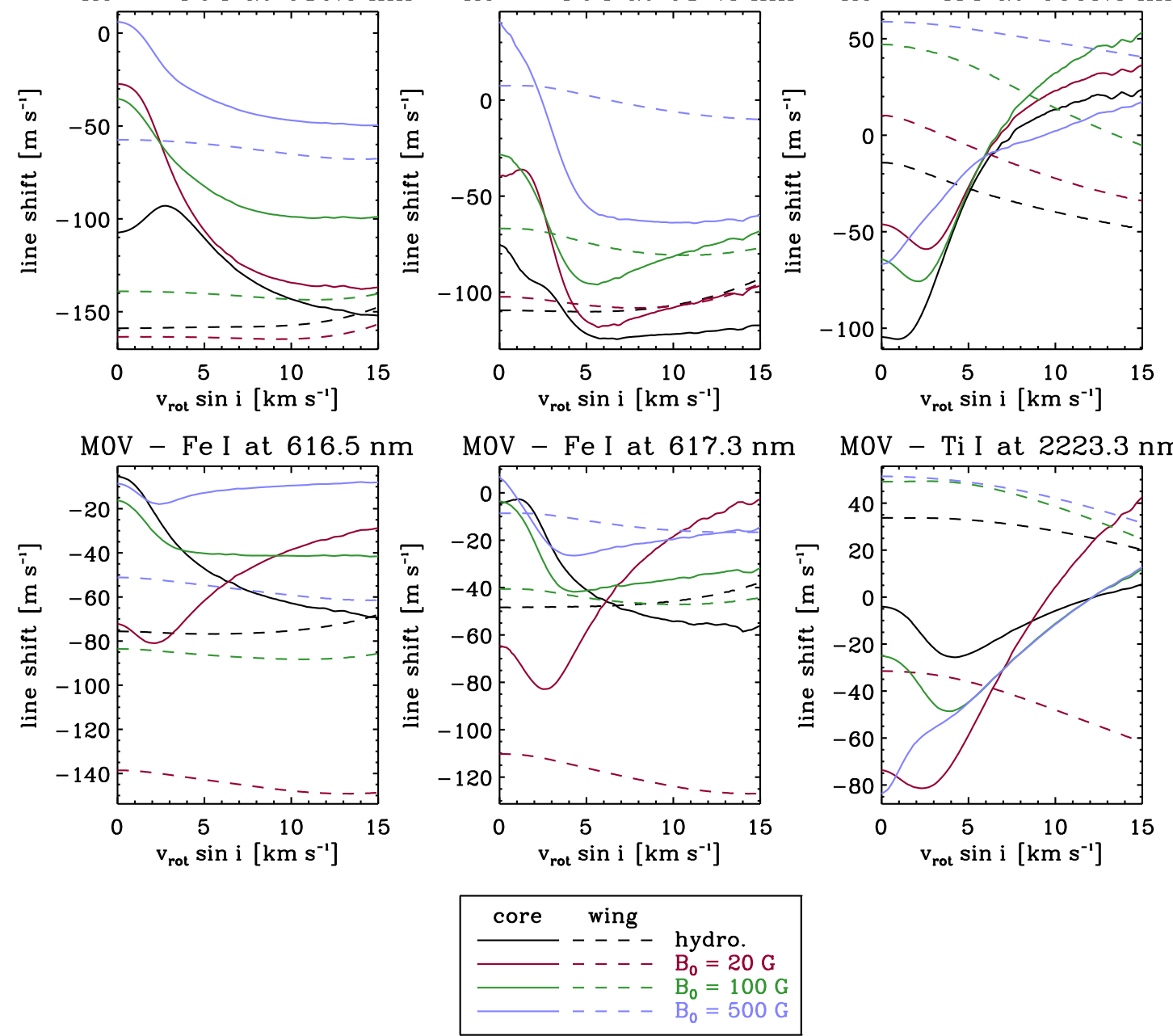

Figure B.26: Same as Fig. 6.22 but for the G2V, K5V, and M0V simulations. 

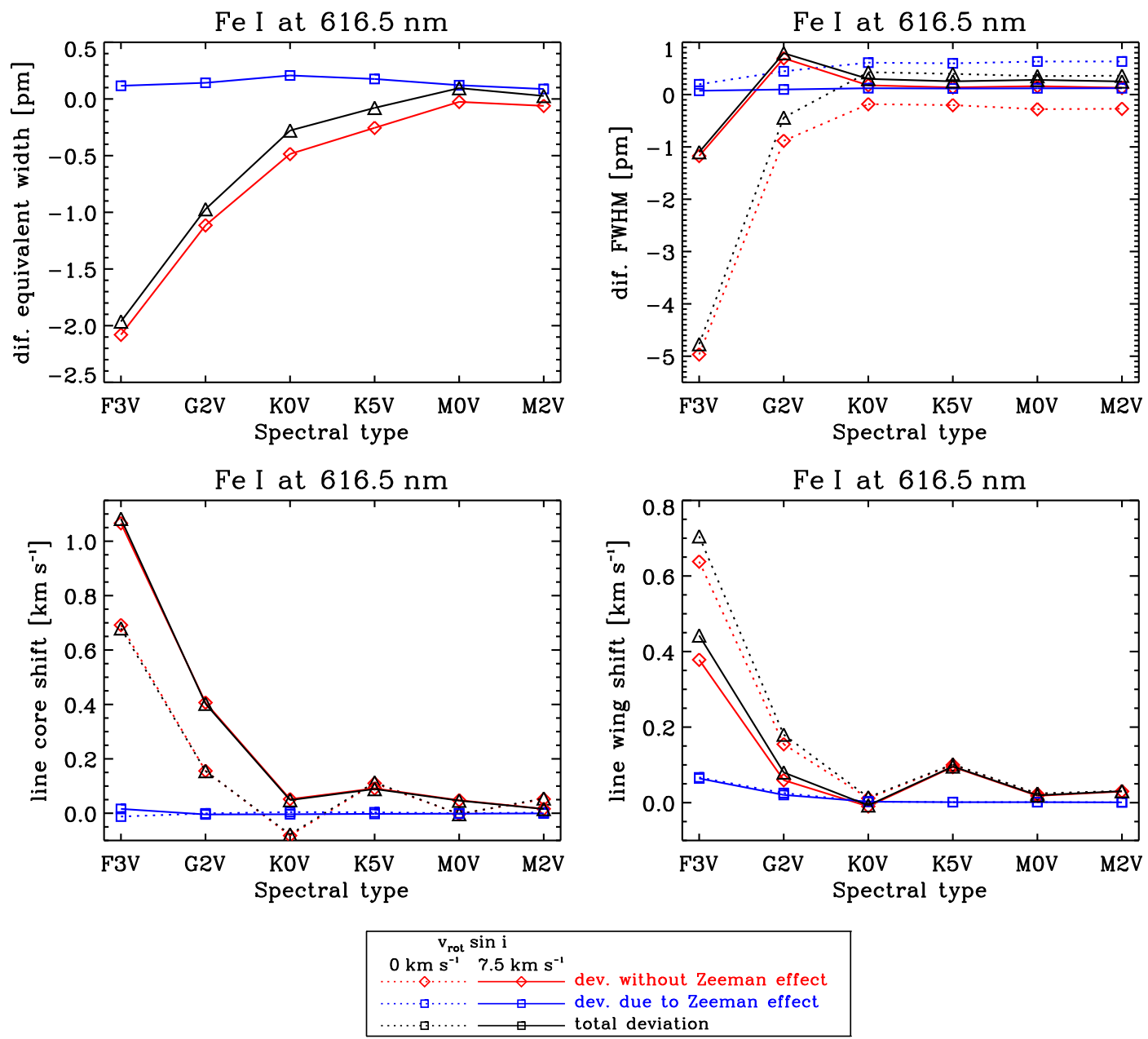

Figure B.27: Same as Fig. 6.25 but for the Fe I line at $616.5 \mathrm{~nm}$. 

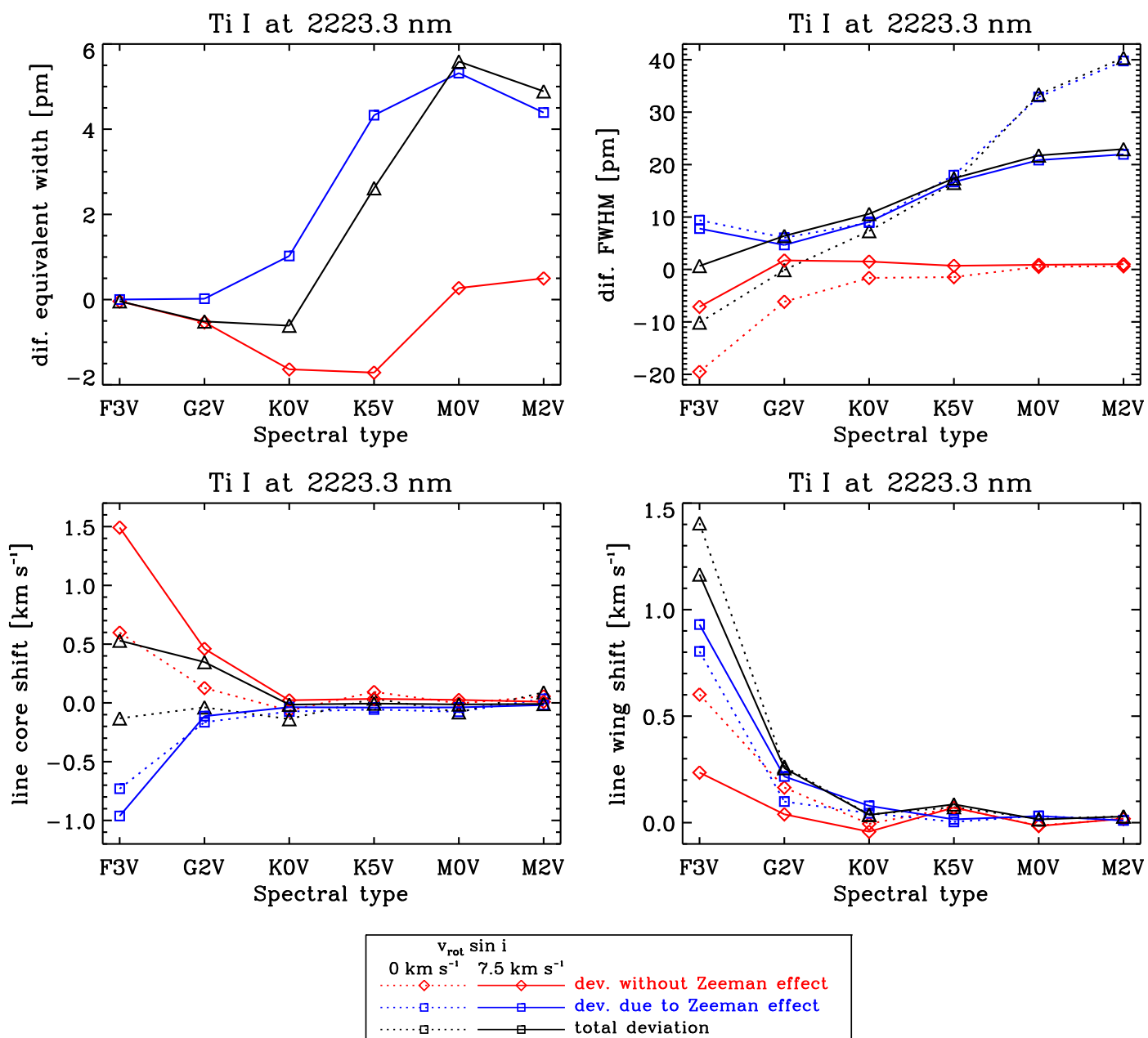

Figure B.28: Same as Figs. 6.25 and B.27 but for the Ti I line at $2223.3 \mathrm{~nm}$. 


\section{Publications}

\section{Refereed publications}

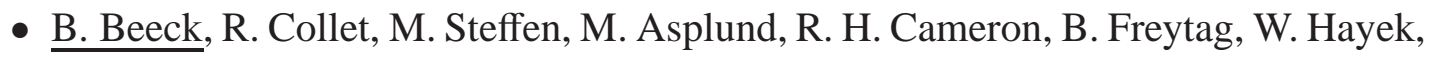
H.-G. Ludwig, and M. Schüssler (2012). Simulations of the solar near-surface layers with the CO5BOLD, MURaM, and Stagger codes. Astronomy $\mathcal{E}$ Astrophysics 539, A 121

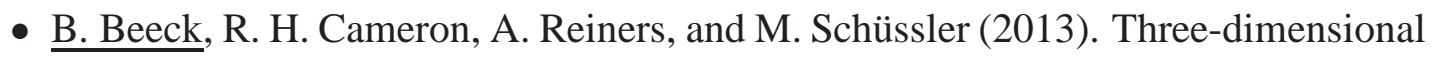
simulations of near-surface convection in main-sequence stars. I. Overall structure. Astronomy $\mathcal{G}$ Astrophysics 558, A48

- B. Beeck, R. H. Cameron, A. Reiners, and M. Schüssler (2013). Three-dimensional simulations of near-surface convection in main-sequence stars. II. Properties of granulation and spectral lines. Astronomy $\mathcal{E}$ Astrophysics 558, A49

- L. Bharti, B. Beeck, and M. Schüssler (2010). Properties of simulated sunspot umbral dots. Astronomy \& Astrophysics 510, A12

- S. Danilovic, B. Beeck, A. Pietarila, M. Schüssler, M., S. K. Solanki, V. Martínez Pillet, J. A. Bonet, J. C. del Toro Iniesta, V. Domingo, P. Barthol, T. Berkefeld, A. Gandorfer, M. KnÃúlker, W. Schmidt, A. M. Title (2010). Transverse Component of the Magnetic Field in the Solar Photosphere Observed by SUNRISE. The Astrophysical Journal 723, L149-L153

\section{Conference proceedings}

- B. Beeck, S. Schuh, T. Nagel, and I. Traulsen (2009). Towards a dynamical mass of a PG 1159 star: radial velocities and spectral analysis of SDSS J212531-010745. JENAM 2008 Symposium No 4: Asteroseismology and Stellar Evolution, Communications in Asteroseismology 159, 111-113

- B. Beeck, M. Schüssler, and A. Reiners (2011). MHD Simulations Reveal Crucial Differences Between Solar and Very Cool Star Magnetic Structures. $16^{\text {th }} \mathrm{Cam}^{-}$ bridge Workshop on Cool Stars, Stellar Systems, and the Sun. ASP Conference Series 448, 1071 
- Ch. Helling, E. Pedretti, S. Berdyugina, A. A. Vidotto, B. Beeck, E. Baron, A. P. Showman, E. Agol, and D. Homeier (2011). Aspects of Multi-Dimensional Modelling of Substellar Atmospheres. $16^{\text {th }}$ Cambridge Workshop on Cool Stars, Stellar Systems, and the Sun. ASP Conference Series 448, 403

- J. Morin, M. Jardine, A. Reiners, D. Shulyak, B. Beeck, G. Hallinan, L. Hebb, G. Hussain, S. V. Jeffers, O. Kochukhov, A. Vidotto, and L. Walkowicz (2013). Multiple views of magnetism in cool stars. 17 th $^{\text {th }}$ ambridge Workshop on Cool Stars, Stellar Systems, and the Sun. Astronomische Nachrichten 334, 48-52 


\section{Acknowledgements}

First and foremost, I want to thank my supervisors, Ansgar Reiners and Manfred Schüssler. My PhD project largely benefited from their deep knowledge in two complementary fields of research as well as from their encouragement and advice. Ansgar and Manfred were both always available to solve scientific as well as personal issues. I learned a lot about scientific writing from Manfred through his thorough revision of our paper drafts and of the thesis, which is much appreciated.

Many thanks to Robert F. Stein, who volunteered to review my thesis as a third referee. As PhD student of the International Max Planck Research School (IMPRS) on Physical Processes in the Solar System and Beyond at the Universities of Braunschweig and Göttingen, I owe the former coordinator, Dieter Schmitt, many thanks. Sadly, Dieter died shortly before I submitted my thesis, but I am sure his memory as a very friendly and engaged coordinator of the research school will live on.

During the past months, the new coordinator of the IMPRS, Sonja Schuh, helped me through the more bureaucratic steps towards the submission of the thesis, for which I am very grateful.

I thank the members of both working groups (from the MPS and the IAG) for interesting science discussions and a nice working environment. In particular, I want to thank Robert Cameron, who was like an unofficial third advisor to me and who I could bother with small technical and scientific questions of all kinds.

I acknowledge research funding by the Deutsche Forschungsgemeinschaft (DFG) under the grant $S F B$ 963/1, project A16. I acknowledge financial support by the International Max Planck Research School (IMPRS) on Physical Processes in the Solar System and Beyond at the Universities of Braunschweig and Göttingen.

I would like to thank my friends Philip and Meike for cheering me up during lunch breaks, whenever the work did not go as smoothly as I would have liked. I also thank my friends from the choir Unicante for the nice and always welcome distraction from my work! Unicante has been an important part of my life in Göttingen and a source of motivation during the past years.

I thank my family for their financial and moral support. And last but certainly not least, I want to thank my girlfriend, Ines, for her love and support during the past months! 



\title{
Lebenslauf
}

\author{
Name: Benjamin Beeck \\ Geburtstag: 14. Mai 1983 \\ Geburtsort: Eutin \\ Staatsangehörigkeit: deutsch

\section{Schulausbildung} \\ 09/1989 - 07/1993 Grundschule am Kleinen See, Eutin \\ 08/1993 - 06/2002 Johann-Heinrich-Voss Gymnasium, Eutin \\ 08.06.2002 Abitur
}

\section{Zivildienst}

09/2002 - 06/2003 Evangelisch-Lutherische Kirchengemeinde Eutin

\section{Studium (mit Promotion)}

$\begin{aligned} & \text { 10/2003-07/2009 } \begin{array}{l}\text { Physikstudium an der Georg-August-Universität } \\ \text { Göttingen } \\ \text { Diplomvorprüfung }\end{array} \\ & 05 / 2008-05 / 2009 \begin{array}{l}\text { Diplomarbeit ,Determination of the Dynamic } \\ \text { Masses for the Close PG 1159 Binary SDSS } \\ \text { J212531.92-010745.9“ } \\ \text { Diplomprüfung }\end{array} \\ & \text { seit 09/2009 } \begin{array}{l}\text { Promotion im Rahmen der IMPRS (MPI für Sonnen- } \\ \text { systemforschung) an der Universität Göttingen }\end{array} \\ & \text { IMPRS-Stipendiat } \\ & \text { seit 06/2012 } \\ & \text { wissenschaftlicher Mitarbeiter im Rahmen des } \\ & \text { Sonderforschungsbereichs (SFB) 963, Projekt } \\ & \text { A16, am MPI für Sonnensystemforschung in Göt- } \\ & \text { tingen }\end{aligned}$

